\title{
Overcoming the Naphthyl Requirement in Stereospecific Cross-Couplings to Form Quaternary Stereocenters
}

Jianyu Xu, Olivia P. Bercher, Mary P. Watson*

Department of Chemistry and Biochemistry, University of Delaware, Newark, DE 19716

\section{Supplementary Information}

General Information S2

Optimization Studies S3

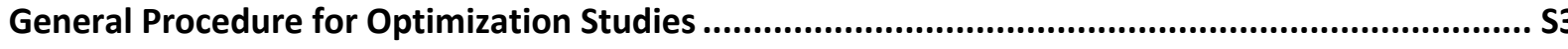

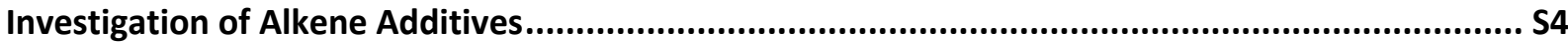

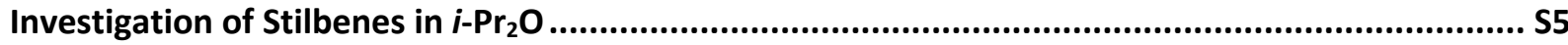

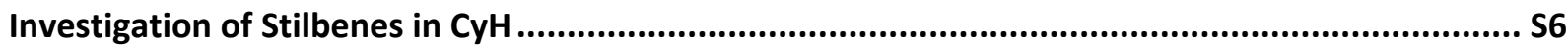

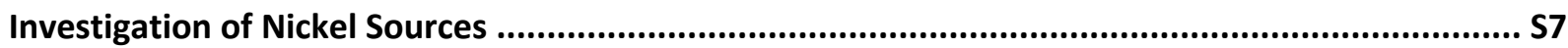

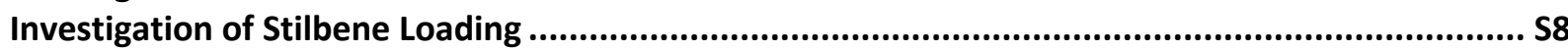

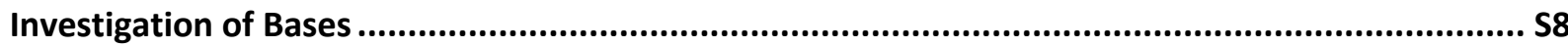

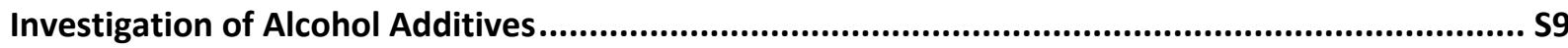

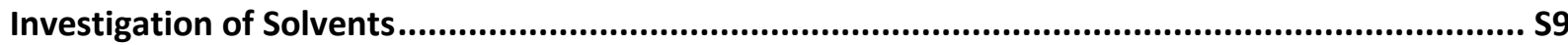

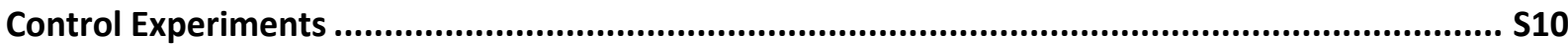

Investigation of Other Ligands Under Optimized Conditions .................................................. S10

Investigation of Equivalents of Ar-BPin...................................................................................... S11

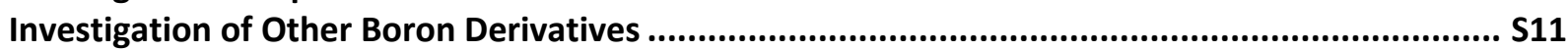

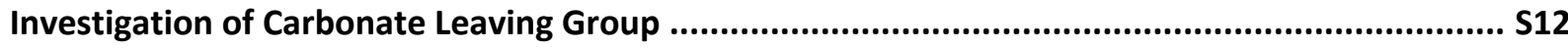

Representative Limitations in Substrate Scope ............................................... S13

Stereospecific Arylation of Tertiary Carboxylates........................................... S15

General Procedure A: Stereospecific Arylation of Tertiary Benzylic Pivalates ............................. S15

1 mmol Scale Cross-Coupling ...................................................................................................... S27

Preparation of Tertiary Benzylic Pivalates ....................................................... S37

General Procedure B: Preparation of Enantioenriched Tertiary Benzyl Pivalates .......................... S37

Preparation of Tertiary Benzylic Alcohols...................................................... S46

General Procedure C: Preparation of Enantioenriched Tertiary Benzyl Alcohols .......................... S47

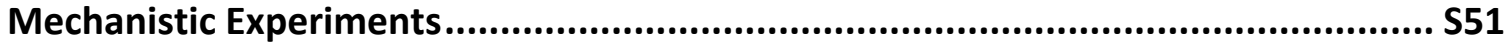

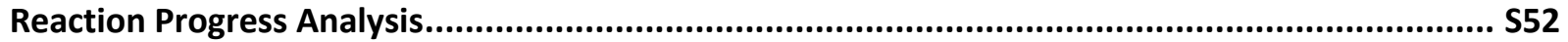

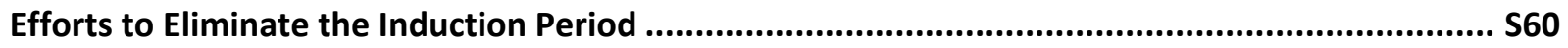

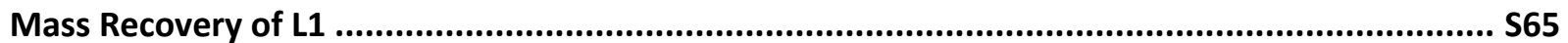

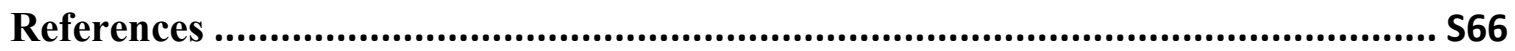

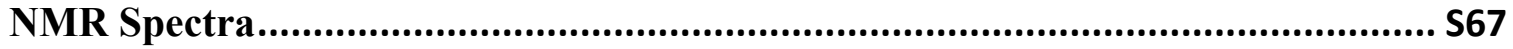

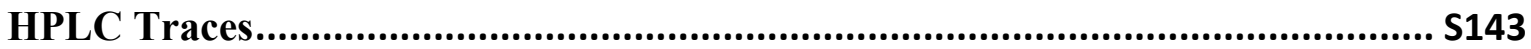




\section{General Information}

Reactions were performed in oven-dried vials with Teflon-lined caps or in oven-dried roundbottomed flasks unless otherwise noted. Flasks were fitted with rubber septa, and reactions were conducted under a positive pressure of $\mathrm{N}_{2}$. Stainless steel syringes were used to transfer air- and moisture-sensitive liquids. Flash chromatography was performed on silica gel 60 (40-63 $\mu \mathrm{m}$, or 5$20 \mu \mathrm{m} 60 \AA$ ) unless otherwise noted. Commercial reagents were purchased from Sigma Aldrich, Acros, Fisher, Strem, TCI, Combi Blocks, Alfa Aesar, or Cambridge Isotopes Laboratories and used as received with the following exceptions: cyclohexane (Sure Seal, under $\mathrm{N}_{2}$ ), sec-butyl alcohol (Sure Seal, under $\mathrm{N}_{2}$ ), lithium tert-butoxide, diethyl zinc, and titanium isopropoxide were purchased from vendors and immediately placed in a $\mathrm{N}_{2}$-atmosphere glovebox for storage. Walsh ligand was prepared according to reported literature procedure. ${ }^{1}$ Oven-dried potassium carbonate was added into $\mathrm{CDCl}_{3}$ to remove trace amount of acid. Proton nuclear magnetic resonance spectra $\left({ }^{1} \mathrm{H} \mathrm{NMR}\right)$, carbon nuclear magnetic resonance spectra $\left({ }^{13} \mathrm{C} \mathrm{NMR}\right)$, fluorine nuclear magnetic resonance spectra $\left({ }^{19} \mathrm{~F}\right.$ NMR $)$ and silicon nuclear magnetic resonance spectra $\left({ }^{29} \mathrm{Si} \mathrm{NMR}\right)$ were recorded on both $400 \mathrm{MHz}$ and $600 \mathrm{MHz}$ spectrometers. Chemical shifts for protons are reported in parts per million downfield from tetramethylsilane and are referenced to residual protium in the NMR solvent $\left(\mathrm{CHCl}_{3}=\delta 7.26\right)$. Chemical shifts for carbon are reported in parts per million downfield from tetramethylsilane and are referenced to the carbon resonances of the solvent $\left(\mathrm{CDCl}_{3}=\delta 77.2,\left(\mathrm{CD}_{3}\right)_{2} \mathrm{CO}=\delta 206.3,29.8\right)$. Chemical shifts for fluorine were externally referenced to $\mathrm{CFCl}_{3}$ in $\mathrm{CDCl}_{3}\left(\mathrm{CFCl}_{3}=\delta 0\right)$. Chemical shifts for silicon were externally referenced to tetramethylsilane in $\mathrm{CDCl}_{3}(\mathrm{TMS}=\delta 0$ ). Data are represented as follows: chemical shift, multiplicity ( $\mathrm{s}=$ singlet, $\mathrm{d}=$ doublet, $\mathrm{t}=$ triplet, $\mathrm{q}=$ quartet, $\mathrm{m}=$ multiplet), coupling constants in Hertz (Hz). The mass spectral data were obtained at the University of Delaware facilities. Optical rotations were measured using a $2.5 \mathrm{~mL}$ cell with a $0.1 \mathrm{dm}$ path length. Enantiomeric excess (ee) was determined using chiral HPLC analysis at the University of Delaware. Preparative chiral SFC was performed by Lotus Separations, Inc. 


\section{Optimization Studies}

\section{General Procedure for Optimization Studies}

In a $\mathrm{N}_{2}$-atmosphere glovebox, $\mathrm{Ni}$ source $(0.010 \mathrm{mmol}, 10 \mathrm{~mol} \%)$, ligand $(0.010 \mathrm{mmol}, 10 \mathrm{~mol}$ $\%)$, pivalate $(0.10 \mathrm{mmol}, 1.0$ equiv), boronate $(0.20 \mathrm{mmol}, 2.0$ equiv), $\mathrm{LiO} t-\mathrm{Bu}$ (w/o alcohol additive, $0.20 \mathrm{mmol}, 2.0$ equiv; $\mathrm{w} /$ alcohol additive, $0.30 \mathrm{mmol}, 3.0$ equiv) were weighed into a 1dram vial fitted with a magnetic stirbar, followed by alcohol additive (if used, $0.10 \mathrm{mmol}, 1.0$ equiv) and $\mathrm{CyH}(0.5 \mathrm{~mL}, 0.2 \mathrm{M})$. The vial was capped with a Teflon-lined cap and removed from the glovebox. The mixture was stirred at $80{ }^{\circ} \mathrm{C}$ for $24 \mathrm{~h}$. The reaction mixture was cooled to room temperature and diluted with $\mathrm{Et}_{2} \mathrm{O}(\sim 2 \mathrm{~mL})$. The mixture was stirred for ca. $10 \mathrm{~min}$, and then filtered through a small plug of silica gel, which was then rinsed with $\mathrm{Et}_{2} \mathrm{O}(\sim 10 \mathrm{~mL})$. The resulting solution was concentrated. Internal standard (1,3,5-trimethoxybenzene) was added, followed by $\mathrm{CDCl}_{3}$. The NMR yield of cross-coupled product was determined by ${ }^{1} \mathrm{H}$ NMR spectroscopic analysis via comparing with internal standard. 


\section{Investigation of Alkene Additives}<smiles>CCOC(C)(O[14CH3])c1ccccc1</smiles>

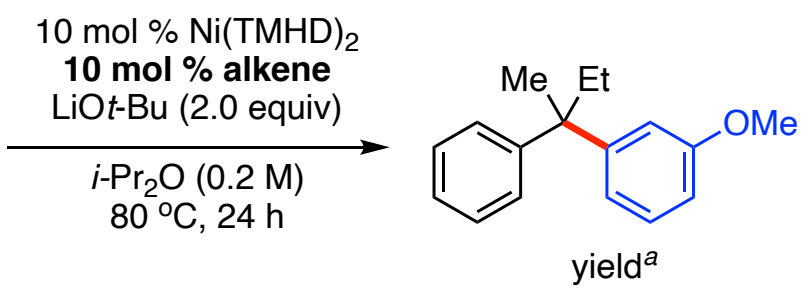

\section{A. Control}

no alkene

$47 \%$

\section{B. Stilbenes}<smiles>C(=C/c1ccccc1)\c1ccccc1</smiles><smiles></smiles><smiles>C(=C\c1ccccc1)\c1ccccc1</smiles><smiles>C(=C(c1ccccc1)c1ccccc1)c1ccccc1</smiles>

$41 \%$<smiles>O=C(c1ccccc1)C(c1ccccc1)c1ccccc1</smiles>

\section{Styrenes}

$\mathrm{F}_{3} \mathrm{C}$<smiles>C=Cc1cccc(F)c1</smiles>

$56 \%$<smiles>C=Cc1ccccn1</smiles>

$4 \%$<smiles>C=Cc1ccc(C(F)(F)F)cc1</smiles><smiles>C=Cc1ccc(F)cc1</smiles><smiles>C=Cc1ccc([N+](=O)[O-])cc1</smiles>

D. Fumarates<smiles>COC(=O)C=CC(C)=O</smiles><smiles>N#C/C=C/C#N</smiles><smiles>O=C1C=CC(=O)O1</smiles>

$14 \%$

$0 \%$<smiles>C=Cc1ccccc1</smiles><smiles>C=Cc1ccc(OC)cc1</smiles><smiles>CCc1ccccc1S(=O)(=O)O</smiles>

E. Dienes<smiles>C(/C=C/c1ccccc1)=C\c1ccccc1</smiles><smiles>CC(C)=CC=C(C)C</smiles><smiles>C=CC(=C)C</smiles>
$44 \%$ $55 \%$

$44 \%$

\section{F. Alkynes}

$\mathrm{Ph}=\mathrm{Ph}$

$33 \%$
$\mathrm{Ph}=\mathrm{Me}$

$43 \%$<smiles>O=C(/C=C/C(=O)N1[C@H]2c3ccccc3C[C@H]2CS1(=O)=O)N1C[C@@H]2Cc3ccccc3[C@H]21</smiles>

$16 \%$

Determined by ${ }^{1} \mathrm{H}$ NMR using 1,3,5-trimethoxybenzene as internal standard. 


\section{Investigation of Stilbenes in $i-\mathrm{Pr}_{2} \mathrm{O}$}
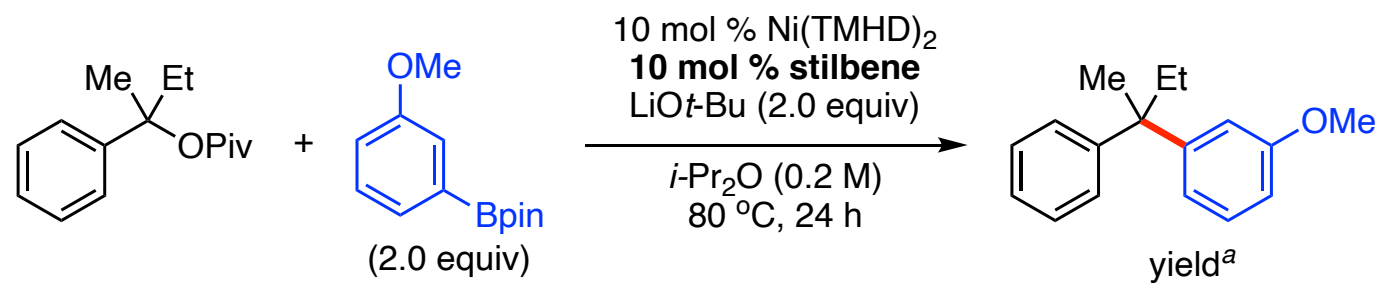<smiles>FC(F)(F)c1ccc(/C=C/c2ccc(C(F)(F)F)cc2)cc1</smiles><smiles>FC(F)(F)c1ccc(/C=C/c2ccccc2)cc1</smiles><smiles>C(=C/c1ccccc1)\c1ccccc1</smiles>

$51 \%$

$50 \%$

$81 \%$<smiles>COc1ccc(/C=C/c2ccc(/C=C/c3ccc(OC)cc3)cc2)cc1</smiles>

$72 \%$

$71 \%$

$86 \%$<smiles>COc1ccc(/C=C/c2ccc(C(F)(F)F)cc2)cc1</smiles>

$71 \%$<smiles>COc1ccccc1/C=C/c1ccccc1</smiles>

$80 \%$<smiles>C(=C/c1ccc2ccccc2c1)\c1ccc2ccccc2c1</smiles>

${ }^{a}$ Determined by ${ }^{1} \mathrm{H}$ NMR using 1,3,5-trimethoxybenzene as internal standard. 


\section{Investigation of Stilbenes in $\mathbf{C y H}$}

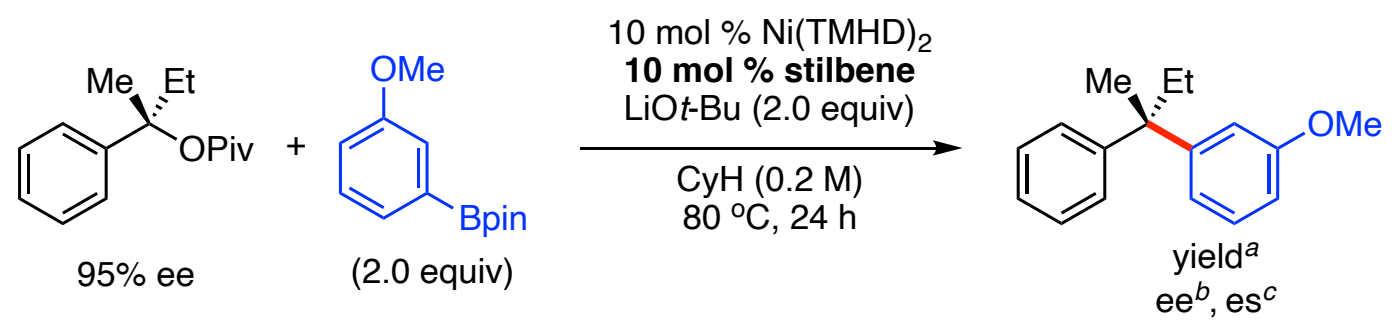<smiles>CCCOc1cc(/C=C/c2ccc(OCCC)c(/C=C/c3ccc(OC)cc3)c2)cc(OC(C)C)c1</smiles>

$57 \%$

$74 \%$ $86 \%$ ee, $91 \%$ es $88 \%$ ee, $93 \%$ es

$78 \%$

$85 \%$ ee, $89 \%$ es<smiles>COc1cc(/C=C/c2cc(OC)cc(OC)c2)cc(OC)c1</smiles>

$79 \%$

$88 \%$ ee, $93 \%$ es<smiles>COc1cccc(OC)c1/C=C/c1c(OC)cccc1OC</smiles>

$31 \%$ $84 \%$ ee, $88 \%$ es<smiles>Cc1cc(C)cc(/C=C/c2cc(C)cc(C)c2)c1</smiles>

$75 \%$ $86 \%$ ee, $91 \%$ es<smiles>Fc1cc(F)cc(/C=C/c2cc(F)cc(F)c2)c1</smiles><smiles>FC(F)(F)c1cc(/C=C/c2cc(C(F)(F)F)cc(C(F)(F)F)c2)cc(C(F)(F)F)c1</smiles>

${ }^{a}$ Determined by ${ }^{1} \mathrm{H}$ NMR using 1,3,5-trimethoxybenzene as internal standard. ${ }^{b}$ Determined by HPLC using a chiral stationary phase. ${ }^{c}$ es $=\left(\right.$ ee $\left._{\text {product }}\right) /\left(\mathrm{ee}_{\text {starting material }}\right)$. 


\section{Investigation of Nickel Sources}<smiles>CC[C@@](C)(O[N+])c1ccccc1</smiles>

$95 \%-96 \%$ ee<smiles>COc1cccc(Br)c1</smiles>

2.0 equiv
$10 \mathrm{~mol} \% \mathrm{Ni}$ source $10 \mathrm{~mol} \%$ stilbene-OMe

$\mathrm{LiOt}$-Bu (3.0 equiv) $s-\mathrm{BuOH}$ (1.0 equiv) $\mathrm{CyH}(0.2 \mathrm{M})$ $80^{\circ} \mathrm{C}, 24 \mathrm{~h}$

\begin{tabular}{ccccc}
\hline entry & $\mathrm{Ni} \mathrm{source}$ & ${\text { yield }(\%)^{a}}^{a}$ & ee $(\%)^{b}$ & es $(\%)^{c}$ \\
\hline 1 & $\mathrm{Ni}(\mathrm{COD})_{2}$ & 45 & 95 & 99 \\
2 & $\mathrm{NiCl}_{2} \cdot \mathrm{DME}$ & 73 & 96 & $>99$ \\
3 & $\mathrm{NiBr}_{2} \cdot \mathrm{DME}$ & 49 & 96 & $>99$ \\
4 & $\mathrm{Ni}(\mathrm{acac})_{2}$ & 66 & 96 & $>99$ \\
5 & $\mathrm{Ni}(\mathrm{TMHD})_{2}$ & 87 & 92 & 97 \\
6 & $\mathrm{Ni}(\mathrm{OTf})_{2}$ & 84 & 95 & 99 \\
7 & $\mathrm{Ni}(\mathrm{OAc})_{2} \cdot 4 \mathrm{H}_{2} \mathrm{O}$ & 88 & 96 & $>99$
\end{tabular}

${ }^{a}$ Determined by ${ }^{1} \mathrm{H}$ NMR using 1,3,5-trimethoxybenzene as internal standard. ${ }^{b}$ Determined by HPLC using a chiral stationary phase. ${ }^{c}$ es $=\left(\right.$ ee $\left._{\text {product }}\right) /\left(\right.$ ee $\left._{\text {starting material }}\right)$.
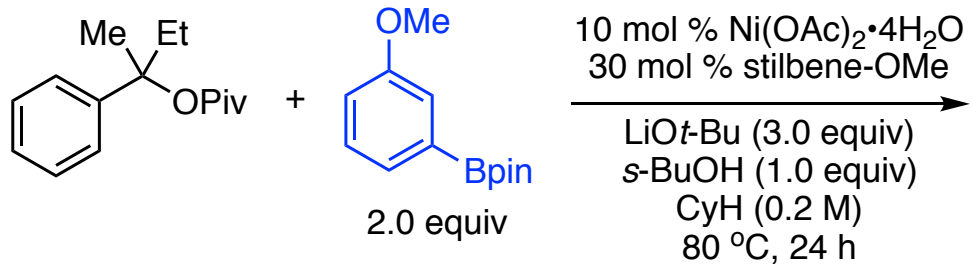<smiles>CCC(C)(c1ccccc1)c1cccc(OC)c1</smiles>
$80^{\circ} \mathrm{C}, 24 \mathrm{~h}$

\begin{tabular}{ccr}
\hline entry & deviation & yield $(\%)$ \\
\hline 1 & None & $>99$ \\
2 & $\mathrm{Ni}(\mathrm{COD})_{2}$ in lieu of $\mathrm{Ni}(\mathrm{OAc})_{2} \cdot 4 \mathrm{H}_{2} \mathrm{O}$ and stilbene-OMe & 18 \\
${ }^{a}$ Determined by ${ }^{1} \mathrm{H}$ NMR using & $1,3,5$-trimethoxybenzene as internal standard.
\end{tabular}


Investigation of Stilbene Loading
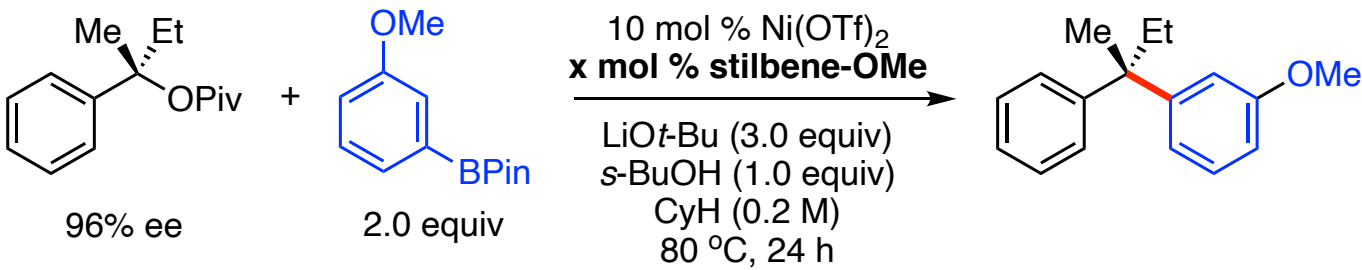

\begin{tabular}{ccccc}
\hline entry & mol \% stilbene-OMe & yield $(\%)^{a}$ & ee $(\%)^{b}$ & ${\text { es }(\%)^{c}}^{c}$ \\
\hline 1 & 0 & 16 & 90 & 94 \\
3 & 5 & 78 & 95 & 99 \\
4 & 10 & 84 & 95 & 99 \\
5 & 20 & 94 & 95 & 99 \\
6 & 30 & 90 & 96 & $>99$ \\
7 & 50 & 91 & 96 & $>99$ \\
$8^{d}$ & 100 & 93 & 96 & $>99$ \\
\hline
\end{tabular}

${ }^{a}$ Determined by ${ }^{1} \mathrm{H}$ NMR using 1,3,5-trimethoxybenzene as internal standard. ${ }^{b}$ Determined by HPLC using a chiral stationary phase. ${ }^{c}$ es $=($ ee product $) /\left(e_{\text {starting material }}\right) .{ }^{d} \mathrm{Ni}(\mathrm{OAc})_{2} \cdot 4 \mathrm{H}_{2} \mathrm{O}$ was used.

\section{Investigation of Bases}<smiles>CC[C@](C)(O[Na])c1ccccc1</smiles>

$96 \%$ ee<smiles>COc1cccc(Br)c1</smiles>

2.0 equiv
$10 \mathrm{~mol} \% \mathrm{Ni}(\mathrm{TMHD})_{2}$ $10 \mathrm{~mol} \%$ stilbene-OMe

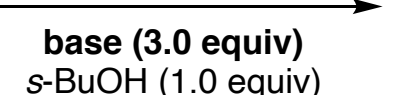

$\mathrm{CyH}(0.2 \mathrm{M})$ $80^{\circ} \mathrm{C}, 24 \mathrm{~h}$<smiles>CC[C@@](C)(c1ccccc1)c1cccc(OC)c1</smiles>

\begin{tabular}{ccccc}
\hline entry & base & ${\text { yield }(\%)^{a}}^{a}$ & ee $(\%)^{b}$ & ${\text { es }(\%)^{c}}^{b}$ \\
\hline 1 & $\mathrm{LiO} t-\mathrm{Bu}$ & 87 & 92 & 97 \\
2 & $\mathrm{NaO} t-\mathrm{Bu}$ & 7 & n.d. $^{d}$ & n.d. \\
3 & $\mathrm{KO} t$-Bu & 0 & - & - \\
4 & $\mathrm{LiOMe}$ & 0 & - & -
\end{tabular}

${ }^{a}$ Determined by ${ }^{1} \mathrm{H}$ NMR using 1,3,5-trimethoxybenzene as internal standard. ${ }^{b}$ Determined by HPLC using a chiral stationary phase. ${ }^{c} \mathrm{es}=\left(\mathrm{ee}_{\text {product }}\right) /\left(\mathrm{ee}_{\text {starting material }}\right) .{ }^{d}$ n.d. $=$ not determined. 
Investigation of Alcohol Additives

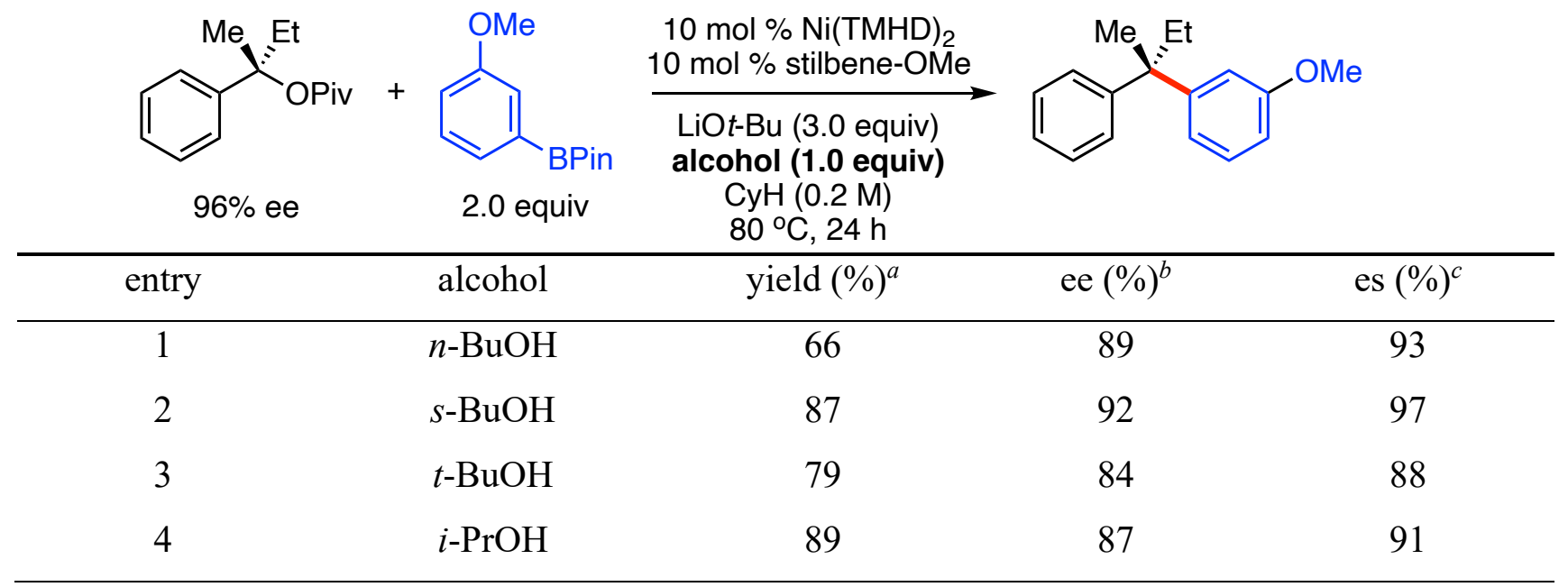

${ }^{a}$ Determined by ${ }^{1} \mathrm{H}$ NMR using 1,3,5-trimethoxybenzene as internal standard. ${ }^{b}$ Determined by HPLC using a chiral stationary phase. ${ }^{c}$ es $=\left(\right.$ ee $\left._{\text {product }}\right) /\left(\right.$ ee $\left._{\text {starting material }}\right)$.

\section{Investigation of Solvents}

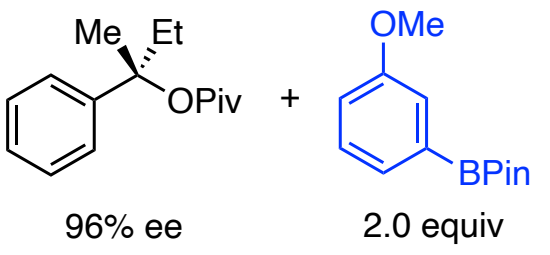

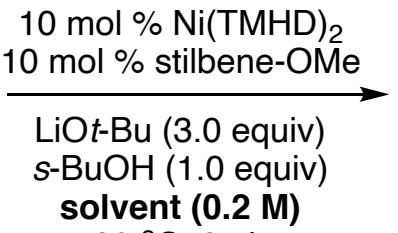
$80^{\circ} \mathrm{C}, 24 \mathrm{~h}$

\begin{tabular}{ccccc}
\hline entry & solvent & yield $(\%)^{a}$ & ee $(\%)^{b}$ & ${\text { es }(\%)^{c}}^{b}$ \\
\hline 1 & $2-\mathrm{Me}-\mathrm{THF}$ & 33 & 95 & 99 \\
2 & $i$-Pr $2 \mathrm{O}$ & 71 & 90 & 94 \\
3 & hexanes & 63 & 90 & 94 \\
4 & $\mathrm{CyH}$ & 87 & 92 & 97 \\
5 & $\mathrm{MeCN}$ & 0 & n.d. $^{d}$ & n.d. \\
\hline
\end{tabular}

${ }^{a}$ Determined by ${ }^{1} \mathrm{H}$ NMR using 1,3,5-trimethoxybenzene as internal standard. ${ }^{b}$ Determined by HPLC using a chiral stationary phase. ${ }^{c}$ es $=\left(e_{\text {product }}\right) /\left(\right.$ ee $\left._{\text {starting material }}\right) .{ }^{d}$ n.d. $=$ not determined. 


\section{Control Experiments}<smiles>CC[C@](C)(O[Na])c1ccccc1</smiles><smiles>COc1cccc(Br)c1</smiles>

2.0 equiv
$10 \mathrm{~mol} \% \mathrm{Ni}(\mathrm{OAc})_{2} \cdot 4 \mathrm{H}_{2} \mathrm{O}$

$30 \mathrm{~mol} \%$ stilbene-OMe

$\mathrm{LiOt}$-Bu (3.0 equiv)

$s-\mathrm{BuOH}$ (1.0 equiv)

$\mathrm{CyH}(0.2 \mathrm{M})$

$80^{\circ} \mathrm{C}, 24 \mathrm{~h}$

\begin{tabular}{ccccc}
\hline entry & deviation & yield $(\%)^{a}$ & ee $(\%)^{b}$ & es $(\%)^{c}$ \\
\hline 1 & without $\mathrm{Ni}(\mathrm{OAc})_{2} \cdot 4 \mathrm{H}_{2} \mathrm{O}$ & 0 & - & - \\
2 & without stilbene-OMe & 43 & 91 & 95 \\
3 & without $\mathrm{LiO} t-\mathrm{Bu}$ & 0 & - & - \\
4 & without $s$ - $\mathrm{BuOH}$ & 36 & 95 & 99 \\
\hline
\end{tabular}

${ }^{a}$ Determined by ${ }^{1} \mathrm{H}$ NMR using 1,3,5-trimethoxybenzene as internal standard. ${ }^{b}$ Determined by HPLC using a chiral stationary phase. ${ }^{c}$ es $=\left(e_{\text {product }}\right) /\left(e_{\text {starting material }}\right)$.

\section{Investigation of Other Ligands Under Optimized Conditions}

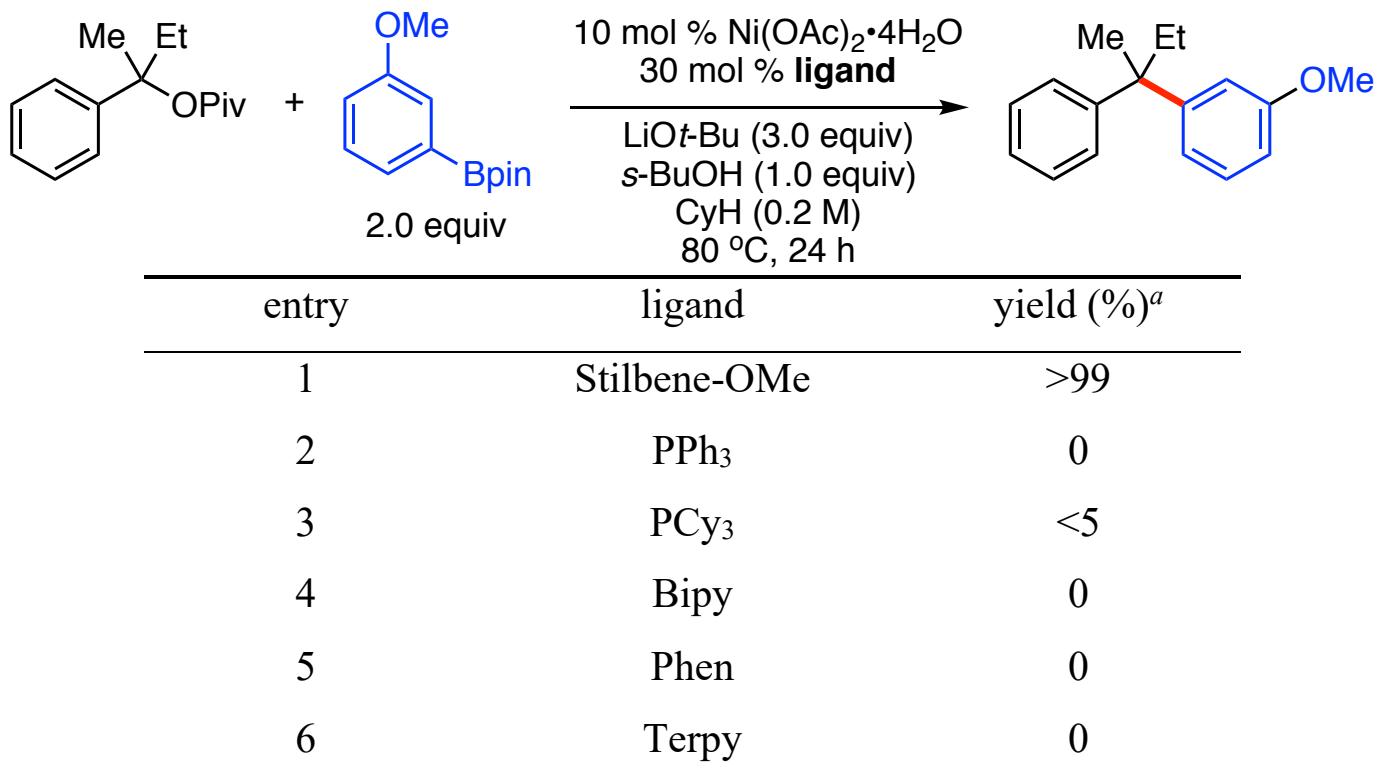

${ }^{a}$ Determined by ${ }^{1} \mathrm{H}$ NMR using 1,3,5-trimethoxybenzene as internal standard.

The use of $\mathrm{PCy}_{3}$ or CyJohnPhos results in the formation of alkene products from the pivalate, presumably via a $\beta$-hydride elimination pathway outcompeting the desired cross-coupling. These alkenes are not observed when stilbene-OMe is used. 


\section{Investigation of Equivalents of Ar-BPin}

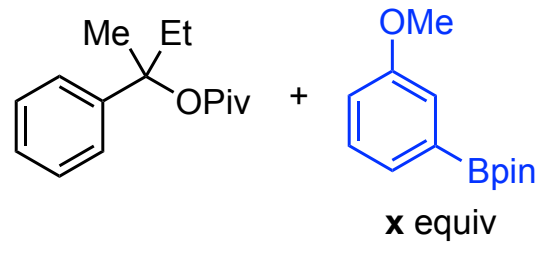

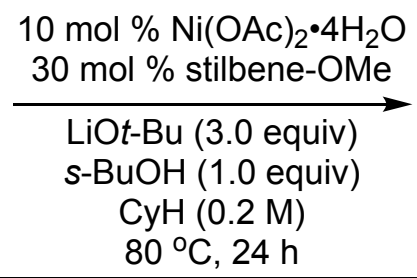

\begin{tabular}{ccc}
\hline entry & Equivalents of Ar-BPin & yield $(\%)^{a}$ \\
\hline 1 & 1.2 & 79 \\
2 & 1.5 & 83 \\
3 & 2.0 & $>99$
\end{tabular}

${ }^{a}$ Determined by ${ }^{1} \mathrm{H}$ NMR using 1,3,5-trimethoxybenzene as internal standard.

\begin{tabular}{|c|c|c|c|c|}
\hline entry & $\mathrm{Ar}-[\mathrm{B}]$ & yield $(\%)^{a}$ & ee $(\%)^{b}$ & $\operatorname{es}(\%)^{c}$ \\
\hline 1 & & 81 & 95 & 99 \\
\hline 2 & & 0 & - & - \\
\hline 3 & & 15 & - & - \\
\hline
\end{tabular}

\section{Investigation of Other Boron Derivatives}<smiles>CCOC(C)(O[Na])c1ccccc1</smiles>

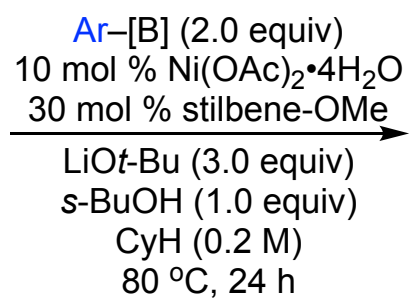
$80^{\circ} \mathrm{C}, 24 \mathrm{~h}$<smiles>CC[C@@](C)(c1ccccc1)c1cccc(OC)c1</smiles>

${ }^{a}$ Determined by ${ }^{1} \mathrm{H}$ NMR using 1,3,5-trimethoxybenzene as internal standard. ${ }^{b}$ Determined by HPLC using a chiral stationary phase. ${ }^{c}$ es $=\left(\right.$ ee $\left._{\text {product }}\right) /\left(e_{\text {starting material }}\right)$. 


\section{Investigation of Carbonate Leaving Group}

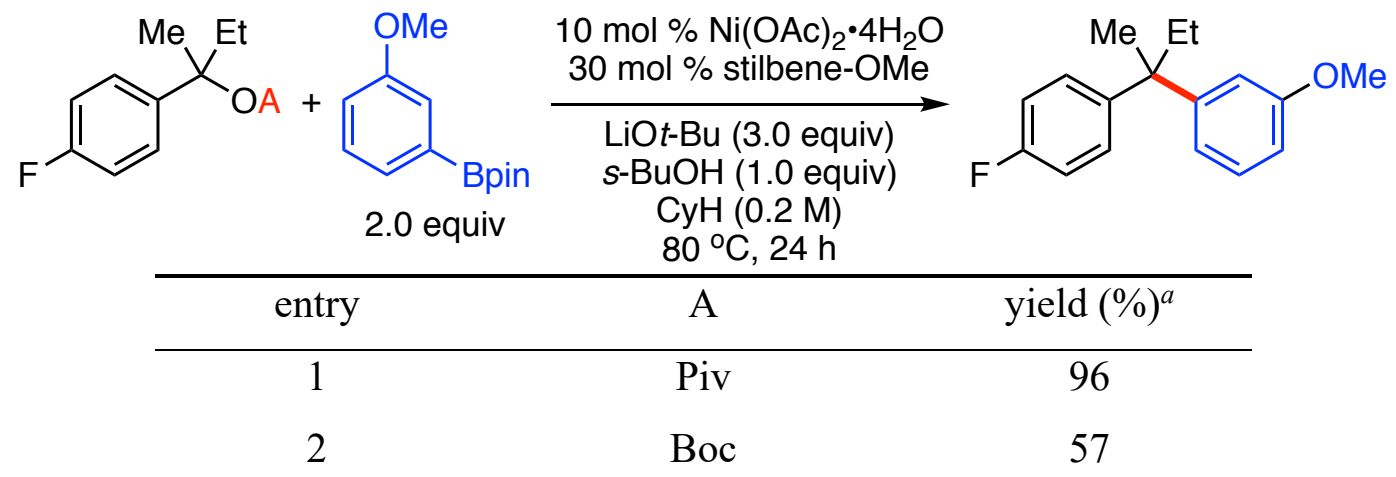

${ }^{a}$ Determined by ${ }^{1} \mathrm{H}$ NMR using 1,3,5-trimethoxybenzene as internal standard. 


\section{Representative Limitations in Substrate Scope}

Limitations in Pivalate Scope:

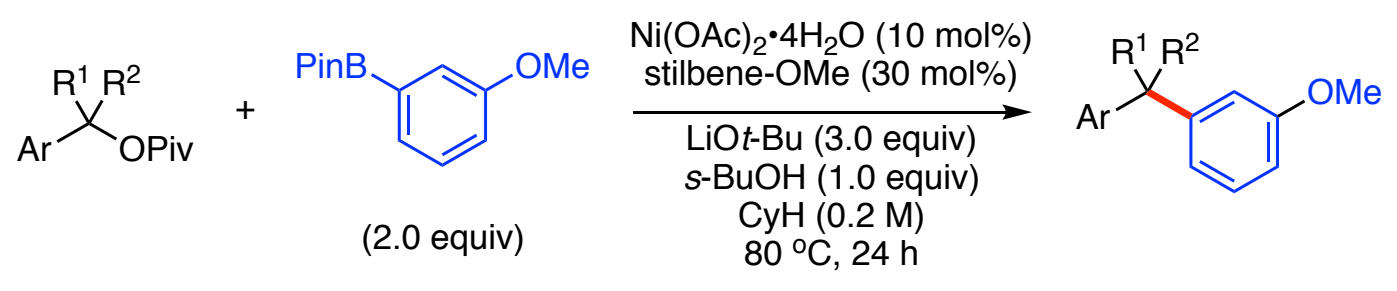<smiles>CC(F)(F)OC(C)(O[Te])c1ccccc1</smiles><smiles>CC(O[Na])c1ccccc1</smiles>

$$
\begin{array}{cc}
0 \% & <10 \% \\
\text { electron-poor substitution } & \text { secondary pivalate }
\end{array}
$$

Limitations in Boronate Scope:<smiles>CCC(C)(O[18F])c1ccccc1</smiles>

(2.0 equiv)

$$
\begin{gathered}
\begin{array}{c}
\mathrm{Ni}(\mathrm{OAc})_{2} \cdot 4 \mathrm{H}_{2} \mathrm{O}(10 \mathrm{~mol} \%) \\
\text { stilbene-OMe }(30 \mathrm{~mol} \%)
\end{array} \\
\stackrel{\mathrm{LiOt}-\mathrm{Bu}(3.0 \text { equiv) }}{\text { s-BuOH (1.0 equiv) }} \\
\mathrm{CyH}(0.2 \mathrm{M}) \\
80^{\circ} \mathrm{C}, 24 \mathrm{~h}
\end{gathered}
$$<smiles>CCC(C)(c1ccccc1)c1ccccc1</smiles><smiles>CC(=O)c1ccc(Br)cc1</smiles>

$0 \%$ NMR<smiles>COc1ccc(Br)cn1</smiles>

$32 \%$ NMR

hindrance

$\pi$ coordination

protic groups

heteroaryl

Conditions: pivalate $(0.10 \mathrm{mmol}), \mathrm{ArBpin}\left(2.0\right.$ equiv), $\mathrm{Ni}(\mathrm{OAc})_{2} \cdot 4 \mathrm{H}_{2} \mathrm{O}(10 \mathrm{~mol} \%)$, stilbeneOMe (30 mol \%), LiOt-Bu (3.0 equiv), s-BuOH (1.0 equiv), $\mathrm{CyH}(0.2 \mathrm{M}), 80^{\circ} \mathrm{C}, 24 \mathrm{~h}$. Yield was determined by ${ }^{1} \mathrm{H}$ NMR using 1,3,5-trimethoxybenzene as internal standard. 


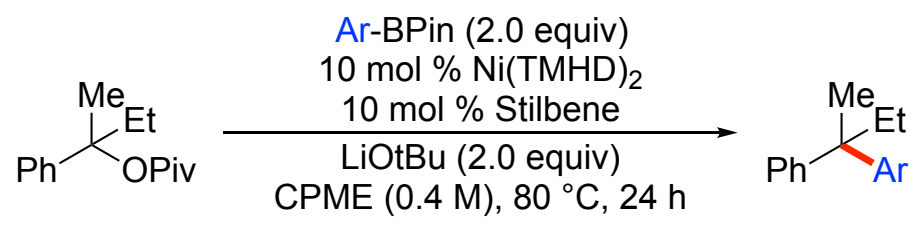

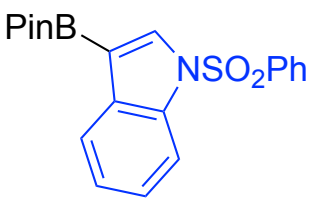

$0 \%(\mathrm{NMR})$

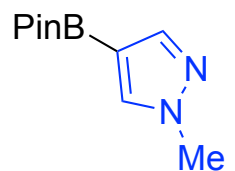

$0 \%(\mathrm{NMR})$

Conditions: pivalate $(0.10 \mathrm{mmol})$, ArBpin (2.0 equiv), Ni(TMHD) 2 (10 mol \%), stilbene (10 mol \%), $\mathrm{LiO} t$-Bu (2.0 equiv), $\mathrm{CPME}(0.4 \mathrm{M}), 80^{\circ} \mathrm{C}, 24 \mathrm{~h}$. Yield was determined by ${ }^{1} \mathrm{H}$ NMR using 1,3,5-trimethoxybenzene as internal standard.

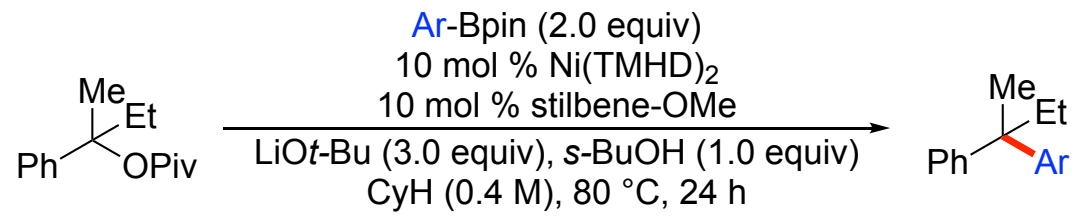

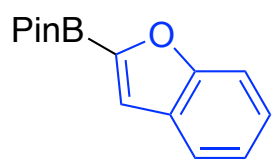

$0 \%(\mathrm{NMR})$

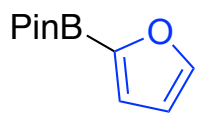

$0 \%(\mathrm{NMR})$

Conditions: pivalate $(0.10 \mathrm{mmol})$, ArBpin (2.0 equiv), Ni(TMHD) 2 (10 mol \%), stilbene-OMe (10 mol \%), $\mathrm{LiOt}$-Bu (3.0 equiv), $s-\mathrm{BuOH}$ (1.0 equiv), $\mathrm{CyH}(0.4 \mathrm{M}), 80{ }^{\circ} \mathrm{C}, 24 \mathrm{~h}$. Yield was determined by ${ }^{1} \mathrm{H}$ NMR using 1,3,5-trimethoxybenzene as internal standard. 


\section{Stereospecific Arylation of Tertiary Carboxylates}

\section{General Procedure A: Stereospecific Arylation of Tertiary Benzylic Pivalates}

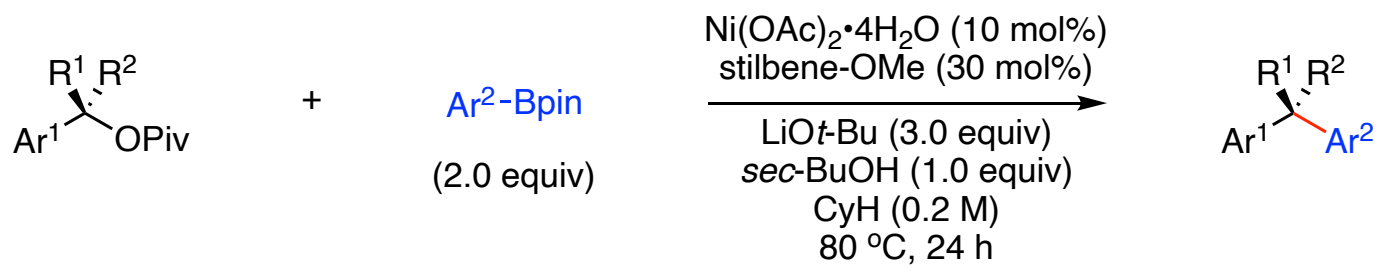

In a $\mathrm{N}_{2}$-atmosphere glovebox, $\mathrm{Ni}(\mathrm{OAc})_{2} \cdot 4 \mathrm{H}_{2} \mathrm{O}(10.0 \mathrm{mg}, 0.040 \mathrm{mmol}, 10 \mathrm{~mol} \%)$, stilbene-OMe (36.0 mg, $0.120 \mathrm{mmol}, 30 \mathrm{~mol} \%$ ), LiOt-Bu (96.1 mg, $1.20 \mathrm{mmol}, 3.0$ equiv), aryl boronate (0.80 mmol, 2.0 equiv) and tertiary benzylic pivalate $(0.40 \mathrm{mmol}, 1.0$ equiv) were weighed into a 1 dram vial fitted with a magnetic stirbar, followed by $s$-BuOH $(29.6 \mathrm{mg}, 37 \mu \mathrm{L}, 0.40 \mathrm{mmol}, 1.0$ equiv) and $\mathrm{CyH}(2.0 \mathrm{~mL}, 0.2 \mathrm{M})$. The vial was capped with a Teflon-lined cap and removed from the glovebox. The mixture was stirred at $80^{\circ} \mathrm{C}$ for $24 \mathrm{~h}$. The reaction mixture was cooled to room temperature and diluted with $\mathrm{Et}_{2} \mathrm{O}(\sim 2 \mathrm{~mL})$. The mixture was stirred for ca. $10 \mathrm{~min}$, and then filtered through a small plug of silica gel, which was then rinsed with $\mathrm{Et}_{2} \mathrm{O}(\sim 10 \mathrm{~mL})$. The resulting solution was concentrated. The cross-coupled product was then purified via silica gel chromatography. 


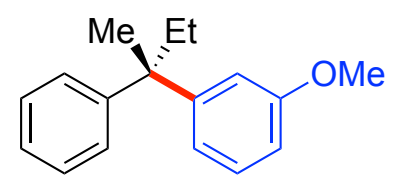

(S)-1-methoxy-3-(2-phenylbutan-2-yl)benzene (3). Prepared via General Procedure A using pivalate 1a $(96 \%$ ee). The crude mixture was purified by silica gel chromatography ( $5-40 \%$ $\mathrm{CH}_{2} \mathrm{Cl}_{2}$ /hexanes) to give 3 (run 1: $77.1 \mathrm{mg}, 80 \%$; run 2: $70.6 \mathrm{mg}, 73 \%$ ) as a colorless oil.

Enantiomeric Purity: 96\% ee (run 1: 96\% ee; run 2: 96\% ee) by chiral HPLC analysis (CHIRALPAK OD $-\mathrm{H}, 1.0 \mathrm{~mL} / \mathrm{min}, 1.0 \% i$-PrOH/hexanes, $\lambda=220 \mathrm{~nm}$ ); $t_{\mathrm{R}}$ (major) $=6.805 \mathrm{~min}$, $t_{\mathrm{R}}($ minor $)=8.733 \mathrm{~min}$.

${ }^{1} \mathbf{H}$ NMR (600 MHz, $\left.\mathbf{C D C l}_{3}\right) \delta 7.28-7.23(\mathrm{~m}, 2 \mathrm{H}), 7.22-7.14(\mathrm{~m}, 4 \mathrm{H}), 6.80-6.76(\mathrm{~m}, 2 \mathrm{H})$, $6.71(\mathrm{ddd}, J=8.1,2.5,1.0 \mathrm{~Hz}, 1 \mathrm{H}), 3.76(\mathrm{~s}, 3 \mathrm{H}), 2.13(\mathrm{q}, J=7.4 \mathrm{~Hz}, 2 \mathrm{H}), 1.60$ (s, 3H), 0.74 (t, $J$ $=7.4 \mathrm{~Hz}, 3 \mathrm{H})$.

${ }^{13}$ C NMR (151 MHz, $\left.\mathbf{C D C l}_{3}\right) \delta 159.4,151.6,149.6,128.9,128.0,127.5,125.7,120.4,114.3$, 110.2, 55.3, 46.7, 34.2, 27.0, 9.3.

HRMS (ESI+): $[\mathrm{M}+\mathrm{H}]^{+}$calculated for $\mathrm{C}_{17} \mathrm{H}_{21} \mathrm{O}: 241.1592$, found 241.1583 .

$[\alpha]_{\mathrm{D}}^{21.5}=-3.9^{\circ}\left(\mathrm{c} 1.45, \mathrm{CHCl}_{3}\right)$. lit. $(R)-\mathbf{3},[\boldsymbol{\alpha}]_{\mathbf{D}}^{21}=43^{\circ}\left(\mathrm{c} 0.1, \mathrm{CHCl}_{3}\right)$.

The spectral data matches that of the literature. ${ }^{2}$ 


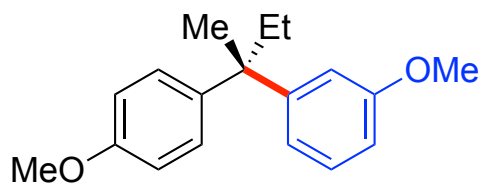

(S)-1-methoxy-3-(2-(4-methoxyphenyl)butan-2-yl)benzene (4). Prepared via General Procedure A using pivalate 1b $(92 \%$ ee). The crude mixture was purified by silica gel chromatography (5 - 40\% $\mathrm{CH}_{2} \mathrm{Cl}_{2} /$ hexanes) to give 4 (run 1: $93.7 \mathrm{mg}, 87 \%$; run 2: $92.4 \mathrm{mg}, 85 \%$ ) as a colorless oil.

Enantiomeric Purity: 90\% ee (run 1: $90 \%$ ee; run 2: 90\% ee) by chiral HPLC analysis (CHIRALPAK OD - H, $1.0 \mathrm{~mL} / \mathrm{min}, 1.0 \% i$-PrOH/hexanes, $\lambda=220 \mathrm{~nm}$ ); $t_{\mathrm{R}}($ major $)=9.451 \mathrm{~min}$, $t_{\mathrm{R}}(\operatorname{minor})=12.573 \mathrm{~min}$.

${ }^{1} \mathbf{H}$ NMR (600 MHz, $\left.\mathbf{C D C l}_{3}\right) \delta 7.21-7.15(\mathrm{~m}, 1 \mathrm{H}), 7.14-7.10(\mathrm{~m}, 2 \mathrm{H}), 6.83-6.76(\mathrm{~m}, 4 \mathrm{H})$, $6.73-6.67$ (m, 1H), 3.79 (s, 3H), 3.76 (s, 3H), 2.11 (q, $J=7.4 \mathrm{~Hz}, 2 \mathrm{H}), 1.58$ (s, 3H), 0.74 (t, $J=$ $7.3 \mathrm{~Hz}, 3 \mathrm{H})$.

${ }^{13}$ C NMR (151 MHz, $\left.\mathbf{C D C l}_{3}\right) \delta 159.4,157.5,151.9,141.8,128.8,128.5,120.3,114.2,113.3$, $110.1,55.3,55.2,46.0,34.3,27.2,9.4$.

HRMS (ESI+): $[\mathrm{M}+\mathrm{H}]^{+}$calculated for $\mathrm{C}_{18} \mathrm{H}_{23} \mathrm{O}_{2}: 271.1698$, found 271.1687.

$[\alpha]_{\mathrm{D}}^{21.6}=-3.2^{\circ}\left(\mathrm{c} 1.17, \mathrm{CHCl}_{3}\right)$. 


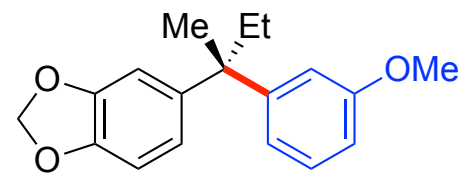

(R)-5-(2-(3-methoxyphenyl)butan-2-yl)benzo[d][1,3]dioxole (5). Prepared via General Procedure A using pivalate 1c $(92 \%$ ee). The crude mixture was purified by silica gel chromatography (5 - 40\% $\mathrm{CH}_{2} \mathrm{Cl}_{2} /$ hexanes) to give 5 (run 1: $104.3 \mathrm{mg}, 92 \%$; run 2: $107.7 \mathrm{mg}$, $95 \%)$ as a colorless oil.

Enantiomeric Purity: 92\% ee (run 1: 92\% ee; run 2: 93\% ee) by chiral HPLC analysis (CHIRALPAK OD $-\mathrm{H}, 1.0 \mathrm{~mL} / \mathrm{min}, 2.0 \% i$-PrOH/hexanes, $\lambda=220 \mathrm{~nm}$ ); $t_{\mathrm{R}}$ (major) $=7.014 \mathrm{~min}$, $t_{\mathrm{R}}($ minor $)=8.888 \mathrm{~min}$.

${ }^{1} \mathbf{H}$ NMR (600 MHz, $\left.\mathbf{C D C l}_{3}\right) \delta 7.18(\mathrm{t}, J=7.9 \mathrm{~Hz}, 1 \mathrm{H}), 6.80-6.75(\mathrm{~m}, 2 \mathrm{H}), 6.71$ (dtd, $J=5.8$, 2.9, $1.4 \mathrm{~Hz}, 3 \mathrm{H}), 6.64(\mathrm{~d}, J=1.6 \mathrm{~Hz}, 1 \mathrm{H}), 5.90$ (s, 2H), 3.77 (s, 3H), $2.13-2.01(\mathrm{~m}, 2 \mathrm{H}), 1.55$ (s, $3 \mathrm{H}), 0.74(\mathrm{t}, J=7.4 \mathrm{~Hz}, 3 \mathrm{H})$.

${ }^{13}$ C NMR (151 MHz, $\left.\mathbf{C D C l}_{3}\right) \delta 159.4,151.7,147.5,145.4,143.8,128.9,120.2,120.1,114.2$, 110.2, 108.6, 107.5, 100.9, 55.3, 46.5, 34.3, 27.2, 9.3.

HRMS (ESI+): $[\mathrm{M}+\mathrm{H}]^{+}$calculated for $\mathrm{C}_{18} \mathrm{H}_{21} \mathrm{O}_{3}: 285.1491$, found 285.1480 .

$[\alpha]_{\mathrm{D}}^{21.8}=+0.8^{\circ}\left(\mathrm{c} 1.24, \mathrm{CHCl}_{3}\right)$. 


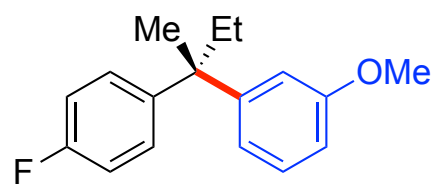

(S)-1-(2-(4-fluorophenyl)butan-2-yl)-3-methoxybenzene (6). Prepared via General Procedure A using pivalate 1d ( $91 \%$ ee). The crude mixture was purified by silica gel chromatography ( $2-20 \%$ $\mathrm{CH}_{2} \mathrm{Cl}_{2}$ /hexanes) to give 6 (run 1: $78.4 \mathrm{mg}, 76 \%$; run 2: $81.6 \mathrm{mg}, 79 \%$ ) as a colorless oil.

Enantiomeric Purity: 91\% ee (run 1: 91\% ee; run 2: 91\% ee) by chiral HPLC analysis (CHIRALPAK OD $-\mathrm{H}, 1.0 \mathrm{~mL} / \mathrm{min}, 1.0 \% i-\mathrm{PrOH} /$ hexanes, $\lambda=280 \mathrm{~nm}$ ); $t_{\mathrm{R}}($ major $)=6.246 \mathrm{~min}$, $t_{\mathrm{R}}(\operatorname{minor})=8.716 \mathrm{~min}$.

${ }^{1} \mathbf{H}$ NMR (600 MHz, CDCl $) \delta 7.19(\mathrm{t}, J=7.9 \mathrm{~Hz}, 1 \mathrm{H}), 7.17-7.12(\mathrm{~m}, 2 \mathrm{H}), 6.97-6.91(\mathrm{~m}, 2 \mathrm{H})$, $6.78-6.70(\mathrm{~m}, 3 \mathrm{H}), 3.76(\mathrm{~s}, 3 \mathrm{H}), 2.15-2.05(\mathrm{~m}, 2 \mathrm{H}), 1.58(\mathrm{~s}, 3 \mathrm{H}), 0.73(\mathrm{t}, J=7.4 \mathrm{~Hz}, 3 \mathrm{H})$.

${ }^{13}$ C NMR (151 MHz, $\left.\mathbf{C D C l}_{3}\right) \delta 161.0\left(\mathrm{~d}, J_{\mathrm{C}-\mathrm{F}}=244.1 \mathrm{~Hz}\right), 159.4,151.4,145.4\left(\mathrm{~d}, J_{\mathrm{C}-\mathrm{F}}=3.2 \mathrm{~Hz}\right)$, $129.01,128.97\left(\mathrm{~d}, J_{\mathrm{C}-\mathrm{F}}=2.3 \mathrm{~Hz}\right), 120.2,114.7\left(\mathrm{~d}, J_{\mathrm{C}-\mathrm{F}}=20.9 \mathrm{~Hz}\right), 114.3,110.3,55.3,46.3,34.3$, 27.2, 9.3.

${ }^{19}$ F NMR (565 MHz, $\left.\mathrm{CDCl}_{3}\right) \delta-118.2$.

HRMS (ESI+): $[\mathrm{M}+\mathrm{H}]^{+}$calculated for $\mathrm{C}_{17} \mathrm{H}_{20} \mathrm{FO}: 259.1498$, found 259.1489 .

$[\alpha]_{\mathbf{D}}^{21.8}=-2.4^{\circ}\left(\mathrm{c} 1.50, \mathrm{CHCl}_{3}\right)$. 


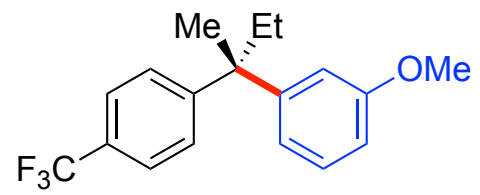

(S)-1-methoxy-3-(2-(4-(trifluoromethyl)phenyl)butan-2-yl)benzene (7). Prepared via General Procedure A using pivalate 1e $(88 \%$ ee). The crude mixture was purified by silica gel chromatography (2-20\% $\mathrm{CH}_{2} \mathrm{Cl}_{2} /$ hexanes) to give 7 (run 1: $84.8 \mathrm{mg}, 69 \%$; run 2: $74.9 \mathrm{mg}, 61 \%$ ) as a colorless oil.

Enantiomeric Purity: 88\% ee (run 1: $88 \%$ ee; run 2: $88 \%$ ee) by chiral HPLC analysis (CHIRALPAK OD - H, $1.0 \mathrm{~mL} / \mathrm{min}, 1.0 \% i$-PrOH/hexanes, $\lambda=210 \mathrm{~nm}$ ); $t_{\mathrm{R}}$ (major) $=6.891 \mathrm{~min}$, $t_{\mathrm{R}}($ minor $)=11.642 \mathrm{~min}$.

${ }^{1}$ H NMR (600 MHz, $\left.\mathbf{C D C l}_{3}\right) \delta 7.51(\mathrm{~d}, J=8.2 \mathrm{~Hz}, 2 \mathrm{H}), 7.31(\mathrm{~d}, J=8.2 \mathrm{~Hz}, 2 \mathrm{H}), 7.23-7.17(\mathrm{~m}$, $1 \mathrm{H}), 6.80-6.71(\mathrm{~m}, 3 \mathrm{H}), 3.77$ (s, 3H), 2.15 (q, $J=7.3 \mathrm{~Hz}, 2 \mathrm{H}), 1.61(\mathrm{~s}, 3 \mathrm{H}), 0.74$ (t, $J=7.3 \mathrm{~Hz}$, $3 \mathrm{H})$.

${ }^{13}$ C NMR (151 MHz, CDCl 3$) \delta 159.5,153.9,150.5,129.1,128.0$ (q, $\left.J_{\mathrm{C}-\mathrm{F}}=32.1 \mathrm{~Hz}\right), 127.8,125.0$ $\left(\mathrm{q}, J_{\mathrm{C}-\mathrm{F}}=3.7 \mathrm{~Hz}\right), 124.5\left(\mathrm{q}, J_{\mathrm{C}-\mathrm{F}}=271.7 \mathrm{~Hz}\right), 120.2,114.4,110.4,55.3,46.9,34.1,26.9,9.2$. ${ }^{19}$ F NMR (565 MHz, $\left.\mathrm{CDCl}_{3}\right) \delta-62.3$.

HRMS (ESI+): $[\mathrm{M}+\mathrm{H}]^{+}$calculated for $\mathrm{C}_{18} \mathrm{H}_{20} \mathrm{~F}_{3} \mathrm{O}: 309.1466$, found 309.1455. $[\alpha]_{\mathrm{D}}^{21.9}=-2.2^{\circ}\left(\mathrm{c} 1.20, \mathrm{CHCl}_{3}\right)$. 


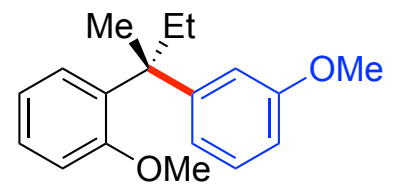

(R)-1-methoxy-2-(2-(3-methoxyphenyl)butan-2-yl)benzene (8). Prepared via General Procedure A using pivalate if $(90 \%$ ee). The crude mixture was purified by silica gel chromatography (5 - 40\% $\mathrm{CH}_{2} \mathrm{Cl}_{2} /$ hexanes) to give 8 (run 1: $74.2 \mathrm{mg}$, 69\%; run 2: $76.8 \mathrm{mg}, 71 \%$ ) as a colorless oil.

Enantiomeric Purity: 90\% ee (run 1: $90 \%$ ee; run 2: 90\% ee) by chiral HPLC analysis (CHIRALPAK OD - H, $1.0 \mathrm{~mL} / \mathrm{min}, 1.0 \% i$-PrOH/hexanes, $\lambda=220 \mathrm{~nm}$ ); $t_{\mathrm{R}}($ major $)=5.182 \mathrm{~min}$, $t_{\mathrm{R}}(\operatorname{minor})=7.005 \mathrm{~min}$.

${ }^{1} \mathrm{H}$ NMR (600 MHz, CDCl $) \delta 7.40(\mathrm{dd}, J=7.7,1.7 \mathrm{~Hz}, 1 \mathrm{H}), 7.22(\mathrm{ddd}, J=8.0,7.4,1.7 \mathrm{~Hz}, 1 \mathrm{H})$, $7.11(\mathrm{t}, J=7.9 \mathrm{~Hz}, 1 \mathrm{H}), 6.97$ (td, $J=7.5,1.3 \mathrm{~Hz}, 1 \mathrm{H}), 6.79$ (dd, $J=8.1,1.2 \mathrm{~Hz}, 1 \mathrm{H}), 6.75-6.68$ (m, 2H), 6.65 (ddd, $J=8.1,2.5,0.9 \mathrm{~Hz}, 1 \mathrm{H}), 3.75$ (s, 3H), 3.33 (s, 3H), 2.42 (dq, $J=12.8,7.4 \mathrm{~Hz}$, $1 \mathrm{H}), 2.00(\mathrm{dq}, J=12.9,7.3 \mathrm{~Hz}, 1 \mathrm{H}), 1.58(\mathrm{~s}, 3 \mathrm{H}), 0.67(\mathrm{t}, J=7.4 \mathrm{~Hz}, 3 \mathrm{H})$.

${ }^{13}$ C NMR (151 MHz, $\left.\mathbf{C D C l}_{3}\right) \delta 159.2,158.3,153.2,137.3,128.3,127.9,127.6,120.4,119.1$, $113.1,112.9,109.5,55.5,55.3,45.7,32.1,26.5,9.4$.

HRMS (ESI+): $[\mathrm{M}+\mathrm{H}]^{+}$calculated for $\mathrm{C}_{18} \mathrm{H}_{23} \mathrm{O}_{2}: 271.1698$, found 271.1686.

$[\alpha]_{\mathrm{D}}^{21.9}=+10.8^{\circ}\left(\mathrm{c} 1.53, \mathrm{CHCl}_{3}\right)$. 


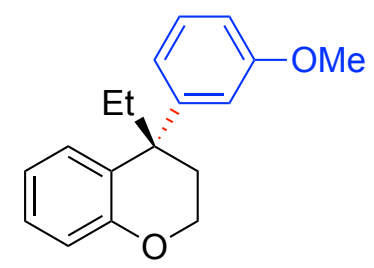

(S)-4-ethyl-4-(3-methoxyphenyl)chromane (9). Prepared via General Procedure A using pivalate 1g $(>99 \%$ ee). The crude mixture was purified by silica gel chromatography ( $5-40 \%$ $\mathrm{CH}_{2} \mathrm{Cl}_{2}$ /hexanes) to give 9 (run 1: $79.0 \mathrm{mg}, 74 \%$; run 2: $80.7 \mathrm{mg}, 75 \%$ ) as a colorless oil.

Enantiomeric Purity: 98\% ee (run 1: 98\% ee; run 2: 98\% ee) by chiral HPLC analysis (CHIRALPAK OD $-\mathrm{H}, 1.0 \mathrm{~mL} / \mathrm{min}, 1.0 \% i-\mathrm{PrOH} /$ hexanes, $\lambda=220 \mathrm{~nm}$ ); $t_{\mathrm{R}}$ (major) $=9.229 \mathrm{~min}$, $t_{\mathrm{R}}(\operatorname{minor})=11.005 \mathrm{~min}$.

${ }^{1} \mathbf{H}$ NMR (600 MHz, $\left.\mathbf{C D C l}_{3}\right) \delta 7.19(\mathrm{t}, J=8.0 \mathrm{~Hz}, 1 \mathrm{H}), 7.17-7.13(\mathrm{~m}, 1 \mathrm{H}), 7.10(\mathrm{dd}, J=7.8,1.6$ $\mathrm{Hz}, 1 \mathrm{H}), 6.93-6.88(\mathrm{~m}, 1 \mathrm{H}), 6.86(\mathrm{dd}, J=8.2,1.3 \mathrm{~Hz}, 1 \mathrm{H}), 6.75-6.71(\mathrm{~m}, 2 \mathrm{H}), 6.70(\mathrm{t}, J=2.1$ $\mathrm{Hz}, 1 \mathrm{H}), 4.15$ (dt, $J=10.9,3.8 \mathrm{~Hz}, 1 \mathrm{H}), 3.86$ (td, $J=11.2,2.2 \mathrm{~Hz}, 1 \mathrm{H}), 3.74$ (s, 3H), 2.36 (ddd, $J$ $=13.9,11.5,3.8 \mathrm{~Hz}, 1 \mathrm{H}), 2.25-2.11(\mathrm{~m}, 2 \mathrm{H}), 2.01(\mathrm{ddd}, J=13.9,3.9,2.2 \mathrm{~Hz}, 1 \mathrm{H}), 0.84(\mathrm{t}, J=$ $7.3 \mathrm{~Hz}, 3 \mathrm{H})$.

${ }^{13}$ C NMR (151 MHz, CDCl $) \delta 159.5,155.7,151.8,129.6,129.1,127.8,126.0,120.23,120.20$, $117.3,114.6,110.6,62.9,55.3,43.8,34.2,33.2,9.5$.

HRMS (ESI+): $[\mathrm{M}+\mathrm{H}]^{+}$calculated for $\mathrm{C}_{18} \mathrm{H}_{21} \mathrm{O}_{2}: 269.1542$, found 269.1531 . $[\alpha]_{\mathrm{D}}^{21.9}=-25.2^{\circ}\left(\mathrm{c} 1.36, \mathrm{CHCl}_{3}\right)$. 


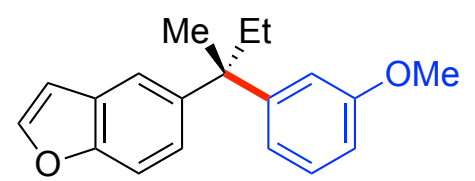

(S)-5-(2-(3-methoxyphenyl)butan-2-yl)benzofuran (10). Prepared via General Procedure A using pivalate $\mathbf{1 h}(95 \%$ ee). The crude mixture was purified by silica gel chromatography $(2-20 \%$ $\mathrm{CH}_{2} \mathrm{Cl}_{2} /$ hexanes) to give 10 (run 1: $101.0 \mathrm{mg}, 90 \%$; run 2: $100.0 \mathrm{mg}, 89 \%$ ) as a colorless oil.

Enantiomeric Purity: 95\% ee (run 1: 95\% ee; run 2: 95\% ee) by chiral HPLC analysis (CHIRALPAK OD - H, $1.0 \mathrm{~mL} / \mathrm{min}, 1.0 \% i$-PrOH/hexanes, $\lambda=220 \mathrm{~nm}$ ); $t_{\mathrm{R}}($ major $)=7.791 \mathrm{~min}$, $t_{\mathrm{R}}(\operatorname{minor})=11.278 \mathrm{~min}$.

${ }^{1} \mathbf{H}$ NMR (600 MHz, CDCl $) \delta 7.58(\mathrm{~d}, J=2.2 \mathrm{~Hz}, 1 \mathrm{H}), 7.49(\mathrm{~d}, J=1.9 \mathrm{~Hz}, 1 \mathrm{H}), 7.36(\mathrm{dt}, J=8.7$, $0.8 \mathrm{~Hz}, 1 \mathrm{H}), 7.18$ (t, $J=8.2 \mathrm{~Hz}, 1 \mathrm{H}), 7.08$ (dd, $J=8.7,2.0 \mathrm{~Hz}, 1 \mathrm{H}), 6.80$ (dtd, $J=4.9,2.2,1.3 \mathrm{~Hz}$, 2H), 6.72 (dtd, $J=4.6,2.5,1.0 \mathrm{~Hz}, 2 \mathrm{H}), 3.75$ (s, 3H), 2.19 (q, $J=7.3 \mathrm{~Hz}, 2 \mathrm{H}), 1.65$ (s, 3H), 0.75 (t, $J=7.3 \mathrm{~Hz}, 3 \mathrm{H})$.

${ }^{13}$ C NMR (151 MHz, $\left.\mathbf{C D C l}_{3}\right) \delta 159.4,153.3,152.1,145.1,144.3,128.9,127.0,124.7,120.3$, $119.5,114.3,110.8,110.1,106.9,55.3,46.7,34.5,27.5,9.4$.

HRMS (ESI+): $[\mathrm{M}+\mathrm{H}]^{+}$calculated for $\mathrm{C}_{19} \mathrm{H}_{21} \mathrm{O}_{2}: 281.1542$, found 281.1536 .

$[\boldsymbol{\alpha}]_{\mathrm{D}}^{21.9}=+1.4^{\circ}\left(\mathrm{c} 1.11, \mathrm{CHCl}_{3}\right)$. 


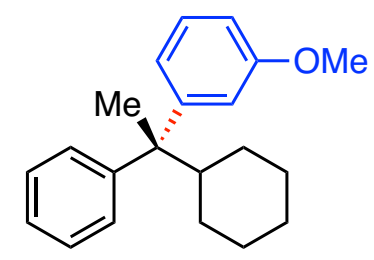

(R)-1-(1-cyclohexyl-1-phenylethyl)-3-methoxybenzene (11). Prepared via General Procedure A using pivalate $1 \mathbf{i}(95 \%$ ee). The crude mixture was purified by silica gel chromatography $(2-20 \%$ $\mathrm{CH}_{2} \mathrm{Cl}_{2} /$ hexanes) to give $\mathbf{1 1}$ (63.8 $\mathrm{mg}, 54 \%$ ) as a colorless oil.

Enantiomeric Purity: 95\% ee by chiral HPLC analysis (CHIRALPAK OD - H, $1.0 \mathrm{~mL} / \mathrm{min}, 1.0 \%$ $i$-PrOH/hexanes, $\lambda=220 \mathrm{~nm}$ ); $t_{\mathrm{R}}($ major $)=5.554 \mathrm{~min}, t_{\mathrm{R}}($ minor $)=4.974 \mathrm{~min}$.

${ }^{1} \mathbf{H}$ NMR (600 MHz, CDCl$) \delta 7.24(\mathrm{~d}, J=4.2 \mathrm{~Hz}, 4 \mathrm{H}), 7.19-7.11(\mathrm{~m}, 2 \mathrm{H}), 6.85-6.79(\mathrm{~m}, 2 \mathrm{H})$, 6.69 (dd, $J=8.2,2.4 \mathrm{~Hz}, 1 \mathrm{H}), 3.76(\mathrm{~s}, 3 \mathrm{H}), 2.22(\mathrm{tt}, J=11.6,2.5 \mathrm{~Hz}, 1 \mathrm{H}), 1.76-1.70(\mathrm{~m}, 2 \mathrm{H})$, $1.69-1.58(\mathrm{~m}, 6 \mathrm{H}), 1.30$ (qt, $J=12.4,3.0 \mathrm{~Hz}, 2 \mathrm{H}), 1.09$ (qt, $J=13.0,3.9 \mathrm{~Hz}, 1 \mathrm{H}), 0.98-0.87$ (m, 2H).

${ }^{13}$ C NMR (151 MHz, $\left.\mathbf{C D C l}_{3}\right) \delta 159.3,150.6,148.5,128.7,127.87,127.86,125.5,120.7,114.8$, $109.8,55.2,49.6,45.7,29.03,29.01,27.42,27.40,27.0,23.2$.

HRMS (ESI+): $[\mathrm{M}+\mathrm{H}]^{+}$calculated for $\mathrm{C}_{21} \mathrm{H}_{27} \mathrm{O}: 295.2062$, found 295.2051 .

$[\alpha]_{\mathrm{D}}^{21.5}=+1.5^{\circ}\left(\mathrm{c} 0.62, \mathrm{CHCl}_{3}\right)$. 


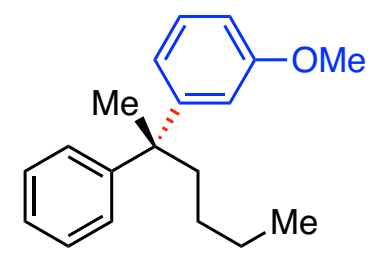

(R)-1-methoxy-3-(2-phenylhexan-2-yl)benzene (12). Prepared via General Procedure A using pivalate $1 \mathbf{j}$ ( $96 \%$ ee). The crude mixture was purified by silica gel chromatography $(2-20 \%$ $\mathrm{CH}_{2} \mathrm{Cl}_{2} /$ hexanes) to give $\mathbf{1 2}$ (96.5 mg, $90 \%$ ) as a colorless oil.

Enantiomeric Purity: $96 \%$ ee by chiral HPLC analysis (CHIRALPAK OJ - H, $1.0 \mathrm{~mL} / \mathrm{min}, 3.0 \%$ $i$-PrOH/hexanes, $\lambda=210 \mathrm{~nm}) ; t_{\mathrm{R}}($ major $)=10.059 \mathrm{~min}, t_{\mathrm{R}}(\operatorname{minor})=7.352 \mathrm{~min}$.

${ }^{1} \mathbf{H}$ NMR (600 MHz, $\left.\mathbf{C D C l}_{3}\right) \delta 7.28-7.23(\mathrm{~m}, 2 \mathrm{H}), 7.22-7.13(\mathrm{~m}, 4 \mathrm{H}), 6.79-6.76(\mathrm{~m}, 2 \mathrm{H})$, 6.71 (ddd, $J=8.2,2.4,1.1 \mathrm{~Hz}, 1 \mathrm{H}), 3.76$ (s, 3H), $2.14-2.00$ (m, 2H), $1.62(\mathrm{~s}, 3 \mathrm{H}), 1.29$ (dt, $J=$ 14.6, $7.3 \mathrm{~Hz}, 2 \mathrm{H}), 1.11-1.03(\mathrm{~m}, 2 \mathrm{H}), 0.85(\mathrm{t}, J=7.3 \mathrm{~Hz}, 3 \mathrm{H})$.

${ }^{13}$ C NMR (151 MHz, $\left.\mathbf{C D C l}_{3}\right) \delta 159.4,151.9,149.9,128.9,128.0,127.4,125.7,120.3,114.2$, $110.1,55.3,46.4,41.7,27.7,27.1,23.6,14.2$.

HRMS (ESI+): $[\mathrm{M}+\mathrm{H}]^{+}$calculated for $\mathrm{C}_{19} \mathrm{H}_{25} \mathrm{O}: 269.1905$, found 269.1895 .

$[\boldsymbol{\alpha}]_{\mathbf{D}}^{21.8}=+2.8^{\circ}\left(\mathrm{c} 1.24, \mathrm{CHCl}_{3}\right)$. 


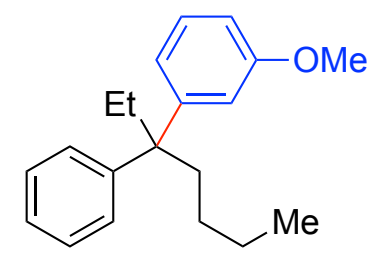

1-methoxy-3-(3-phenylheptan-3-yl)benzene (13). Prepared via General Procedure A using pivalate 1k. The crude mixture was purified by silica gel chromatography $(2-20 \%$ $\mathrm{CH}_{2} \mathrm{Cl}_{2} /$ hexanes) to give $\mathbf{1 3}$ (76.6 $\mathrm{mg}, 68 \%$ ) as a colorless oil.

${ }^{1} \mathbf{H}$ NMR (600 MHz, $\left.\mathbf{C D C l}_{3}\right) \delta 7.26-7.23(\mathrm{~m}, 2 \mathrm{H}), 7.20-7.12(\mathrm{~m}, 4 \mathrm{H}), 6.79-6.73(\mathrm{~m}, 2 \mathrm{H})$, $6.72-6.68(\mathrm{~m}, 1 \mathrm{H}), 3.76(\mathrm{~s}, 3 \mathrm{H}), 2.12(\mathrm{q}, J=7.3 \mathrm{~Hz}, 2 \mathrm{H}), 2.08-1.99(\mathrm{~m}, 2 \mathrm{H}), 1.32-1.21(\mathrm{~m}$, 2H), $0.99-0.90$ (m, 2H), 0.83 (t, $J=7.4 \mathrm{~Hz}, 3 \mathrm{H}), 0.64$ (t, $J=7.3 \mathrm{~Hz}, 3 \mathrm{H})$.

${ }^{13} \mathbf{C}$ NMR (151 MHz, $\left.\mathbf{C D C l}_{3}\right) \delta$ 159.2, 151.0, 148.9, 128.6, 128.1, 127.8, 125.5, 121.0, 114.9, $109.9,55.2,49.7,36.7,30.0,26.2,23.5,14.2,8.6$.

HRMS (ESI+): $[\mathrm{M}+\mathrm{H}]^{+}$calculated for $\mathrm{C}_{20} \mathrm{H}_{27} \mathrm{O}: 283.2062$, found 283.2049 . 


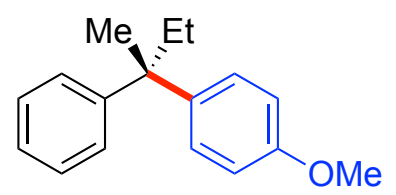

(S)-1-methoxy-4-(2-phenylbutan-2-yl)benzene (14). Prepared via General Procedure A using pivalate 1a $(96 \%$ ee). The crude mixture was purified by silica gel chromatography ( $5-40 \%$ $\mathrm{CH}_{2} \mathrm{Cl}_{2}$ /hexanes) to give $\mathbf{1 4}$ (run 1: $72.9 \mathrm{mg}, 76 \%$; run 2: $73.1 \mathrm{mg}, 76 \%$ ) as a colorless oil.

Enantiomeric Purity: 96\% ee (run 1: 96\% ee; run 2: 96\% ee) by chiral HPLC analysis (CHIRALPAK OD $-\mathrm{H}, 1.0 \mathrm{~mL} / \mathrm{min}, 1.0 \% i-\mathrm{PrOH} / \mathrm{h}$ exanes, $\lambda=220 \mathrm{~nm}$ ); $t_{\mathrm{R}}($ major $)=12.419 \mathrm{~min}$, $t_{\mathrm{R}}(\operatorname{minor})=14.870 \mathrm{~min}$.

${ }^{1} \mathbf{H}$ NMR (600 MHz, $\left.\mathbf{C D C l}_{3}\right) \delta 7.28-7.24(\mathrm{~m}, 2 \mathrm{H}), 7.22-7.18(\mathrm{~m}, 2 \mathrm{H}), 7.16$ (ddt, $J=8.5,6.8$, $1.3 \mathrm{~Hz}, 1 \mathrm{H}), 7.13-7.09(\mathrm{~m}, 2 \mathrm{H}), 6.83-6.79(\mathrm{~m}, 2 \mathrm{H}), 3.79(\mathrm{~s}, 3 \mathrm{H}), 2.15-2.09(\mathrm{~m}, 2 \mathrm{H}), 1.59(\mathrm{~s}$, $3 \mathrm{H}), 0.74(\mathrm{t}, J=7.4 \mathrm{~Hz}, 3 \mathrm{H})$.

${ }^{13}$ C NMR (151 MHz, $\left.\mathbf{C D C l}_{3}\right) \delta 157.5,150.1,142.0,128.6,128.0,127.5,125.6,113.3,55.3,46.0$, 34.3, 27.2, 9.3.

HRMS (LIFDI+): [M] $]^{+}$calculated for $\mathrm{C}_{17} \mathrm{H}_{20} \mathrm{O}: 240.1514$, found 240.1518 .

$[\alpha]_{\mathrm{D}}^{20.3}=-0.4^{\circ}\left(\mathrm{c} 1.36, \mathrm{CHCl}_{3}\right)$.

\section{1 mmol Scale Cross-Coupling}

Product 11 was also prepared on a 1-mmol scale. In a $\mathrm{N}_{2}$-atmosphere glovebox, $\mathrm{Ni}(\mathrm{OAc})_{2} \cdot 4 \mathrm{H}_{2} \mathrm{O}$ (24.9 mg, $0.10 \mathrm{mmol}, 10 \mathrm{~mol} \%$ ), stilbene-OMe (90.1 mg, $0.30 \mathrm{mmol}, 30 \mathrm{~mol} \%$ ), LiOt-Bu (240.2 $\mathrm{mg}, 3.00 \mathrm{mmol}, 3.0$ equiv), aryl boronate ( $468.2 \mathrm{mg}, 2.00 \mathrm{mmol}, 2.0$ equiv) and tertiary benzylic pivalate (234.3 mg, $1.00 \mathrm{mmol}, 1.0$ equiv, $96 \%$ ee) were weighed into a 2-dram vial fitted with a magnetic stirbar, followed by $s-\mathrm{BuOH}(74.1 \mathrm{mg}, 92 \mu \mathrm{L}, 1.00 \mathrm{mmol}, 1.0$ equiv) and $\mathrm{CyH}(5.0 \mathrm{~mL}$, $0.2 \mathrm{M}$ ). The vial was capped with a Teflon-lined cap and removed from the glovebox. The mixture was stirred at $80{ }^{\circ} \mathrm{C}$ for $24 \mathrm{~h}$. The reaction mixture was cooled to room temperature and diluted with $\mathrm{Et}_{2} \mathrm{O}(\sim 5 \mathrm{~mL})$. The mixture was stirred for ca. $10 \mathrm{~min}$, and then filtered through a small plug of silica gel, which was then rinsed with $\mathrm{Et}_{2} \mathrm{O}(\sim 30 \mathrm{~mL})$. The resulting solution was concentrated. The crude mixture was purified by silica gel chromatography (5-40\% $\mathrm{CH}_{2} \mathrm{Cl}_{2} /$ hexanes) to give 14 (185.7 $\mathrm{mg}, 77 \%, 96 \%$ ee) as a colorless oil. 


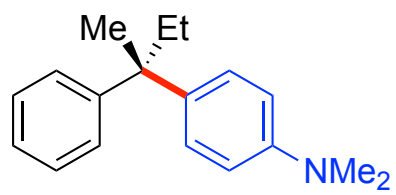

(S)-N,N-dimethyl-4-(2-phenylbutan-2-yl)aniline (15). Prepared via General Procedure A using pivalate 1a $(96 \%$ ee). The crude mixture was purified by silica gel chromatography (10 - 80\% $\mathrm{CH}_{2} \mathrm{Cl}_{2}$ /hexanes) to give 15 (run 1: $65.5 \mathrm{mg}, 65 \%$; run 2: $53.2 \mathrm{mg}, 52 \%$ ) as a colorless oil.

Enantiomeric Purity: 96\% ee (run 1: 96\% ee; run 2: 96\% ee) by chiral HPLC analysis (CHIRALPAK OD $-\mathrm{H}, 1.0 \mathrm{~mL} / \mathrm{min}, 1.0 \% i$-PrOH/hexanes, $\lambda=254 \mathrm{~nm}$ ); $t_{\mathrm{R}}($ major $)=5.840 \mathrm{~min}$, $t_{\mathrm{R}}($ minor $)=5.320 \mathrm{~min}$.

${ }^{1} \mathbf{H}$ NMR (600 MHz, $\left.\mathbf{C D C l}_{3}\right) \delta 7.28-7.19(\mathrm{~m}, 4 \mathrm{H}), 7.15$ (ddt, $\left.J=8.5,7.6,1.5 \mathrm{~Hz}, 1 \mathrm{H}\right), 7.09-$ $7.04(\mathrm{~m}, 2 \mathrm{H}), 6.69-6.64(\mathrm{~m}, 2 \mathrm{H}), 2.92(\mathrm{~s}, 6 \mathrm{H}), 2.11(\mathrm{q}, J=7.4 \mathrm{~Hz}, 2 \mathrm{H}), 1.58(\mathrm{~s}, 3 \mathrm{H}), 0.74(\mathrm{t}, J=$ $7.3 \mathrm{~Hz}, 3 \mathrm{H})$.

${ }^{13}$ C NMR (151 MHz, $\left.\mathbf{C D C l}_{3}\right) \delta 150.4,148.6,137.9,128.2,127.9,127.6,125.4,112.3,45.8,40.8$, 34.3, 27.2, 9.4.

HRMS (ESI+): $[\mathrm{M}+\mathrm{H}]^{+}$calculated for $\mathrm{C}_{18} \mathrm{H}_{24} \mathrm{~N}: 254.1909$, found 254.1899 .

$[\boldsymbol{\alpha}]_{\mathrm{D}}^{20.5}=+1.4^{\circ}\left(\mathrm{c} 1.22, \mathrm{CHCl}_{3}\right)$. 


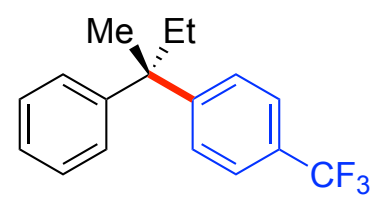

(S)-1-(2-phenylbutan-2-yl)-4-(trifluoromethyl)benzene (16). Prepared via General Procedure A using pivalate 1a ( $96 \%$ ee). The crude mixture was purified by silica gel chromatography ( $0-2 \%$ $\mathrm{CH}_{2} \mathrm{Cl}_{2}$ /hexanes) to give $\mathbf{1 6}$ (run 1: $76.9 \mathrm{mg}, 69 \%$; run 2: 75.8mg, 68\%) as a colorless oil.

Enantiomeric Purity: 95\% ee (run 1: 95\% ee; run 2: 95\% ee) by chiral HPLC analysis (CHIRALPAK OD $-\mathrm{H}, 1.0 \mathrm{~mL} / \mathrm{min}, 1.0 \% i$-PrOH/hexanes, $\lambda=220 \mathrm{~nm}$ ); $t_{\mathrm{R}}$ (major) $=5.422 \mathrm{~min}$, $t_{\mathrm{R}}(\operatorname{minor})=4.741 \mathrm{~min}$.

${ }^{1} \mathbf{H}$ NMR (600 MHz, $\left.\mathbf{C D C l}_{3}\right) \delta 7.52(\mathrm{~d}, J=8.4 \mathrm{~Hz}, 2 \mathrm{H}), 7.34-7.24(\mathrm{~m}, 4 \mathrm{H}), 7.22-7.16(\mathrm{~m}, 3 \mathrm{H})$, $2.23-2.12(\mathrm{~m}, 2 \mathrm{H}), 1.64(\mathrm{~s}, 3 \mathrm{H}), 0.75(\mathrm{t}, J=7.4 \mathrm{~Hz}, 3 \mathrm{H})$.

${ }^{13}$ C NMR (151 MHz, $\left.\mathbf{C D C l}_{3}\right) \delta 154.1,148.8,128.2,128.0\left(\mathrm{q}, J_{\mathrm{C}-\mathrm{F}}=32.2 \mathrm{~Hz}\right), 127.9,127.5,126.1$, $125.0\left(\mathrm{q}, J_{\mathrm{C}-\mathrm{F}}=3.8 \mathrm{~Hz}\right), 124.5\left(\mathrm{q}, J_{\mathrm{C}-\mathrm{F}}=271.6 \mathrm{~Hz}\right), 46.9,34.1,27.0,9.2$.

${ }^{19}$ F NMR (565 MHz, $\left.\mathrm{CDCl}_{3}\right) \delta-62.3$.

HRMS (LIFDI+): [M] $]^{+}$calculated for $\mathrm{C}_{17} \mathrm{H}_{17} \mathrm{~F}_{3}: 278.1282$, found 278.1279 .

$[\boldsymbol{\alpha}]_{\mathrm{D}}^{20.7}=-1.4^{\circ}\left(\mathrm{c} 1.29, \mathrm{CHCl}_{3}\right)$. 


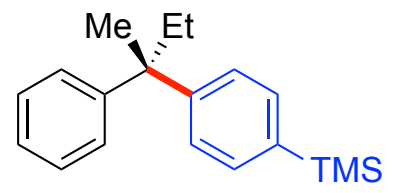

(S)-trimethyl(4-(2-phenylbutan-2-yl)phenyl)silane (17). Prepared via General Procedure A using pivalate 1a ( $96 \%$ ee). The crude mixture was purified by silica gel chromatography $(0-2 \%$ $\mathrm{CH}_{2} \mathrm{Cl}_{2}$ /hexanes) to give 17 (run 1: $93.3 \mathrm{mg}, 83 \%$; run 2: $93.7 \mathrm{mg}, 83 \%$ ) as a colorless oil.

Enantiomeric Purity: 96\% ee (run 1: 96\% ee; run 2: 96\% ee) by chiral HPLC analysis (CHIRALPAK OJ $-\mathrm{H}, 0.2 \mathrm{~mL} / \mathrm{min}, 3.0 \% i$-PrOH/hexanes, $\lambda=220 \mathrm{~nm}$ ); $t_{\mathrm{R}}($ major $)=21.191 \mathrm{~min}$, $t_{\mathrm{R}}(\operatorname{minor})=19.294 \mathrm{~min}$.

${ }^{1} \mathbf{H}$ NMR (600 MHz, $\left.\mathbf{C D C l}_{3}\right) \delta 7.45-7.37(\mathrm{~m}, 2 \mathrm{H}), 7.30-7.24(\mathrm{~m}, 2 \mathrm{H}), 7.23-7.14(\mathrm{~m}, 5 \mathrm{H})$, 2.15 (q, $J=7.4 \mathrm{~Hz}, 2 \mathrm{H}), 1.61$ (s, 3H), $0.74(\mathrm{t}, J=7.4 \mathrm{~Hz}, 3 \mathrm{H}), 0.24$ (s, 9H).

${ }^{13} \mathbf{C}$ NMR (151 MHz, $\left.\mathbf{C D C l}_{3}\right) \delta 150.5,149.5,137.1,133.1,128.0,127.7,126.9,125.7,46.6,34.2$, 27.0, 9.4,-0.9.

${ }^{29} \mathrm{Si}$ NMR (119 MHz, $\left.\mathrm{CDCl}_{3}\right) \delta-4.6$.

HRMS (LIFDI+): [M] ${ }^{+}$calculated for $\mathrm{C}_{19} \mathrm{H}_{26} \mathrm{Si}: 282.1804$, found 282.1816 .

$[\boldsymbol{\alpha}]_{\mathrm{D}}^{20.8}=+5.1^{\circ}\left(\mathrm{c} 1.25, \mathrm{CHCl}_{3}\right)$. 


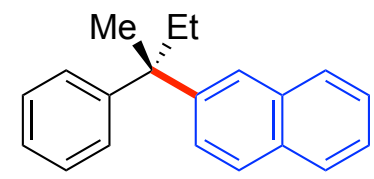

(S)-2-(2-phenylbutan-2-yl)naphthalene (18). Prepared via General Procedure A using pivalate 1a $(96 \%$ ee). The crude mixture was purified by silica gel chromatography ( $2-20 \%$ $\mathrm{CH}_{2} \mathrm{Cl}_{2}$ /hexanes) to give 18 (run 1: $80.3 \mathrm{mg}, 77 \%$; run 2: $87.4 \mathrm{mg}, 84 \%$ ) as a colorless oil.

Enantiomeric Purity: 96\% ee (run 1: 96\% ee; run 2: 96\% ee) by chiral HPLC analysis (CHIRALPAK OJ $-\mathrm{H}, 0.5 \mathrm{~mL} / \mathrm{min}, 10.0 \% i-\mathrm{PrOH} /$ hexanes, $\lambda=210 \mathrm{~nm}$ ); $t_{\mathrm{R}}$ (major) $=34.389 \mathrm{~min}$, $t_{\mathrm{R}}(\operatorname{minor})=40.750 \mathrm{~min}$.

${ }^{1} \mathbf{H}$ NMR (600 MHz, CDCl $) \delta 7.83(\mathrm{~d}, J=8.0 \mathrm{~Hz}, 1 \mathrm{H}), 7.80-7.76(\mathrm{~m}, 2 \mathrm{H}), 7.68(\mathrm{~d}, J=8.7 \mathrm{~Hz}$, 1H), $7.50-7.41(\mathrm{~m}, 2 \mathrm{H}), 7.29-7.21(\mathrm{~m}, 4 \mathrm{H}), 7.17(\mathrm{tt}, J=8.6,1.7 \mathrm{~Hz}, 2 \mathrm{H}), 2.26$ (qd, $J=7.4,4.0$ $\mathrm{Hz}, 2 \mathrm{H}), 1.71$ (s, 3H), 0.77 (t, $J=7.4 \mathrm{~Hz}, 3 \mathrm{H})$.

${ }^{13}$ C NMR (151 MHz, $\left.\mathbf{C D C l}_{3}\right) \delta 149.7,147.0,133.3,131.9,128.14,128.09,127.7,127.6,127.5$, $127.3,126.0,125.8,125.6,125.0,46.9,33.9,27.0,9.4$.

HRMS (LIFDI+): [M] calculated for $\mathrm{C}_{20} \mathrm{H}_{20}: 260.1565$, found 260.1577 .

$[\boldsymbol{\alpha}]_{\mathrm{D}}^{21.0}=-13.8^{\circ}\left(\mathrm{c} 1.19, \mathrm{CHCl}_{3}\right)$. lit. $(R)-\mathbf{1 7},[\boldsymbol{\alpha}]_{\mathrm{D}}^{24}=+13.3^{\circ}\left(\mathrm{c} 1.02, \mathrm{CHCl}_{3}\right)$.

The spectral data matches that of the literature. ${ }^{3}$ 


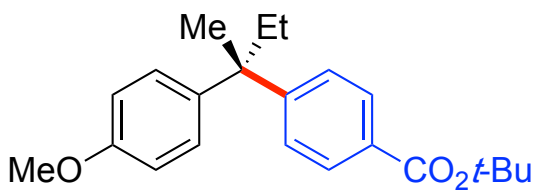

tert-butyl (R)-4-(2-(4-methoxyphenyl)butan-2-yl)benzoate (19). Prepared via General Procedure A using pivalate 1b $(92 \%$ ee). The crude mixture was purified by silica gel chromatography (10 - 100\% $\mathrm{CH}_{2} \mathrm{Cl}_{2} /$ hexanes) to give 19 (run 1: $118.4 \mathrm{mg}, 87 \%$; run 2: $117.0 \mathrm{mg}$, $86 \%)$ as a colorless oil.

Enantiomeric Purity: 90\% ee (run 1: $90 \%$ ee; run 2: 90\% ee) by chiral HPLC analysis (CHIRALPAK OD $-\mathrm{H}, 1.0 \mathrm{~mL} / \mathrm{min}, 1.0 \% i$-PrOH/hexanes, $\lambda=210 \mathrm{~nm}$ ); $t_{\mathrm{R}}$ (major) $=8.199 \mathrm{~min}$, $t_{\mathrm{R}}($ minor $)=7.261 \mathrm{~min}$.

${ }^{1} \mathbf{H}$ NMR (600 MHz, CDCl$) \delta 7.91-7.86(\mathrm{~m}, 2 \mathrm{H}), 7.26-7.23(\mathrm{~m}, 2 \mathrm{H}), 7.10-7.05(\mathrm{~m}, 2 \mathrm{H})$, $6.83-6.77(\mathrm{~m}, 2 \mathrm{H}), 3.78(\mathrm{~s}, 3 \mathrm{H}), 2.17-2.08(\mathrm{~m}, 2 \mathrm{H}), 1.59(\mathrm{~s}, 3 \mathrm{H}), 1.58(\mathrm{~s}, 9 \mathrm{H}), 0.73(\mathrm{t}, J=7.3$ $\mathrm{Hz}, 3 \mathrm{H})$.

${ }^{13}$ C NMR (151 MHz, CDCl $) \delta 166.0,157.7,155.0,141.3,129.5,129.2,128.5,127.4,113.5,80.8$, $55.3,46.3,34.2,28.4,27.1,9.3$.

HRMS (ESI+): $[\mathrm{M}+\mathrm{H}]^{+}$calculated for $\mathrm{C}_{22} \mathrm{H}_{29} \mathrm{O}_{3}: 341.2117$, found 341.2104 .

$[\alpha]_{\mathbf{D}}^{21.1}=-3.2^{\circ}\left(\mathrm{c} 1.09, \mathrm{CHCl}_{3}\right)$. 


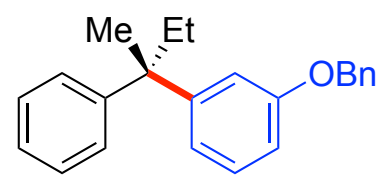

(S)-1-(benzyloxy)-3-(2-phenylbutan-2-yl)benzene (20). Prepared via General Procedure A using pivalate 1a $(96 \%$ ee). The crude mixture was purified by silica gel chromatography $(2-20 \%$ $\mathrm{CH}_{2} \mathrm{Cl}_{2} /$ hexanes) to give 20 (run 1: $96.3 \mathrm{mg}, 76 \%$; run 2: $88.0 \mathrm{mg}, 70 \%$ ) as a colorless oil.

Enantiomeric Purity: 96\% ee (run 1: $96 \%$ ee; run 2: 96\% ee) by chiral HPLC analysis (CHIRALPAK OD - H, $1.0 \mathrm{~mL} / \mathrm{min}, 1.0 \% i$-PrOH$/$ hexanes, $\lambda=210 \mathrm{~nm}$ ); $t_{\mathrm{R}}($ major $)=10.155 \mathrm{~min}$, $t_{\mathrm{R}}($ minor $)=12.142 \mathrm{~min}$.

${ }^{1}$ H NMR (600 MHz, CDCl $)$ ) 7.44 - 7.39 (m, 2H), 7.39 - 7.34 (m, 2H), $7.34-7.29$ (m, 1H), $7.29-7.23(\mathrm{~m}, 2 \mathrm{H}), 7.22-7.13(\mathrm{~m}, 4 \mathrm{H}), 6.86(\mathrm{t}, J=2.1 \mathrm{~Hz}, 1 \mathrm{H}), 6.79(\mathrm{dd}, J=8.0,2.7 \mathrm{~Hz}, 2 \mathrm{H})$, $5.00(\mathrm{~s}, 2 \mathrm{H}), 2.13$ (q, $J=7.3 \mathrm{~Hz}, 2 \mathrm{H}), 1.59$ (s, 3H), 0.73 (t, $J=7.3 \mathrm{~Hz}, 3 \mathrm{H})$.

${ }^{13}$ C NMR (151 MHz, CDCl $) \delta 158.7,151.6,149.6,137.3,128.9,128.7,128.0,127.8,127.5$, 125.7, 120.6, 115.2, 111.4, 70.2, 46.7, 34.2, 27.0, 9.3.

Coincident peaks were observed in the ${ }^{13} \mathrm{C}$ NMR spectrum when $\mathrm{CDCl}_{3}$ is used as solvent.

${ }^{13} \mathrm{C}$ NMR $\left(151 \mathrm{MHz},\left(\mathrm{CD}_{3}\right)_{2} \mathrm{CO}\right) \delta 159.6,152.3,150.4,138.5,129.7,129.2,128.7,128.6,128.5$, $128.1,126.4,120.9,115.7,112.4,70.4,47.2,34.6,27.1,9.5$.

HRMS (ESI+): [M+H] $]^{+}$calculated for $\mathrm{C}_{23} \mathrm{H}_{25} \mathrm{O}: 317.1905$, found 317.1894.

$[\alpha]_{\mathrm{D}}^{21.3}=-3.4^{\circ}\left(\mathrm{c} 1.44, \mathrm{CHCl}_{3}\right)$. 


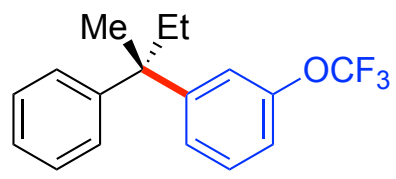

(S)-1-(2-phenylbutan-2-yl)-3-(trifluoromethoxy)benzene (21). Prepared via General Procedure A using pivalate 1a (96\% ee). The crude mixture was purified by silica gel chromatography ( $0-$ 2\% $\mathrm{CH}_{2} \mathrm{Cl}_{2} /$ hexanes) to give 21 (run 1: $81.4 \mathrm{mg}, 69 \%$; run 2: $89.7 \mathrm{mg}, 76 \%$ ) as a colorless oil.

Enantiomeric Purity: 95\% ee (run 1: 95\% ee; run 2: 95\% ee) by chiral HPLC analysis (CHIRALPAK OJ $-\mathrm{H}, 0.2 \mathrm{~mL} / \mathrm{min}, 3.0 \% i-\mathrm{PrOH} /$ hexanes, $\lambda=220 \mathrm{~nm}$ ); $t_{\mathrm{R}}($ major $)=22.651 \mathrm{~min}$, $t_{\mathrm{R}}($ minor $)=24.689 \mathrm{~min}$.

${ }^{1} \mathbf{H}$ NMR (600 MHz, $\left.\mathbf{C D C l}_{3}\right) \delta 7.30-7.23(\mathrm{~m}, 3 \mathrm{H}), 7.21-7.14(\mathrm{~m}, 3 \mathrm{H}), 7.11-7.00(\mathrm{~m}, 3 \mathrm{H})$, $2.19-2.07$ (m, 2H), 1.60 (s, 3H), $0.73(\mathrm{t}, J=7.4 \mathrm{~Hz}, 3 \mathrm{H})$.

${ }^{13}$ C NMR (151 MHz, CDCl $) \delta 152.5,149.3-149.2(\mathrm{~m}), 148.8,129.2,128.2,127.5,126.2,126.0$, $120.7\left(\mathrm{q}, J_{\mathrm{C}-\mathrm{F}}=256.6 \mathrm{~Hz}\right), 120.4,118.0,46.8,34.2,27.0,9.2$.

${ }^{19}$ F NMR (565 MHz, $\left.\mathrm{CDCl}_{3}\right) \delta-57.8$.

HRMS (LIFDI+): [M] $]^{+}$calculated for $\mathrm{C}_{17} \mathrm{H}_{17} \mathrm{~F}_{3} \mathrm{O}: 294.1231$, found 294.1245 .

$[\alpha]_{\mathrm{D}}^{21.4}=-0.2^{\circ}\left(\mathrm{c} 1.71, \mathrm{CHCl}_{3}\right)$. 


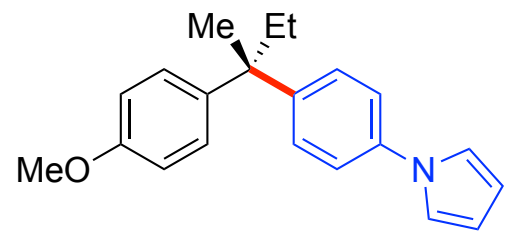

(R)-1-(4-(2-(4-methoxyphenyl)butan-2-yl)phenyl)-1H-pyrrole (22). Prepared via General Procedure A using pivalate 1b $(92 \%$ ee). The crude mixture was purified by silica gel chromatography (5 - 40\% $\mathrm{CH}_{2} \mathrm{Cl}_{2} /$ hexanes) to give 22 (run 1: $94.8 \mathrm{mg}, 78 \%$; run 2: $120.3 \mathrm{mg}$, $98 \%$ ) as a colorless oil.

Enantiomeric Purity: 92\% ee (run 1: $92 \%$ ee; run 2: 92\% ee) by chiral HPLC analysis (CHIRALPAK OJ $-\mathrm{H}, 1.0 \mathrm{~mL} / \mathrm{min}, 20.0 \% i-\mathrm{PrOH} / \mathrm{hexanes}, \lambda=254 \mathrm{~nm}$ ); $t_{\mathrm{R}}($ major) $=13.852 \mathrm{~min}$, $t_{\mathrm{R}}(\operatorname{minor})=12.029 \mathrm{~min}$.

${ }^{1} \mathbf{H}$ NMR (600 MHz, $\left.\mathbf{C D C l}_{3}\right) \delta 7.30-7.27(\mathrm{~m}, 2 \mathrm{H}), 7.27-7.22(\mathrm{~m}, 2 \mathrm{H}), 7.16-7.11(\mathrm{~m}, 2 \mathrm{H})$, $7.07(\mathrm{t}, J=2.2 \mathrm{~Hz}, 2 \mathrm{H}), 6.87-6.80(\mathrm{~m}, 2 \mathrm{H}), 6.33(\mathrm{t}, J=2.2 \mathrm{~Hz}, 2 \mathrm{H}), 3.80(\mathrm{~s}, 3 \mathrm{H}), 2.14$ (q, $J=7.3$ $\mathrm{Hz}, 2 \mathrm{H}), 1.61$ (s, 3H), 0.77 (t, $J=7.3 \mathrm{~Hz}, 3 \mathrm{H})$.

${ }^{13}$ C NMR (151 MHz, $\left.\mathbf{C D C l}_{3}\right) \delta 157.6,147.7,141.6,138.5,128.6,128.5,120.1,119.4,113.4$, 110.2, 55.3, 45.8, 34.4, 27.3, 9.4.

HRMS (ESI+): $[\mathrm{M}+\mathrm{H}]^{+}$calculated for $\mathrm{C}_{21} \mathrm{H}_{24} \mathrm{NO}: 306.1858$, found 306.1847 .

$[\boldsymbol{\alpha}]_{\mathrm{D}}^{21.5}=+0.7^{\circ}\left(\mathrm{c} 1.21, \mathrm{CHCl}_{3}\right)$. 


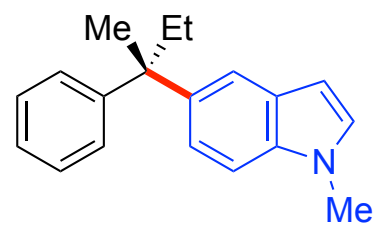

(S)-1-methyl-5-(2-phenylbutan-2-yl)-1H-indole (23). Prepared via General Procedure A using pivalate 1a $(96 \%$ ee). The crude mixture was purified by silica gel chromatography $(2-20 \%$ $\mathrm{CH}_{2} \mathrm{Cl}_{2}$ /hexanes) to give $\mathbf{2 3}$ (run 1: $78.4 \mathrm{mg}, 74 \%$; run 2: $76.2 \mathrm{mg}, 73 \%$ ) as a colorless oil.

Enantiomeric Purity: 96\% ee (run 1: 96\% ee; run 2: 96\% ee) by chiral HPLC analysis (CHIRALPAK OD $-\mathrm{H}, 1.0 \mathrm{~mL} / \mathrm{min}, 1.0 \% i-\mathrm{PrOH} / \mathrm{h}$ exanes, $\lambda=254 \mathrm{~nm}$ ); $t_{\mathrm{R}}$ (major) $=10.326 \mathrm{~min}$, $t_{\mathrm{R}}(\operatorname{minor})=9.052 \mathrm{~min}$.

${ }^{1} \mathbf{H}$ NMR (600 MHz, CDCl$) \delta 7.59(\mathrm{~d}, J=1.9 \mathrm{~Hz}, 1 \mathrm{H}), 7.29-7.25(\mathrm{~m}, 4 \mathrm{H}), 7.21(\mathrm{~d}, J=8.6 \mathrm{~Hz}$, 1H), $7.19-7.15(\mathrm{~m}, 1 \mathrm{H}), 7.04(\mathrm{~d}, J=3.1 \mathrm{~Hz}, 1 \mathrm{H}), 6.99$ (dd, $J=8.6,1.9 \mathrm{~Hz}, 1 \mathrm{H}), 6.47$ (dd, $J=$ $3.1,0.9 \mathrm{~Hz}, 1 \mathrm{H}), 3.78(\mathrm{~s}, 3 \mathrm{H}), 2.30-2.18(\mathrm{~m}, 2 \mathrm{H}), 1.69$ (s, 3H), $0.78(\mathrm{t}, J=7.3 \mathrm{~Hz}, 3 \mathrm{H})$.

${ }^{13}$ C NMR (151 MHz, $\left.\mathbf{C D C l}_{3}\right) \delta 151.0,140.7,135.1,128.8,128.1,127.9,127.7,125.4,122.6$, $119.0,108.8,101.2,46.6,34.6,32.9,27.6,9.5$.

HRMS (ESI+): $[\mathrm{M}+\mathrm{H}]^{+}$calculated for $\mathrm{C}_{19} \mathrm{H}_{22} \mathrm{~N}: 264.1752$, found 264.1743 .

$[\alpha]_{\mathrm{D}}^{21.5}=-5.7^{\circ}\left(\mathrm{c} 1.17, \mathrm{CHCl}_{3}\right)$. 


\section{Preparation of Tertiary Benzylic Pivalates}

Tertiary benzylic pivalates $\mathbf{1 a}, \mathbf{1 b}, \mathbf{1} \mathbf{c}$ and $\mathbf{1 g}$ were prepared as reported. ${ }^{4}$<smiles>CCOC(C)(CC)c1ccccc1</smiles>

$1 a$<smiles>CC[C@@](C)(O[Na])c1ccc(F)cc1</smiles>

1d<smiles>CC[C@]1(O)CCOc2ccccc21</smiles>

19<smiles>CCCC[C@](C)(O[Na])c1ccccc1</smiles>

1j<smiles>CC[C@@](C)(O[Na])c1ccc(OC)cc1</smiles>

$1 b$<smiles>CC[C@](C)(O[Na])c1ccc(C(F)(F)F)cc1</smiles>

$1 e$<smiles>CC[C@@](C)(O[Na])c1ccc2occc2c1</smiles>

$1 h$<smiles>CCCCC(O[Na])(O[Al])c1ccccc1</smiles>

$1 k$<smiles>CC[C@@](C)(O[Na])c1ccc2c(c1)OCO2</smiles>

$1 c$<smiles>CCO[C@@](C)(O[Na])c1ccccc1OC</smiles>

$1 f$<smiles>C[C@](O)(c1ccccc1)C1CCCCC1</smiles>

$1 \mathbf{i}$

\section{General Procedure B: Preparation of Enantioenriched Tertiary Benzyl Pivalates}

To an oven-dried, round-bottomed flask, KHMDS (1.0 equiv, 1.0 M in THF) was added dropwise to a solution of alcohol (1.0 equiv) and THF $([$ alcohol $]=0.5 \mathrm{M})$ under $\mathrm{N}_{2}$ at $0{ }^{\circ} \mathrm{C}$. The resulting solution was stirred for $30 \mathrm{~min}$ at $0{ }^{\circ} \mathrm{C}$. Then $\mathrm{PivCl}$ (1.0 equiv) was added dropwise. The solution was slowly warmed to r.t. and stirred for $4 \mathrm{~h}$. The mixture was then cooled by placing the flask in an ice-water bath. The reaction was quenched with saturated aqueous $\mathrm{NH}_{4} \mathrm{Cl}$, and the resulting mixture was extracted with $\mathrm{Et}_{2} \mathrm{O}$. The combined organic layers were washed with sat. $\mathrm{NaCl}$, dried $\left(\mathrm{Na}_{2} \mathrm{SO}_{4}\right)$, filtered, and concentrated. The residue was purified by silica gel chromatography (Et ${ }_{2} \mathrm{O} /$ hexanes). 


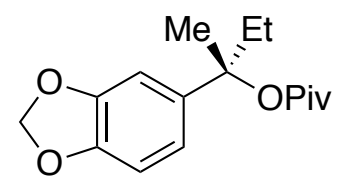

(S)-2-(benzo[d][1,3]dioxol-5-yl)butan-2-yl pivalate (1c). Prepared via General Procedure B. The crude mixture was purified by silica gel chromatography $\left(2-20 \% \mathrm{Et}_{2} \mathrm{O} /\right.$ hexanes) to give $1 \mathbf{c}$ ( 454.1 $\mathrm{mg}, 81 \%, 2.02 \mathrm{mmol}$ scale) as a colorless oil.

Enantiomeric Purity: 92\% ee by chiral HPLC analysis (CHIRALPAK OD - H, $1.0 \mathrm{~mL} / \mathrm{min}, 1.0 \%$ $i$-PrOH/hexanes, $\lambda=210 \mathrm{~nm}$ ); $t_{\mathrm{R}}$ (major) $=7.350 \mathrm{~min}, t_{\mathrm{R}}($ minor $)=9.705 \mathrm{~min}$.

${ }^{1}$ H NMR (600 MHz, CDCl $) \delta 6.81-6.79(\mathrm{~m}, 1 \mathrm{H}), 6.79-6.73(\mathrm{~m}, 2 \mathrm{H}), 5.94(\mathrm{~s}, 2 \mathrm{H}), 1.99$ (q, $J$ $=7.4 \mathrm{~Hz}, 2 \mathrm{H}), 1.75(\mathrm{~s}, 3 \mathrm{H}), 1.22(\mathrm{~s}, 9 \mathrm{H}), 0.80(\mathrm{t}, J=7.4 \mathrm{~Hz}, 3 \mathrm{H})$.

${ }^{13}$ C NMR (151 MHz, $\left.\mathbf{C D C l}_{3}\right) \delta 176.9,147.7,146.3,139.6,117.9,107.9,105.7,101.1,83.6,39.5$, $35.4,27.4,24.5,8.3$.

The spectral data matches that of the same compound prepared for our development of the related vinylation. ${ }^{4}$ 


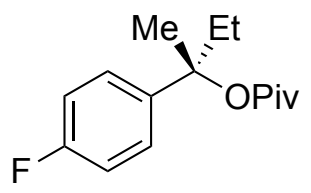

(S)-2-(4-fluorophenyl)butan-2-yl pivalate (1d). Prepared via General Procedure B. The crude mixture was purified by silica gel chromatography $\left(1-10 \% \mathrm{Et}_{2} \mathrm{O} /\right.$ hexanes $)$ to give $\mathbf{1 d}(510.8 \mathrm{mg}$, $93 \%, 2.18 \mathrm{mmol}$ scale) as a colorless oil.

Enantiomeric Purity: 92\% ee by chiral HPLC analysis (CHIRALPAK OJ - H, $1.0 \mathrm{~mL} / \mathrm{min}, 2.0 \%$ $i-\mathrm{PrOH} / \mathrm{hexanes}, \lambda=210 \mathrm{~nm})$; $t_{\mathrm{R}}($ major $)=5.395 \mathrm{~min}, t_{\mathrm{R}}($ minor $)=4.672 \mathrm{~min}$.

${ }^{1} \mathbf{H}$ NMR (600 MHz, CDCl $) \delta 8.30-7.24(\mathrm{~m}, 2 \mathrm{H}), 7.04-6.96(\mathrm{~m}, 2 \mathrm{H}), 2.09-1.96(\mathrm{~m}, 2 \mathrm{H})$, $1.78(\mathrm{~s}, 3 \mathrm{H}), 1.22(\mathrm{~s}, 9 \mathrm{H}), 0.80(\mathrm{t}, J=7.4 \mathrm{~Hz}, 3 \mathrm{H})$.

${ }^{13}$ C NMR (151 MHz, CDCl $) \delta 176.9,161.7\left(\mathrm{~d}, J_{\mathrm{C}-\mathrm{F}}=245.1 \mathrm{~Hz}\right), 141.1\left(\mathrm{~d}, J_{\mathrm{C}-\mathrm{F}}=3.4 \mathrm{~Hz}\right), 126.3$ $\left(\mathrm{d}, J_{\mathrm{C}-\mathrm{F}}=7.9 \mathrm{~Hz}\right), 115.0\left(\mathrm{~d}, J_{\mathrm{C}-\mathrm{F}}=21.2 \mathrm{~Hz}\right), 83.3,39.5,35.3,27.4,24.5,8.2$.

${ }^{19} \mathrm{~F}$ NMR (565 MHz, $\left.\mathrm{CDCl}_{3}\right) \delta-116.6$.

HRMS (EI+): $[\mathrm{M}]^{+}$calculated for $\mathrm{C}_{15} \mathrm{H}_{21} \mathrm{FO}_{2}: 252.1526$, found 252.1522 .

$[\alpha]_{\mathrm{D}}^{24.4}=-0.8^{\circ}\left(\mathrm{c} 1.15, \mathrm{CHCl}_{3}\right)$. 


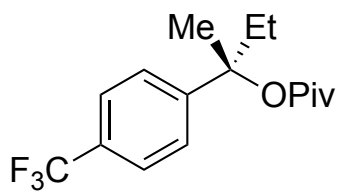

(S)-2-(4-(trifluoromethyl)phenyl)butan-2-yl pivalate (1e). Prepared via General Procedure B. The crude mixture was purified by silica gel chromatography $(2-20 \% \mathrm{DCM} /$ hexanes $)$ to give 1e (505.4 mg, 84\%, $2.0 \mathrm{mmol}$ scale) as a colorless oil.

Enantiomeric Purity: 88\% ee by chiral HPLC analysis (CHIRALPAK IA, $0.8 \mathrm{~mL} / \mathrm{min}, 100 \%$ hexanes, $\lambda=210 \mathrm{~nm}) ; t_{\mathrm{R}}($ major $)=5.634 \mathrm{~min}, t_{\mathrm{R}}($ minor $)=5.103 \mathrm{~min}$.

${ }^{1}$ H NMR (600 MHz, CDCl $) \delta 7.63-7.54(\mathrm{~m}, 2 \mathrm{H}), 7.43-7.36(\mathrm{~m}, 2 \mathrm{H}), 2.11-1.96(\mathrm{~m}, 2 \mathrm{H})$, $1.80(\mathrm{~s}, 3 \mathrm{H}), 1.24(\mathrm{~s}, 9 \mathrm{H}), 0.82(\mathrm{t}, J=7.4 \mathrm{~Hz}, 3 \mathrm{H})$.

${ }^{13}$ C NMR (151 MHz, CDCl $) \delta 176.9,149.5,129.1\left(\mathrm{q}, J_{\mathrm{C}-\mathrm{F}}=32.5 \mathrm{~Hz}\right), 125.3\left(\mathrm{q}, J_{\mathrm{C}-\mathrm{F}}=3.8 \mathrm{~Hz}\right)$, $125.0,124.3\left(\mathrm{q}, J_{\mathrm{C}-\mathrm{F}}=271.8 \mathrm{~Hz}\right), 83.1,39.5,35.3,27.3,24.4,8.1$.

${ }^{19}$ F NMR (565 MHz, CDCl 3 ) $\delta-62.5$.

HRMS (EI+): $[\mathrm{M}]^{+}$calculated for $\mathrm{C}_{16} \mathrm{H}_{21} \mathrm{~F}_{3} \mathrm{O}_{2}: 302.1494$, found 302.1485.

$[\alpha]_{\mathbf{D}}^{20.6}=+0.6^{\circ}\left(\mathrm{c} 1.32, \mathrm{CHCl}_{3}\right)$. 


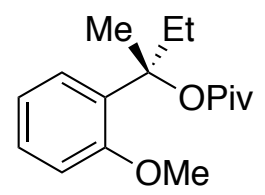

(S)-2-(2-methoxyphenyl)butan-2-yl pivalate (1f). Prepared via General Procedure B. The crude mixture was purified by silica gel chromatography $\left(2-20 \% \mathrm{Et}_{2} \mathrm{O} /\right.$ hexanes$)$ to give $\mathbf{1 f}$ ( $449.7 \mathrm{mg}$, $85 \%, 2.0 \mathrm{mmol}$ scale) as a colorless oil.

Enantiomeric Purity: 90\% ee by chiral HPLC analysis (CHIRALPAK OJ - H, $1.0 \mathrm{~mL} / \mathrm{min}, 2.0 \%$ $i$-PrOH/hexanes, $\lambda=220 \mathrm{~nm}$ ); $t_{\mathrm{R}}$ (major) $=6.175 \mathrm{~min}, t_{\mathrm{R}}($ minor $)=5.335 \mathrm{~min}$.

${ }^{1} \mathbf{H}$ NMR (600 MHz, $\left.\mathbf{C D C l}_{3}\right) \delta 7.31(\mathrm{dd}, J=7.8,1.7 \mathrm{~Hz}, 1 \mathrm{H}), 7.22(\mathrm{ddd}, J=8.2,7.3,1.7 \mathrm{~Hz}, 1 \mathrm{H})$, $6.92(\mathrm{td}, J=7.6,1.2 \mathrm{~Hz}, 1 \mathrm{H}), 6.87$ (dd, $J=8.2,1.2 \mathrm{~Hz}, 1 \mathrm{H}), 3.79$ (s, 3H), 2.21 (q, $J=7.4 \mathrm{~Hz}, 2 \mathrm{H})$, $1.85(\mathrm{~d}, J=1.6 \mathrm{~Hz}, 3 \mathrm{H}), 1.25(\mathrm{~d}, J=1.8 \mathrm{~Hz}, 9 \mathrm{H}), 0.75(\mathrm{t}, J=7.4 \mathrm{~Hz}, 3 \mathrm{H})$.

${ }^{13}$ C NMR (151 MHz, $\left.\mathbf{C D C l}_{3}\right) \delta 176.8,156.2,132.3,128.2,127.0,120.3,111.5,84.3,55.1,39.7$, $31.8,27.6,24.3,8.5$.

HRMS (EI+): [M] calculated for $\mathrm{C}_{16} \mathrm{H}_{24} \mathrm{O}_{3}: 264.1725$, found 264.1727 .

$[\alpha]_{\mathrm{D}}^{20.8}=-8.6^{\circ}\left(\mathrm{c} 1.09, \mathrm{CHCl}_{3}\right)$. 


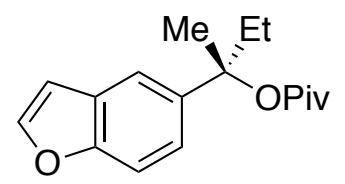

(S)-2-(benzofuran-5-yl)butan-2-yl pivalate (1h). Prepared via General Procedure B. The crude mixture was purified by silica gel chromatography $\left(2-20 \% \mathrm{Et}_{2} \mathrm{O} /\right.$ hexanes $)$ to give $\mathbf{1 h}(522.3 \mathrm{mg}$, $95 \%, 2.0 \mathrm{mmol}$ scale) as a colorless oil.

Enantiomeric Purity: 95\% ee by chiral HPLC analysis (CHIRALPAK IC, $1.0 \mathrm{~mL} / \mathrm{min}, 1.0 \% i$ PrOH/hexanes, $\lambda=254 \mathrm{~nm}) ; t_{\mathrm{R}}($ major $)=13.717 \mathrm{~min}, t_{\mathrm{R}}($ minor $)=9.321 \mathrm{~min}$.

${ }^{1}$ H NMR (600 MHz, $\left.\mathbf{C D C l}_{3}\right) \delta 7.60(\mathrm{~d}, J=2.2 \mathrm{~Hz}, 1 \mathrm{H}), 7.57-7.52(\mathrm{~m}, 1 \mathrm{H}), 7.46-7.42(\mathrm{~m}, 1 \mathrm{H})$, $7.28-7.23(\mathrm{~m}, 1 \mathrm{H}), 6.74(\mathrm{dd}, J=2.2,1.0 \mathrm{~Hz}, 1 \mathrm{H}), 2.10$ (q, $J=7.4 \mathrm{~Hz}, 2 \mathrm{H}), 1.86$ (s, 3H), 1.24 (s, $9 \mathrm{H}), 0.82(\mathrm{t}, J=7.4 \mathrm{~Hz}, 3 \mathrm{H})$.

${ }^{13}$ C NMR (151 MHz, CDCl $) \delta 177.0,154.0,145.4,140.1,127.3,121.3,117.4,111.1,107.0,84.0$, $39.5,35.7,27.4,24.8,8.4$.

HRMS (EI+): [M] calculated for $\mathrm{C}_{17} \mathrm{H}_{22} \mathrm{O}_{3}: 274.1569$, found 274.1570 .

$[\alpha]_{\mathrm{D}}^{21.1}=-2.1^{\circ}\left(\mathrm{c} 1.39, \mathrm{CHCl}_{3}\right)$. 


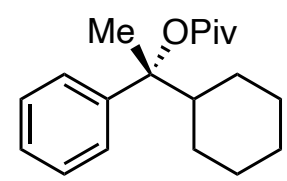

(R)-1-cyclohexyl-1-phenylethyl pivalate (1i). Prepared via General Procedure B (the reaction was allowed for overnight). The crude mixture was purified by silica gel chromatography ( $1-10 \%$ $\mathrm{Et}_{2} \mathrm{O} /$ hexanes) to give $\mathbf{1 i}(440.6 \mathrm{mg}, 72 \%$, 2-step) as a pale yellow oil.

Enantiomeric Purity: 95\% ee by chiral HPLC analysis (CHIRALPAK IC, $1.0 \mathrm{~mL} / \mathrm{min}, 1.0 \% i$ PrOH/hexanes, $\lambda=210 \mathrm{~nm}) ; t_{\mathrm{R}}($ major $)=6.121 \mathrm{~min}, t_{\mathrm{R}}($ minor $)=8.854 \mathrm{~min}$.

${ }^{1} \mathbf{H}$ NMR (600 MHz, $\left.\mathbf{C D C l}_{3}\right) \delta 7.31-7.27(\mathrm{~m}, 2 \mathrm{H}), 7.23-7.19(\mathrm{~m}, 3 \mathrm{H}), 1.90(\mathrm{dt}, J=12.8,3.2$ $\mathrm{Hz}, 1 \mathrm{H}), 1.82$ (s, 3H), $1.80-1.75$ (m, 1H), $1.75-1.60(\mathrm{~m}, 3 \mathrm{H}), 1.39-1.32(\mathrm{~m}, 1 \mathrm{H}), 1.26-1.16$ (m, 10H), $1.14-1.02(\mathrm{~m}, 3 \mathrm{H}), 0.97-0.88(\mathrm{~m}, 1 \mathrm{H})$.

${ }^{13} \mathbf{C}$ NMR (151 MHz, $\left.\mathbf{C D C l}_{3}\right) \delta$ 176.7, 144.4, 127.9, 126.7, 125.2, 85.5, 50.4, 39.6, 27.8, 27.4, $27.2,26.8,26.74,26.69,20.1$.

HRMS (LIFDI+): [M] ${ }^{+}$calculated for $\mathrm{C}_{19} \mathrm{H}_{28} \mathrm{O}_{2}: 288.2089$, found 288.2095.

$[\alpha]_{\mathbf{D}}^{21.0}=+9.3^{\circ}\left(\mathrm{c} 1.15, \mathrm{CHCl}_{3}\right)$. 


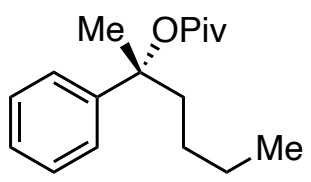

(R)-2-phenylhexan-2-yl pivalate (1j). Prepared via General Procedure B (the reaction was allowed for overnight). The crude mixture was purified by silica gel chromatography $(1-10 \%$ $\mathrm{Et}_{2} \mathrm{O} /$ hexanes) to give $\mathbf{1 j}$ ( $460.8 \mathrm{mg}, 49 \%$, 2-step) as a pale yellow oil.

Enantiomeric Purity: 96\% ee by chiral HPLC analysis (CHIRALPAK OJ - H, $1.0 \mathrm{~mL} / \mathrm{min}, 1.0 \%$ $i$-PrOH/hexanes, $\lambda=210 \mathrm{~nm}$ ); $t_{\mathrm{R}}$ (major) $=4.286 \mathrm{~min}, t_{\mathrm{R}}($ minor $)=4.853 \mathrm{~min}$.

${ }^{1}$ H NMR (600 MHz, $\left.\mathbf{C D C l}_{3}\right) \delta 7.34-7.29$ (m, 4H), $7.25-7.21$ (m, 1H), 2.04 (ddd, $J=13.7,10.5$, $6.0 \mathrm{~Hz}, 1 \mathrm{H}), 1.96$ (ddd, $J=13.7,10.5,5.8 \mathrm{~Hz}, 1 \mathrm{H}), 1.81(\mathrm{~s}, 3 \mathrm{H}), 1.23(\mathrm{~s}, 11 \mathrm{H}), 1.21-1.14(\mathrm{~m}$, $2 \mathrm{H}), 0.85(\mathrm{t}, \mathrm{J}=7.3 \mathrm{~Hz}, 3 \mathrm{H})$.

${ }^{13}$ C NMR (151 MHz, $\left.\mathbf{C D C l}_{3}\right) \delta$ 176.9, 145.7, 128.2, 126.8, 124.6, 83.5, 42.3, 39.5, 27.4, 26.0, $25.0,23.0,14.1$.

HRMS (LIFDI+): [M] ${ }^{+}$calculated for $\mathrm{C}_{17} \mathrm{H}_{26} \mathrm{O}_{2}: 262.1933$, found 262.1931 .

$[\alpha]_{\mathrm{D}}^{21.3}=-3.6^{\circ}\left(\mathrm{c} 1.46, \mathrm{CHCl}_{3}\right)$. 


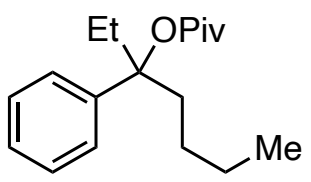

3-phenylheptan-3-yl pivalate (1k). Prepared via General Procedure B (the reaction was allowed for overnight, and LiHMDS was used in lieu of KHMDS). The crude mixture was purified by silica gel chromatography ( $2-20 \% \mathrm{Et}_{2} \mathrm{O} /$ hexanes) to give $1 \mathbf{k}(444.3 \mathrm{mg}, 80 \%, 2.0 \mathrm{mmol} \mathrm{scale})$ as a pale yellow oil.

${ }^{1} \mathbf{H}$ NMR (600 MHz, $\left.\mathbf{C D C l}_{3}\right) \delta 7.34-7.32(\mathrm{~m}, 4 \mathrm{H}), 7.25-7.21(\mathrm{~m}, 1 \mathrm{H}), 2.55-2.38(\mathrm{~m}, 2 \mathrm{H})$, $2.08-1.95(\mathrm{~m}, 2 \mathrm{H}), 1.28(\mathrm{~s}, 9 \mathrm{H}), 1.25-1.16(\mathrm{~m}, 2 \mathrm{H}), 1.11-1.02(\mathrm{~m}, 1 \mathrm{H}), 1.00-0.92(\mathrm{~m}, 1 \mathrm{H})$, 0.80 (t, $J=7.4 \mathrm{~Hz}, 3 \mathrm{H}), 0.66$ (t, $J=7.4 \mathrm{~Hz}, 3 \mathrm{H})$.

${ }^{13} \mathbf{C}$ NMR (151 MHz, $\left.\mathbf{C D C l}_{3}\right) \delta 176.8,144.2,128.1,126.7,125.2,87.4,39.9,37.7,30.9,27.7$, 25.5, 22.9, 14.1, 7.7.

HRMS (LIFDI+): [M] $]^{+}$calculated for $\mathrm{C}_{18} \mathrm{H}_{28} \mathrm{O}_{2}: 276.2089$, found 276.2090. 


\section{Preparation of Tertiary Benzylic Alcohols}

Tertiary benzylic alcohols $\mathbf{O H}-1, \mathbf{O H}-2, \mathbf{O H}-3, \mathbf{O H}-\mathbf{7}$ and $\mathbf{O H}-8$ were prepared as reported previously, ${ }^{4}$ as well as $\mathbf{O H}-11^{5}$.<smiles>CC[C@@](C)(O)c1ccccc1</smiles>

OH-1<smiles>CC[C@@](C)(O)c1ccc(F)cc1</smiles><smiles>CC[C@@]1(O)CCOc2ccccc21</smiles><smiles>CCCC[C@](C)(O)c1ccccc1</smiles><smiles>CC[C@@](C)(O)c1ccc(OC)cc1</smiles><smiles>CC[C@@](C)(O)c1ccc(C(F)(F)F)cc1</smiles><smiles>CC[C@@](C)(O)c1ccc2occc2c1</smiles><smiles>CCCCC(O)(CC)c1ccccc1</smiles><smiles>CC[C@@](C)(O)c1ccc2c(c1)OCO2</smiles><smiles>CC[C@@](C)(O)c1ccccc1OC</smiles><smiles>C[C@@](O)(c1ccccc1)C1CCCCC1</smiles> 


\section{General Procedure C: Preparation of Enantioenriched Tertiary Benzyl Alcohols}

Enantioenriched tertiary alcohols were prepared via the method used in our previous work. ${ }^{3}$ These procedures was adapted from the elegant method by Walsh and co-workers. ${ }^{6,7}$
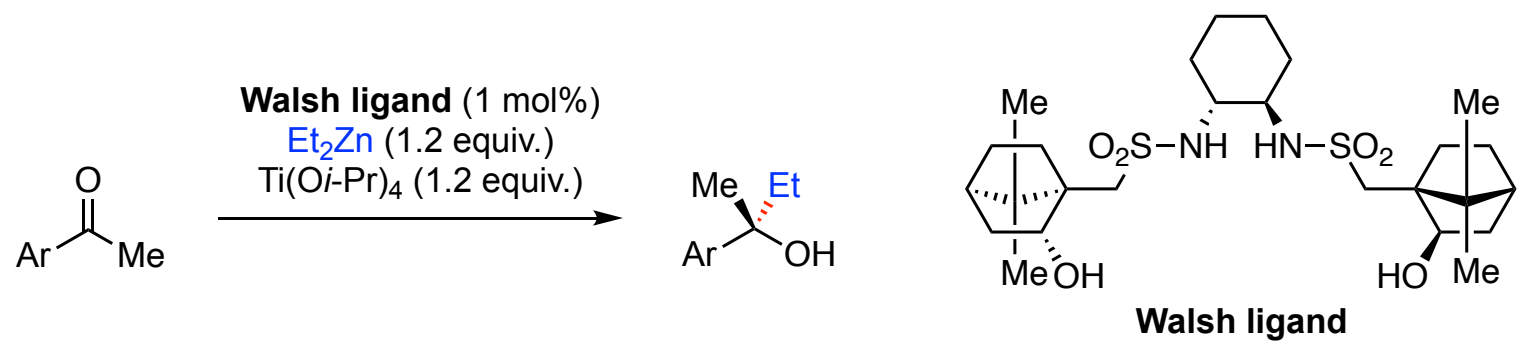

In an oven-dried, 100-mL, round-bottomed flask was placed Walsh ligand (33 mg, $0.060 \mathrm{mmol}$, 0.010 equiv) and $\mathrm{Et}_{2} \mathrm{Zn}(0.73 \mathrm{~mL}, 7.2 \mathrm{mmol}, 1.2 \text { equiv). Ti(Oi-Pr })_{4}(2.1 \mathrm{~mL}, 7.2 \mathrm{mmol}, 1.2$ equiv $)$ was added. The resulting solution was stirred at room temperature for $5 \mathrm{~min}$. Corresponding ketone (6.00 mmol, 1.00 equiv) was added into the flask in one portion. The mixture was stirred at room temperature for $17 \mathrm{~h}$. The resulting mixture was diluted with EtOAc (ca. $25 \mathrm{~mL}$ ) and quenched with sat. $\mathrm{NH}_{4} \mathrm{Cl}$. The solid was removed via filtration through a pad of silica gel. Rinsed with EtOAc (ca. $25 \mathrm{~mL})$. The product was extracted from the aqueous layer with EtOAc (30 $\mathrm{mL} \times 3)$. The combined organic layers were washed with sat. $\mathrm{NaCl}$, dried $\left(\mathrm{Na}_{2} \mathrm{SO}_{4}\right)$, filtered, and concentrated. The residue was purified via silica gel chromatography. 


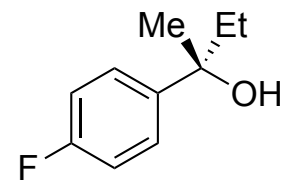

(S)-2-(4-fluorophenyl)butan-2-ol (OH-4). Prepared via General Procedure C. The crude mixture was purified by silica gel chromatography ( $2-20 \%$ EtOAc/hexanes) to give OH-4 (429.2 mg, $43 \%)$ as a colorless oil.

Enantiomeric Purity: 90\% ee by chiral HPLC analysis (CHIRALPAK OJ - H, $1.0 \mathrm{~mL} / \mathrm{min}, 3.0 \%$ $i-\mathrm{PrOH} / \mathrm{h}$ exanes, $\lambda=210 \mathrm{~nm}) ; t_{\mathrm{R}}($ major $)=11.113 \mathrm{~min}, t_{\mathrm{R}}($ minor $)=10.134 \mathrm{~min}$.

${ }^{1} \mathbf{H}$ NMR (600 MHz, $\left.\mathbf{C D C l}_{3}\right) \delta 7.42-7.37(\mathrm{~m}, 2 \mathrm{H}), 7.05-6.98(\mathrm{~m}, 2 \mathrm{H}), 1.89-1.75(\mathrm{~m}, 2 \mathrm{H})$, $1.65(\mathrm{~s}, 1 \mathrm{H}), 1.54$ (s, 3H), 0.79 (t, $J=7.5 \mathrm{~Hz}, 3 \mathrm{H})$.

${ }^{13} \mathrm{C}$ NMR (151 MHz, CDCl $) \delta 161.8\left(\mathrm{~d}, J_{\mathrm{C}-\mathrm{F}}=244.6 \mathrm{~Hz}\right), 143.6\left(\mathrm{~d}, J_{\mathrm{C}-\mathrm{F}}=3.2 \mathrm{~Hz}\right), 126.8\left(\mathrm{~d}, J_{\mathrm{C}-}\right.$ $\mathrm{F}=7.9 \mathrm{~Hz}), 114.9\left(\mathrm{~d}, J_{\mathrm{C}-\mathrm{F}}=21.1 \mathrm{~Hz}\right), 74.8,37.0,29.9,8.4$.

${ }^{19}$ F NMR (565 MHz, $\left.\mathrm{CDCl}_{3}\right) \delta-117.1$.

HRMS (LIFDI+): [M] $]^{+}$calculated for $\mathrm{C}_{10} \mathrm{H}_{13} \mathrm{FO}: 168.0950$, found 168.0947 .

$[\alpha]_{\mathbf{D}}^{2.33}=-12.7^{\circ}\left(\mathrm{c} 1.45, \mathrm{CHCl}_{3}\right)$.

The spectral data matches that of the literature. ${ }^{8}$ 


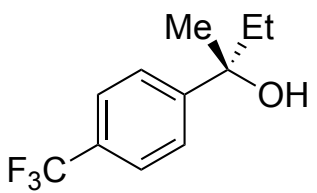

(S)-2-(4-(trifluoromethyl)phenyl)butan-2-ol (OH-5). Prepared via General Procedure C. The crude mixture was purified by silica gel chromatography $(2-20 \%$ EtOAc/hexanes $)$ to give $\mathbf{O H}-5$ (487.6 $\mathrm{mg}, 37 \%)$ as a pale yellow oil.

Enantiomeric Purity: 89\% ee by chiral HPLC analysis (CHIRALPAK OJ - H, $1.0 \mathrm{~mL} / \mathrm{min}, 3.0 \%$ $i-\mathrm{PrOH} /$ hexanes, $\lambda=220 \mathrm{~nm}) ; t_{\mathrm{R}}$ (major) $=7.846 \mathrm{~min}, t_{\mathrm{R}}($ minor $)=7.134 \mathrm{~min}$.

${ }^{1}$ H NMR (600 MHz, CDCl $) \delta 7.61-7.53$ (m, 4H), 1.93 - 1.77 (m, 2H), 1.74 (s, 1H), 1.56 (s, $3 \mathrm{H}), 0.80(\mathrm{t}, J=7.4 \mathrm{~Hz}, 3 \mathrm{H})$.

${ }^{13}$ C NMR (151 MHz, CDCl $) \delta 151.9,129.0\left(\mathrm{q}, J_{\mathrm{C}-\mathrm{F}}=32.4 \mathrm{~Hz}\right), 125.5,125.2\left(\mathrm{q}, J_{\mathrm{C}-\mathrm{F}}=3.8 \mathrm{~Hz}\right)$, $124.4\left(\mathrm{q}, J_{\mathrm{C}-\mathrm{F}}=271.8 \mathrm{~Hz}\right), 75.0,36.8,30.0,8.2$.

${ }^{19}$ F NMR (565 MHz, CDCl 3$) \delta-62.4$.

HRMS (LIFDI+): [M] $]^{+}$calculated for $\mathrm{C}_{11} \mathrm{H}_{13} \mathrm{~F}_{3} \mathrm{O}: 218.0918$, found 218.0907 .

$[\alpha]_{\mathrm{D}}^{23.6}=-10.1^{\circ}\left(\mathrm{c} 1.08, \mathrm{CHCl}_{3}\right)$.

The spectral data matches that of the literature. ${ }^{9}$ 


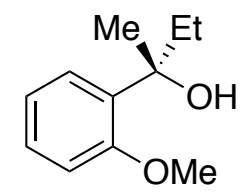

(S)-2-(2-methoxyphenyl)butan-2-ol (OH-6). Prepared via General Procedure C. The crude mixture was purified by silica gel chromatography $\left(2-20 \% \mathrm{Et}_{2} \mathrm{O} /\right.$ hexanes) to give $\mathbf{O H}-6$ (479.6 $\mathrm{mg}, 44 \%)$ as a colorless oil.

Enantiomeric Purity: 90\% ee by chiral HPLC analysis (CHIRALPAK IB, $1.0 \mathrm{~mL} / \mathrm{min}, 1.0 \% i$ $\mathrm{PrOH} / \mathrm{h}$ exanes, $\lambda=220 \mathrm{~nm}) ; t_{\mathrm{R}}($ major $)=13.026 \mathrm{~min}, t_{\mathrm{R}}($ minor $)=10.949 \mathrm{~min}$.

${ }^{1} \mathbf{H}$ NMR $\left(600 \mathrm{MHz}, \mathbf{C D C l}_{3}\right) \delta 7.28(\mathrm{dd}, J=7.7,1.7 \mathrm{~Hz}, 1 \mathrm{H}), 7.23(\mathrm{ddd}, J=8.2,7.4,1.7 \mathrm{~Hz}, 1 \mathrm{H})$, $6.99-6.88(\mathrm{~m}, 2 \mathrm{H}), 4.04$ (s, 1H), 3.89 (s, 3H), $2.01-1.84(\mathrm{~m}, 2 \mathrm{H}), 1.56(\mathrm{~s}, 3 \mathrm{H}), 0.81$ (t, $J=7.5$ $\mathrm{Hz}, 3 \mathrm{H})$.

${ }^{13}$ C NMR (151 MHz, $\left.\mathbf{C D C l}_{3}\right) \delta 157.1,134.9,128.1,127.1,121.0,111.5,75.6,55.5,34.9,26.8$, 9.0.

HRMS (LIFDI+): $[\mathrm{M}]^{+}$calculated for $\mathrm{C}_{11} \mathrm{H}_{16} \mathrm{O}_{2}: 180.1150$, found 180.1155 .

$[\alpha]_{\mathrm{D}}^{23.8}=+1.7^{\circ}\left(\mathrm{c} 1.35, \mathrm{CHCl}_{3}\right)$.

The spectral data matches that of the literature. ${ }^{10}$ 
Enantioenriched tertiary alcohols $\mathbf{O H}-\mathbf{9}$ and $\mathbf{O H}-10$ were prepared according to Aggarwal's work ("One-Pot Preparation of Chiral Tertiary Alcohols" method). ${ }^{11}$

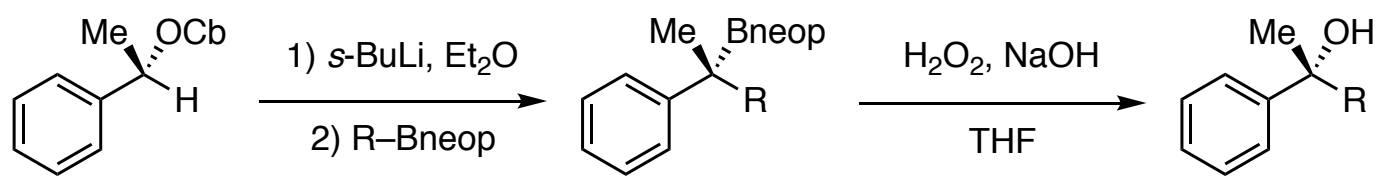

After work-up, the residue was purified by silica gel chromatography (EtOAc/hexanes) to give a colorless oil, which was used directly in the next step.

\section{Mechanistic Experiments}

Note: $\mathrm{PhMe}(0.1 \mathrm{M})$ was used in our mechanistic experiments to provide greater solubility and prevent solvent loss with removal of aliquots. Although PhMe was not optimal in our optimization studies, it does provide similar yields and stereochemical fidelity.<smiles>CCC(C)(c1ccccc1)c1cc(OC)c(OC)c(OC)c1</smiles>

2-(3,4,5-Trimethoxyphenylbutan-2-yl)benzene (24). Product 24 was prepared via General Procedure A, except that $\mathrm{PhMe}(4.0 \mathrm{~mL}, 0.1 \mathrm{M})$ was used in place of $\mathrm{CyH}$ and the reaction was performed in a 2-dram vial. The crude mixture was purified by silica gel chromatography (1-3\% EtOAc/PhMe) to give $\mathbf{2 4}(101 \mathrm{mg}, 84 \%)$ as a colorless oil.

${ }^{1} \mathbf{H}$ NMR (400 MHz, $\left.\mathbf{C D C l}_{3}\right) \delta 7.30$ - 7.24 (m, 2H), $7.23-7.14$ (m, 3H), 6.39 (s, 2H), 3.84 (s, $3 \mathrm{H}), 3.76$ (s, 6H), 2.11 (qd, $J=7.1,4.1 \mathrm{~Hz}, 2 \mathrm{H}), 1.59$ (s, 3H), 0.75 (t, $J=7.3 \mathrm{~Hz}, 3 \mathrm{H})$.

${ }^{13}$ C NMR (101 MHz, CDCl $) \delta$ 152.6, 149.5, 145.6, 136.0, 128.0, 127.5, 125.7, 105.1, 61.0, $56.2,46.9,34.3,27.2,9.4$.

FTIR (thin film): 2966, 2932, 1587, 1512, 1462, 1411, 1332, 1243, 1126, 1011, $702 \mathrm{~cm}^{-1}$. HRMS (ESI+): $[\mathrm{M}+\mathrm{H}]^{+}$calculated for $\mathrm{C}_{19} \mathrm{H}_{2} \mathrm{O}_{3}: 301.1804$, found 301.1813 . 


\section{Reaction Progress Analysis}<smiles>CCC(C)(O[Na])c1ccccc1</smiles>

$( \pm)-1 a$<smiles>COc1cc(P)cc(OC)c1OC</smiles>

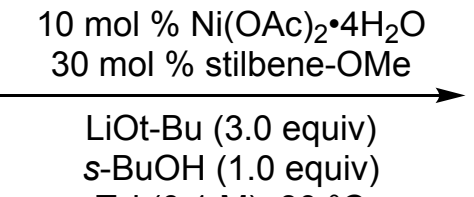

Tol $(0.1 \mathrm{M}), 80^{\circ} \mathrm{C}$

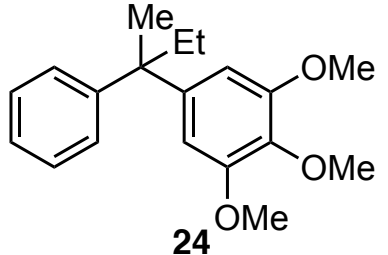

24

In a $\mathrm{N}_{2}$-filled glovebox, a 2-dram vial with a stirbar was charged with $\mathrm{Ni}(\mathrm{OAc})_{2} \cdot 4 \mathrm{H}_{2} \mathrm{O}(10$ mol \%, $0.030 \mathrm{mmol}, 7.5 \mathrm{mg})$, stilbene-OMe (30 mol \%, $0.090 \mathrm{mmol}, 27 \mathrm{mg})$ and $\mathrm{LiO} t-\mathrm{Bu}(3.0$ equiv, $0.90 \mathrm{mmol}, 72 \mathrm{mg})$. 3,4,5-Trimethoxyphenylboronic acid pinacol ester (3.0 mmol, $882 \mathrm{mg})$ was weighed into a 5-mL volumetric flask, and $\mathrm{PhMe}$ was added to bring the total volume to 5.0 mL. $1.0 \mathrm{~mL}$ of this solution was added to the 2-dram vial, resulting in the addition of arylboronic ester (2.0 equiv, $0.60 \mathrm{mmol}, 176 \mathrm{mg})$. Pivalate (1.5 mmol, $352 \mathrm{mg})$, 1,3,5-trimethoxybenzene (internal standard, $1.5 \mathrm{mmol}, 252 \mathrm{mg})$, and $s-\mathrm{BuOH}(1.5 \mathrm{mmol}, 111 \mathrm{mg})$ were weighed into a second 5-mL volumetric flask, and PhMe was added to bring the total volume to $5 \mathrm{~mL}$. $1.0 \mathrm{~mL}$ of this second solution was transferred to the 2 -dram vial, resulting in the addition of pivalate (1.0 equiv, $0.3 \mathrm{mmol}, 71 \mathrm{mg}$ ), 1,3,5-trimethoxybenzene (internal standard, 1.0 equiv, $0.30 \mathrm{mmol}, 51$ $\mathrm{mg})$, and $s-\mathrm{BuOH}(1.0$ equiv, $0.30 \mathrm{mmol}, 22 \mathrm{mg})$. PhMe $(1.0 \mathrm{~mL})$ was then also added to the 2dram vial to result in $3.0 \mathrm{~mL}$ total $\mathrm{PhMe}$. The vial was capped with a Teflon pierceable cap and removed from glovebox. The vial was placed in a pre-heated $\left(80^{\circ} \mathrm{C}\right)$ hot plate. Aliquots of the reaction mixture $(0.1 \mathrm{~mL})$ were removed periodically and filtered through Kimwipe $\circledR$ plugs into $\mathrm{GC}$ vials. The filter was washed with $\mathrm{MeOH}$ to fill the $\mathrm{GC}$ vial. The concentration of remaining pivalate 1a and product $\mathbf{2 4}$ were then determined by GC analysis using 1,3,5-trimethoxybenzene as internal standard. Alterations to this procedure are noted in the data below. 

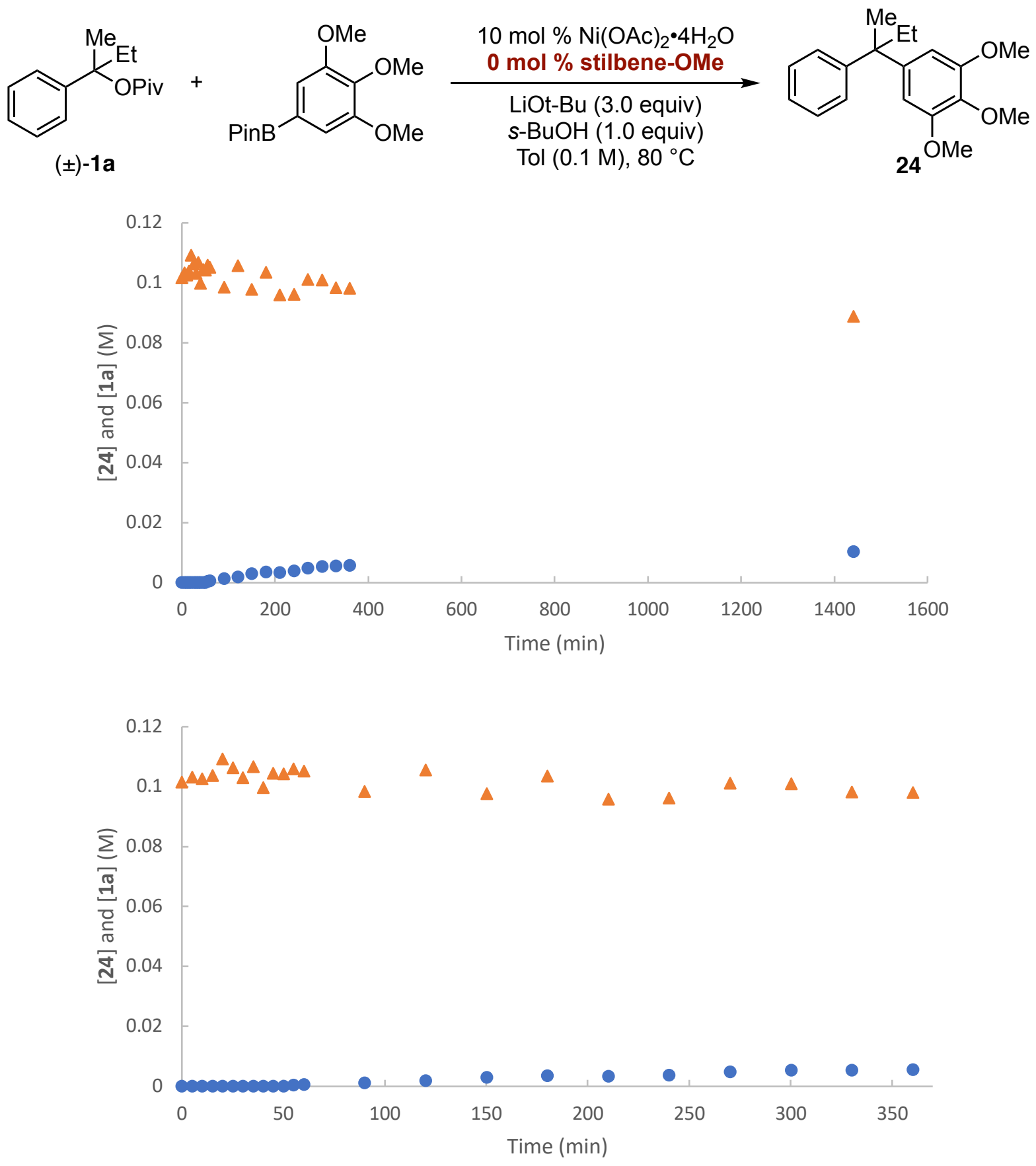

Figure S1. Reaction progress in the absence of L1 (0 mol \% L1). Top: 24 h. Bottom: Zoomed in to first $6 \mathrm{~h} . \bullet[\mathbf{2 4}] . \Delta[\mathbf{1 a}]$. 
<smiles>CCC(C)(O[Na])c1ccccc1</smiles>

$( \pm)-1 a$<smiles>COc1cc(BP)cc(OC)c1OC</smiles>

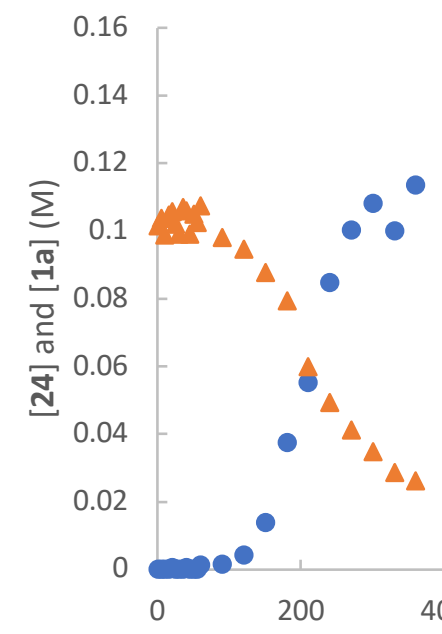

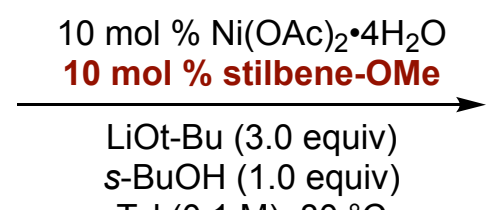

Tol (0.1 M), $80^{\circ} \mathrm{C}$

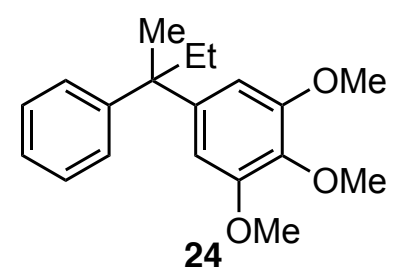

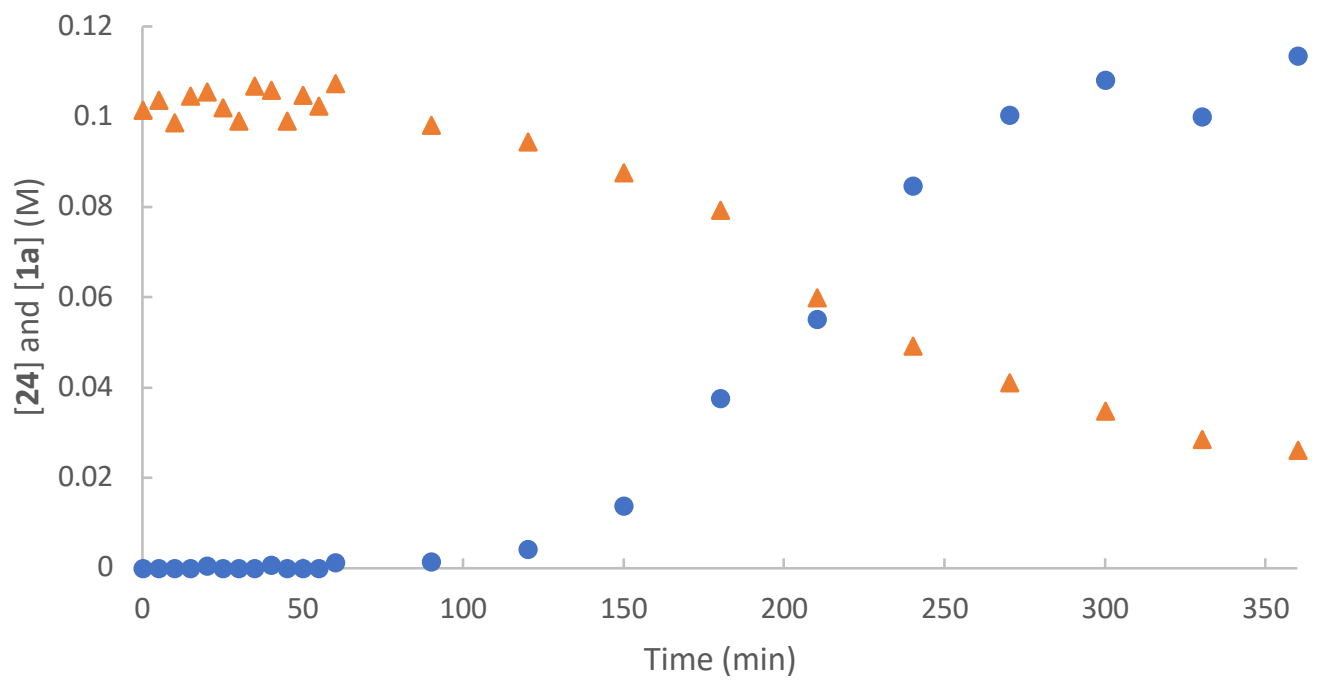

Figure S2. Reaction progress in the presence of $10 \mathrm{~mol} \% \mathbf{L 1}$. Top: $24 \mathrm{~h}$. Bottom: Zoomed in to first 6 h. $\bullet[\mathbf{2 4}] . \triangle[\mathbf{1 a}]$. 
<smiles>[M]C(CC)(O[NH3+])c1ccccc1</smiles>

$( \pm)-1 a$<smiles>COc1cc(P)cc(OC)c1OC</smiles>

$\mathrm{PinB} O \mathrm{OMe}$

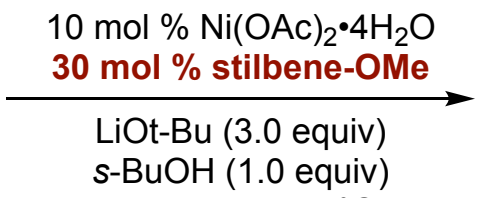

Tol $(0.1 \mathrm{M}), 80^{\circ} \mathrm{C}$

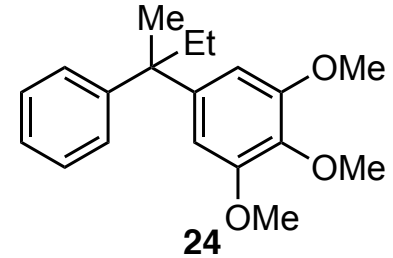

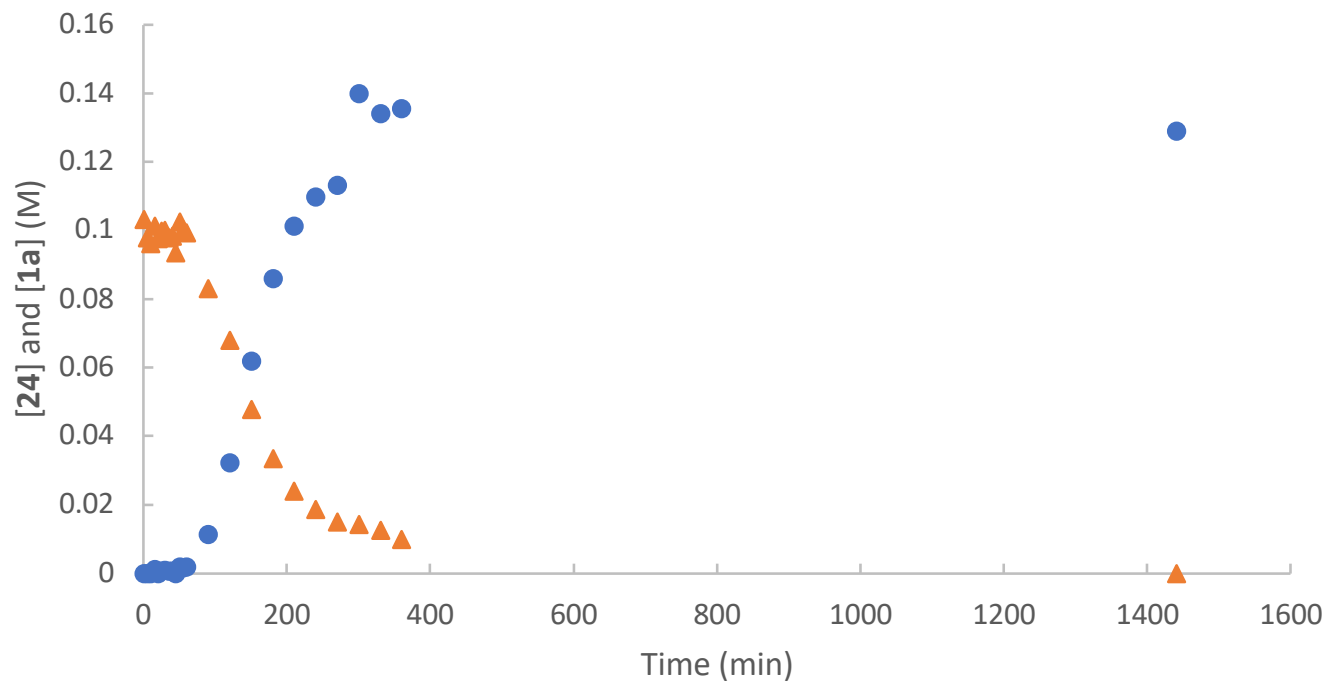

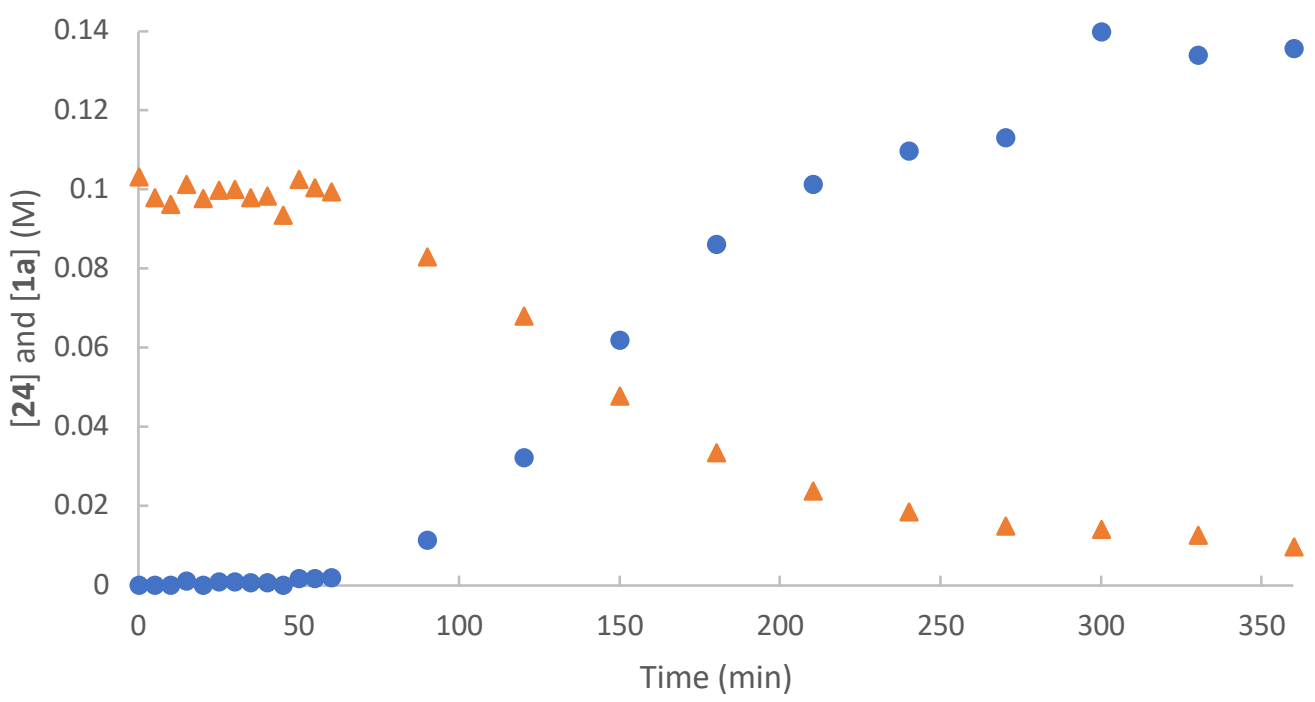

Figure S3. Reaction progress in the presence of $30 \mathrm{~mol} \%$ L1. Top: $24 \mathrm{~h}$. Bottom: Zoomed in to first $6 \mathrm{~h} . \bullet[\mathbf{2 4}] . \triangle[1 \mathbf{a}]$. 
<smiles>[M]C(CC)(O[NH3+])c1ccccc1</smiles>

$( \pm)-1 a$<smiles>COc1cc(BP)cc(OC)c1OC</smiles>

0.14

0.12
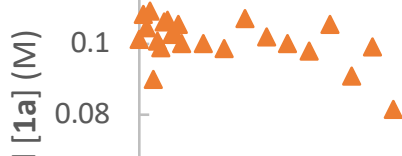

豪 0.06

志 0.04

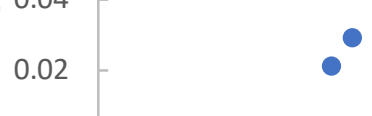

0

$0 \quad 200$

400

$10 \mathrm{~mol} \% \mathrm{Ni}(\mathrm{OAc})_{2} \cdot 4 \mathrm{H}_{2} \mathrm{O}$ $100 \mathrm{~mol} \%$ stilbene-OMe LiOt-Bu (3.0 equiv) $s-\mathrm{BuOH}$ (1.0 equiv) Tol (0.1 M), $80^{\circ} \mathrm{C}$<smiles>CCC(C)(c1ccccc1)c1cc(OC)c(OC)c(OC)c1</smiles>
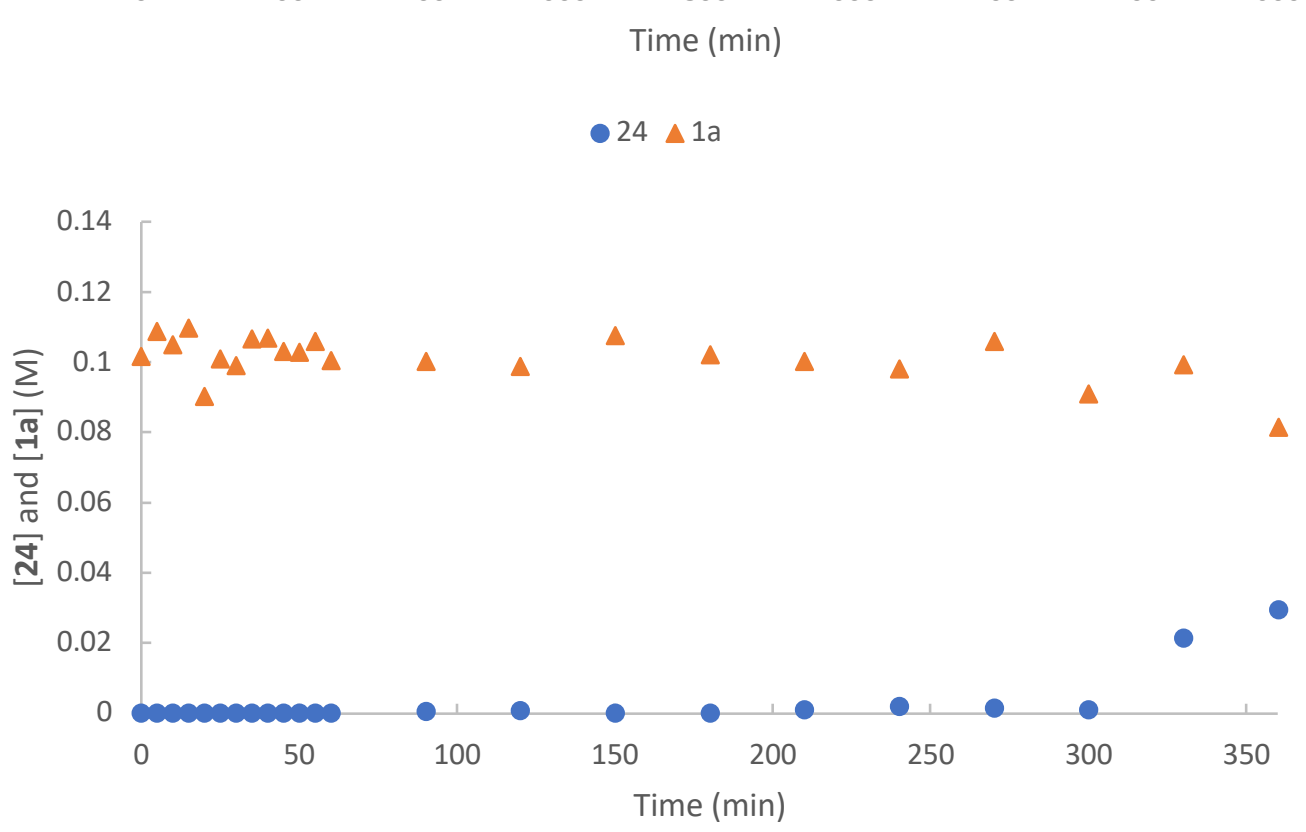

- $24 \Delta 1 a$

Figure S4. Reaction progress in the presence of 100 mol \% L1. Top: 24 h. Bottom: Zoomed in to first 6 h. $\bullet[\mathbf{2 4}] . \Delta[\mathbf{1 a}]$. 
<smiles>[M]C(CC)(O[N+])c1ccccc1</smiles>

$( \pm)-1 a$<smiles>[GeH2]</smiles>

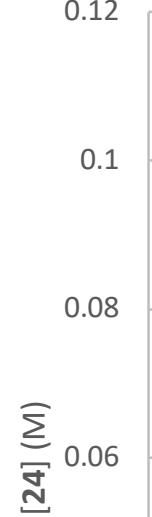

0.04

0.02

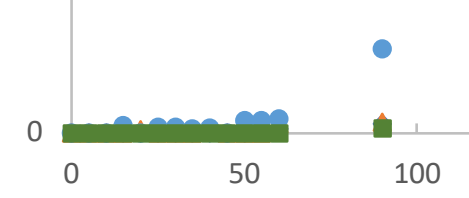<smiles>COc1cc(P)cc(OC)c1OC</smiles>

PinB OMe

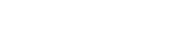

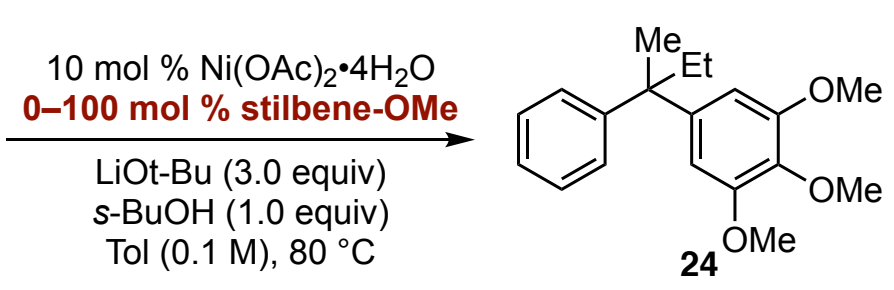

Tol $(0.1 \mathrm{M}), 80^{\circ} \mathrm{C}$

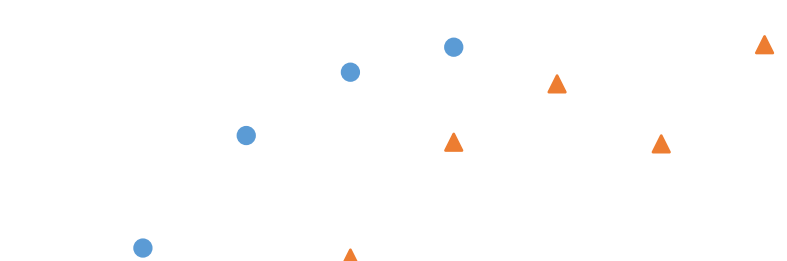

$\Delta$

$\Delta$

$\Delta$

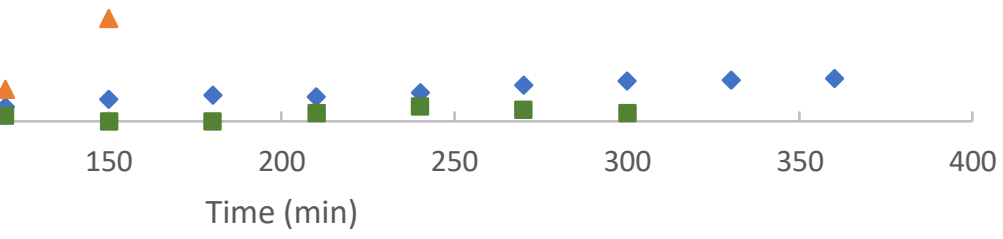

Figure S5. Comparison of reaction progress in the presence of varying amounts of $\mathbf{L 1}$ (combination of data from Figures S1-S4). 0 mol \% L1. $\triangle 10 \mathrm{~mol} \% \mathbf{L 1} . \bullet 30 \mathrm{~mol} \% \mathbf{L 1}$. $100 \mathrm{~mol} \mathrm{\%} \mathrm{L1.}$ 

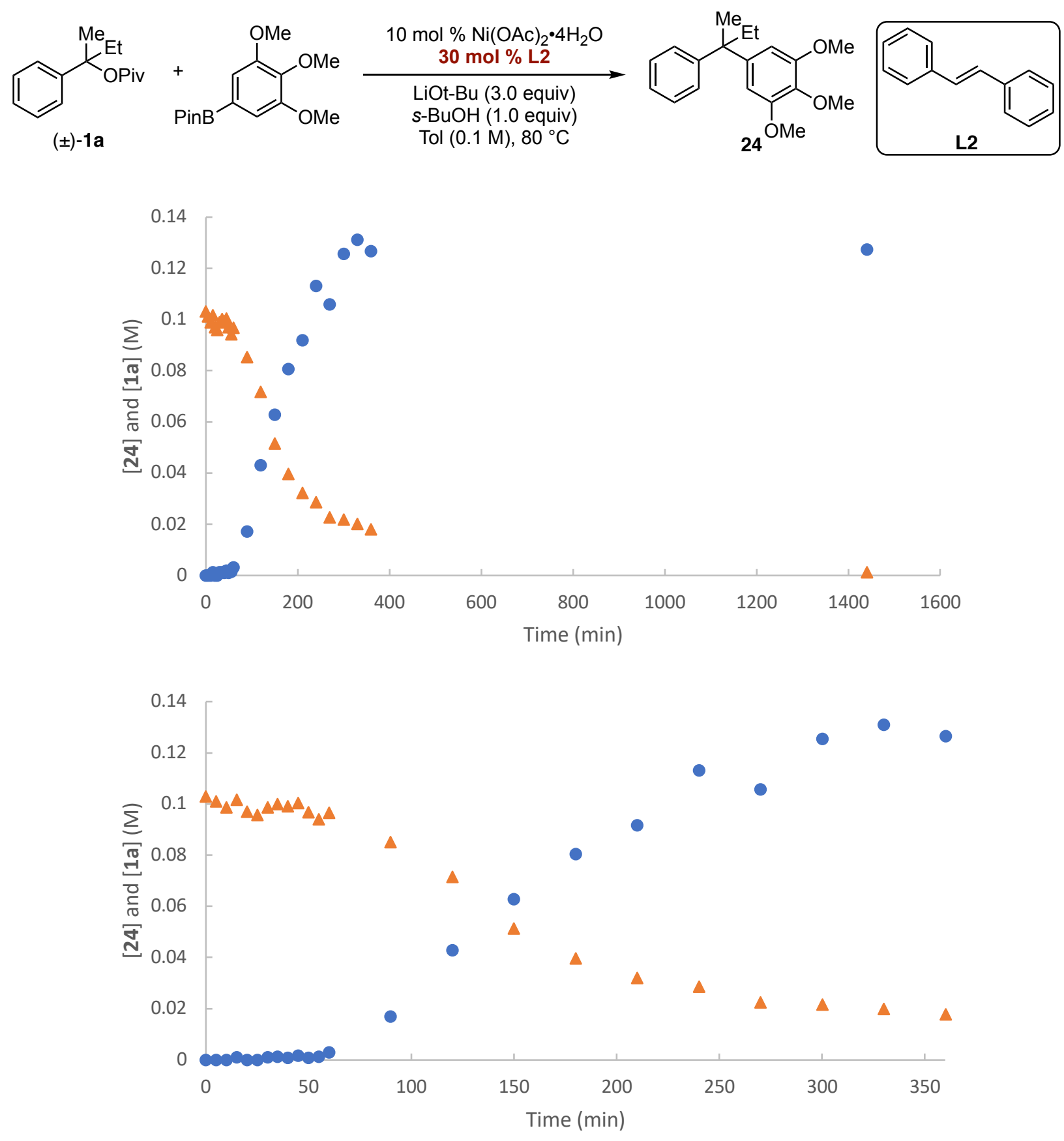

Figure S6. Reaction progress in the presence of $30 \mathrm{~mol} \% \mathbf{L 2}$ (stilbene). Top: $24 \mathrm{~h}$. Bottom: Zoomed in to first $6 \mathrm{~h} . \bullet[\mathbf{2 4}] . \Delta[\mathbf{1 a}]$. 
<smiles>CCC(C)(O[Na])c1ccccc1</smiles>

( \pm -1a<smiles>COc1cc(BP)cc(OC)c1OC</smiles>

PinB OMe

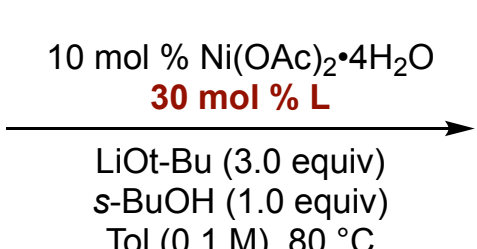

Tol $(0.1 \mathrm{M}), 80^{\circ} \mathrm{C}$

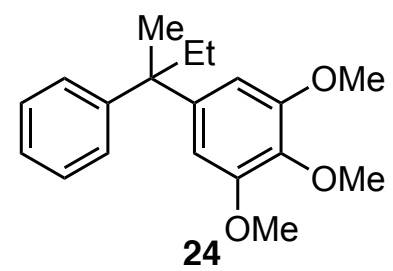<smiles>COc1cc(/C=C/c2cc(OC)cc(OC)c2)cc(OC)c1</smiles>

L1<smiles>C(=C/c1ccccc1)\c1ccccc1</smiles>

L2

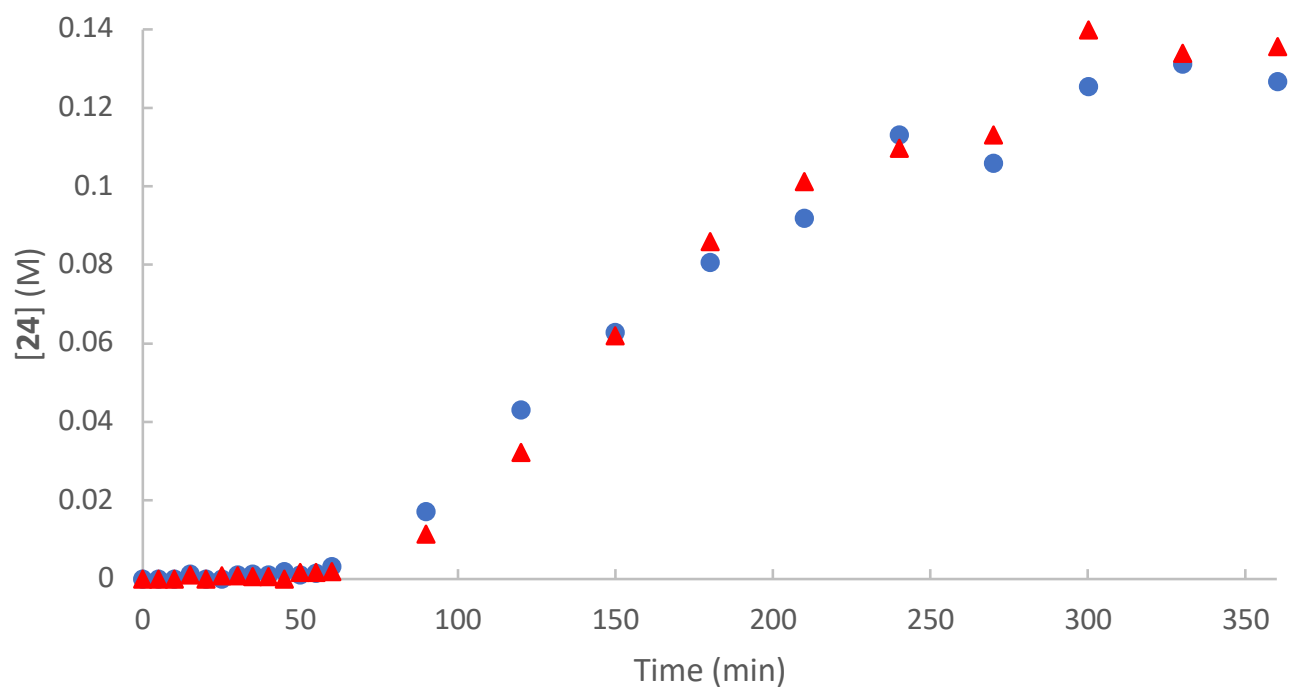

Figure S7. Comparison of reaction progress in the presence of $\mathbf{L} 1$ and $\mathbf{L} 2$ (combination of data from Figures S3 and S6). ^ $30 \mathrm{~mol} \%$ L1. $30 \mathrm{~mol} \% \mathbf{L 2}$. 


\section{Efforts to Eliminate the Induction Period}

We considered the possibility that the induction period may be caused by slow reduction of the $\mathrm{Ni}(\mathrm{OAc})_{2} \cdot 4 \mathrm{H}_{2} \mathrm{O}$ to $\mathrm{Ni}(0)$. To address this possibility, we performed an experiment in which MeLi was added to the $\mathrm{Ni}(\mathrm{OAc})_{2} \cdot 4 \mathrm{H}_{2} \mathrm{O}$ and $\mathbf{~} \mathbf{1}$ before the rest of the components were added. However, as shown below, this experiment still suffered from the induction period.<smiles>CCC(C)(O[N+])c1ccccc1</smiles>

$( \pm)-1 \mathrm{a}$<smiles>COc1cc(B=P)cc(OC)c1OC</smiles>

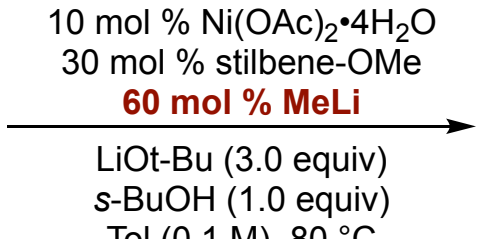

Tol $(0.1 \mathrm{M}), 80^{\circ} \mathrm{C}$<smiles>CCC(C)(c1ccccc1)c1cc(OC)c(OC)c(OC(C)=O)c1</smiles>

24

In a $\mathrm{N}_{2}$-filled glovebox, a 2-dram vial with a stirbar was charged with $\mathrm{Ni}(\mathrm{OAc})_{2} \cdot 4 \mathrm{H}_{2} \mathrm{O}(10$ $\mathrm{mol} \%, 0.030 \mathrm{mmol}, 7.5 \mathrm{mg})$, stilbene-OMe (30 mol \%, $0.090 \mathrm{mmol}, 27 \mathrm{mg})$, and PhMe (1.0 mL). $\mathrm{MeLi}\left(1.6 \mathrm{M}\right.$ in $\mathrm{Et}_{2} \mathrm{O}, 0.60$ equiv, $\left.0.18 \mathrm{mmol}, 112 \mu \mathrm{L}\right)$ was added. The solution was stirred at room temperature for $30 \mathrm{~min}$. $\mathrm{LiO} t-\mathrm{Bu}(3.0$ equiv, $0.90 \mathrm{mmol}, 72 \mathrm{mg})$ was added. 3,4,5Trimethoxyphenylboronic acid pinacol ester $(1.2 \mathrm{mmol}, 353 \mathrm{mg})$ was weighed into a 2-mL volumetric flask, and $\mathrm{PhMe}$ was added to bring the total volume to $2.0 \mathrm{~mL}$. $1.0 \mathrm{~mL}$ of this solution was added to the 2-dram vial, resulting in the addition of arylboronic ester ( 2.0 equiv, $0.60 \mathrm{mmol}$, $176 \mathrm{mg}$ ). Pivalate (0.60 mmol, $141 \mathrm{mg})$, 1,3,5-trimethoxybenzene (internal standard, $0.60 \mathrm{mmol}$, $101 \mathrm{mg}$ ), and $s-\mathrm{BuOH}(0.60 \mathrm{mmol}, 44 \mathrm{mg})$ were weighed into a second 2-mL volumetric flask, and $\mathrm{PhMe}$ was added to bring the total volume to $2 \mathrm{~mL} .1 .0 \mathrm{~mL}$ of this second solution was transferred to the 2-dram vial, resulting in the addition of pivalate (1.0 equiv, $0.30 \mathrm{mmol}, 71 \mathrm{mg}$ ), 1,3,5-trimethoxybenzene (internal standard, 1.0 equiv, $0.30 \mathrm{mmol}, 51 \mathrm{mg}$ ), and $s$ - $\mathrm{BuOH}(1.0$ equiv, $0.30 \mathrm{mmol}, 22 \mathrm{mg}$ ). The vial was capped with a Teflon pierceable cap and removed from glovebox. The vial was placed in a pre-heated $\left(80^{\circ} \mathrm{C}\right)$ hot plate. Aliquots of the reaction mixture $(0.1 \mathrm{~mL})$ were removed periodically and filtered through Kimwipe ${ }^{\circledR}$ plugs into GC vials. The filter was washed with $\mathrm{MeOH}$ to fill the $\mathrm{GC}$ vial. The concentration of remaining pivalate $\mathbf{1 a}$ and product $\mathbf{2 4}$ were then determined by GC analysis using 1,3,5-trimethoxybenzene as internal standard. 


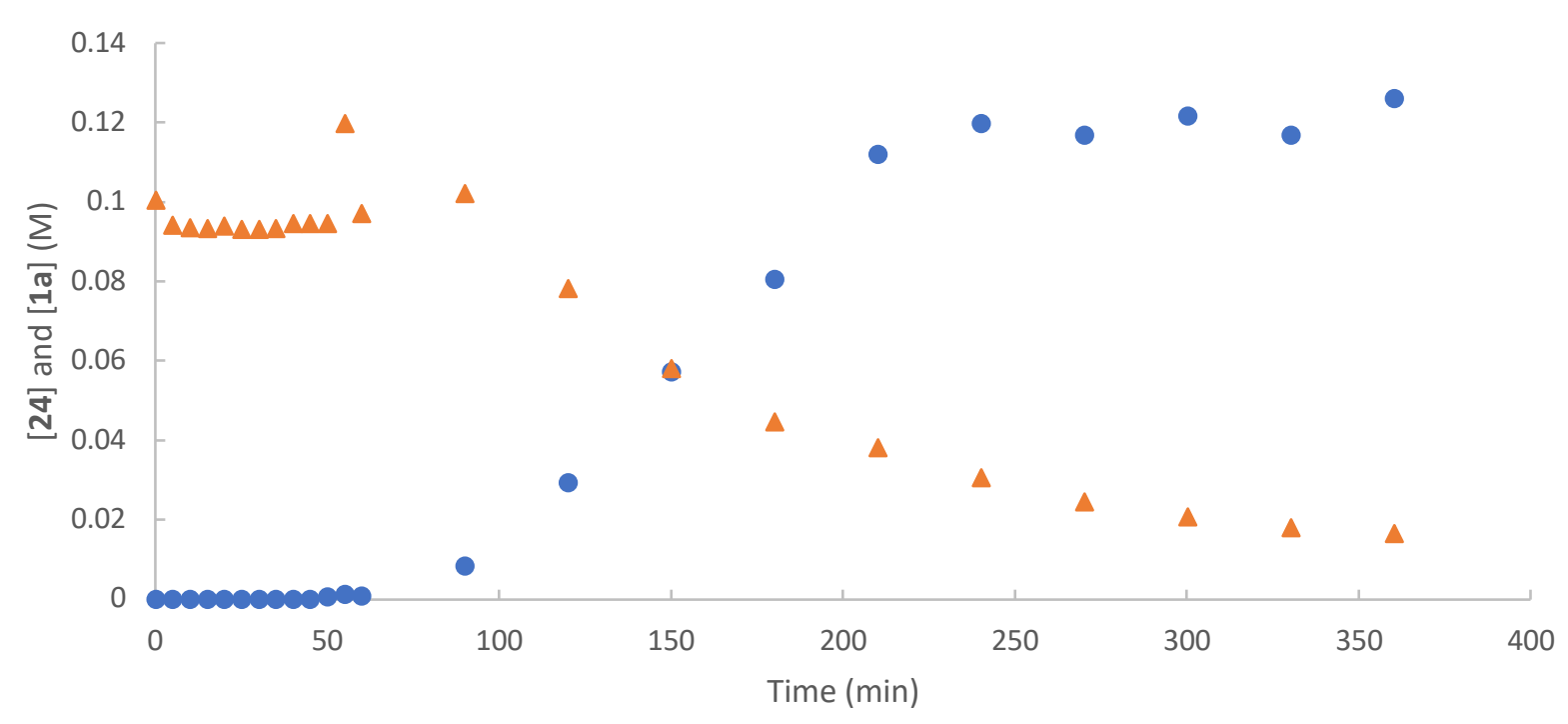

Figure S8. Reaction progress after reduction by MeLi. $\bullet[24] . \Delta[\mathbf{1 a}]$.

We also considered the possibility that the formation of the boronate complex may be contributing to the induction period and conducted a series of experiments to see if combination of the arylboronic ester and $\mathrm{LiO} t$-Bu before addition of the other components would eliminate the induction period. As shown below, although we observed changes to the induction period, the induction still persisted.<smiles>CCC(C)(O[N+])c1ccccc1</smiles>

$( \pm)-1 a$<smiles>COc1cc(P)cc(OC)c1OC</smiles>

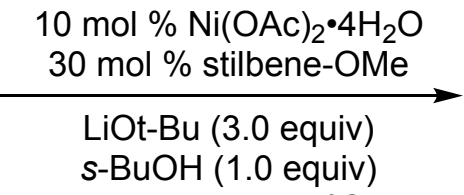

Tol $(0.1 \mathrm{M}), 80^{\circ} \mathrm{C}$ pre-mixing of LiOt-Bu + ArBPin<smiles>CCC(C)(c1ccccc1)c1cc(OC)c(OC)c(OC)c1</smiles>

In a $\mathrm{N}_{2}$-filled glovebox, a 2-dram vial with a stirbar was charged with $\mathrm{LiO} t$-Bu (3.0 equiv, $0.90 \mathrm{mmol}, 72 \mathrm{mg}$ ). In one case (see below), $s-\mathrm{BuOH}$ (1.0 equiv, $0.30 \mathrm{mmol}, 22 \mathrm{mg}$ ) was also added; in the other cases, $s$-BuOH was added later as described below. 3,4,5Trimethoxyphenylboronic acid pinacol ester $(3.0 \mathrm{mmol}, 882 \mathrm{mg})$ was weighed into a 5-mL volumetric flask, and $\mathrm{PhMe}$ was added to bring the total volume to $5.0 \mathrm{~mL}$. $1.0 \mathrm{~mL}$ of this solution was added to the 2-dram vial, resulting in the addition of arylboronic ester ( 2.0 equiv, $0.60 \mathrm{mmol}$, $176 \mathrm{mg}$ ). This mixture was stirred at the temperature and for the time noted below. Then, $\mathrm{Ni}(\mathrm{OAc})_{2} \cdot 4 \mathrm{H}_{2} \mathrm{O}(10 \mathrm{~mol} \%, 0.030 \mathrm{mmol}, 7.5 \mathrm{mg})$ and stilbene-OMe (30 mol \%, $0.090 \mathrm{mmol}, 27$ mg) were added. Pivalate (1.5 mmol, $352 \mathrm{mg})$, 1,3,5-trimethoxybenzene (internal standard, 1.5 
mmol, $252 \mathrm{mg}$ ), and $s-\mathrm{BuOH}$ if it was not previously added $(1.5 \mathrm{mmol}, 111 \mathrm{mg})$ were weighed into a second 5-mL volumetric flask, and PhMe was added to bring the total volume to $5 \mathrm{~mL}$. 1.0 $\mathrm{mL}$ of this second solution was transferred to the 2-dram vial, resulting in the addition of pivalate (1.0 equiv, $0.30 \mathrm{mmol}, 71 \mathrm{mg}$ ), 1,3,5-trimethoxybenzene (internal standard, 1.0 equiv, $0.30 \mathrm{mmol}$, $51 \mathrm{mg})$, and $s-\mathrm{BuOH}$ (1.0 equiv, $0.30 \mathrm{mmol}, 22 \mathrm{mg})$. PhMe $(1.0 \mathrm{~mL})$ was then also added to the 2-dram vial to result in $3.0 \mathrm{~mL}$ total PhMe. The vial was capped with a Teflon pierceable cap and removed from glovebox. The vial was placed in a pre-heated $\left(80^{\circ} \mathrm{C}\right)$ hot plate. Aliquots of the

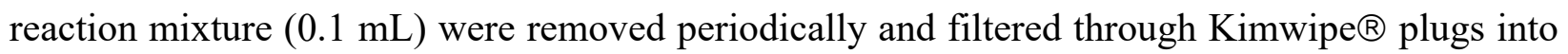
$\mathrm{GC}$ vials. The filter was washed with $\mathrm{MeOH}$ to fill the $\mathrm{GC}$ vial. The concentration of remaining pivalate 1a and product $\mathbf{2 4}$ were then determined by GC analysis using 1,3,5-trimethoxybenzene as internal standard.

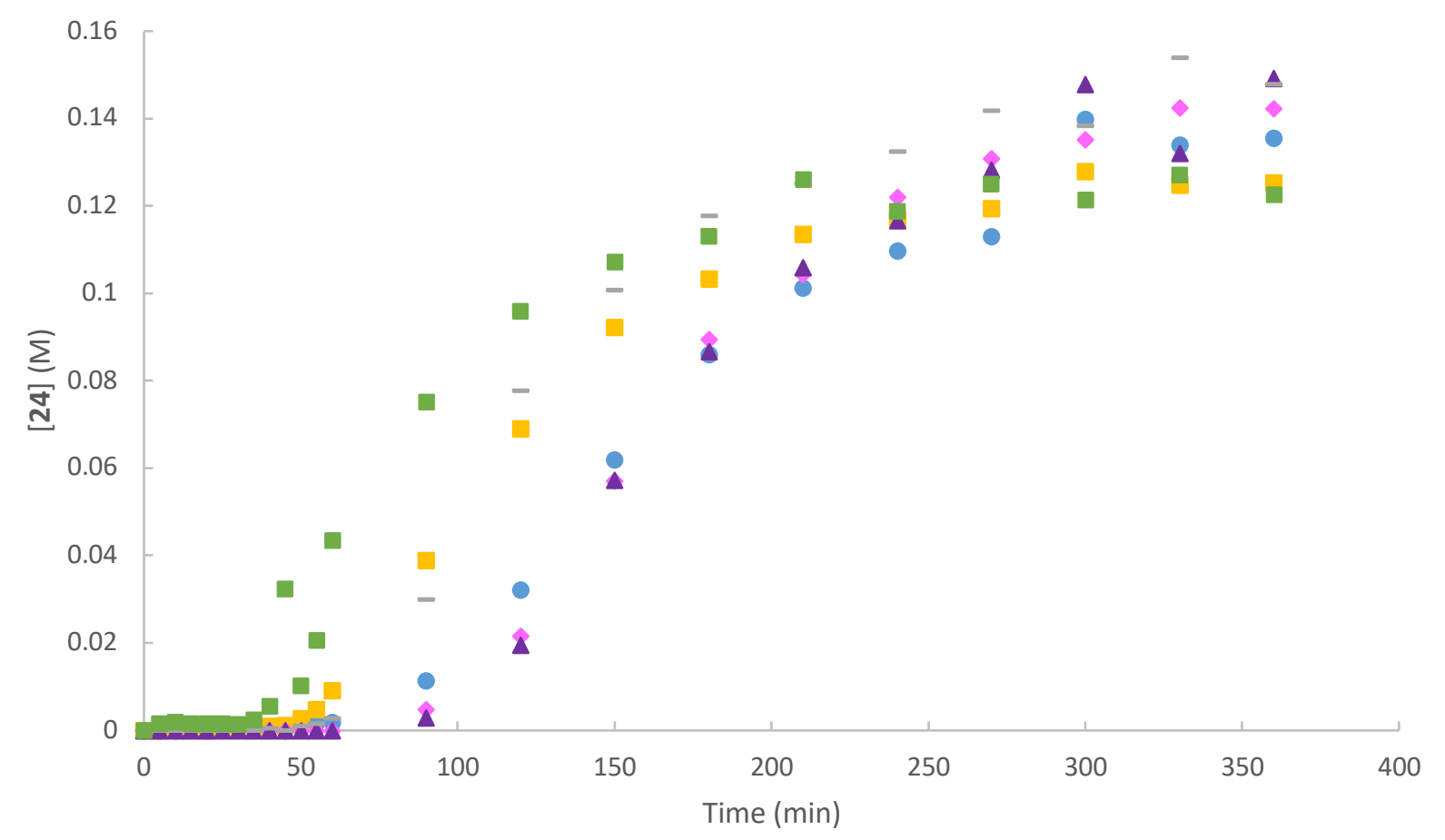

Figure S9. Comparison of reaction progress after pre-mixing ArBPin and $t$-BuOLi at the temperature and for the time noted. $\bullet$ No pre-mixing. $\square$ Pre-mixing at room temperature for 30 min. Pre-mixing at room temperature for $90 \mathrm{~min}$. - Pre-mixing at $80^{\circ} \mathrm{C}$ for $30 \mathrm{~min}$. $\Delta$ Premixing at $80{ }^{\circ} \mathrm{C}$ for $90 \mathrm{~min}$. $\square$ Pre-mixing with $s$-BuOH at room temperature for $30 \mathrm{~min}$. 
We also considered the possibility of autocatalysis and tested the addition of potential byproducts of the reaction. However, none of these eliminated the induction period. Because we observed a minor effect with $\mathrm{B}(\mathrm{O} i \text {-Pr })_{3}$, we also tested additional Lewis acids (Figure $\mathrm{S} 11$ ). For these experiments, the same procedure was followed as described at the beginning of the Reaction Progress Analysis section, with the additives added to the 2-dram vial along with the $\mathrm{Ni}(\mathrm{OAc})_{2} \cdot 4 \mathrm{H}_{2} \mathrm{O}, \mathbf{L 1}$, and $\mathrm{LiO} t-\mathrm{Bu}$.

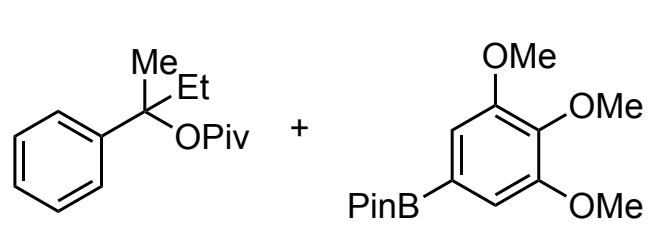

$( \pm)-1 a$

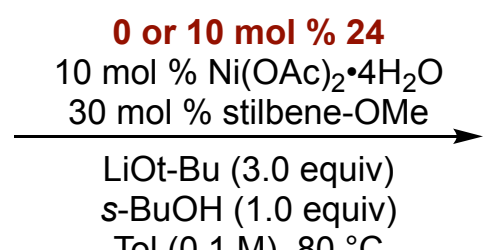

Tol (0.1 M), $80^{\circ} \mathrm{C}$

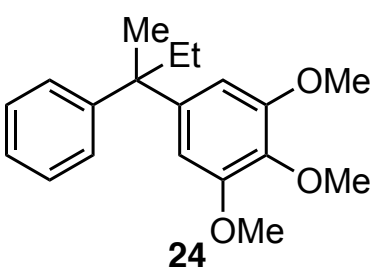

24

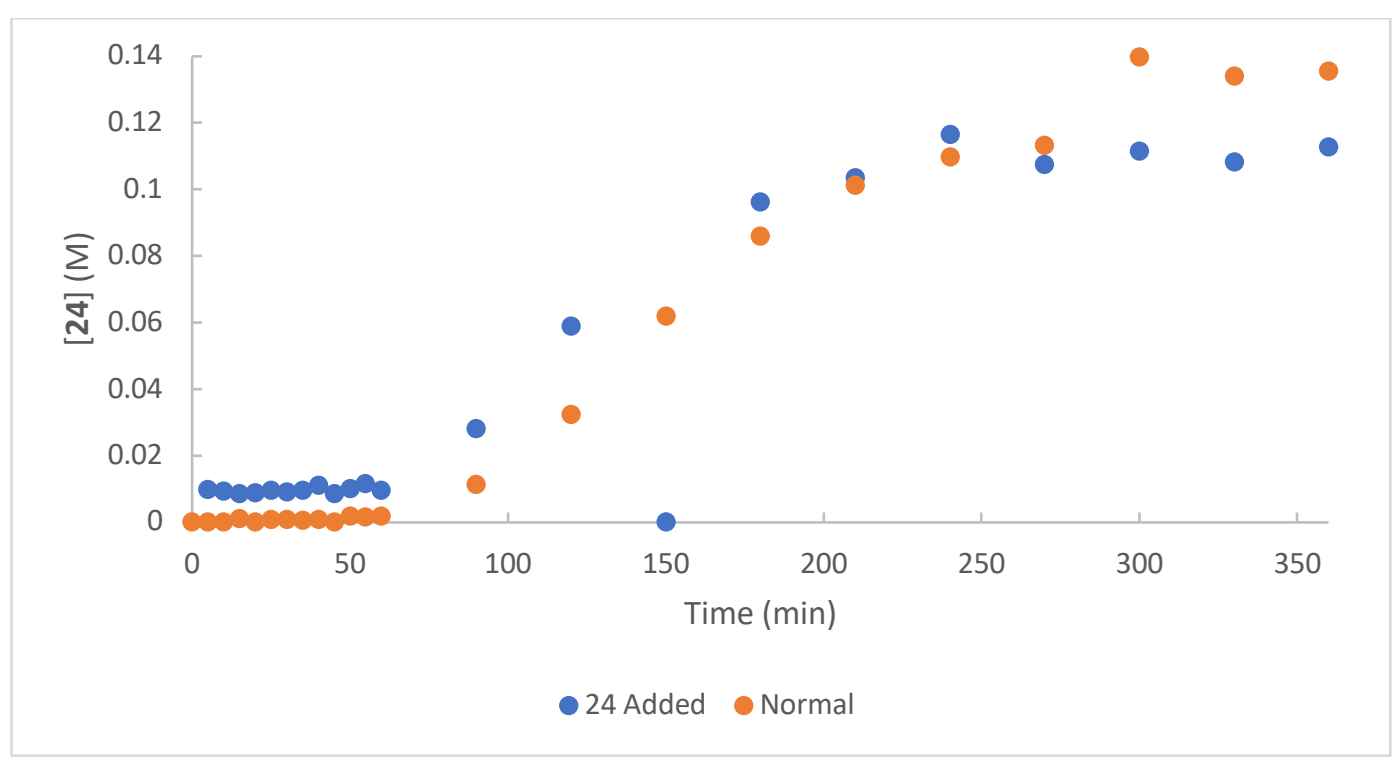

Figure S10. Comparison of reaction progress with and without $10 \mathrm{~mol} \%$ product 24 added at the beginning of the reaction. $\bullet 0$ mol \% 24 added. $\bullet 10$ mol \% 24 added. 

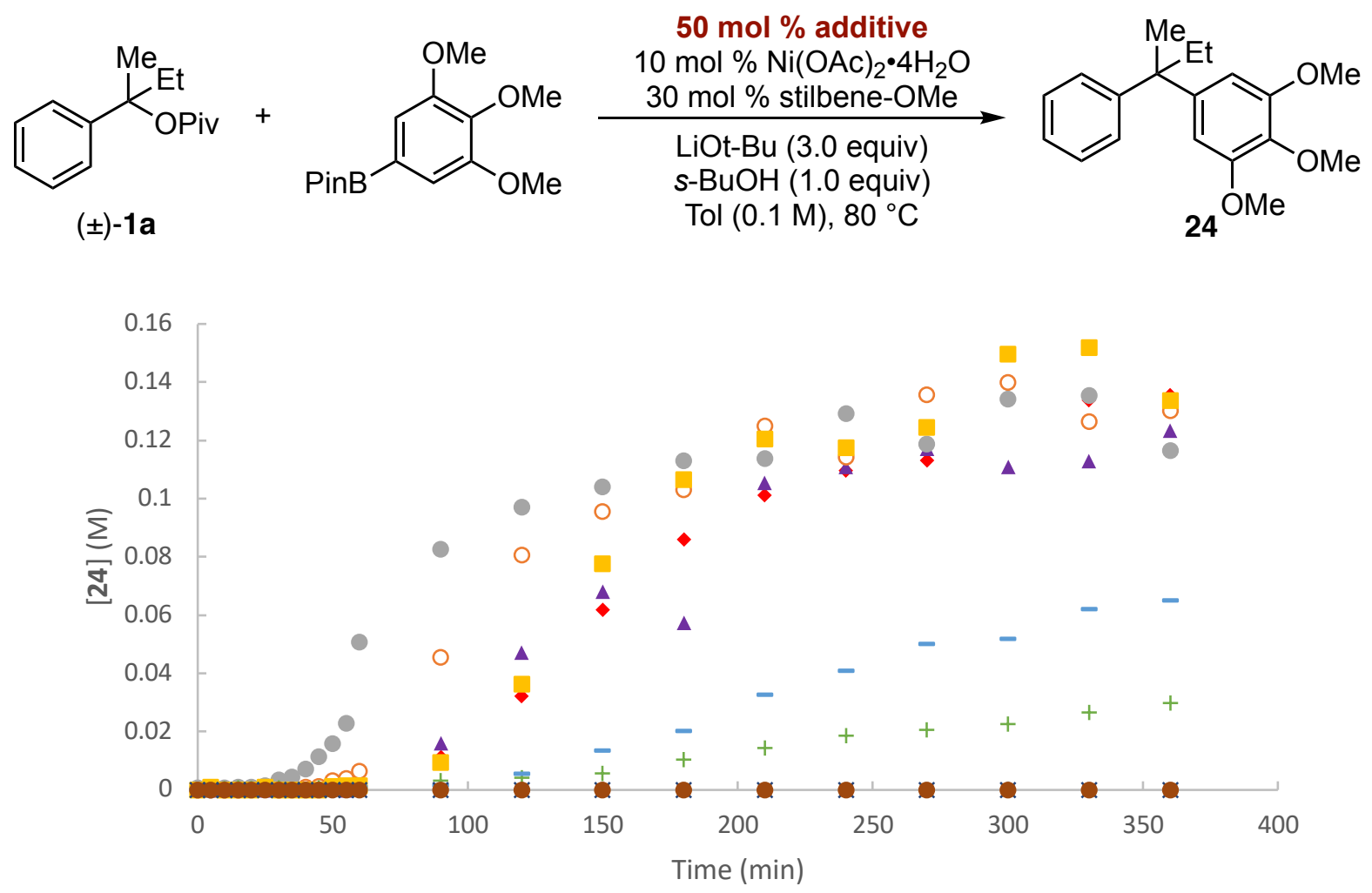

Figure S11. Comparison of reaction progress with $50 \mathrm{~mol} \%$ of various additives. $\bullet$ No additive.

$\triangle \mathrm{KOPiv} . \circ \mathrm{PinB}(\mathrm{O} i \mathrm{Pr}) . \bullet \mathrm{B}(\mathrm{O} i-\mathrm{Pr})_{3} .+\mathrm{B}\left(\mathrm{C}_{6} \mathrm{~F}_{5}\right)_{3} . \Perp \mathrm{Ph}-\mathrm{Ph} .-\mathrm{BF}_{3} \cdot \mathrm{OEt}_{2} . \times \mathrm{ZnCl}_{2} . \bullet \mathrm{AuCl}$. 


\section{Mass Recovery of L1}

Investigation of the fate of $\mathbf{L} \mathbf{1}$ under the reaction conditions showed that $>90 \%$ of $\mathbf{L} \mathbf{1}$ is recovered after the cross-coupling. As shown below, 92\% of L1 was recovered when it was used in $30 \mathrm{~mol} \%$, and $94 \%$ of $\mathbf{L 1}$ was recovered when it was used in $100 \mathrm{~mol} \%$.

It is also notable that we did not observe formation of 3,3',4,4',5,5'-hexamethoxybiphenyl, which would result from homocoupling of the aryl boronate ester.<smiles>CCC(C)(O[Na])c1ccccc1</smiles>

$( \pm)-1 a$

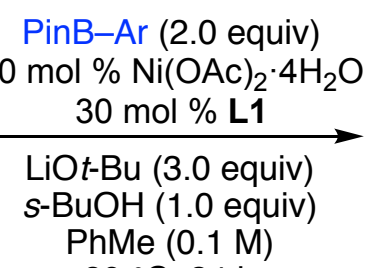
$80^{\circ} \mathrm{C}, 24 \mathrm{~h}$<smiles>CCC(C)(c1ccccc1)c1cc(OC)c(OC)c(OC)c1</smiles>

24, $85 \%$

$12 \%$ recovered $1 a$

$$
\text { PinB-Ar (2.0 equiv) }
$$<smiles>CC[C@](C)(O[Na])c1ccccc1</smiles>

1a, $96 \%$ ee

$$
\begin{gathered}
10 \mathrm{~mol} \% \mathrm{Ni}(\mathrm{OAc})_{2} \cdot 4 \mathrm{H}_{2} \mathrm{O} \\
\underset{\mathrm{LiOt}-\mathrm{Bu}(3.00 \text { equiv) }}{\mathrm{s} \text {-BuOH (1.0 equiv) }}
\end{gathered}
$$

$\mathrm{CyH}(0.2 \mathrm{M})$

$80^{\circ} \mathrm{C}, 24 \mathrm{~h}$

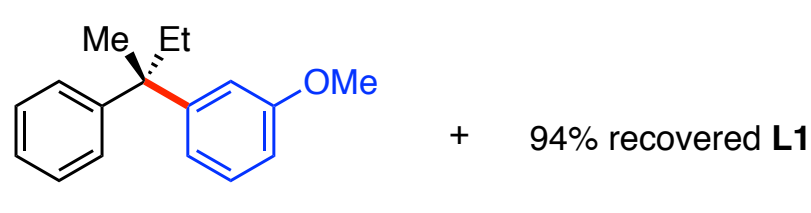

3, $93 \%$ $>99 \%$ es

Yields determined by ${ }^{1} \mathrm{H}$ NMR using 1,3,5-trimethoxybenzene as internal standard.

${ }^{a} \mathrm{Ar}-\mathrm{Ar}$ was not detected by either ${ }^{1} \mathrm{H}$ NMR or GC. 


\section{References}

(1) Mukaiyama, T.; Shintou, T.; Fukumoto, K. J. Am. Chem. Soc. 2003, 125 (35), 1053810539.

(2) Odachowski, M.; Bonet, A.; Essafi, S.; Conti-Ramsden, P.; Harvey, J. N.; Leonori, D.; Aggarwal, V. K. J. Am. Chem. Soc. 2016, 138 (30), 9521-9532.

(3) Zhou, Q.; Cobb, K. M.; Tan, T.; Watson, M. P. J. Am. Chem. Soc. 2016, 138 (37), 1205712060.

(4) Xu, J.; Pound, S. M.; Basch, C. H.; Duke, A. D.; Watson, M. P. Stereospecific, Ligand-Free Synthesis of All-Carbon Quaternary Stereocenters from Tertiary Benzylic Carboxylates. ChemRxiv 2021, preprint. https://doi.org/10.26434/chemrxiv.14403302.v1

(5) Hatano, M.; Matsumura, T.; Ishihara, K. Org. Lett. 2005, 7 (4), 573-576.

(6) Jeon, S. J.; Li, H.; Walsh, P. J. J. Am. Chem. Soc. 2005, 127 (47), 16416-16425.

(7) García, C.; Walsh, P. J. Org. Lett. 2003, 5 (20), 3641-3644.

(8) Hui, A.; Zhang, J.; Fan, J.; Wang, Z. Tetrahedron Asymmetry 2006, 17 (14), 2101-2107.

(9) Bieszczad, B.; Gilheany, D. G. Angew. Chemie - Int. Ed. 2017, 56 (15), 4272-4276.

(10) Vidal, C.; García-Álvarez, J.; Hernán-Gõmez, A.; Kennedy, A. R.; Hevia, E. Angew. Chemie Int. Ed. 2014, 53 (23), 5969-5973.

(11) Bagutski, V.; French, R. M.; Aggarwal, V. K. Angew. Chemie - Int. Ed. 2010, 49 (30), 51425145. 


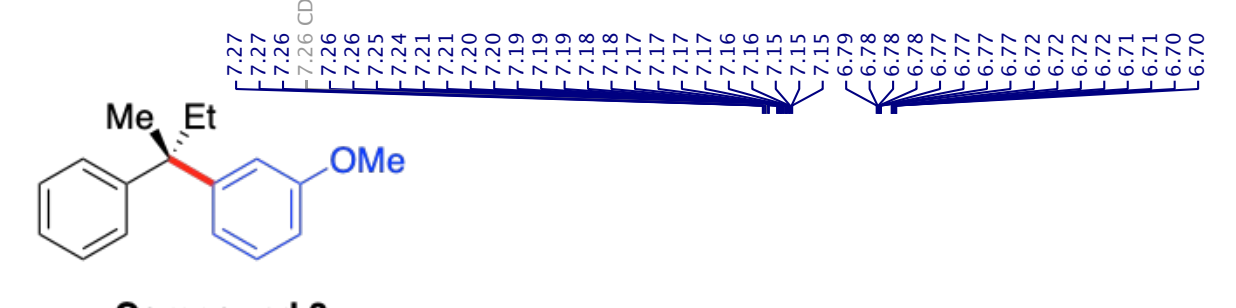

Compound 3

${ }^{1} \mathrm{H}$ NMR (600 MHz, $\mathrm{CDCl}_{3}$ )

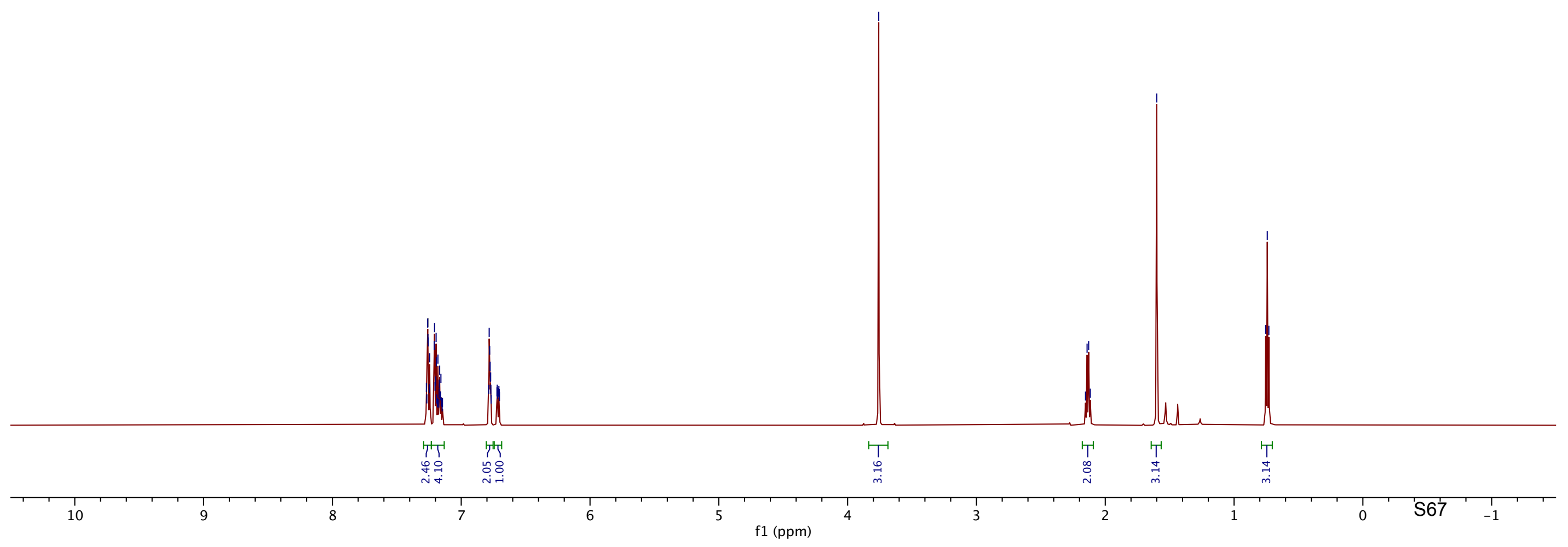




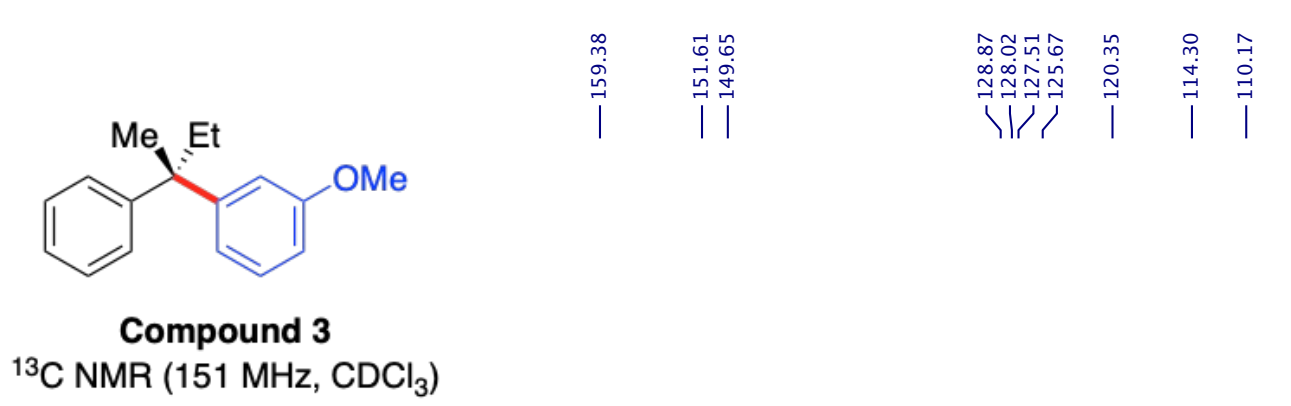

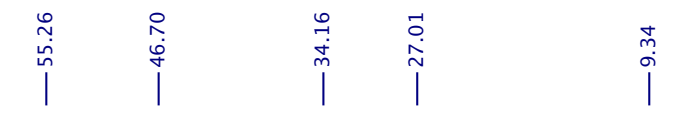
${ }^{13} \mathrm{C}$ NMR $\left(151 \mathrm{MHz}, \mathrm{CDCl}_{3}\right)$

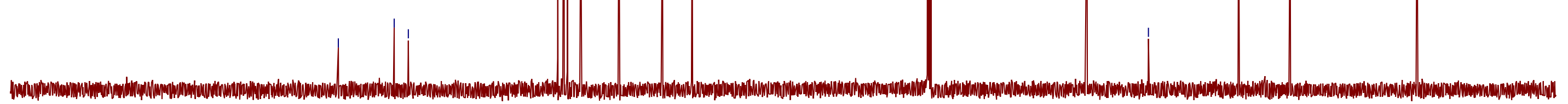

\begin{tabular}{|c|c|}
\hline 200 & 180 \\
\hline
\end{tabular}


Compound 4

${ }^{1} \mathrm{H} \mathrm{NMR}\left(600 \mathrm{MHz}, \mathrm{CDCl}_{3}\right)$

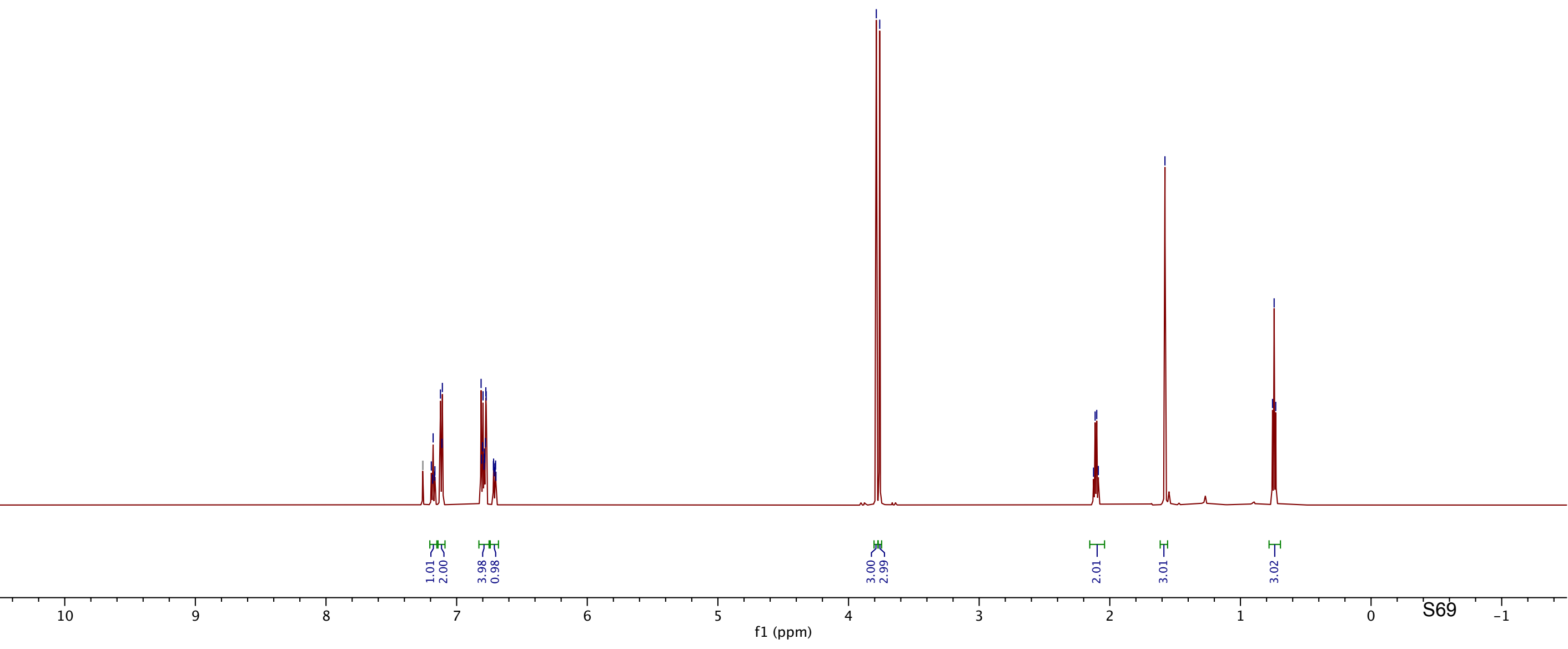




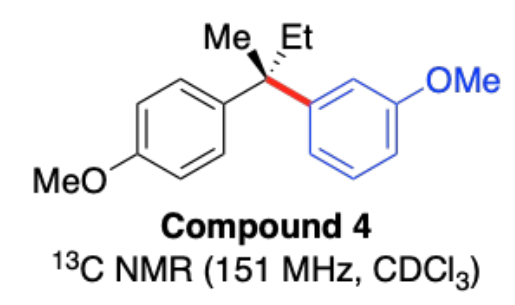

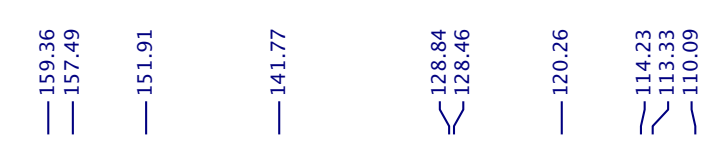

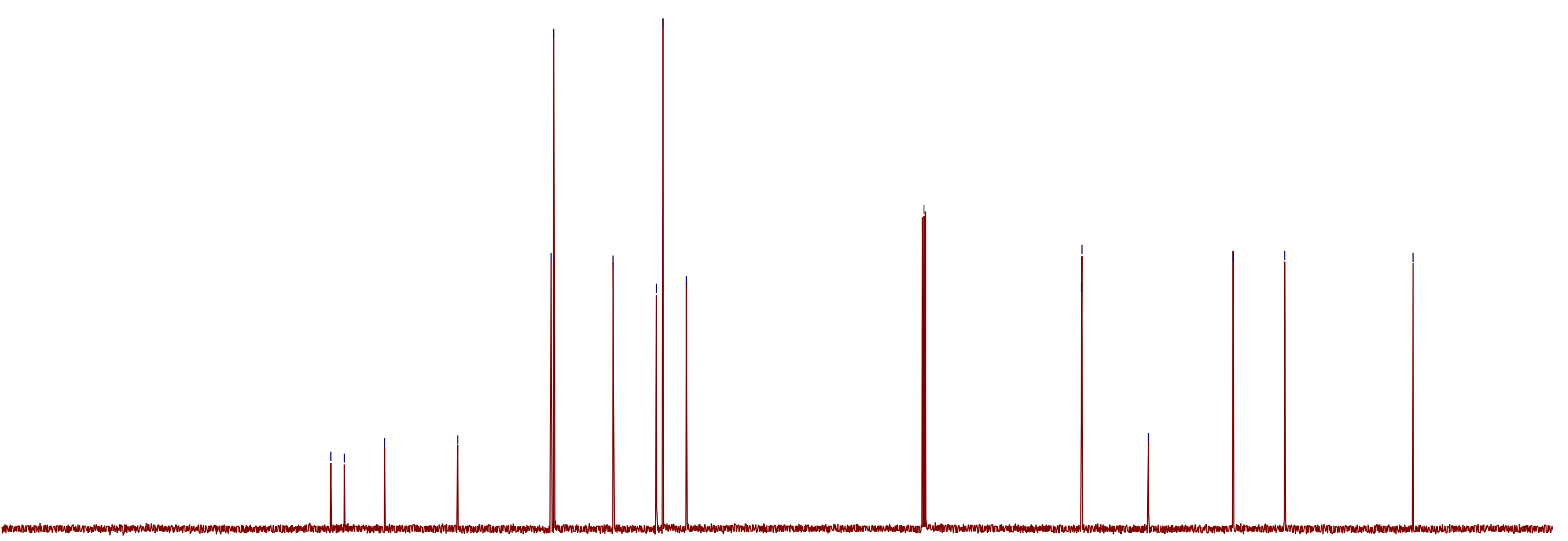




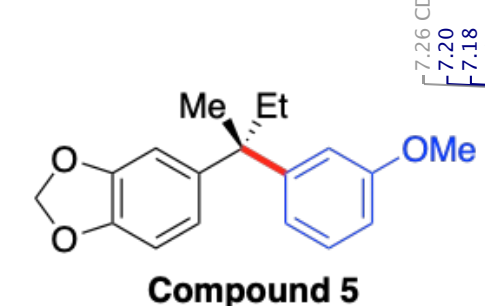

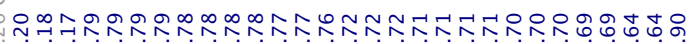

MR (600 $\mathrm{MHz}$

${ }^{1} \mathrm{H}$ NMR (600 MHz, $\mathrm{CDCl}_{3}$ )

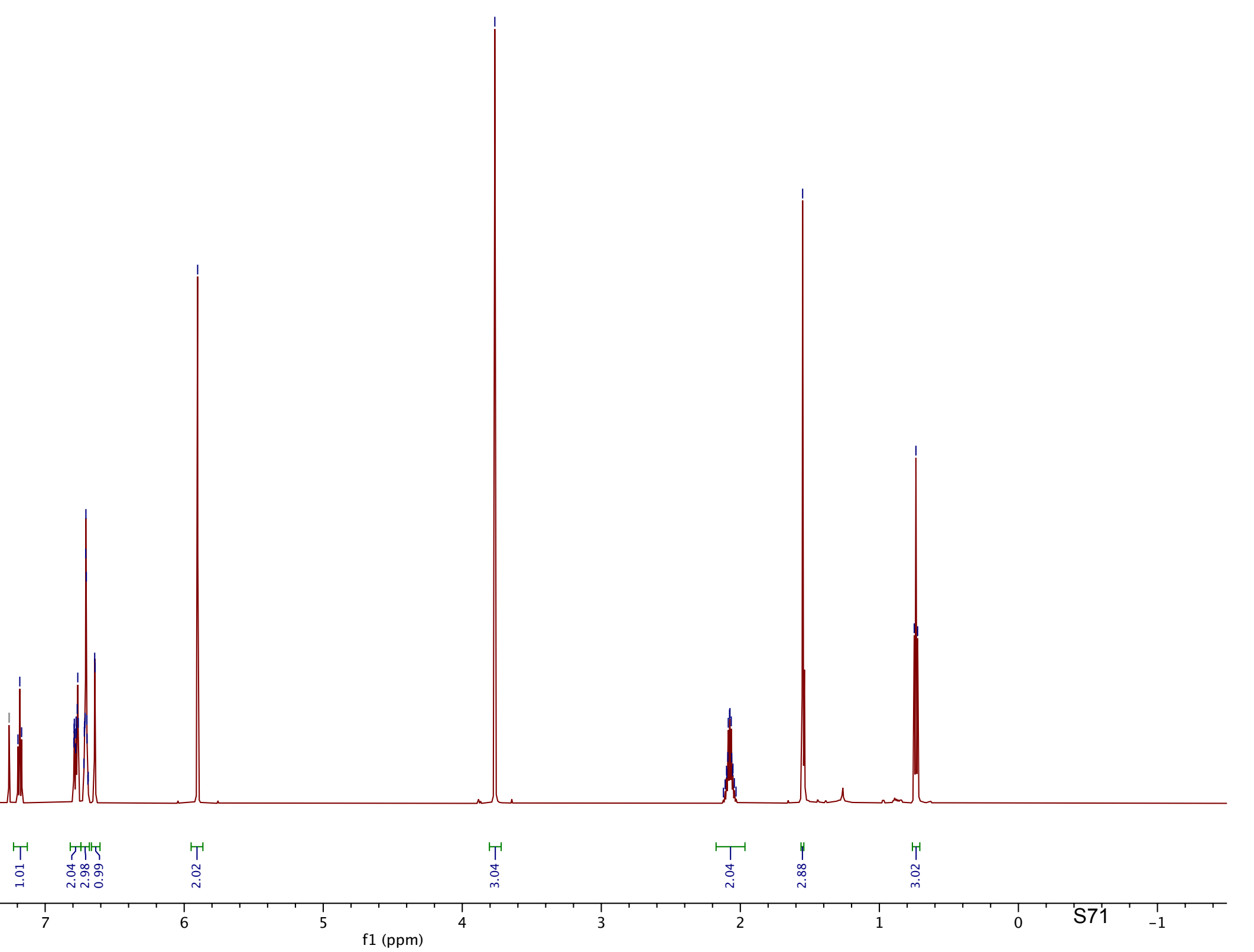




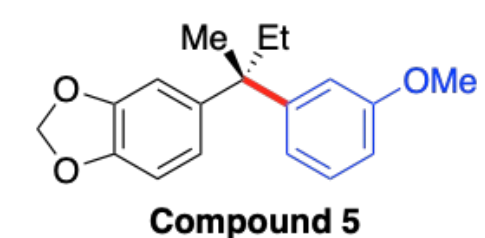

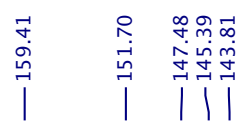

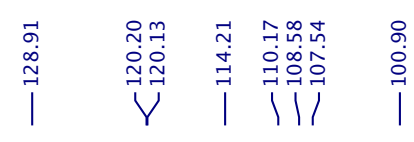

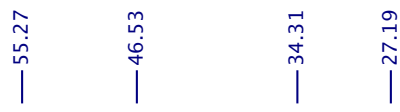

${ }^{13} \mathrm{C} \mathrm{NMR} \mathrm{(151} \mathrm{MHz,} \mathrm{CDCl}_{3}$ ) 

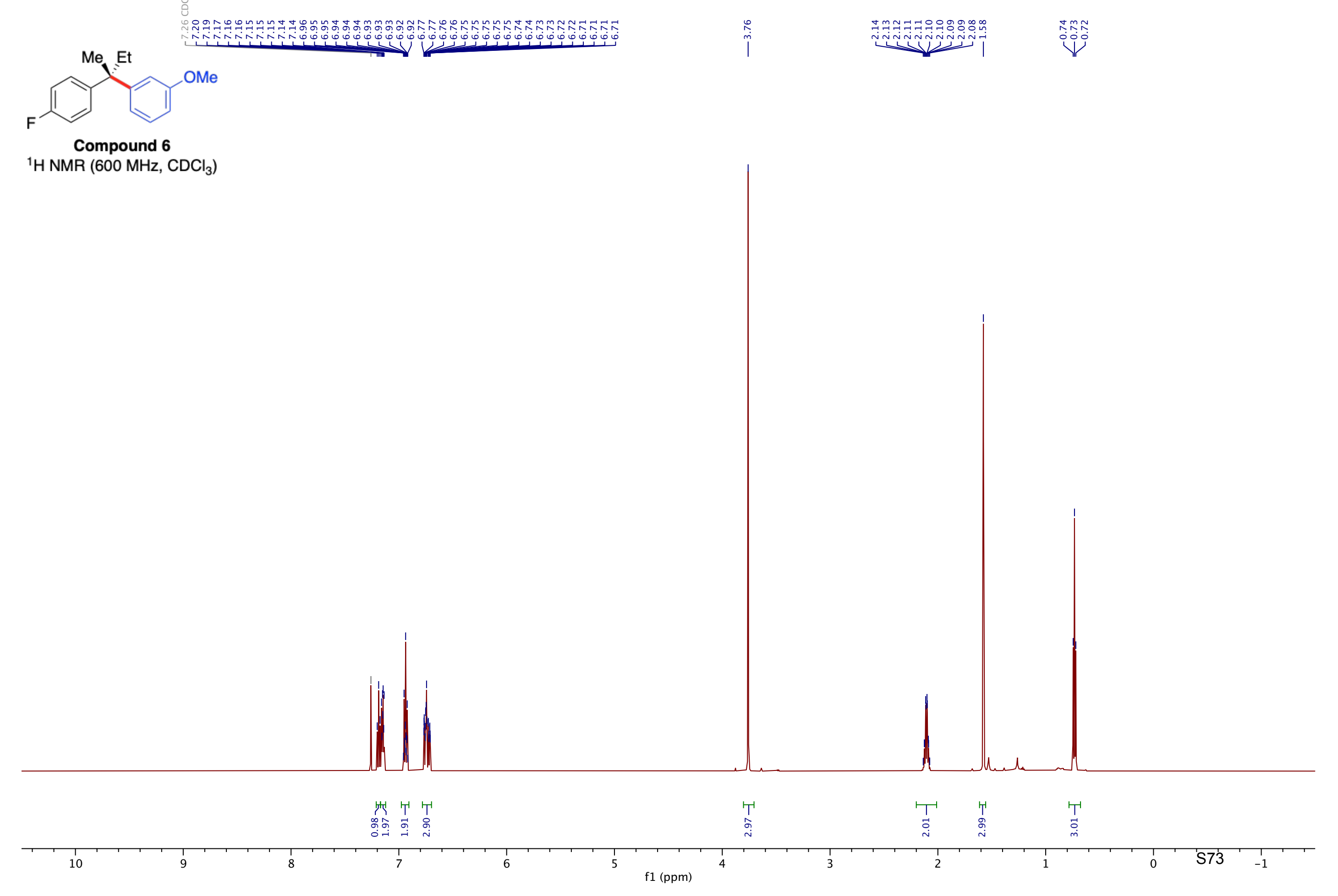


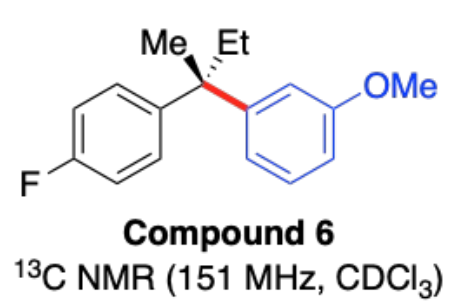

${ }^{13} \mathrm{C}$ NMR $\left(151 \mathrm{MHz}, \mathrm{CDCl}_{3}\right)$

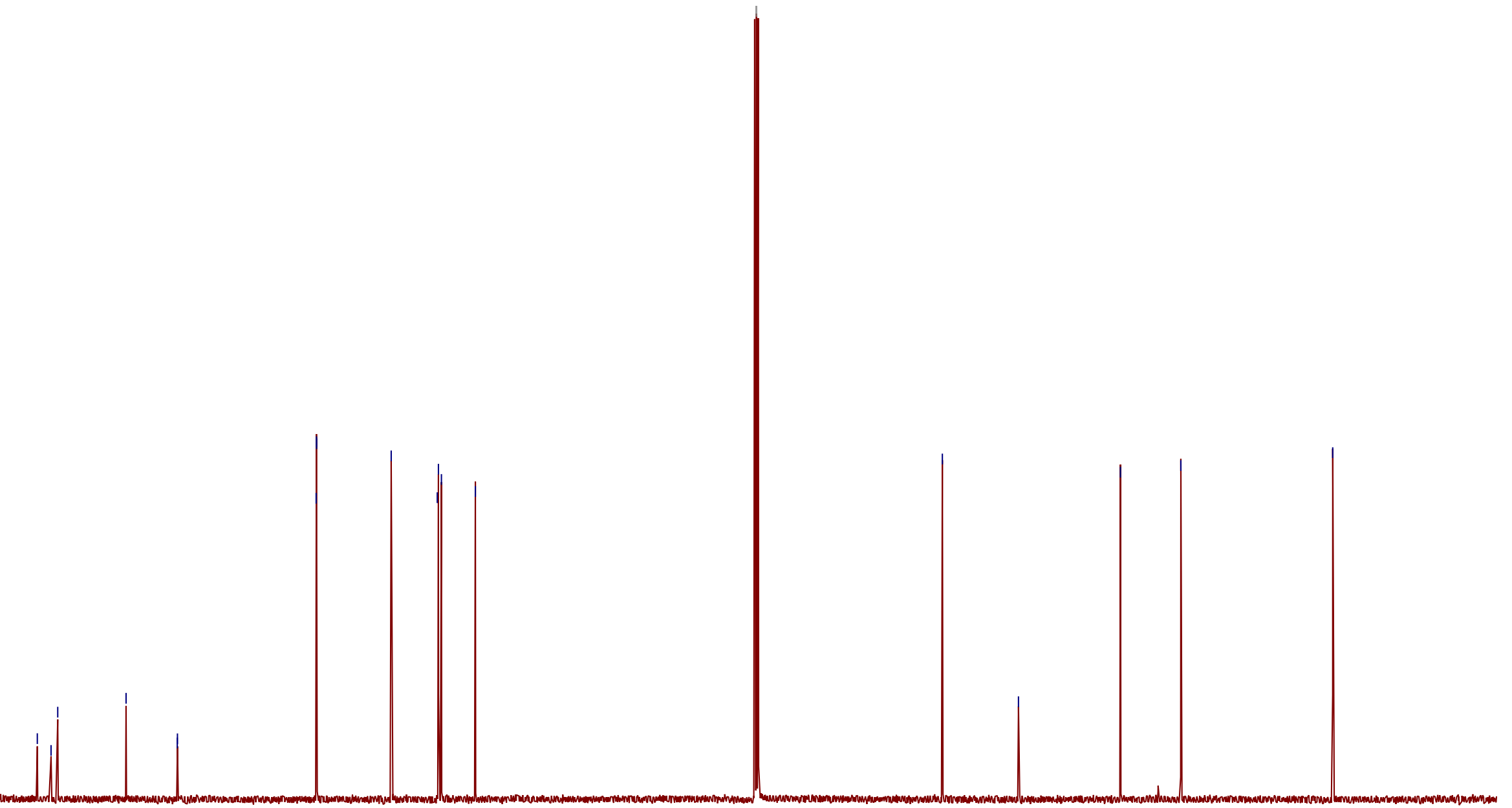

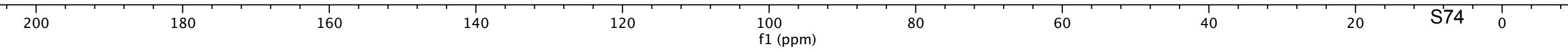




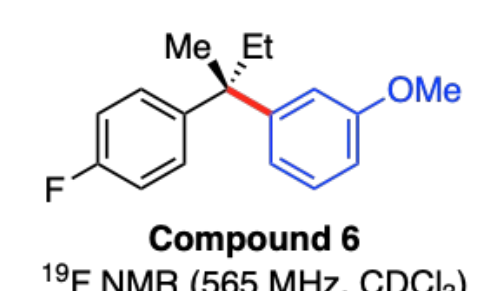

${ }^{19} \mathrm{~F}$ NMR (565 MHz, $\mathrm{CDCl}_{3}$ )

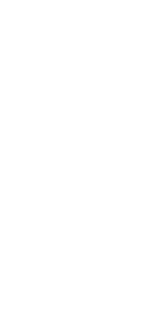



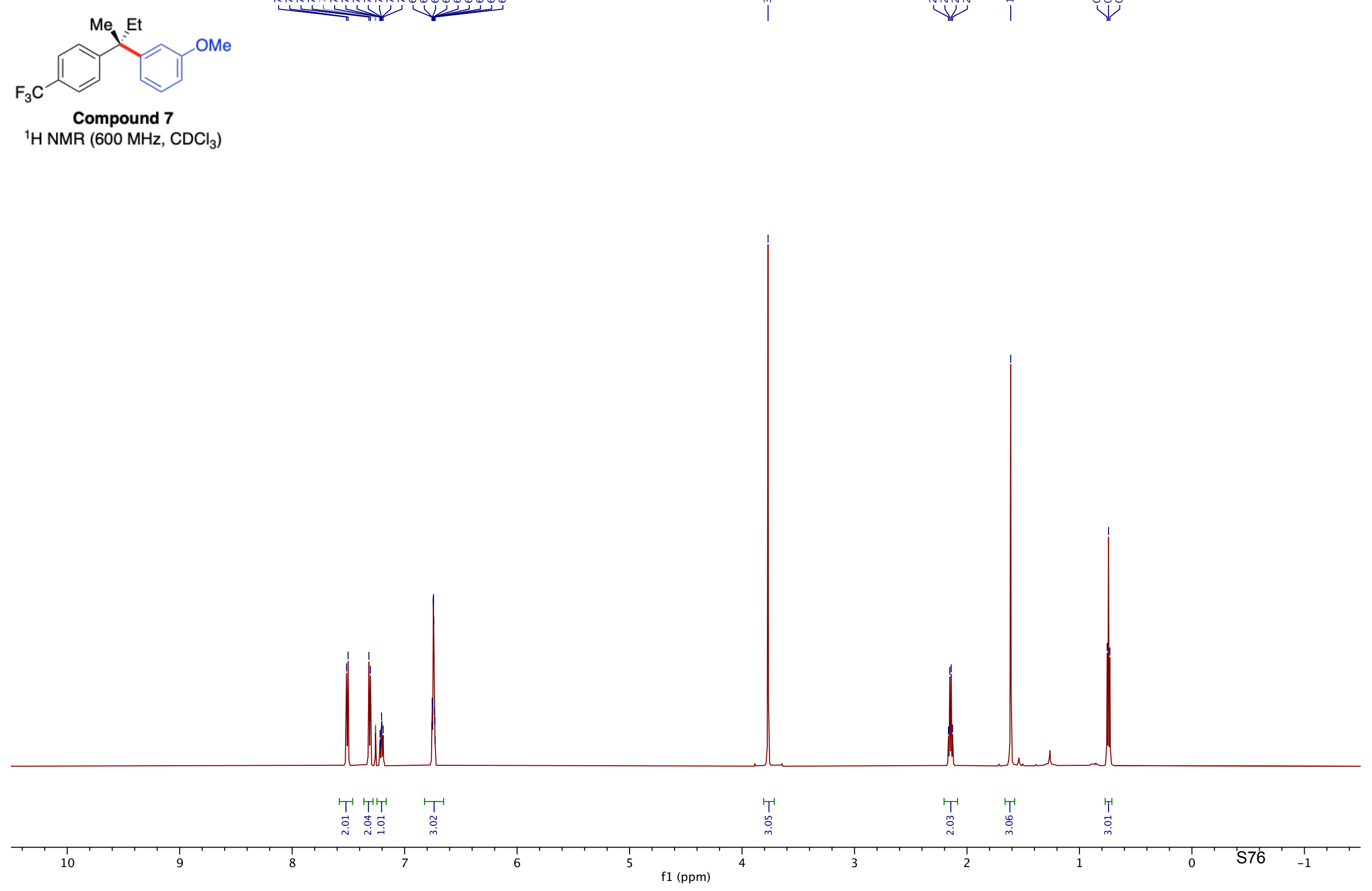


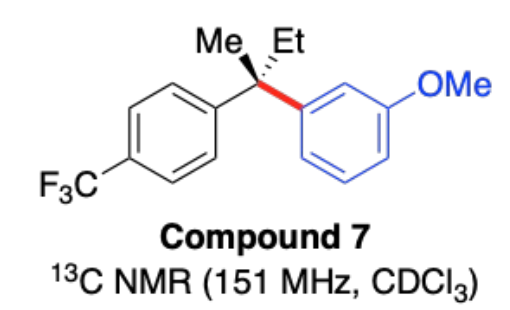

${ }^{13} \mathrm{C}$ NMR (151 MHz, $\mathrm{CDCl}_{3}$ )

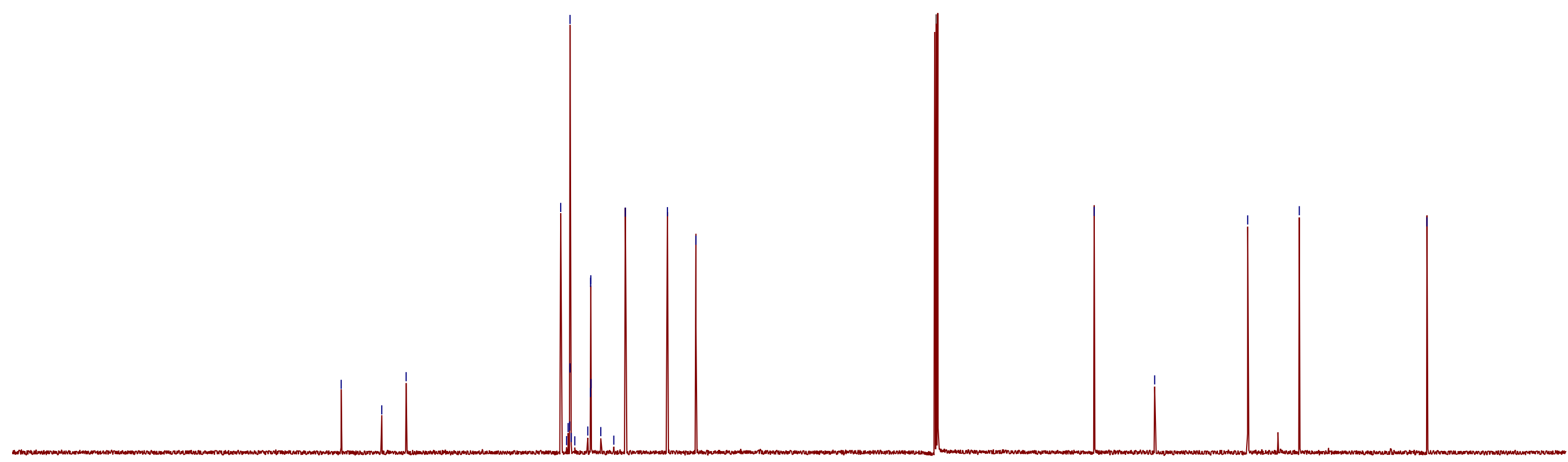




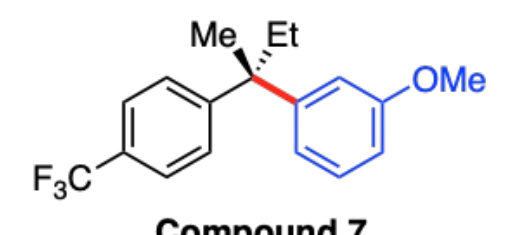

Compound 7

${ }^{19} \mathrm{~F}$ NMR (565 MHz, $\mathrm{CDCl}_{3}$ ) 


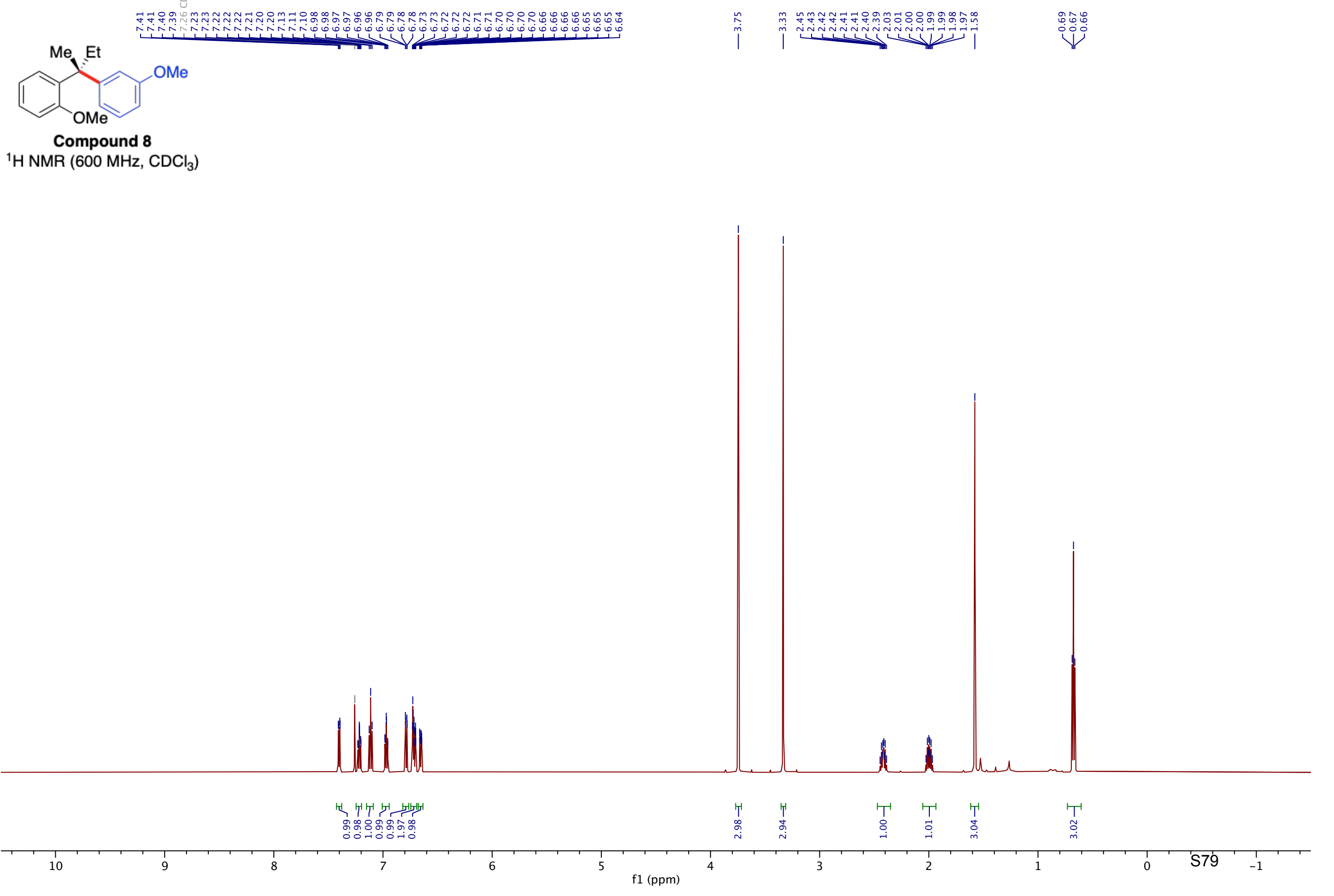




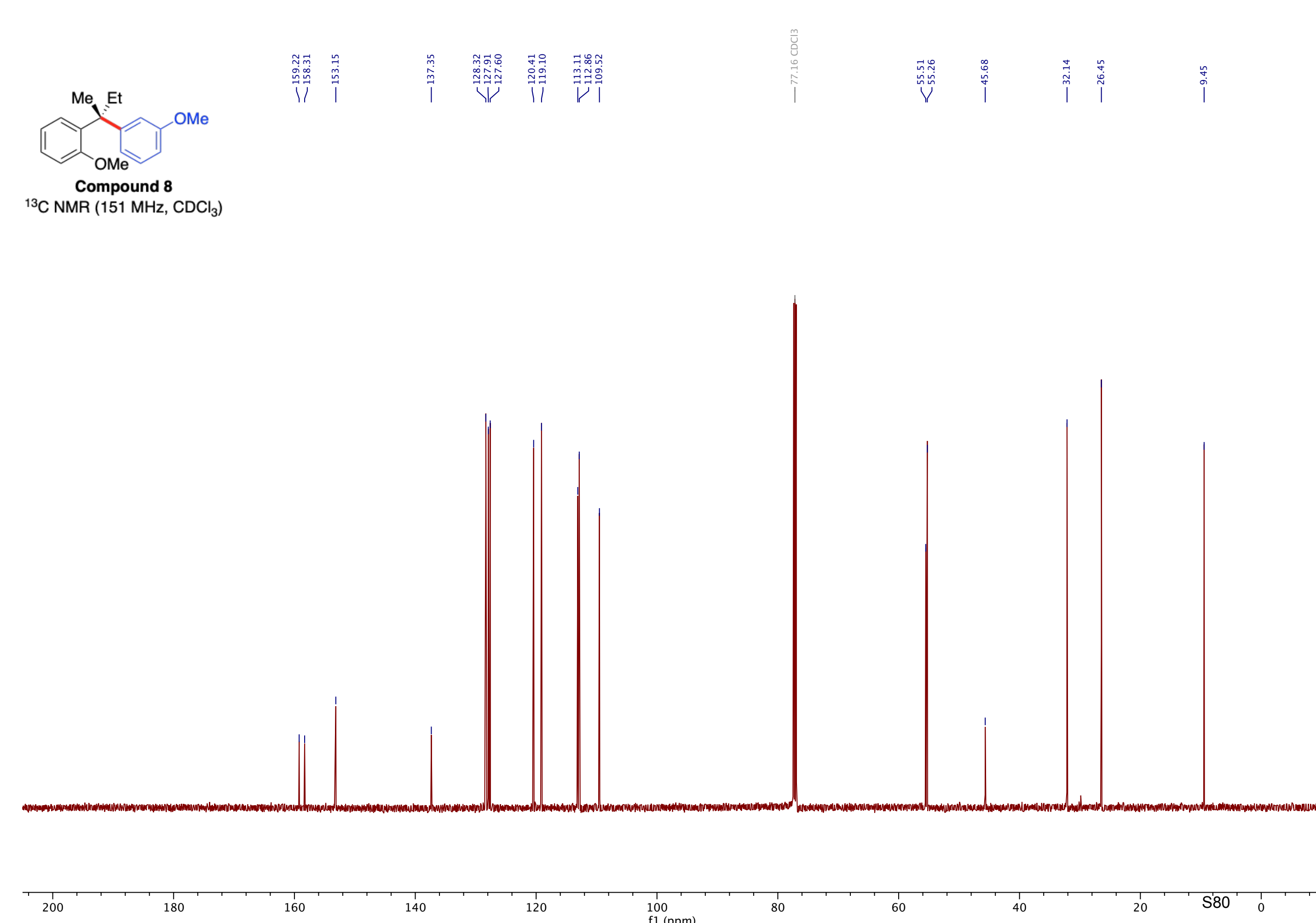




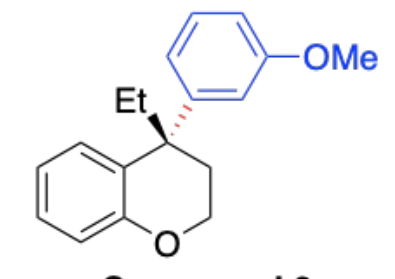

Compound 9

${ }^{1} \mathrm{H} \mathrm{NMR}\left(600 \mathrm{MHz}, \mathrm{CDCl}_{3}\right)$

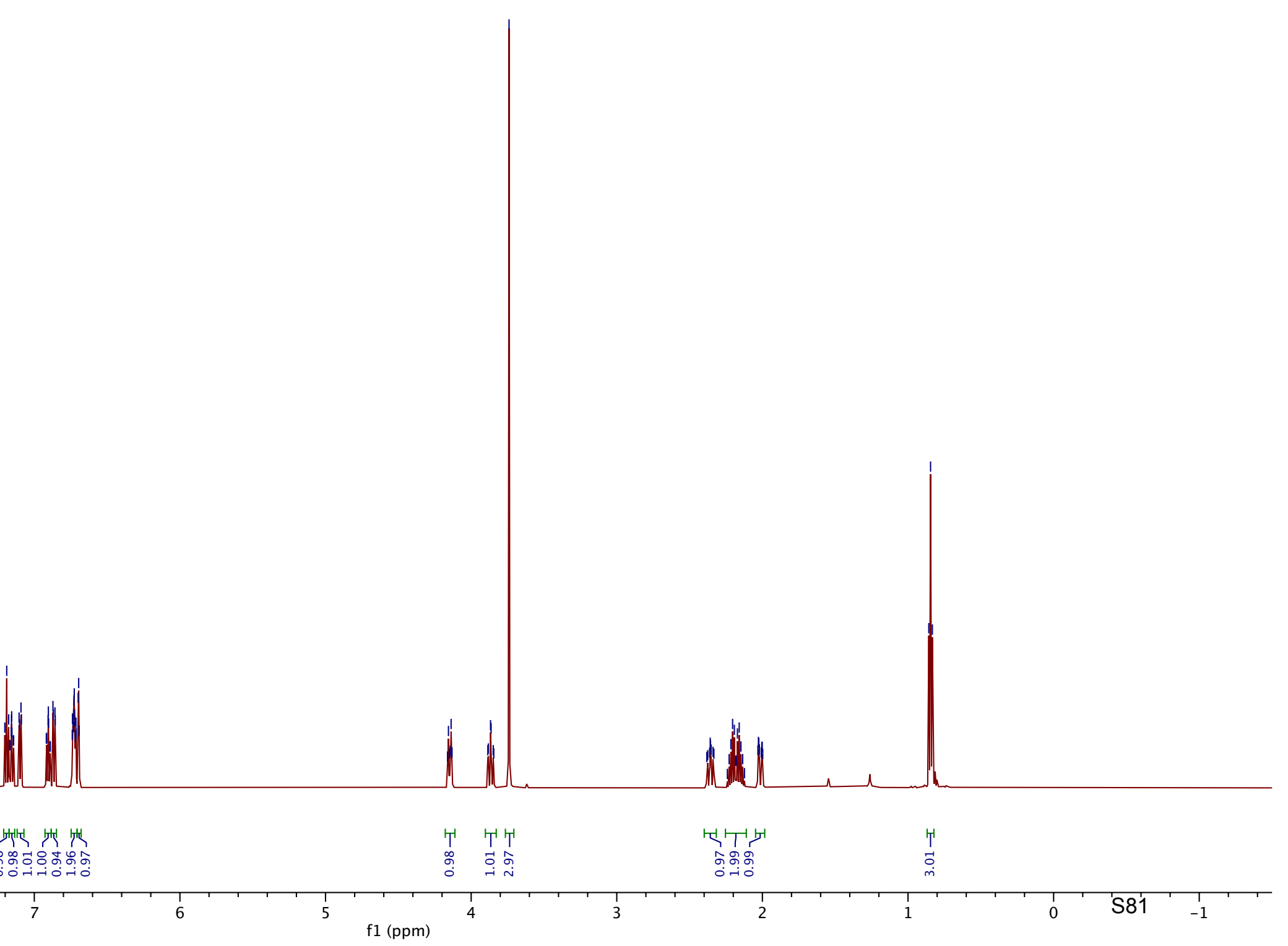




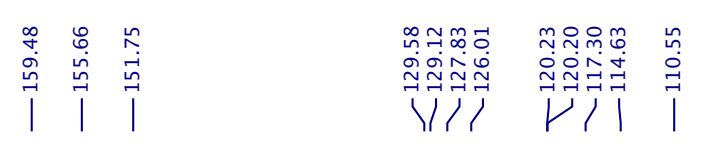

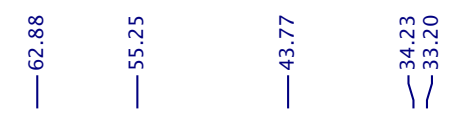

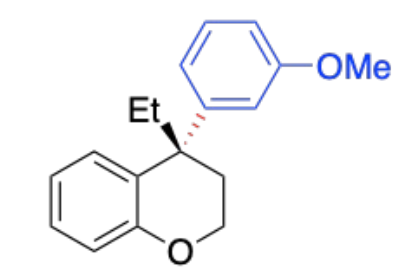

Compound 9

${ }^{13} \mathrm{C}$ NMR $\left(151 \mathrm{MHz}, \mathrm{CDCl}_{3}\right)$
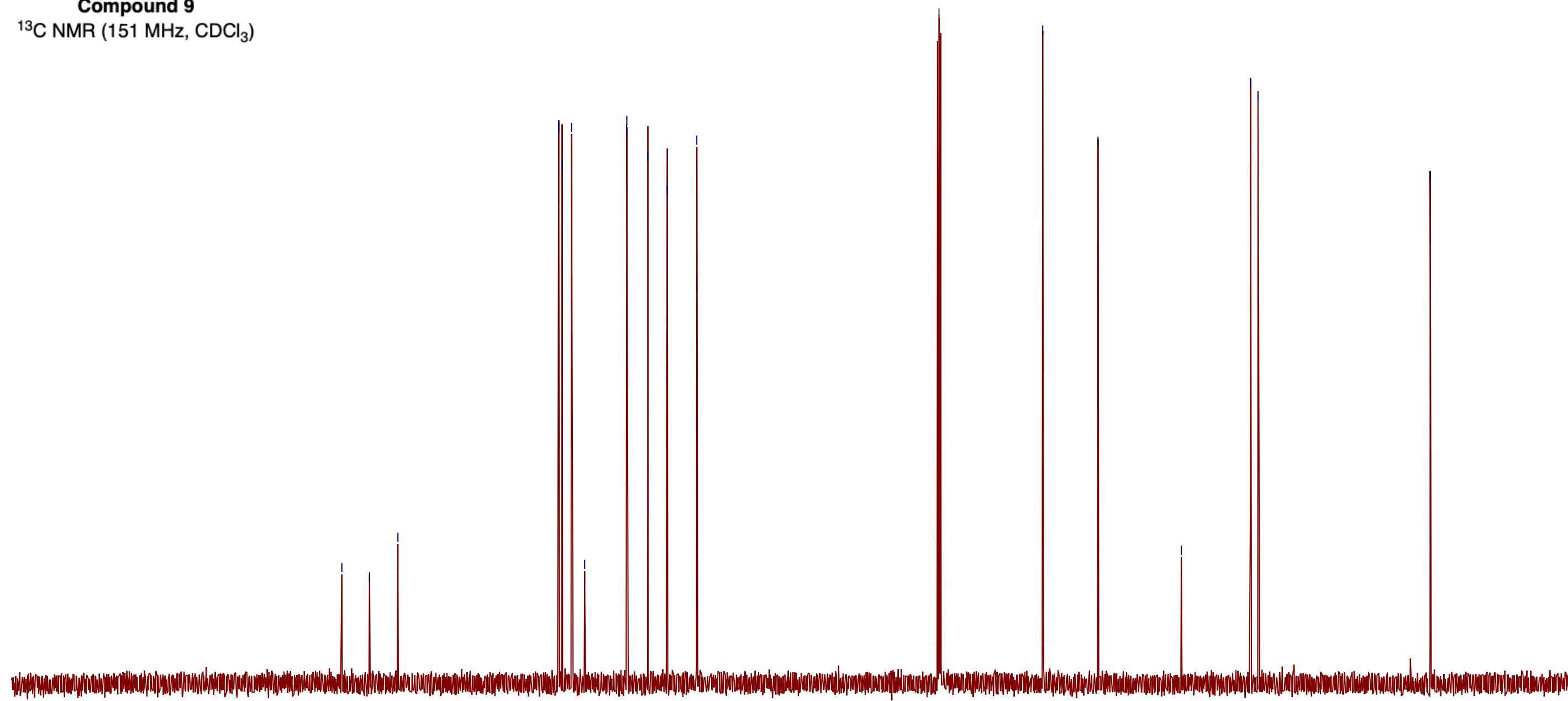

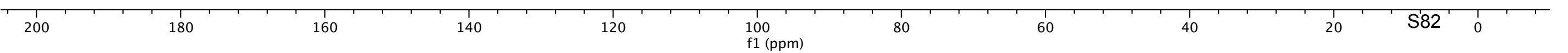




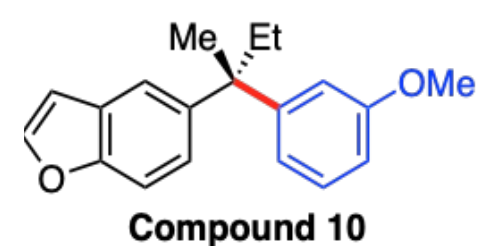

${ }^{1} \mathrm{H} \mathrm{NMR}\left(600 \mathrm{MHz}, \mathrm{CDCl}_{3}\right)$

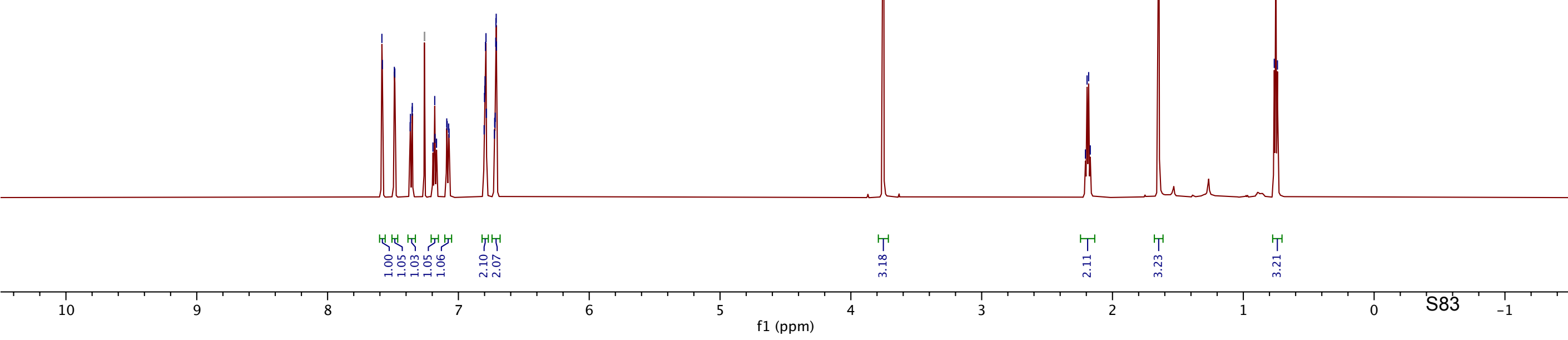




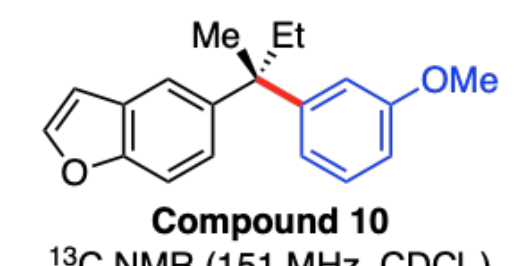

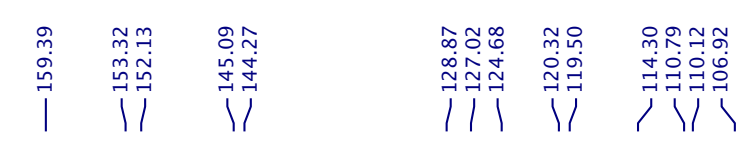

\section{${ }^{13} \mathrm{C}$ NMR $\left(151 \mathrm{MHz}, \mathrm{CDCl}_{3}\right)$}

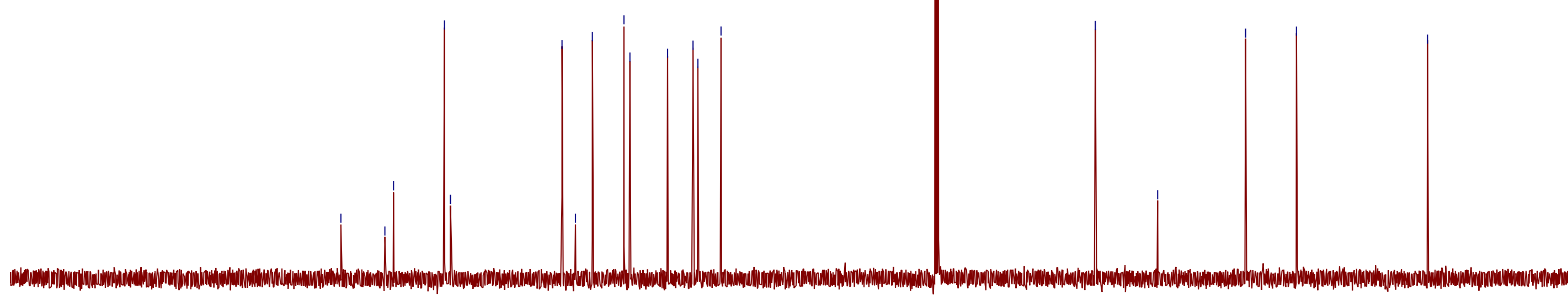

180
200




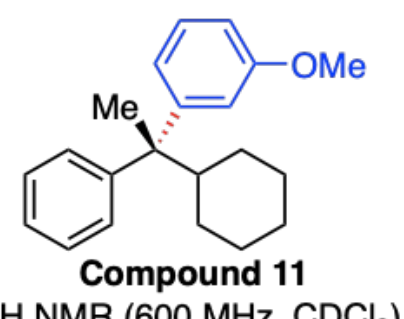

${ }^{1} \mathrm{H}$ NMR $\left(600 \mathrm{MHz}, \mathrm{CDCl}_{3}\right)$

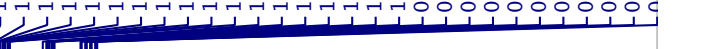

(

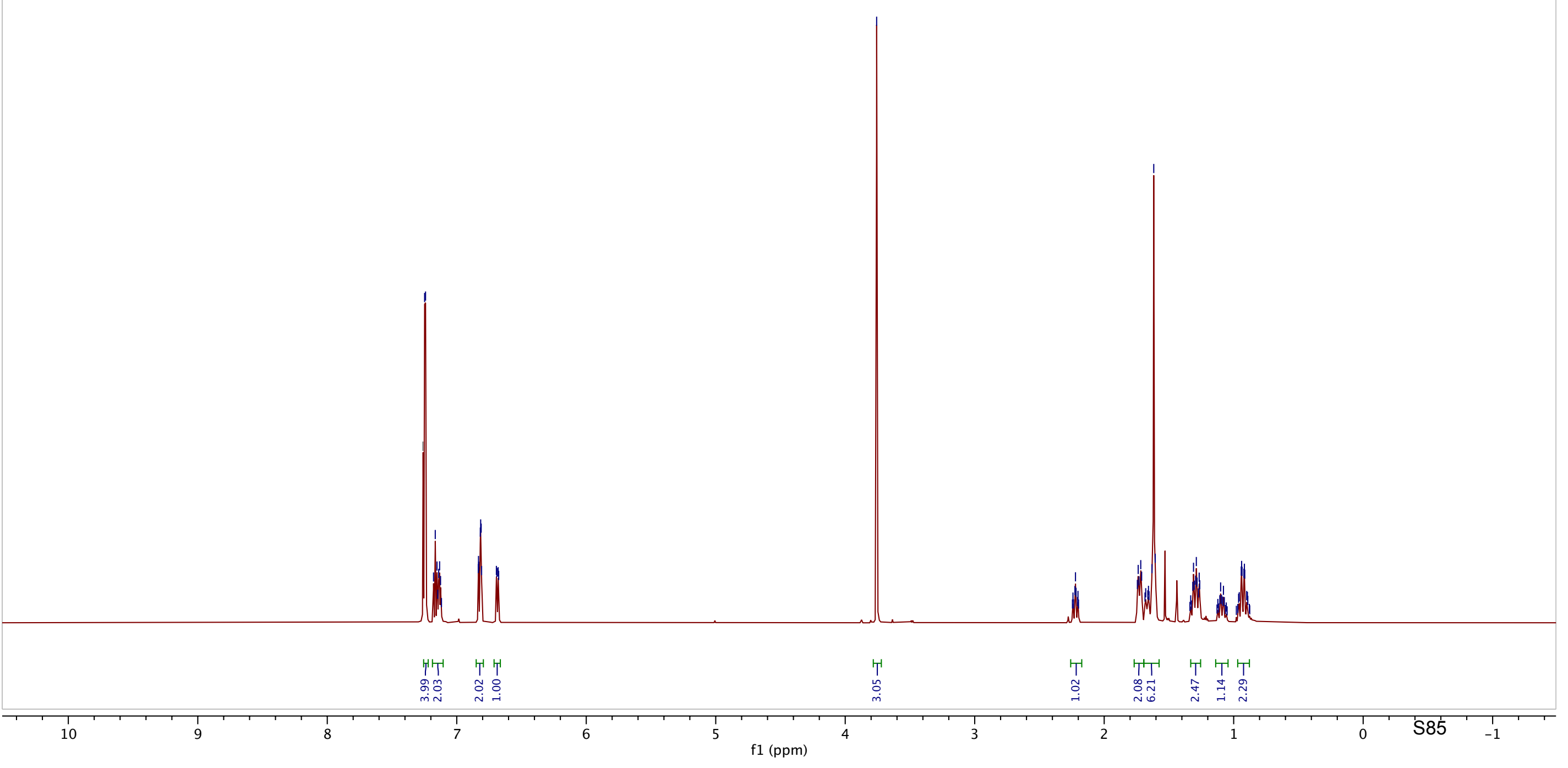


<smiles>COc1cccc([C@@](C)(c2ccccc2)C2CCCCC2)c1</smiles>

Compound 11

${ }^{13} \mathrm{C}$ NMR $\left(151 \mathrm{MHz}, \mathrm{CDCl}_{3}\right)$
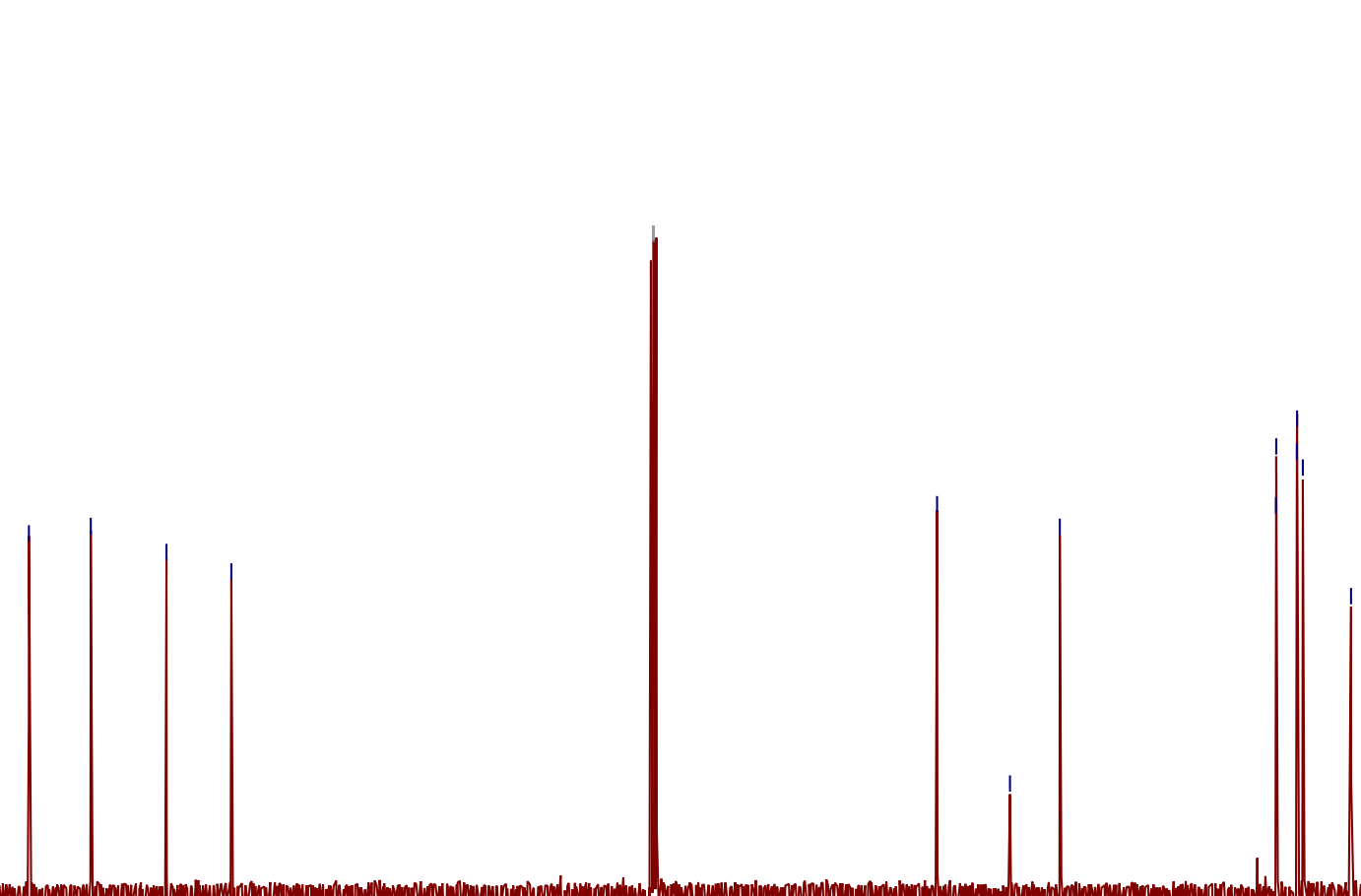

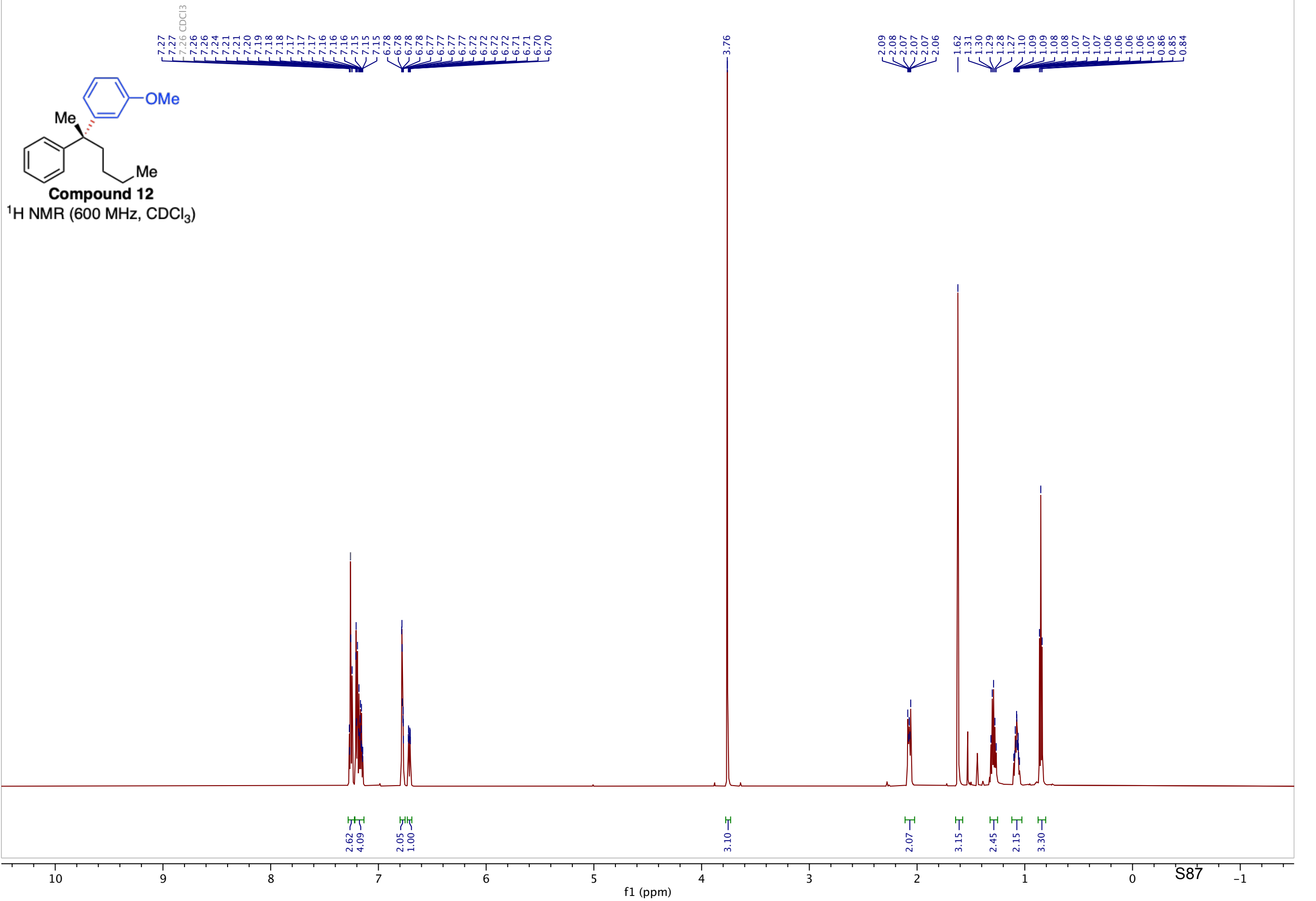


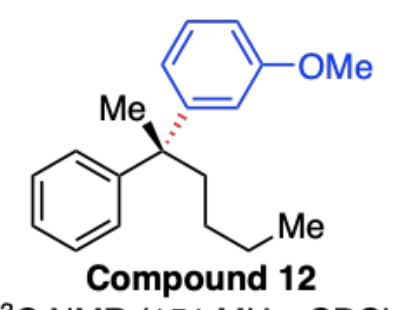

${ }^{13} \mathrm{C}$ NMR (151 MHz, $\mathrm{CDCl}_{3}$ )

\section{(151}

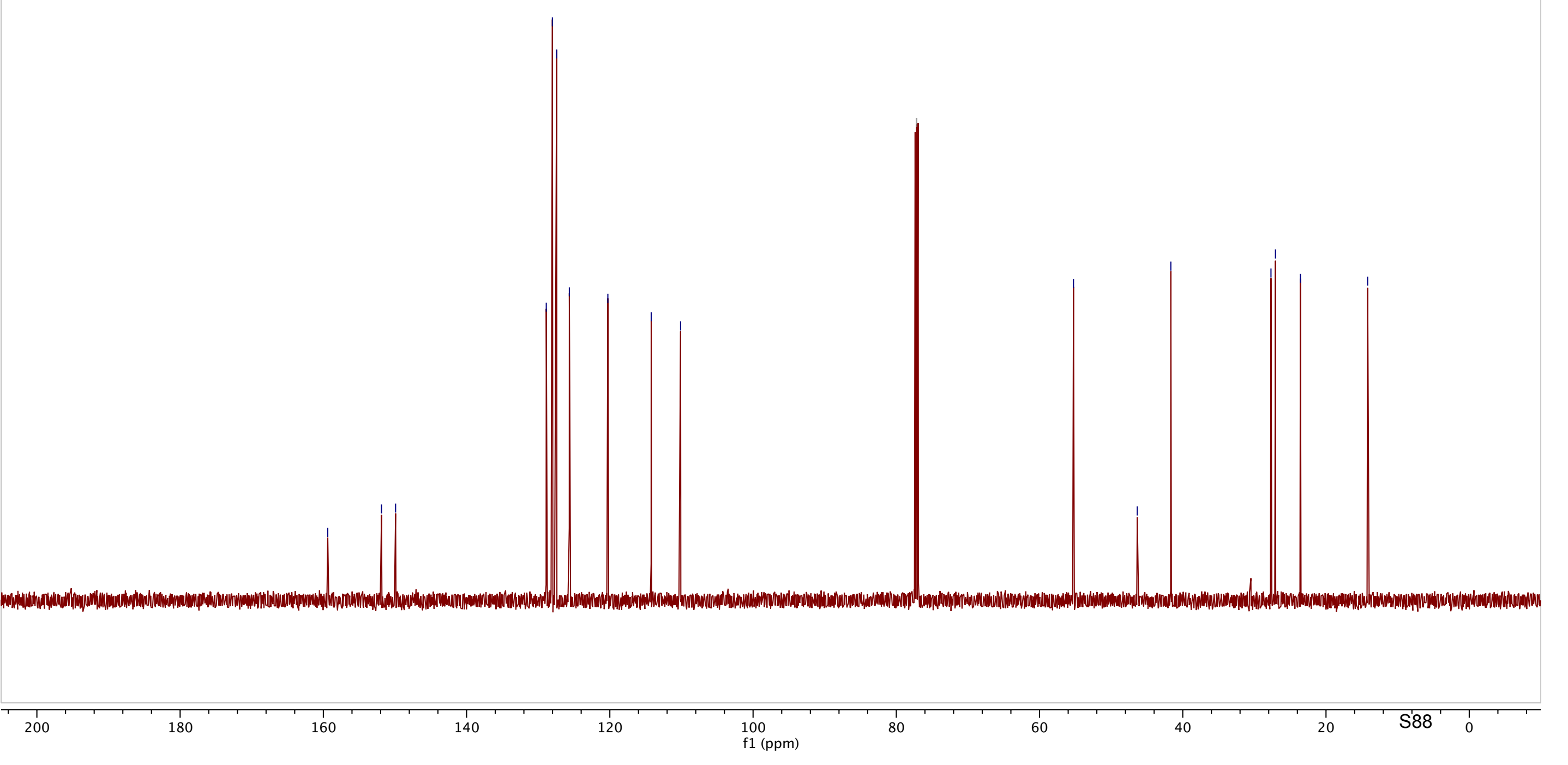



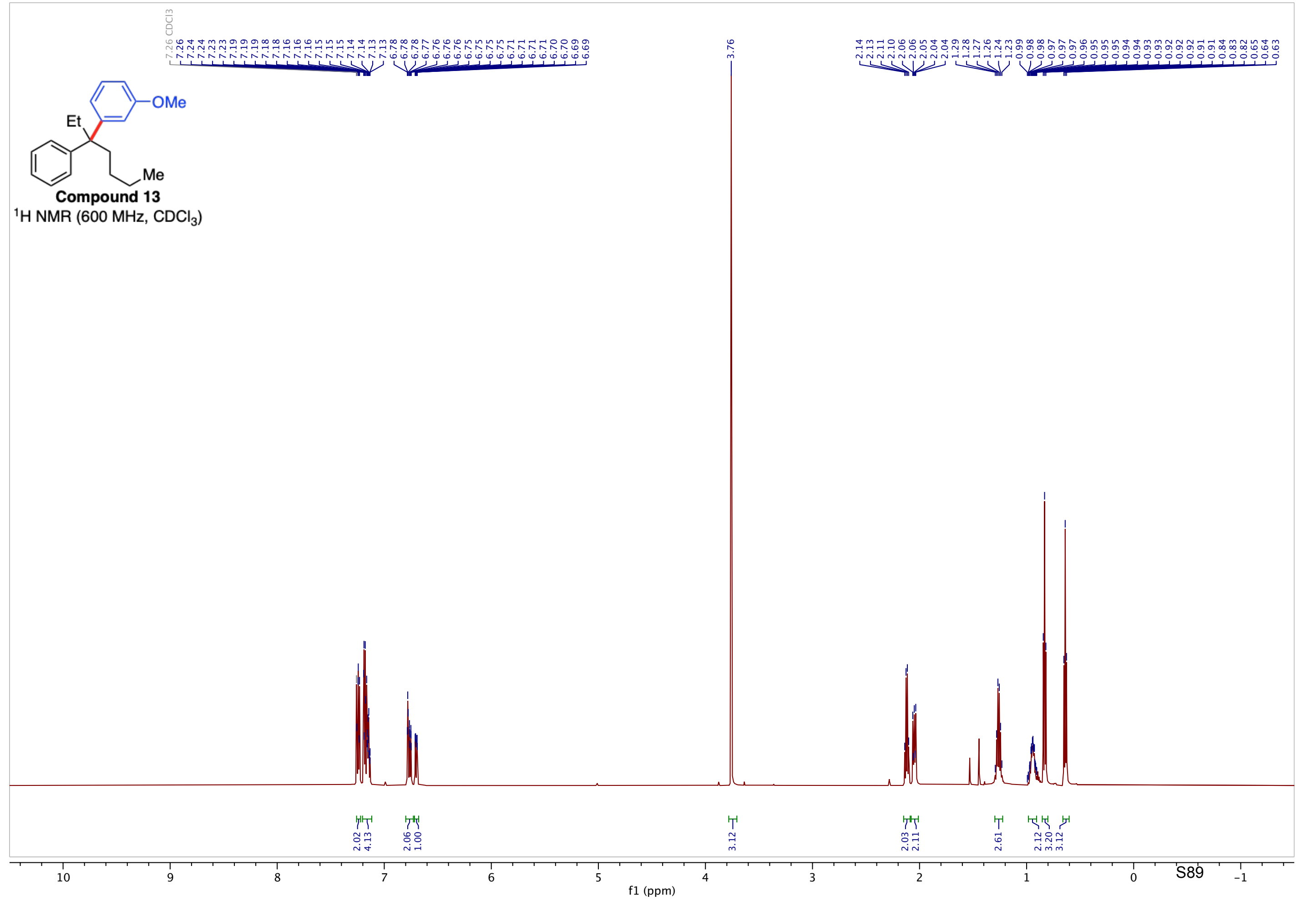


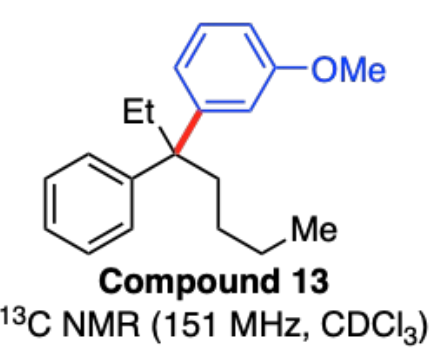

${ }^{13} \mathrm{C}$ NMR (151 MHz, $\mathrm{CDCl}_{3}$ )

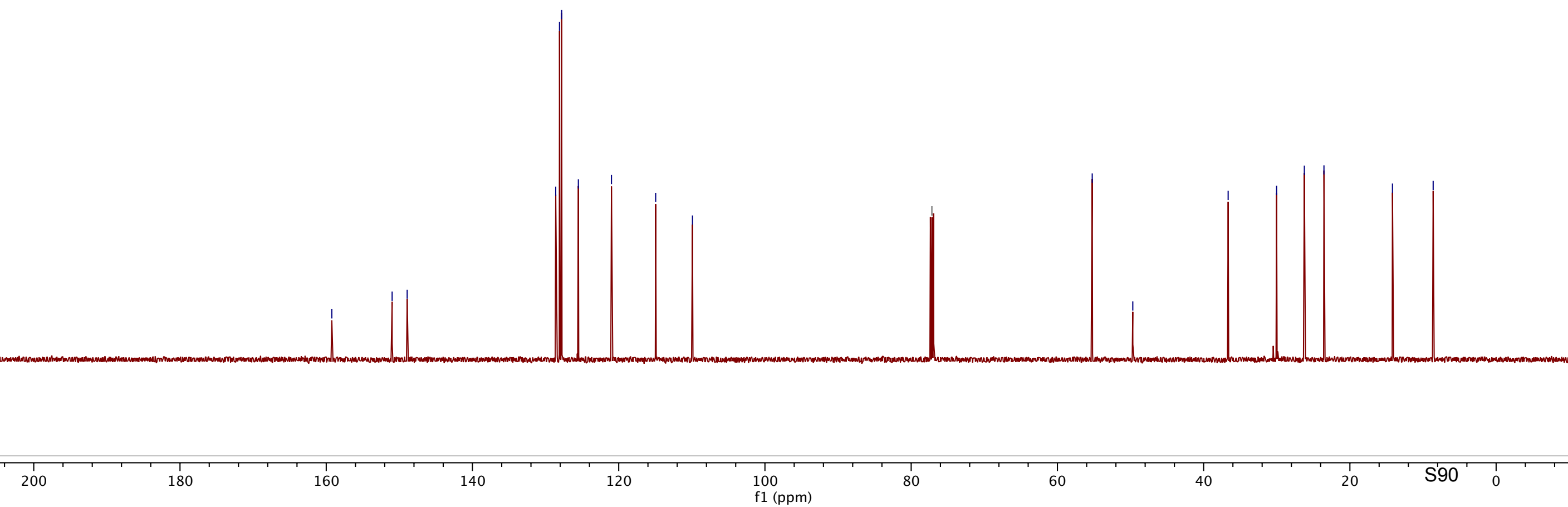



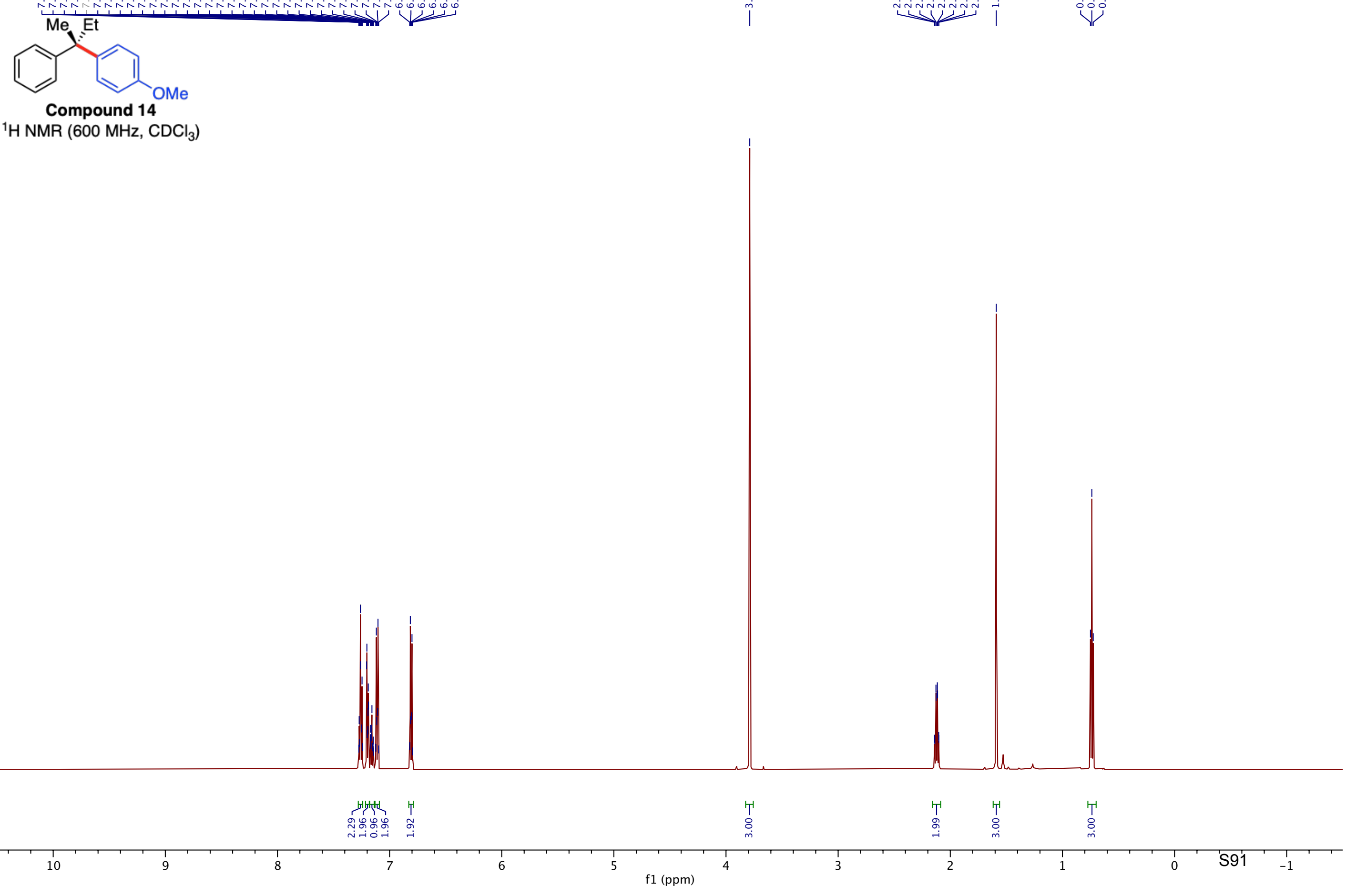
$\mathrm{Me}$ Et

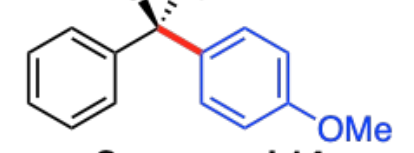

Compound 14

${ }^{13} \mathrm{C}$ NMR (151 MHz, $\mathrm{CDCl}_{3}$ )

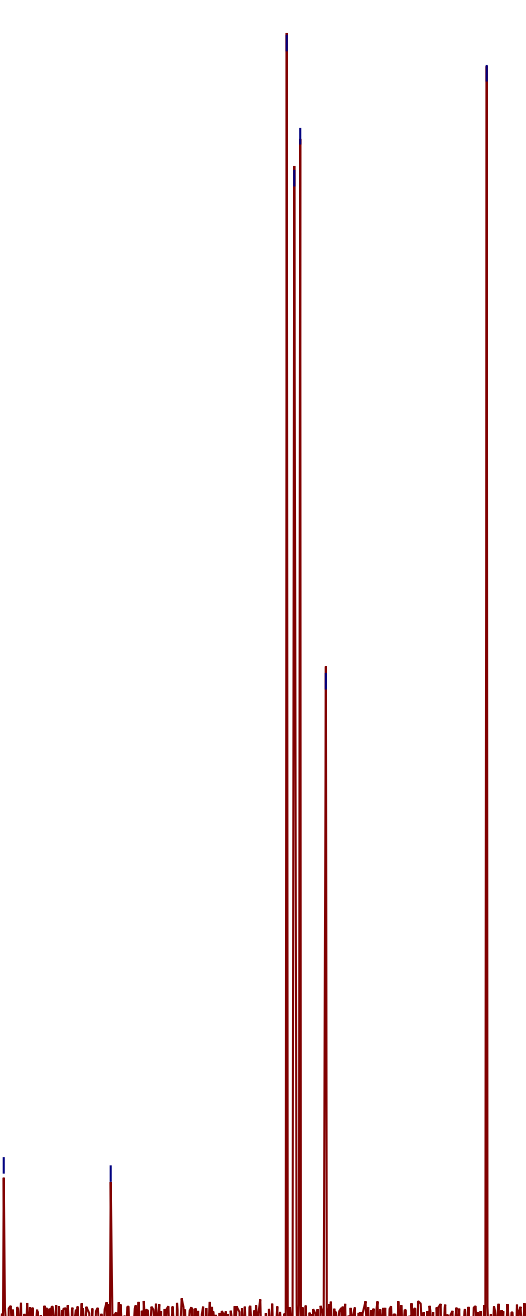




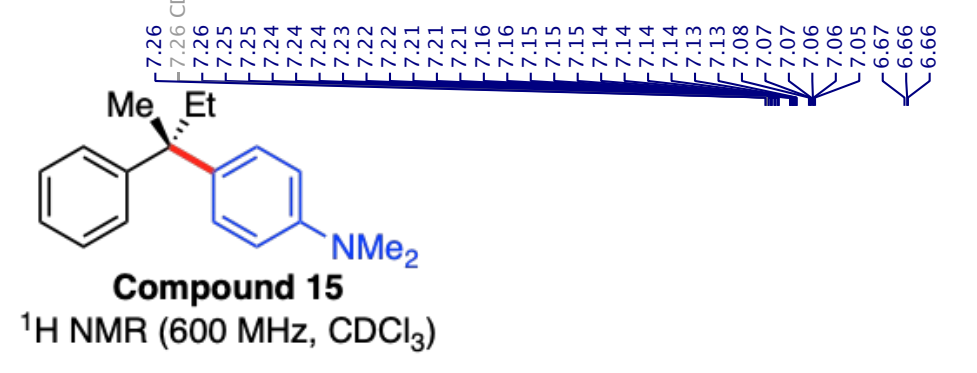

iू

${ }^{1} \mathrm{H}$ NMR $\left(600 \mathrm{MHz}, \mathrm{CDCl}_{3}\right)$ 


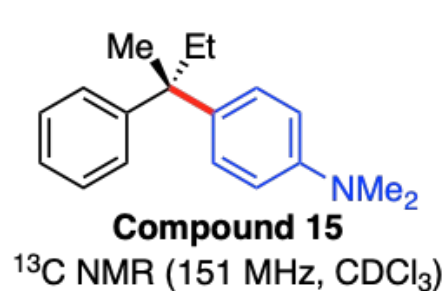

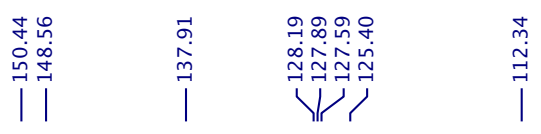

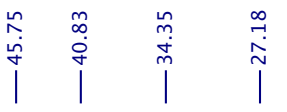

${ }^{13} \mathrm{C}$ NMR (151 MHz, $\mathrm{CDCl}_{3}$ )

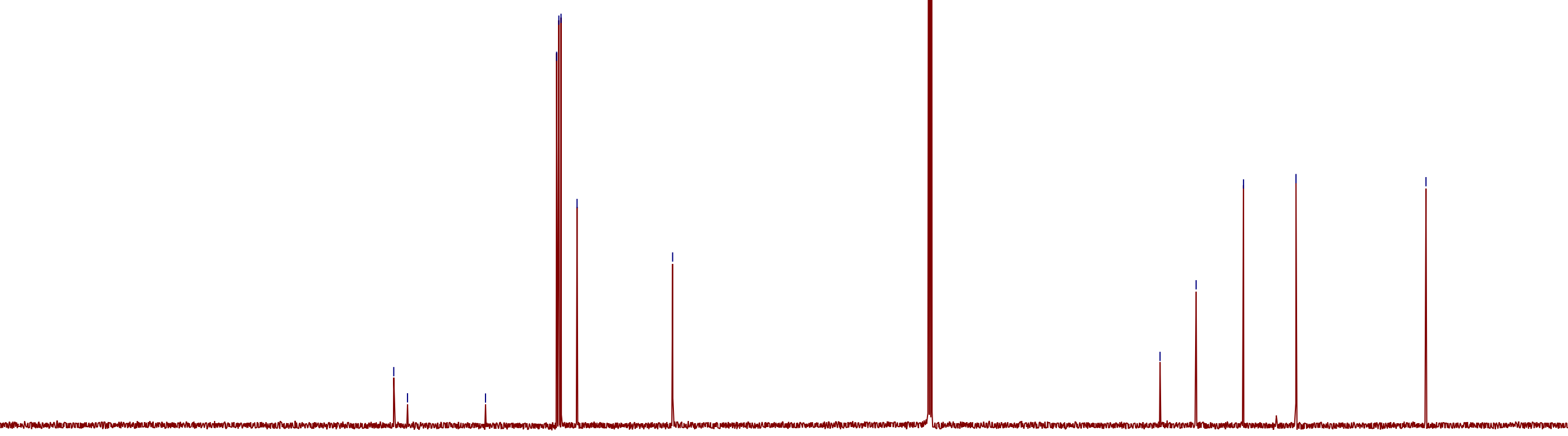




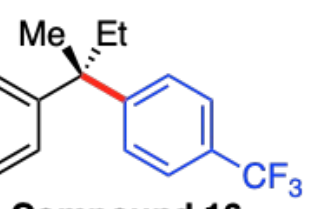

Compound 16

${ }^{1} \mathrm{H}$ NMR $\left(600 \mathrm{MHz}, \mathrm{CDCl}_{3}\right)$

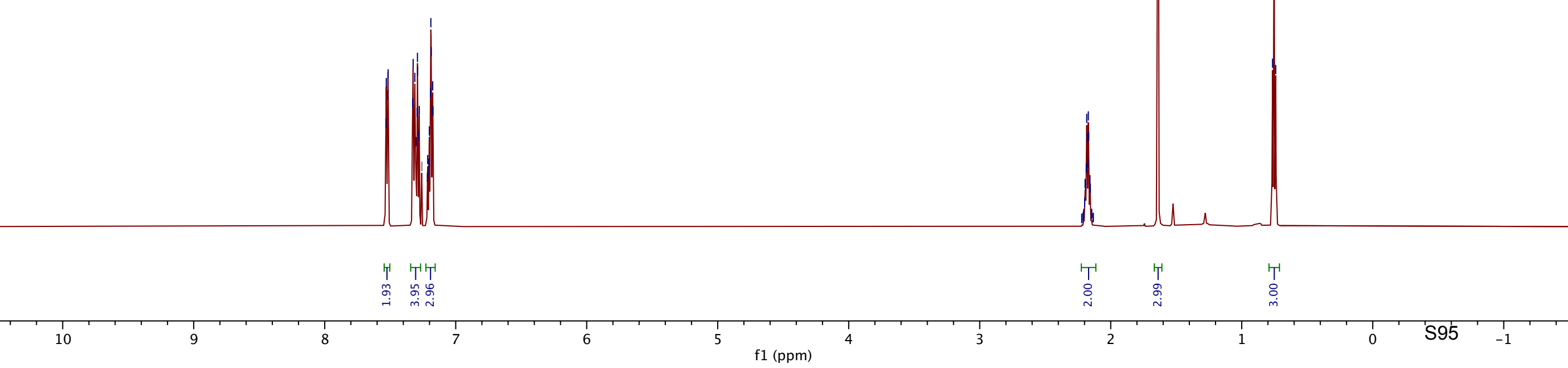




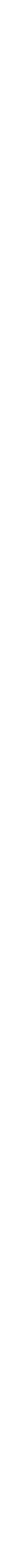



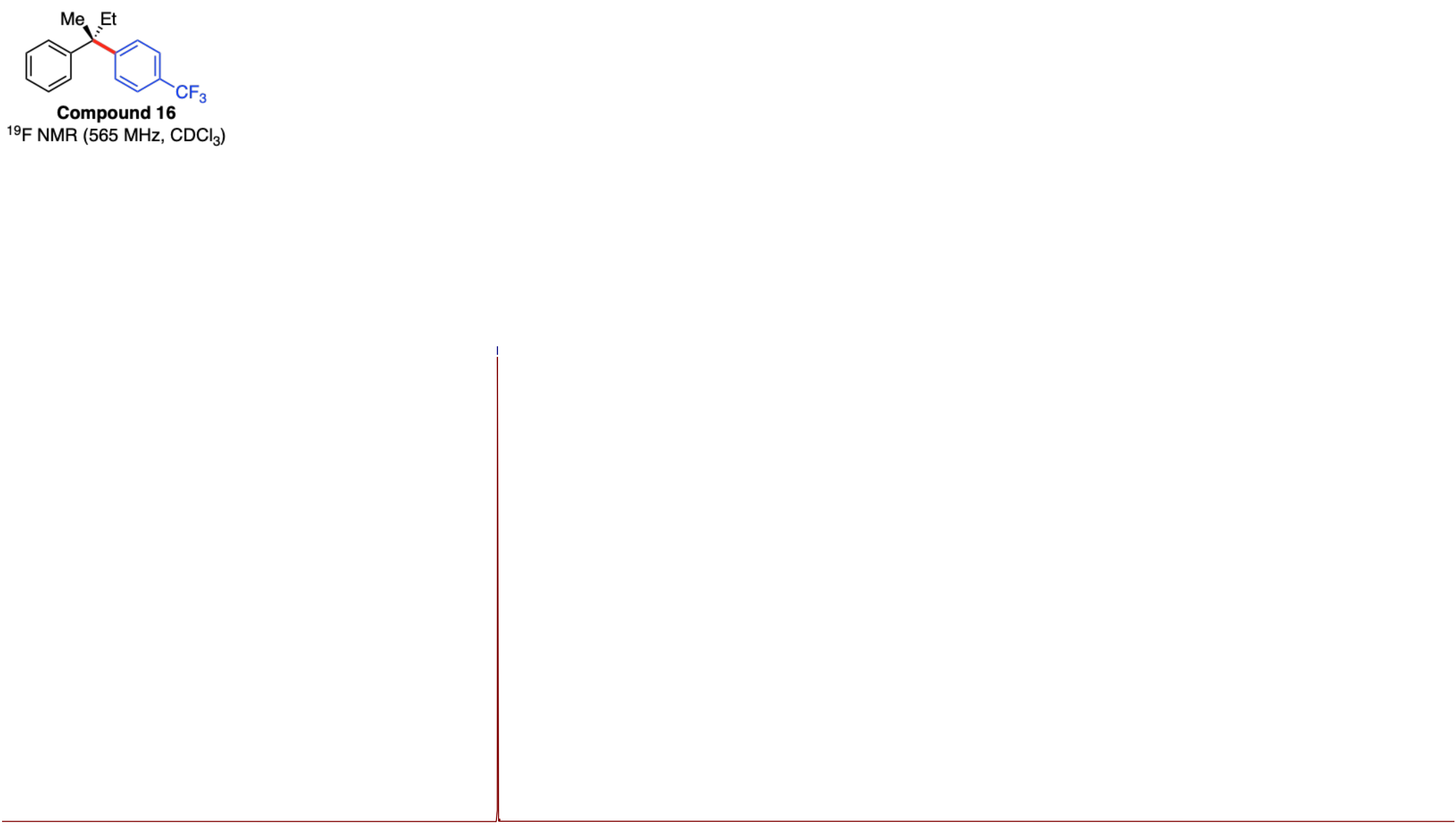


\title{
$\mathrm{Me}, \mathrm{Et}$
}
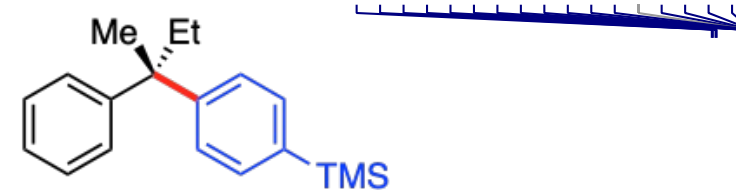

Compound 17

${ }^{1} \mathrm{H}$ NMR $\left(600 \mathrm{MHz}, \mathrm{CDCl}_{3}\right)$

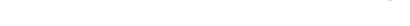 \\ .
}



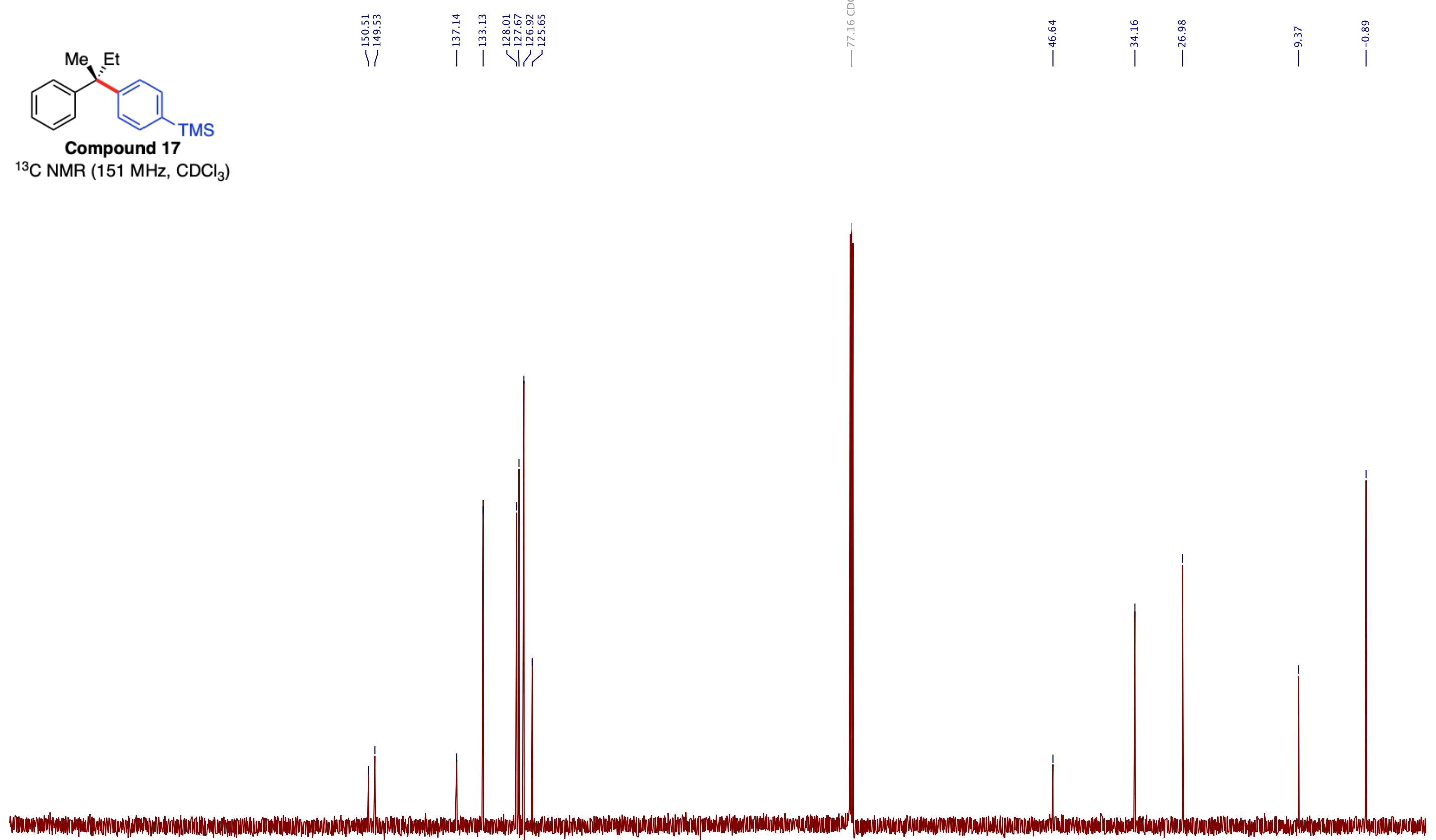

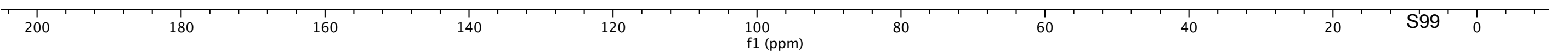




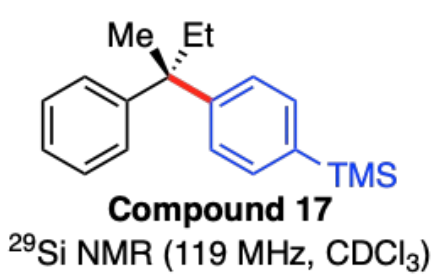

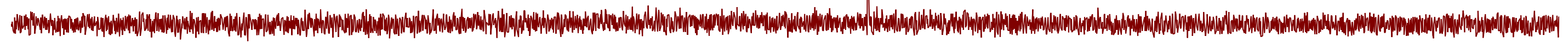


$\mathrm{Me}$, Et

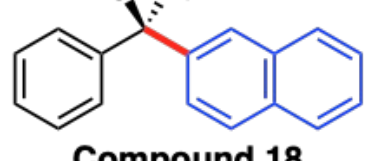

Compound 18

${ }^{1} \mathrm{H}$ NMR $\left(600 \mathrm{MHz}, \mathrm{CDCl}_{3}\right)$

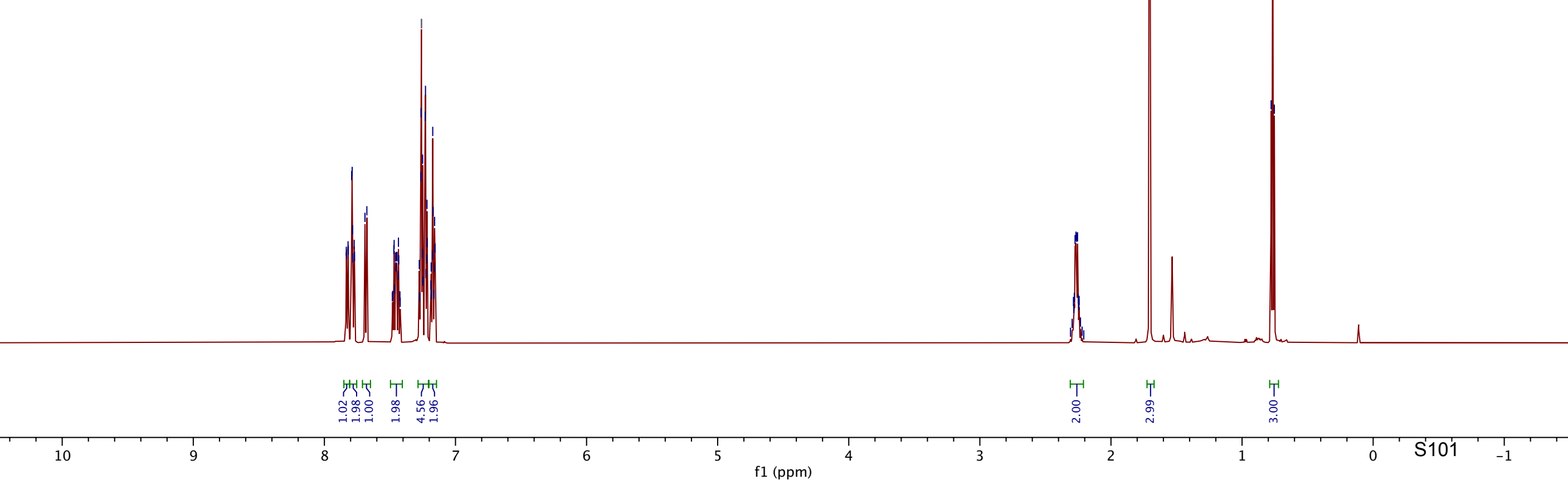




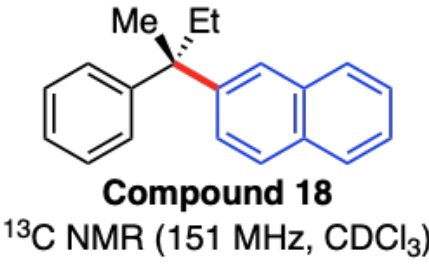

${ }^{13} \mathrm{C}$ NMR (151 MHz, $\left.\mathrm{CDCl}_{3}\right)$

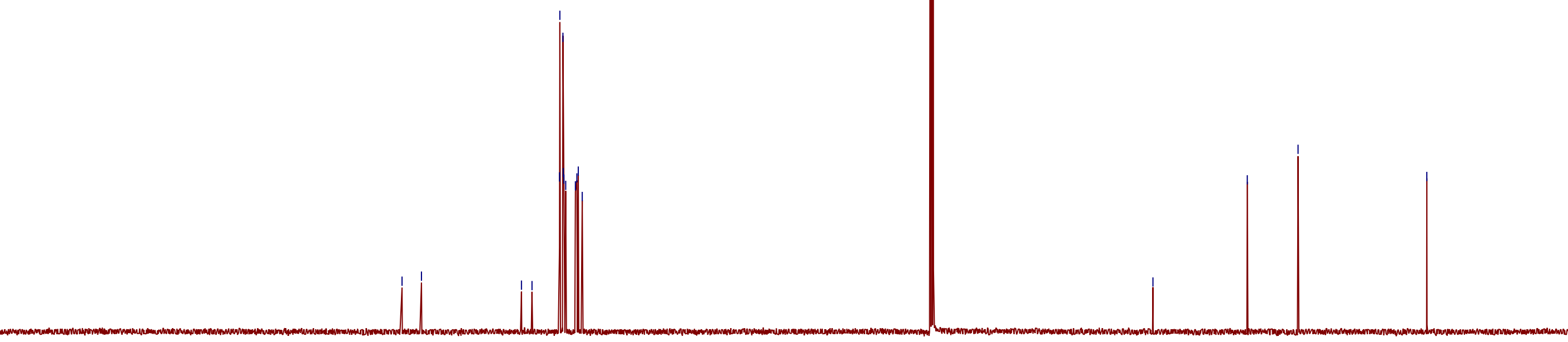




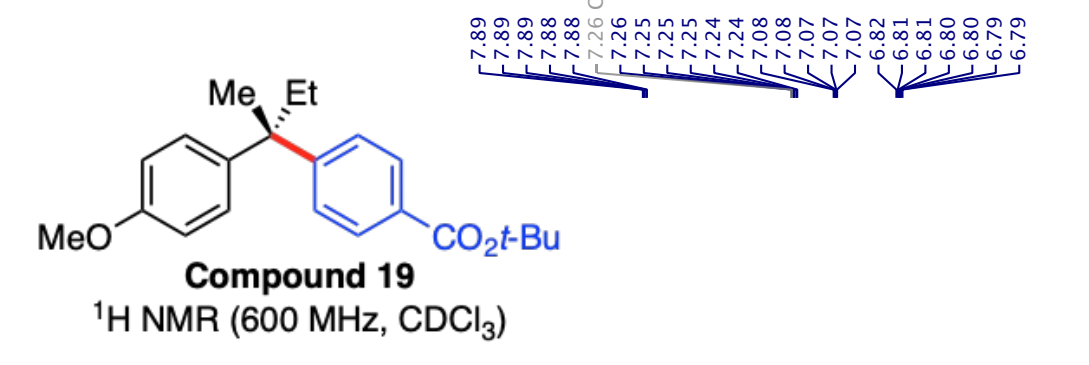

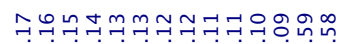

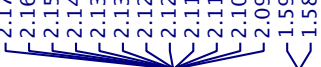

${ }^{1} \mathrm{H}$ NMR $\left(600 \mathrm{MHz}, \mathrm{CDCl}_{3}\right)$

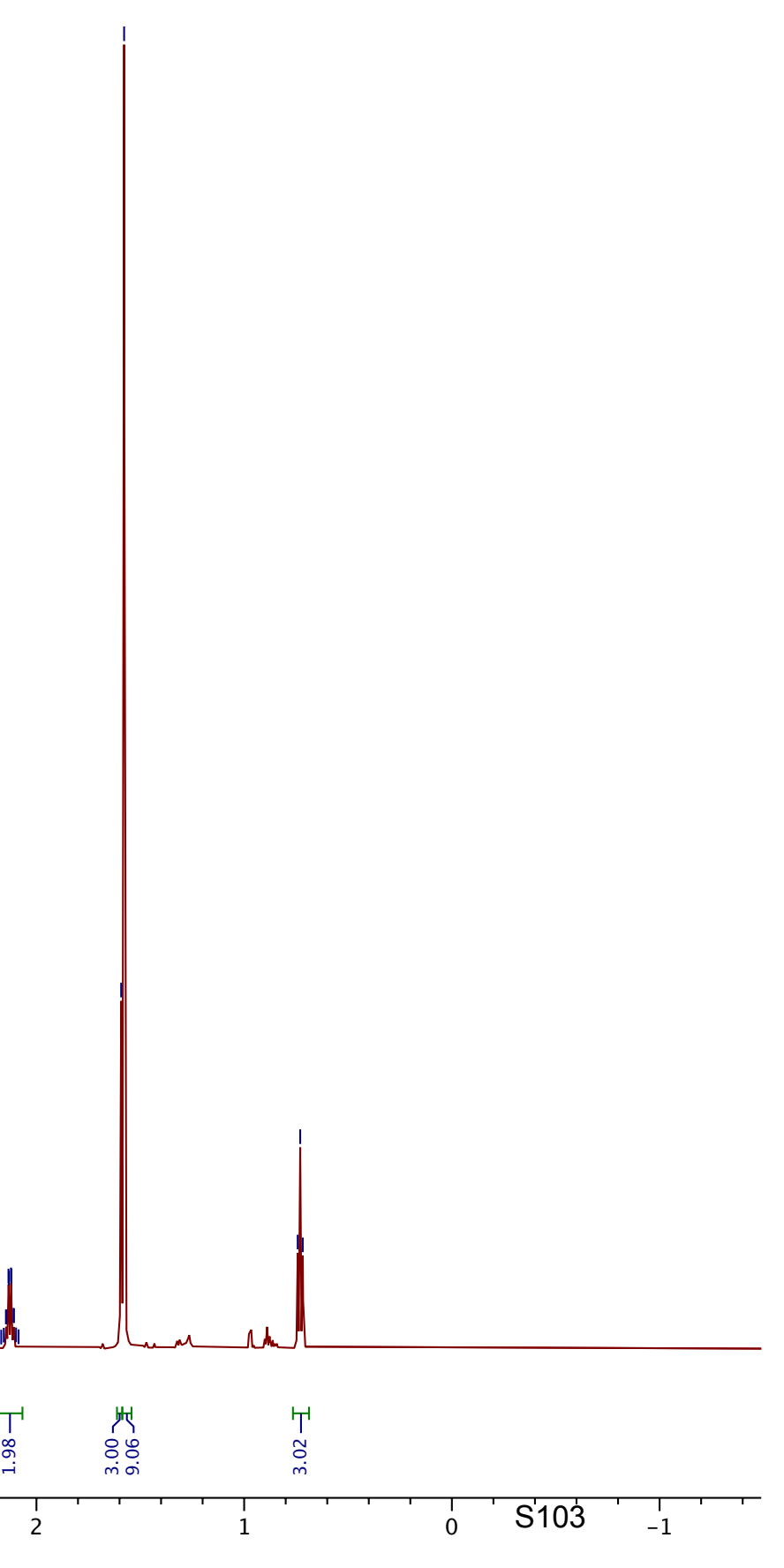




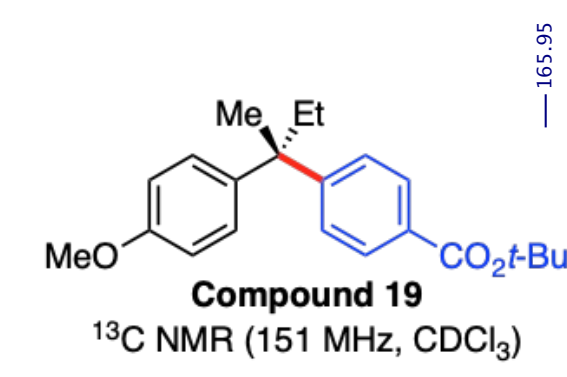

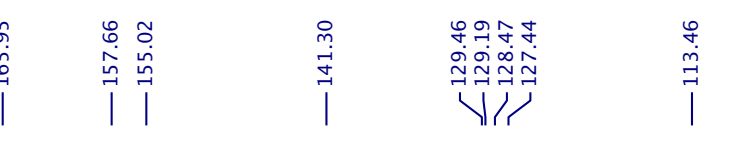

${ }^{13} \mathrm{C}$ NMR (151 MHz, $\mathrm{CDCl}_{3}$ )

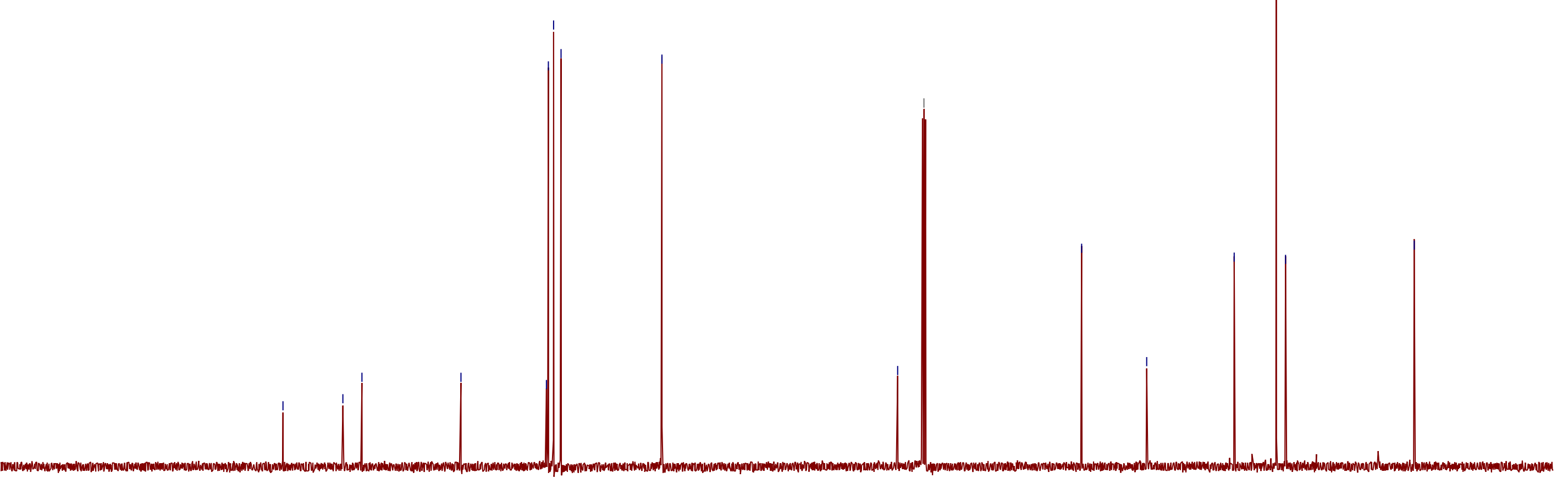




$$
\mathrm{Me} \text { Et }
$$<smiles>CC(C)(c1ccccc1)c1cccc(OCc2ccccc2)c1</smiles>

\section{Compound 20}

${ }^{1} \mathrm{H}$ NMR $\left(600 \mathrm{MHz}, \mathrm{CDCl}_{3}\right)$

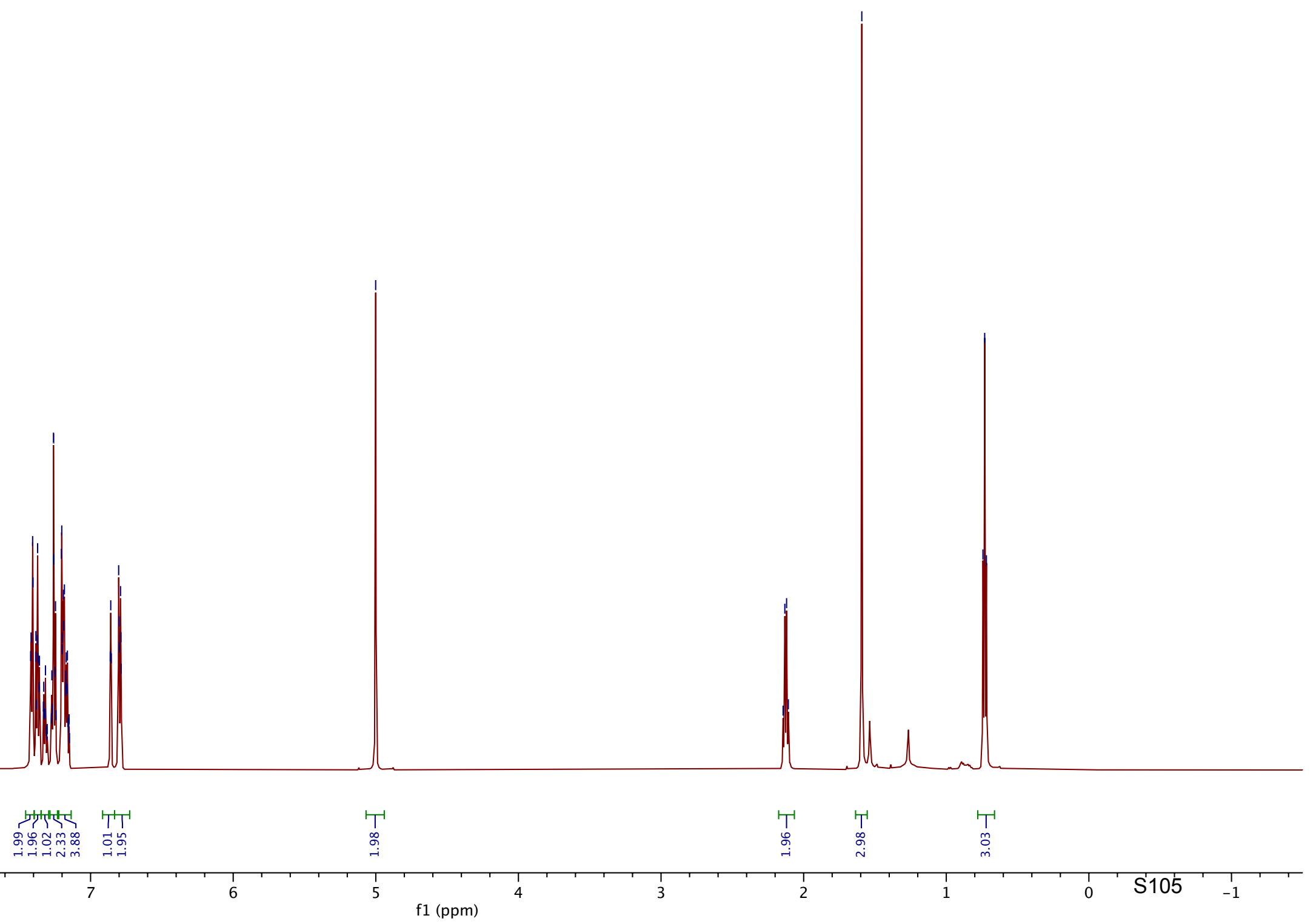



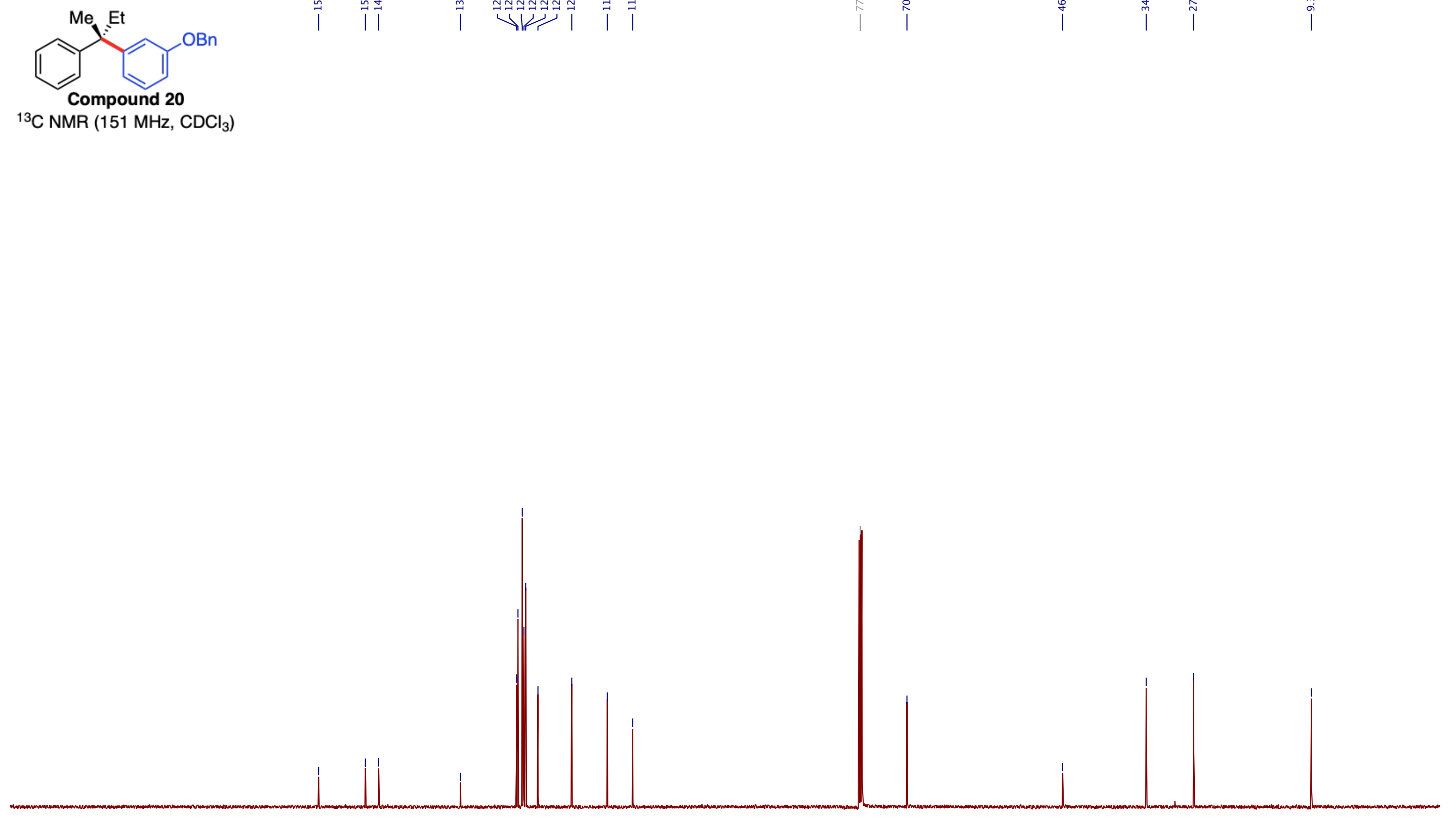


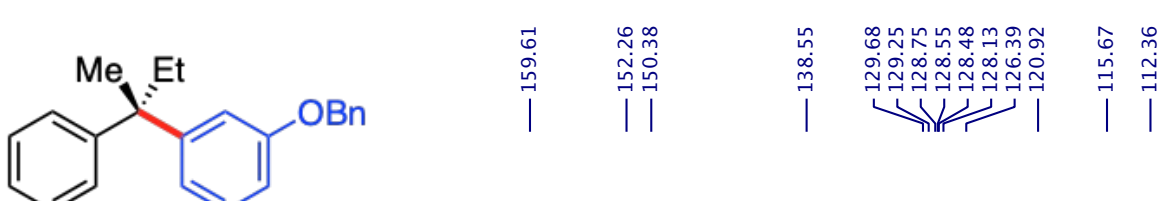

Compound 20

${ }^{13} \mathrm{C}$ NMR $\left(151 \mathrm{MHz},\left(\mathrm{CD}_{3}\right)_{2} \mathrm{CO}\right)$

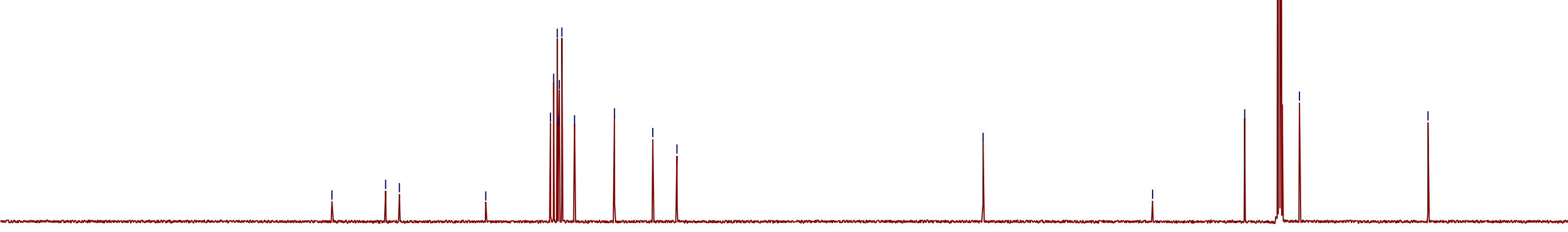




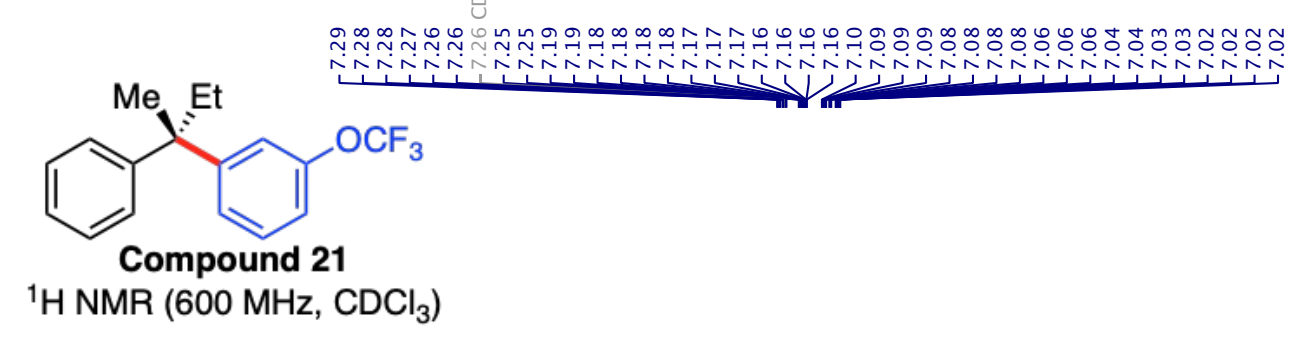

${ }^{1} \mathrm{H} \mathrm{NMR}\left(600 \mathrm{MHz}, \mathrm{CDCl}_{3}\right)$

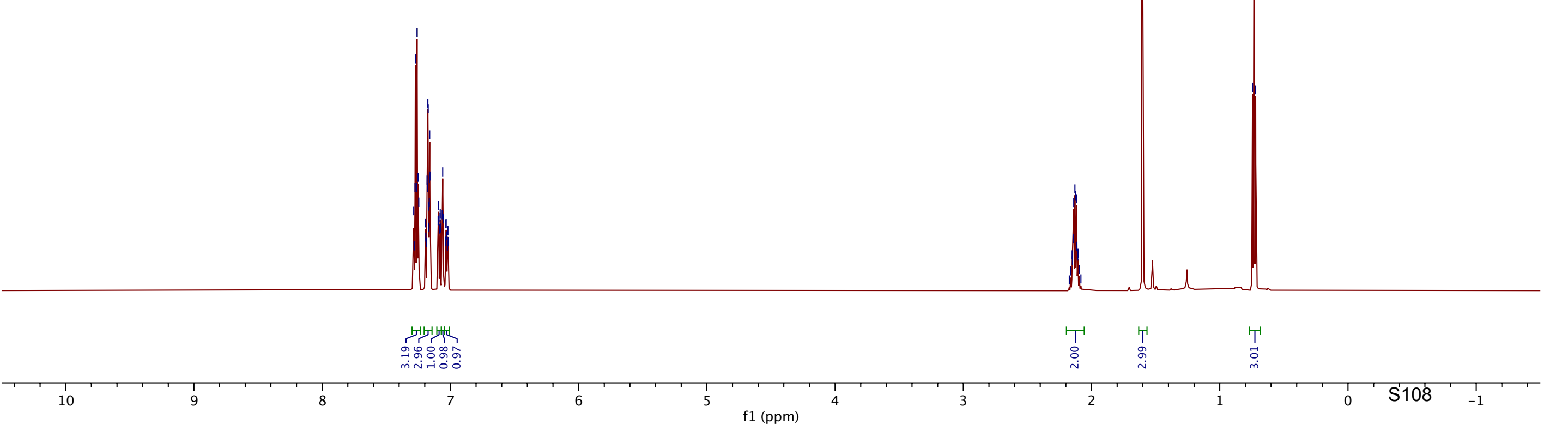



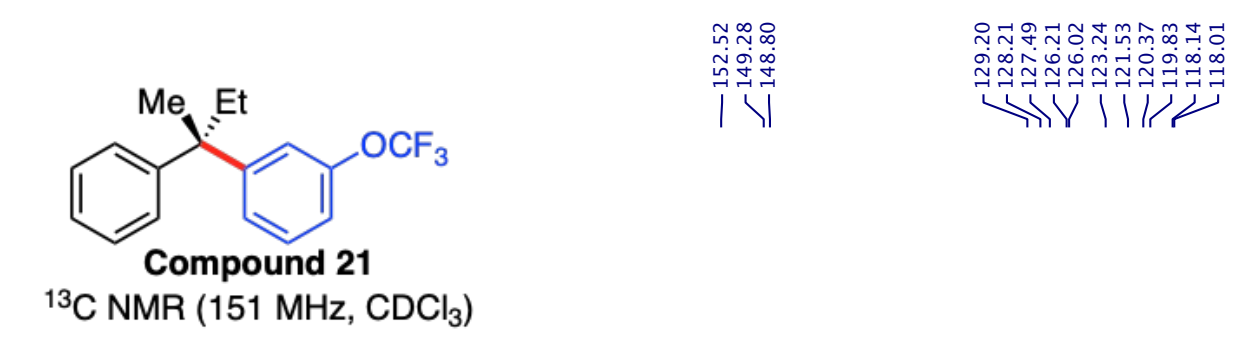

${ }^{13} \mathrm{C}$ NMR (151 MHz, $\mathrm{CDCl}_{3}$ )

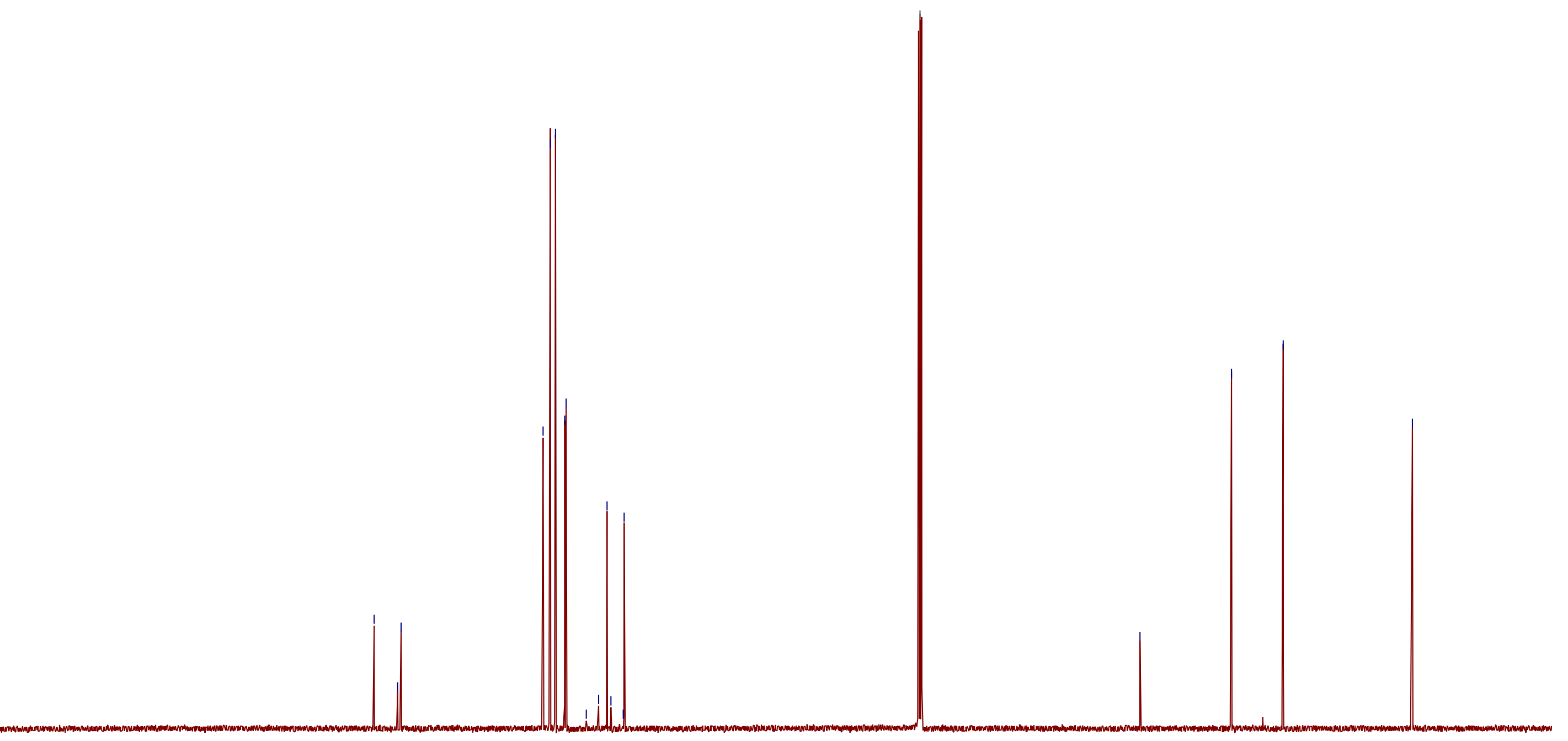

\begin{tabular}{|c|c|c|c|c|c|c|c|c|c|c|}
\hline 200 & 180 & 160 & $\begin{array}{l}1 \\
140\end{array}$ & 120 & $\begin{array}{l}100 \\
\text { f1 (ppm) }\end{array}$ & 80 & 60 & 40 & 20 & S109' \\
\hline
\end{tabular}


Compound 21

${ }^{19} \mathrm{~F} \mathrm{NMR}\left(565 \mathrm{MHz}, \mathrm{CDCl}_{3}\right.$ )

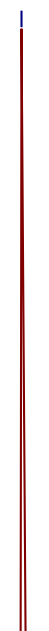




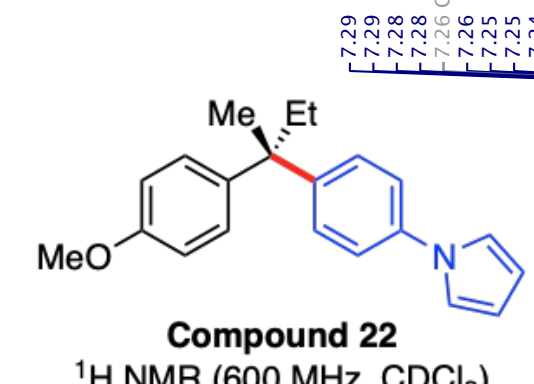

${ }^{1} \mathrm{H}$ NMR (600 MHz, $\mathrm{CDCl}_{3}$ )

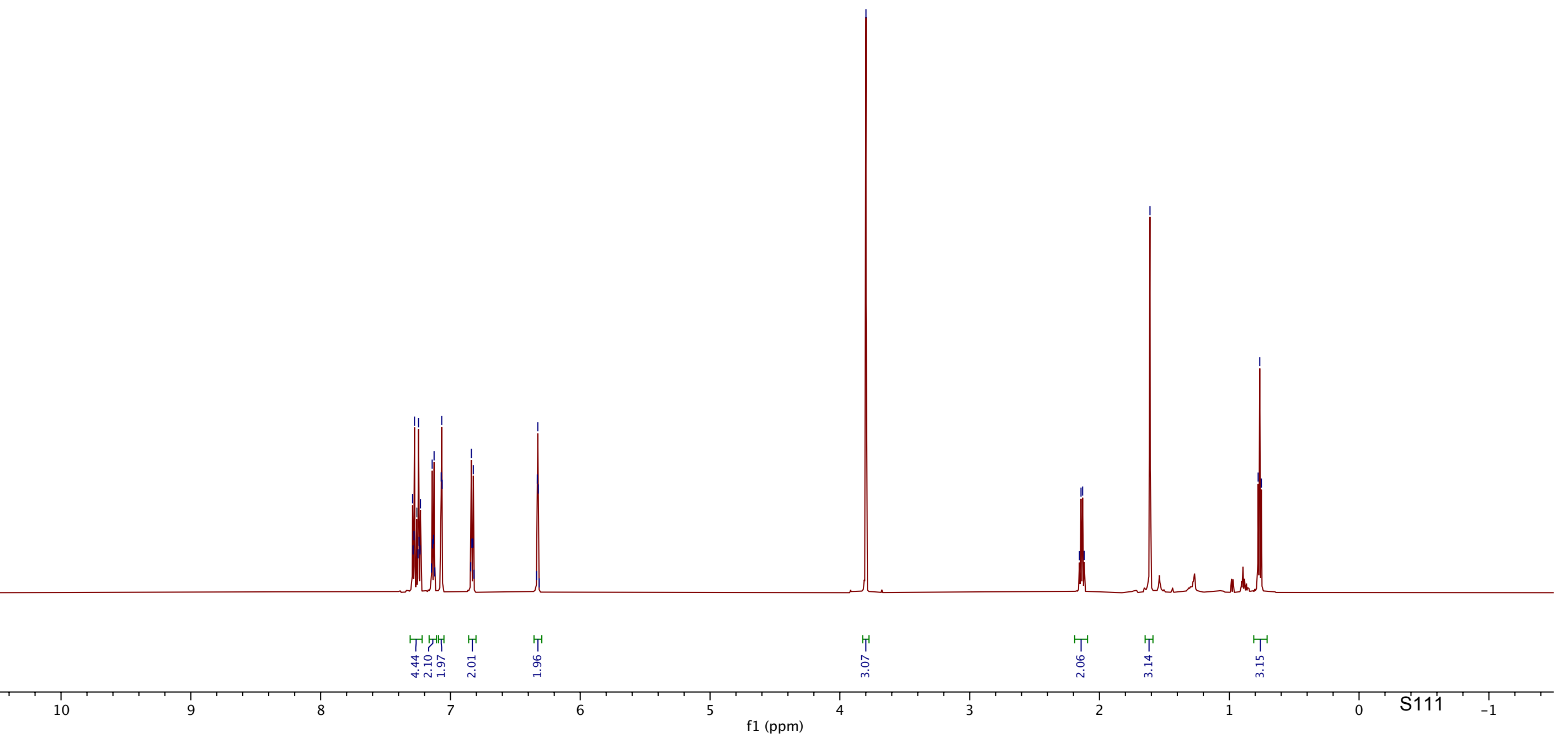




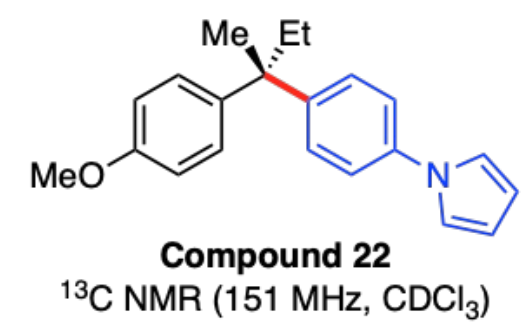

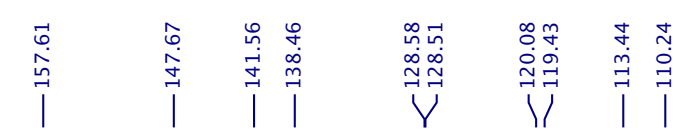

${ }^{13} \mathrm{C}$ NMR (151 MHz, $\mathrm{CDCl}_{3}$ ) 


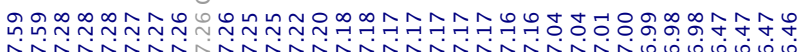
$\mathrm{Me}$ Et

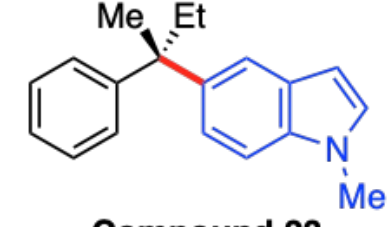

Compound 23

${ }^{1} \mathrm{H}$ NMR $\left(600 \mathrm{MHz}, \mathrm{CDCl}_{3}\right)$

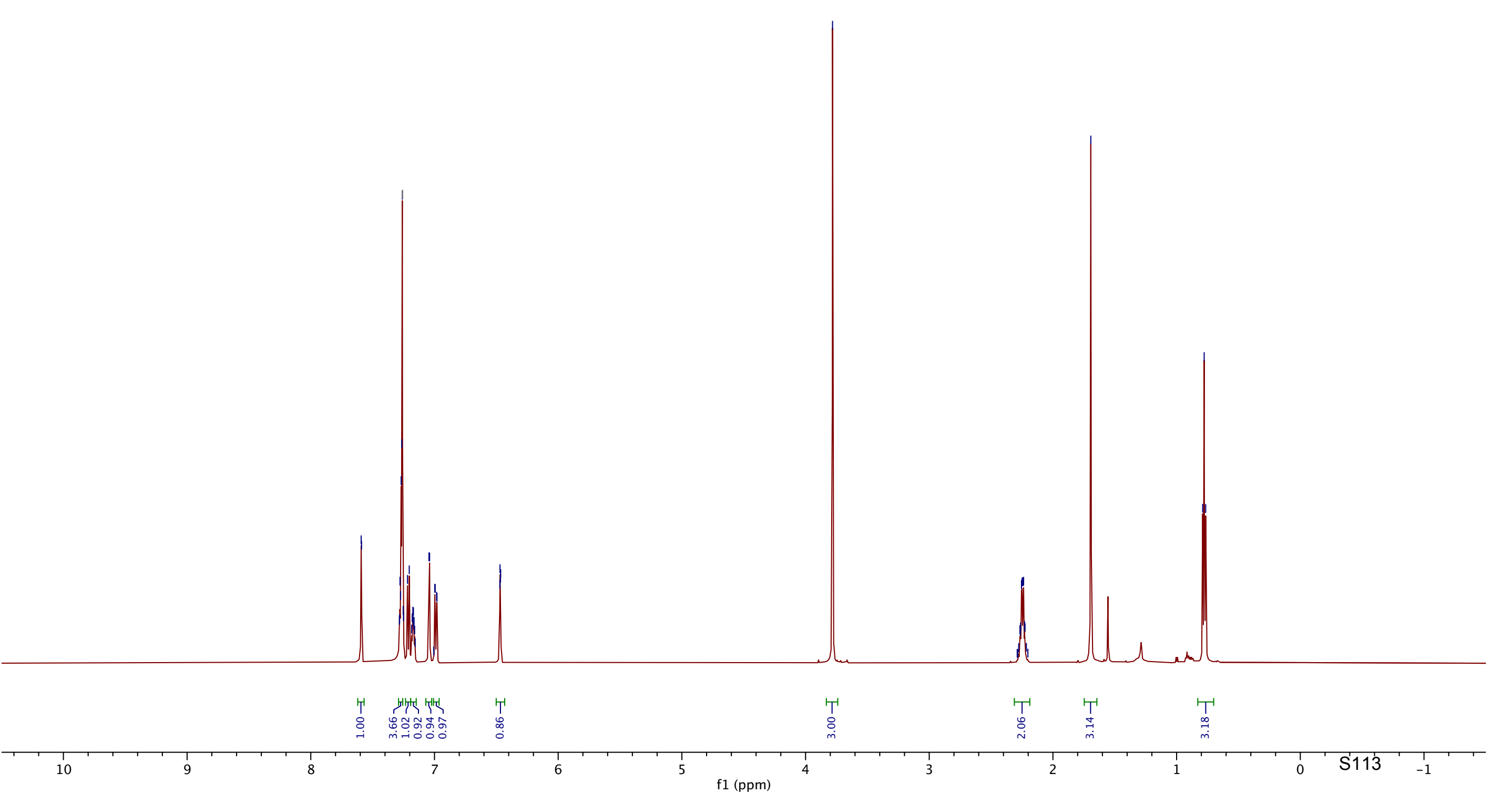




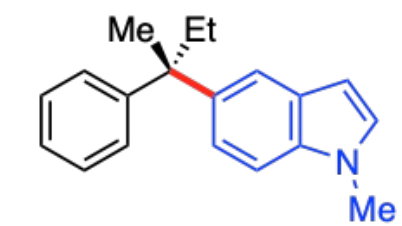

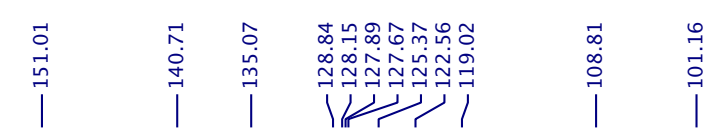

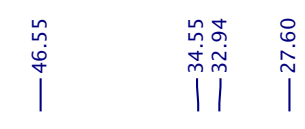

Compound 23

${ }^{13} \mathrm{C}$ NMR $\left(151 \mathrm{MHz}, \mathrm{CDCl}_{3}\right)$

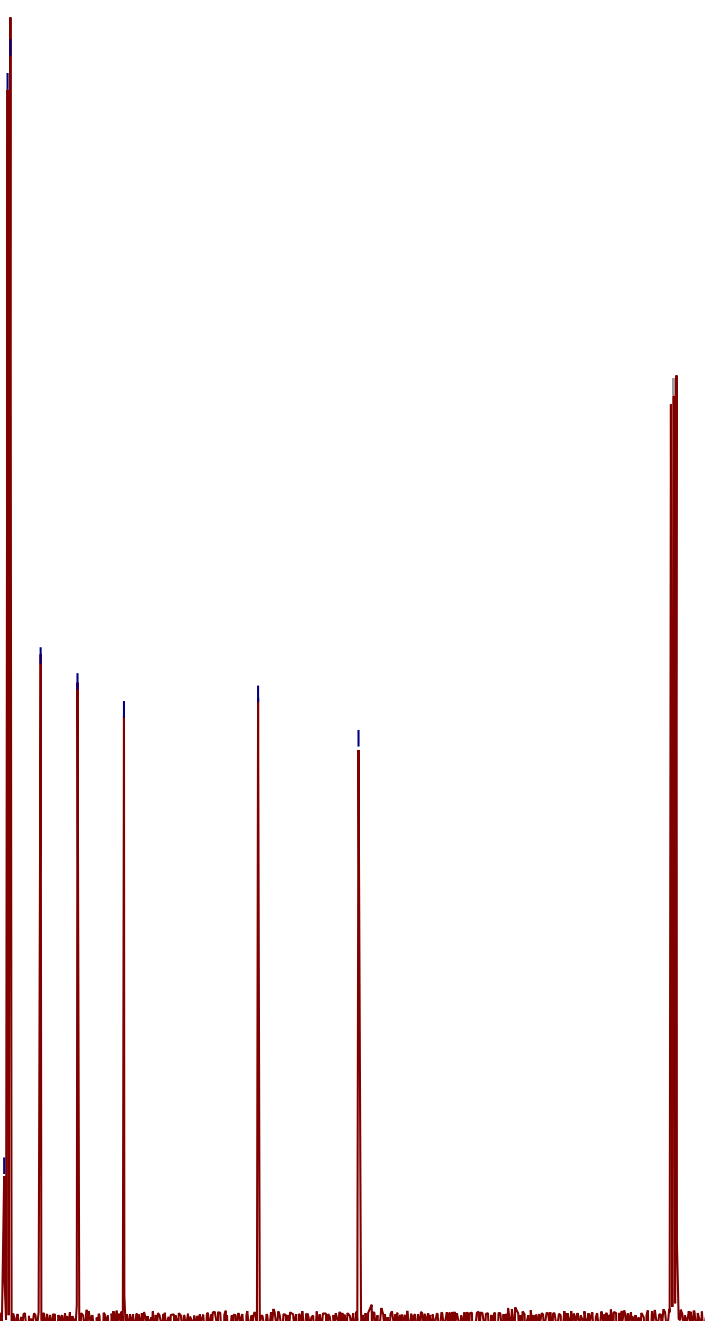

Mint

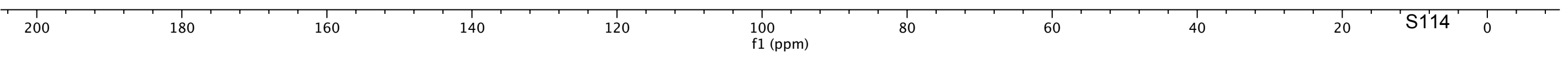




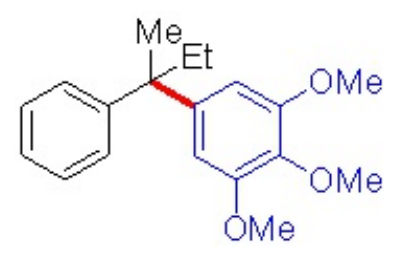

\section{Compound 24}

${ }^{1} \mathrm{H}$ NMR $\left(400 \mathrm{MHz}, \mathrm{CDCl}_{3}\right)$

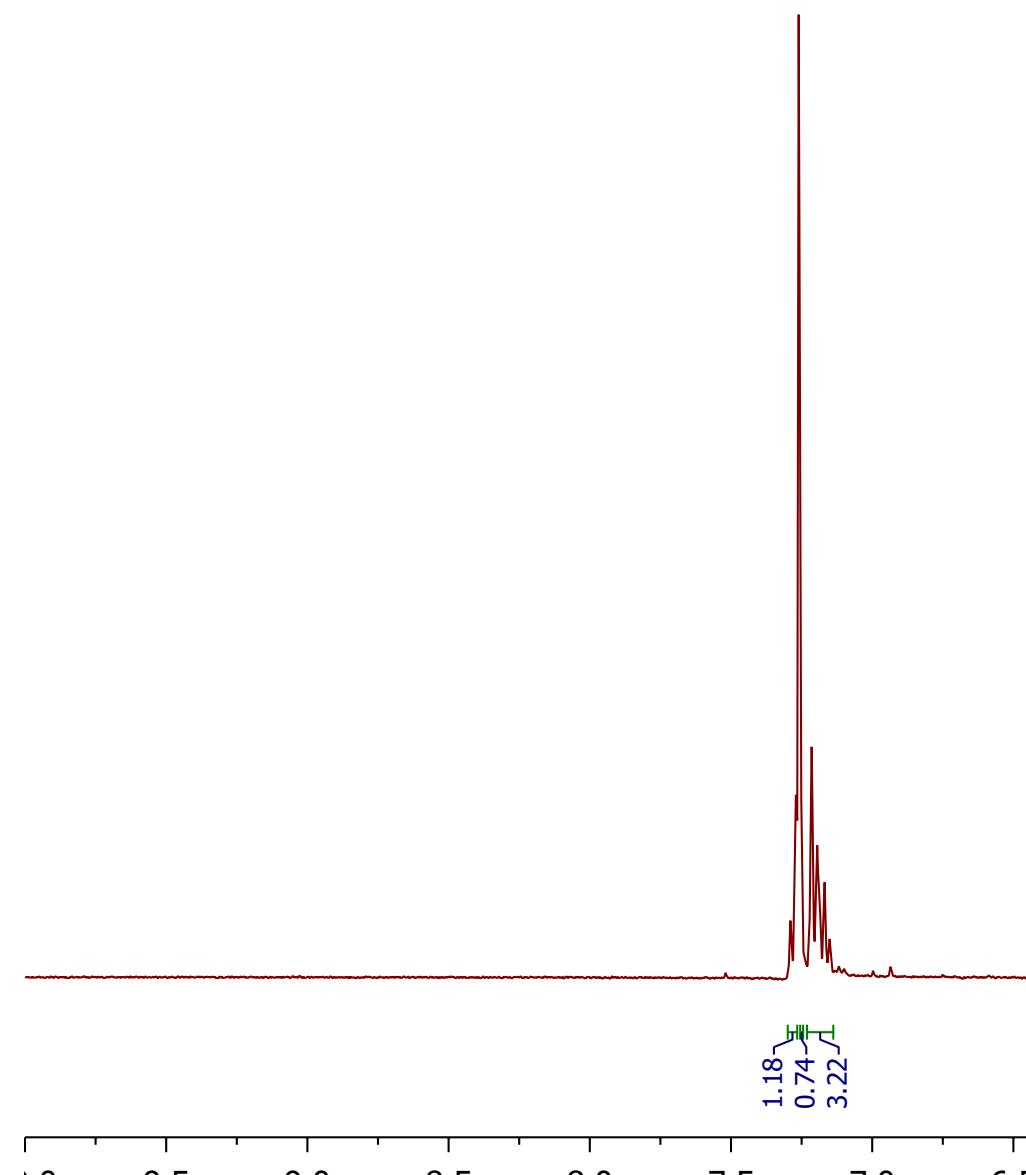

1.0 


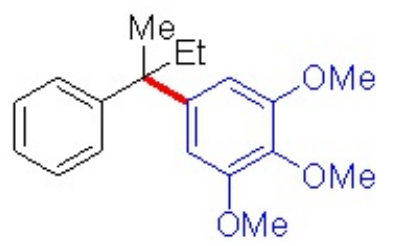

Compound 24

${ }^{13} \mathrm{C}$ NMR $\left(101 \mathrm{MHz}, \mathrm{CDCl}_{3}\right)$
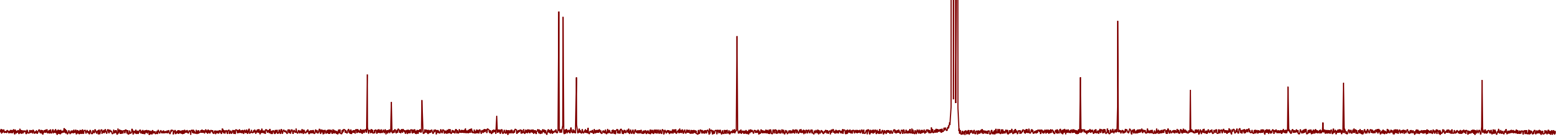


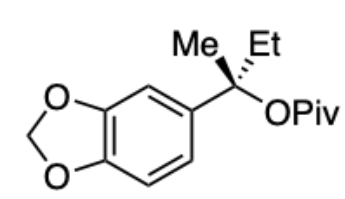

Compound $1 \mathrm{c}$

${ }^{1} \mathrm{H}$ NMR $\left(600 \mathrm{MHz}, \mathrm{CDCl}_{3}\right.$ )

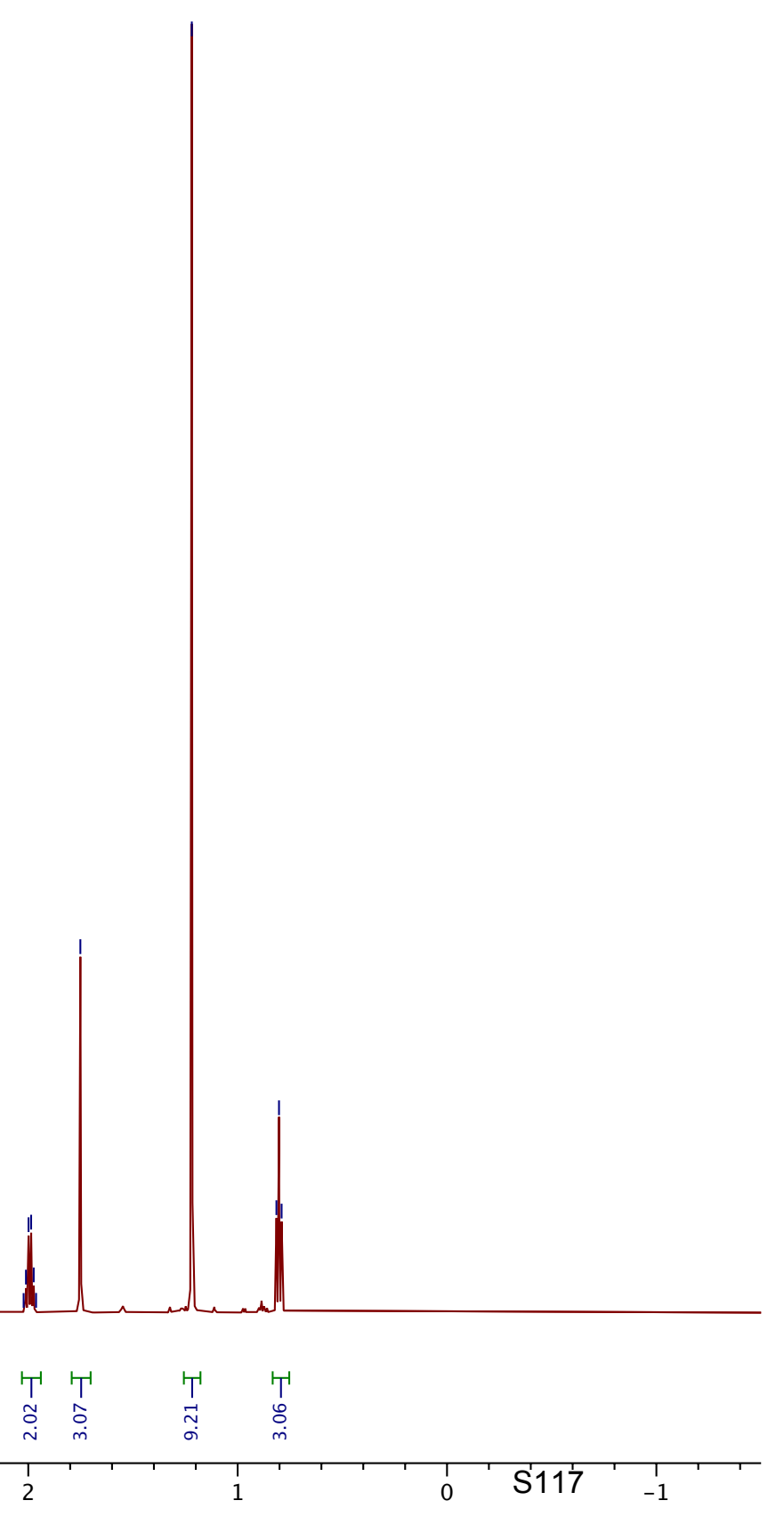




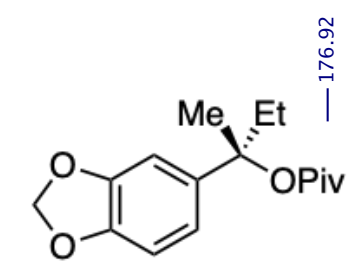

Compound 1c

${ }^{13} \mathrm{C}$ NMR (151 MHz, $\mathrm{CDCl}_{3}$ )

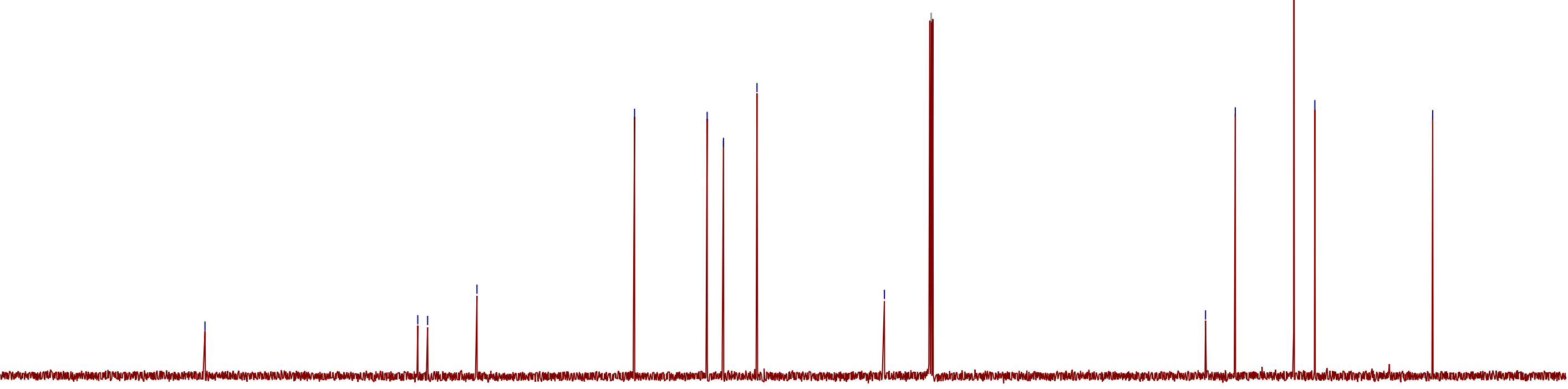




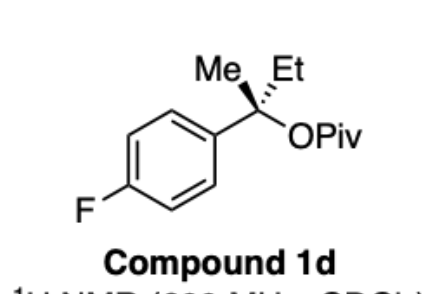

Compound 1d

${ }^{1} \mathrm{H}$ NMR $\left(600 \mathrm{MHz}, \mathrm{CDCl}_{3}\right)$

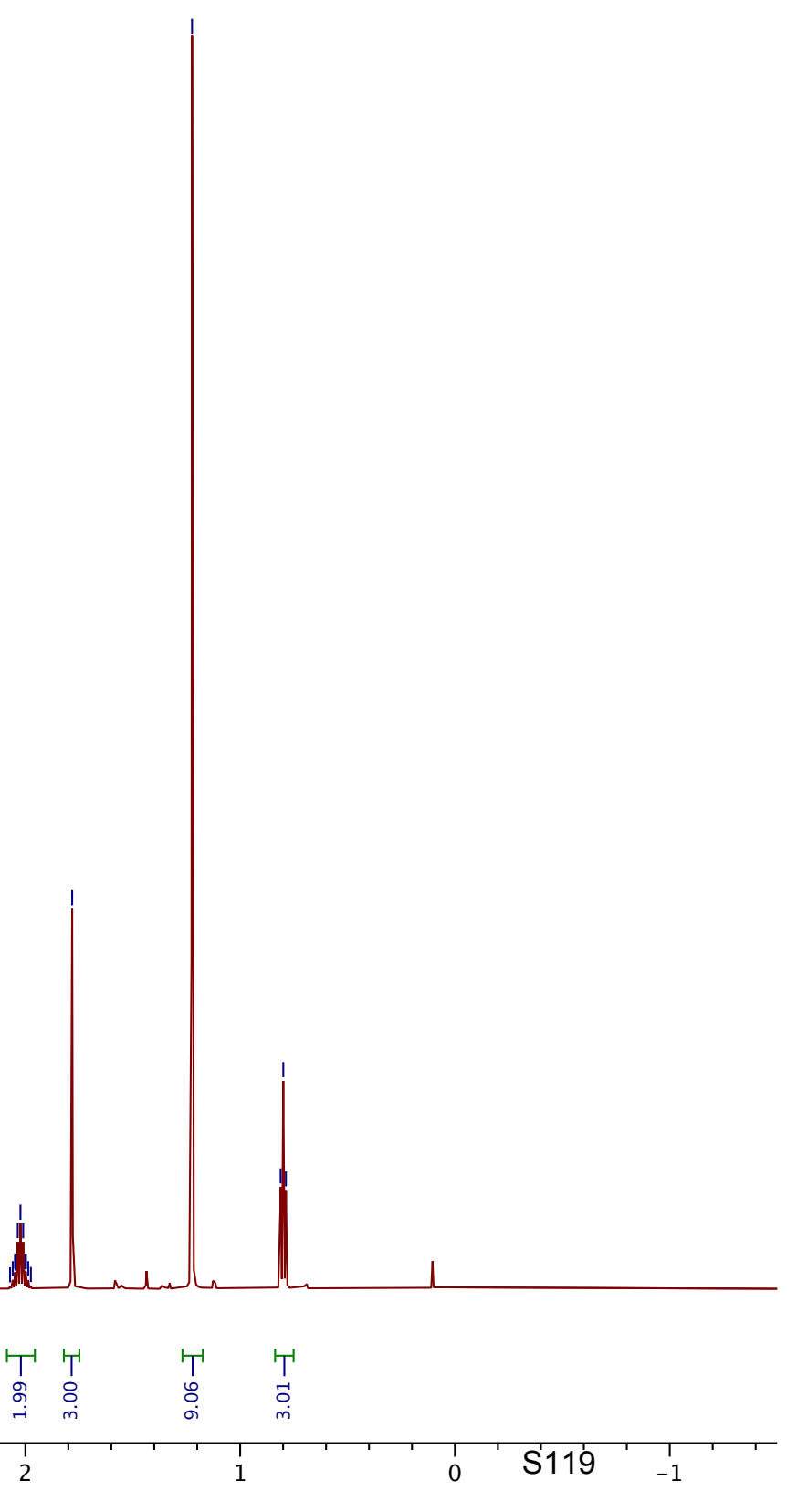




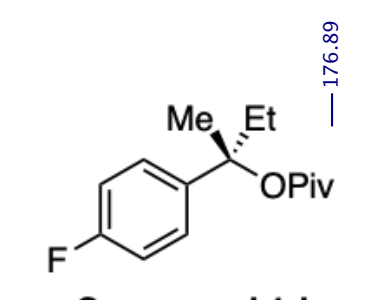

Compound 1d

${ }^{13} \mathrm{C}$ NMR (151 MHz, $\mathrm{CDCl}_{3}$ ) 
$\mathrm{Me}$ Et

\section{Compound 1d}

${ }^{19} \mathrm{~F}$ NMR (565 MHz, $\mathrm{CDCl}_{3}$ )

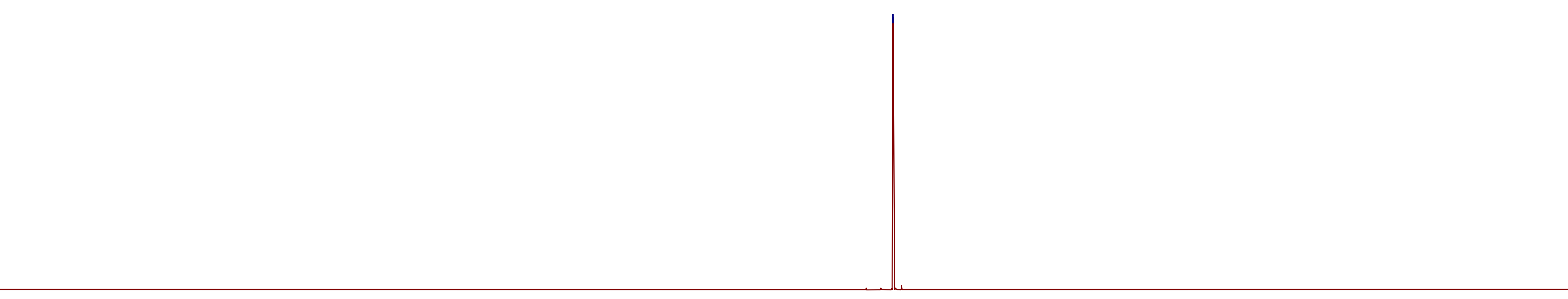




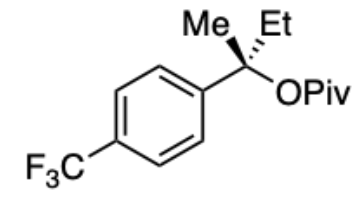

\section{Compound $1 \mathrm{e}$}

${ }^{1} \mathrm{H}$ NMR $\left(600 \mathrm{MHz}, \mathrm{CDCl}_{3}\right)$

\section{'Herch}

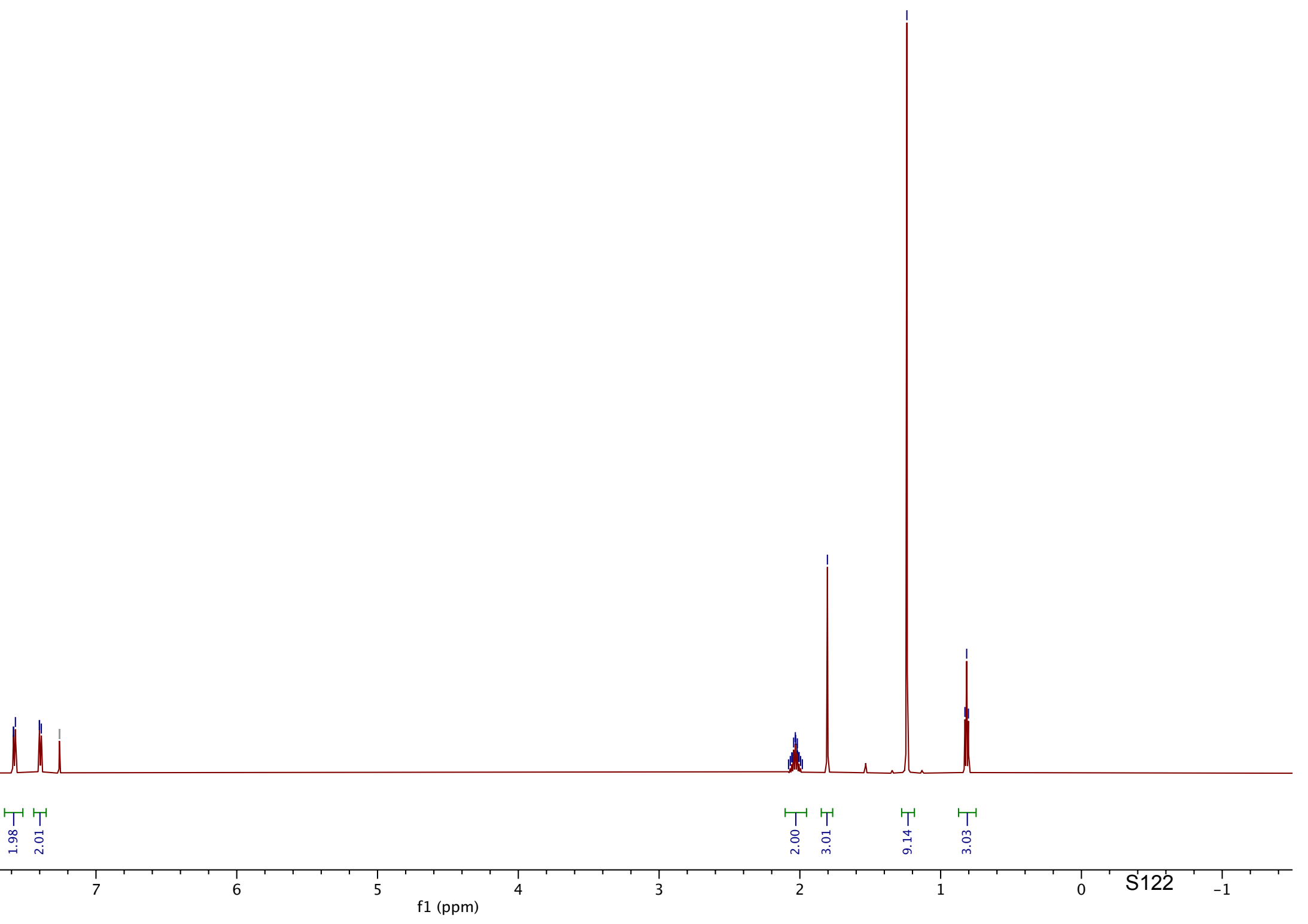




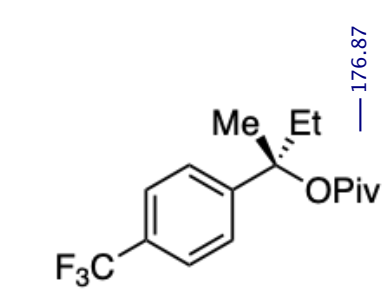

Compound 1e

${ }^{13} \mathrm{C}$ NMR (151 MHz, $\mathrm{CDCl}_{3}$ )

II

iñ.

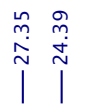

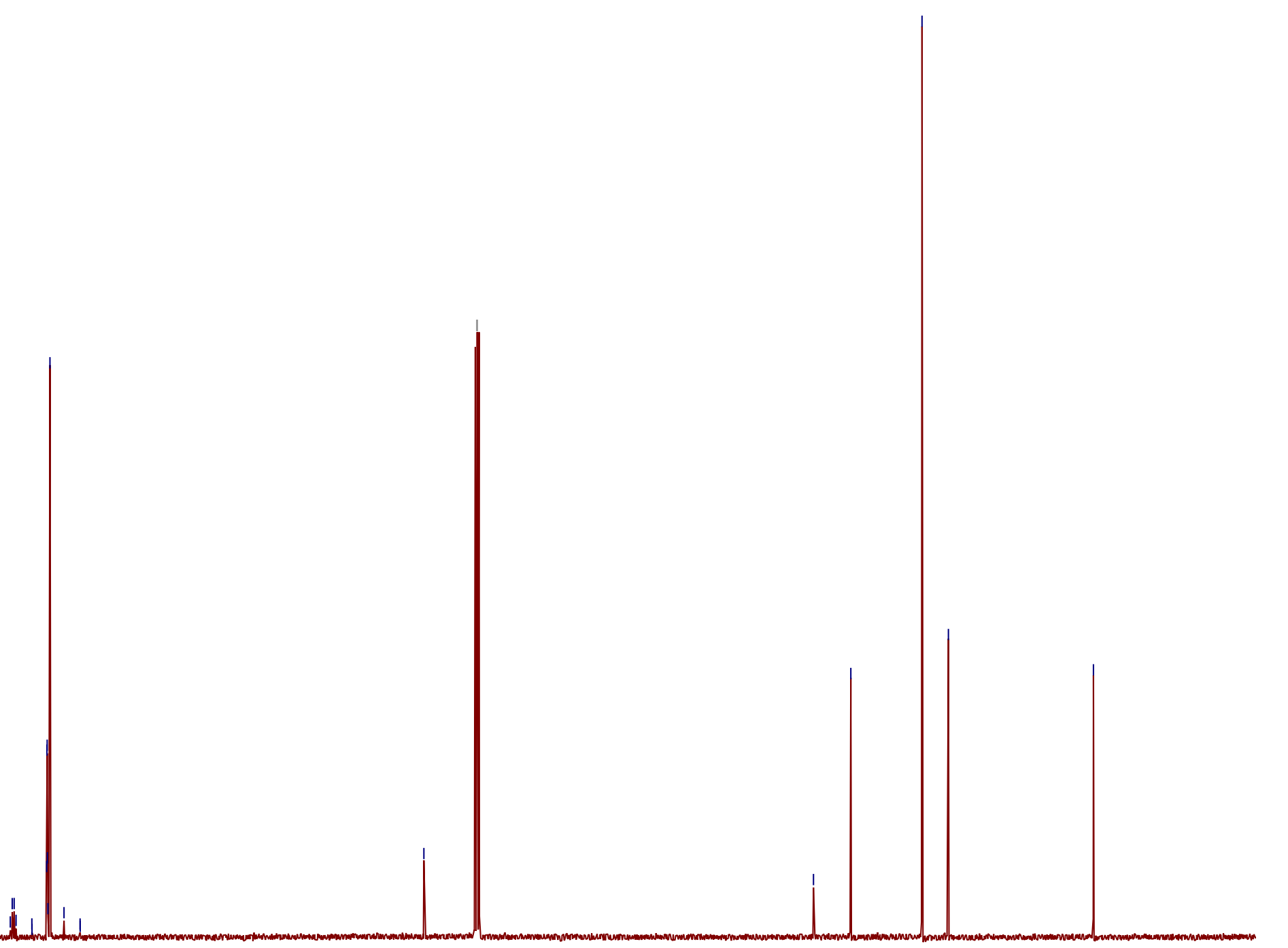




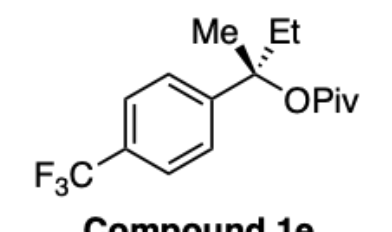

Compound 1e

${ }^{19} \mathrm{~F}$ NMR (565 MHz, $\mathrm{CDCl}_{3}$ )

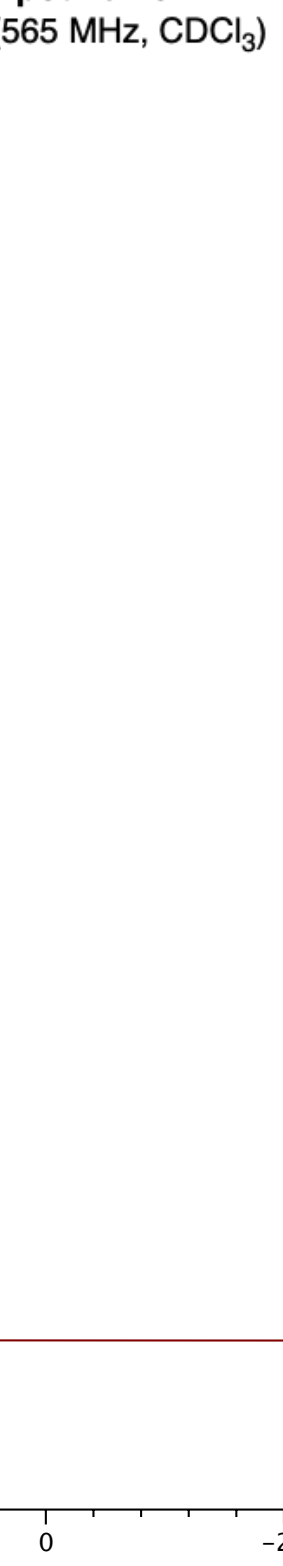

$-40$

$-60$

$-80$

$-100$

$-120$

$-140$

$-160$

$-180$

$\$ 1 z 40$ 
( 


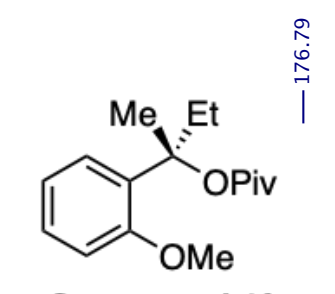

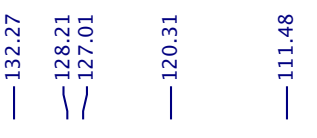

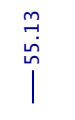

\section{Compound $1 f$}

${ }^{13} \mathrm{C}$ NMR (151 MHz, $\mathrm{CDCl}_{3}$ )

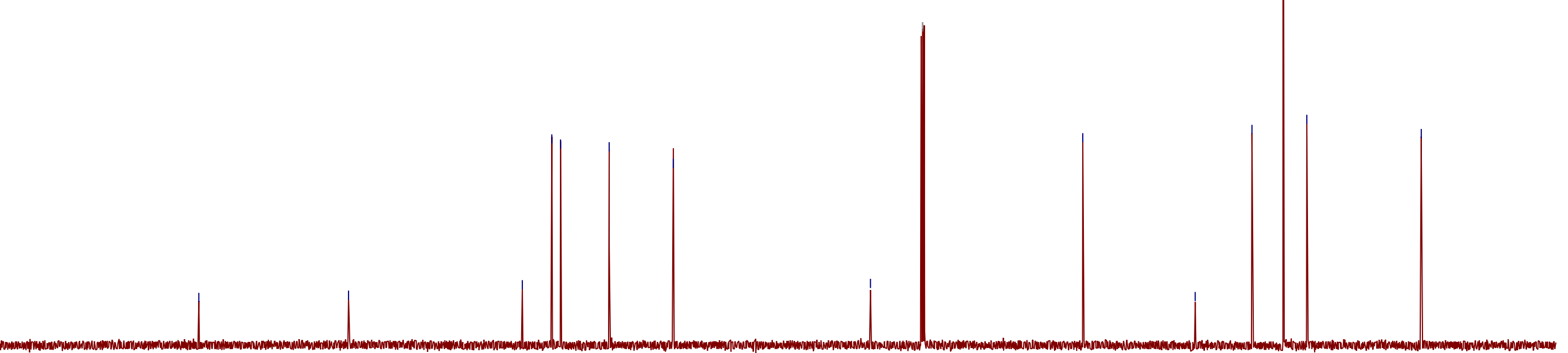




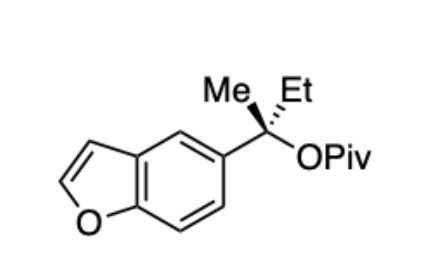

Compound 1h

${ }^{1} \mathrm{H}$ NMR $\left(600 \mathrm{MHz}, \mathrm{CDCl}_{3}\right)$

\section{'H}
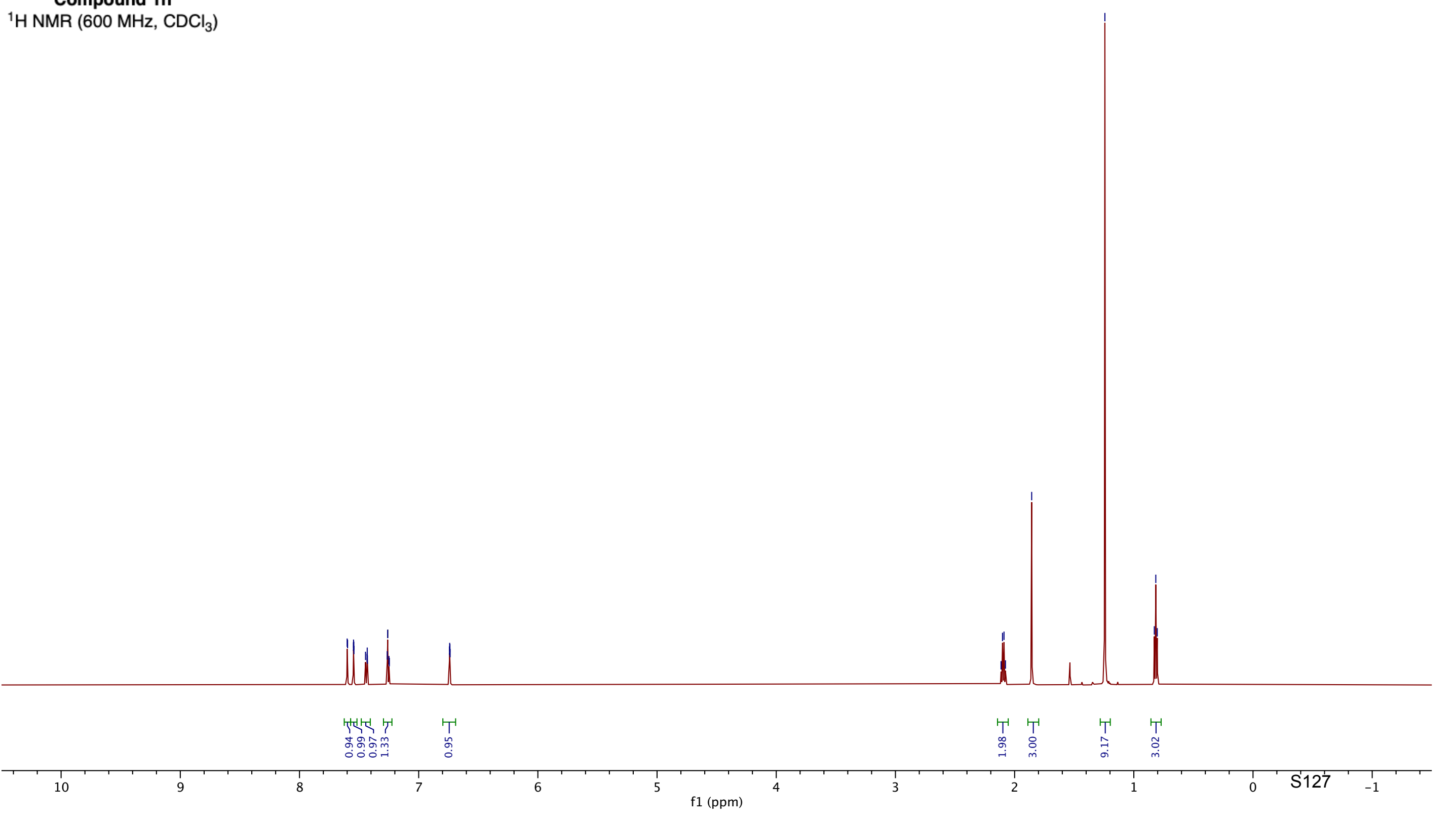


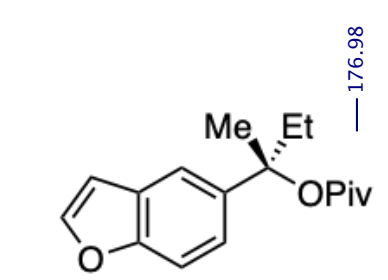

Compound $1 \mathrm{~h}$

${ }^{13} \mathrm{C}$ NMR (151 MHz, $\mathrm{CDCl}_{3}$ )

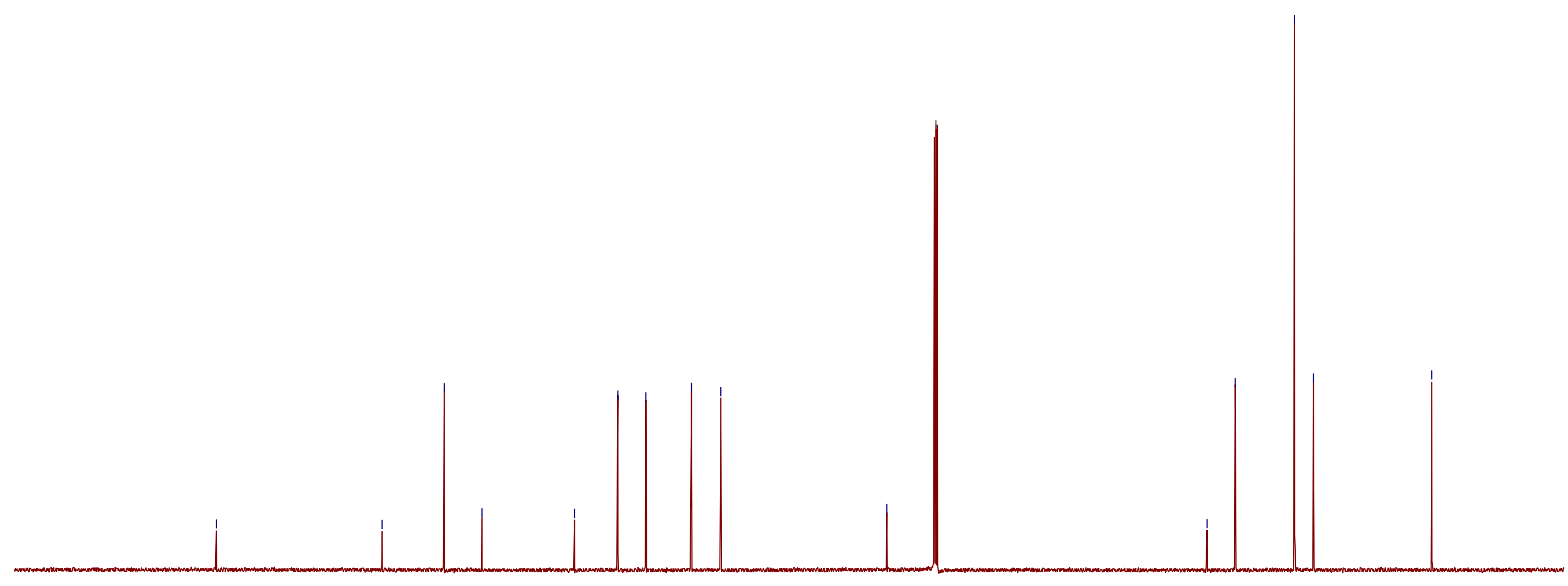




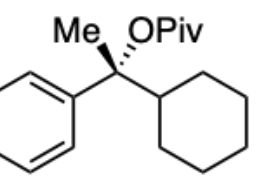

Compound 1i

${ }^{1} \mathrm{H}$ NMR $\left(600 \mathrm{MHz}, \mathrm{CDCl}_{3}\right)$

Whathen

10
10




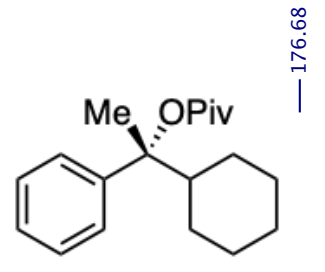

Compound 1i

${ }^{13} \mathrm{C}$ NMR $\left(151 \mathrm{MHz}, \mathrm{CDCl}_{3}\right)$ 


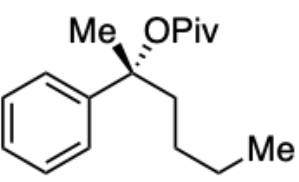

Compound $1 \mathrm{j}$

${ }^{1} \mathrm{H}$ NMR $\left(600 \mathrm{MHz}, \mathrm{CDCl}_{3}\right.$ )

'H NMR (6OO MHz, CDCl) 


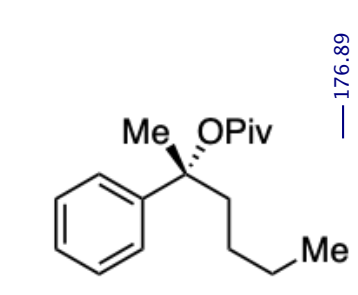

Compound $1 \mathrm{j}$

${ }^{13} \mathrm{C}$ NMR $\left(151 \mathrm{MHz}, \mathrm{CDCl}_{3}\right.$ )

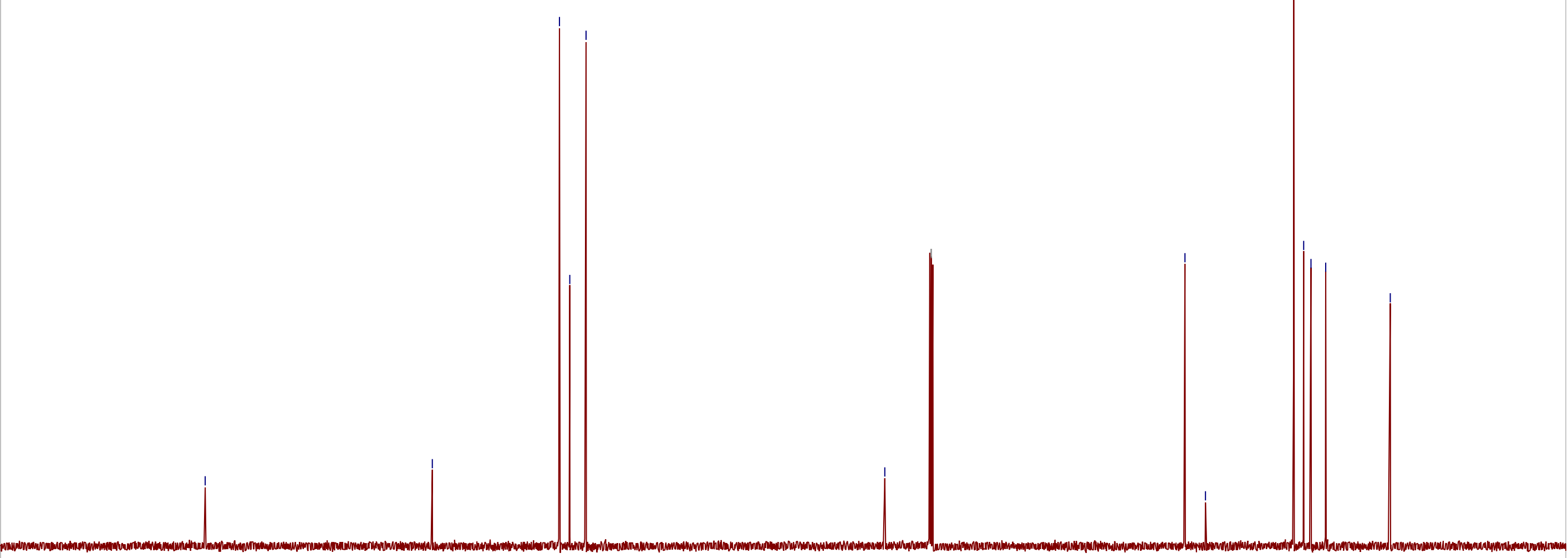




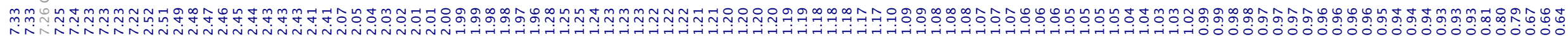

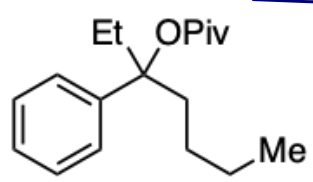

Compound 1k

${ }^{1} \mathrm{H}$ NMR $\left(600 \mathrm{MHz}, \mathrm{CDCl}_{3}\right)$

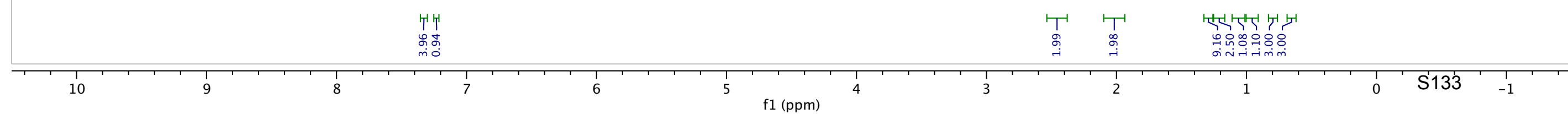




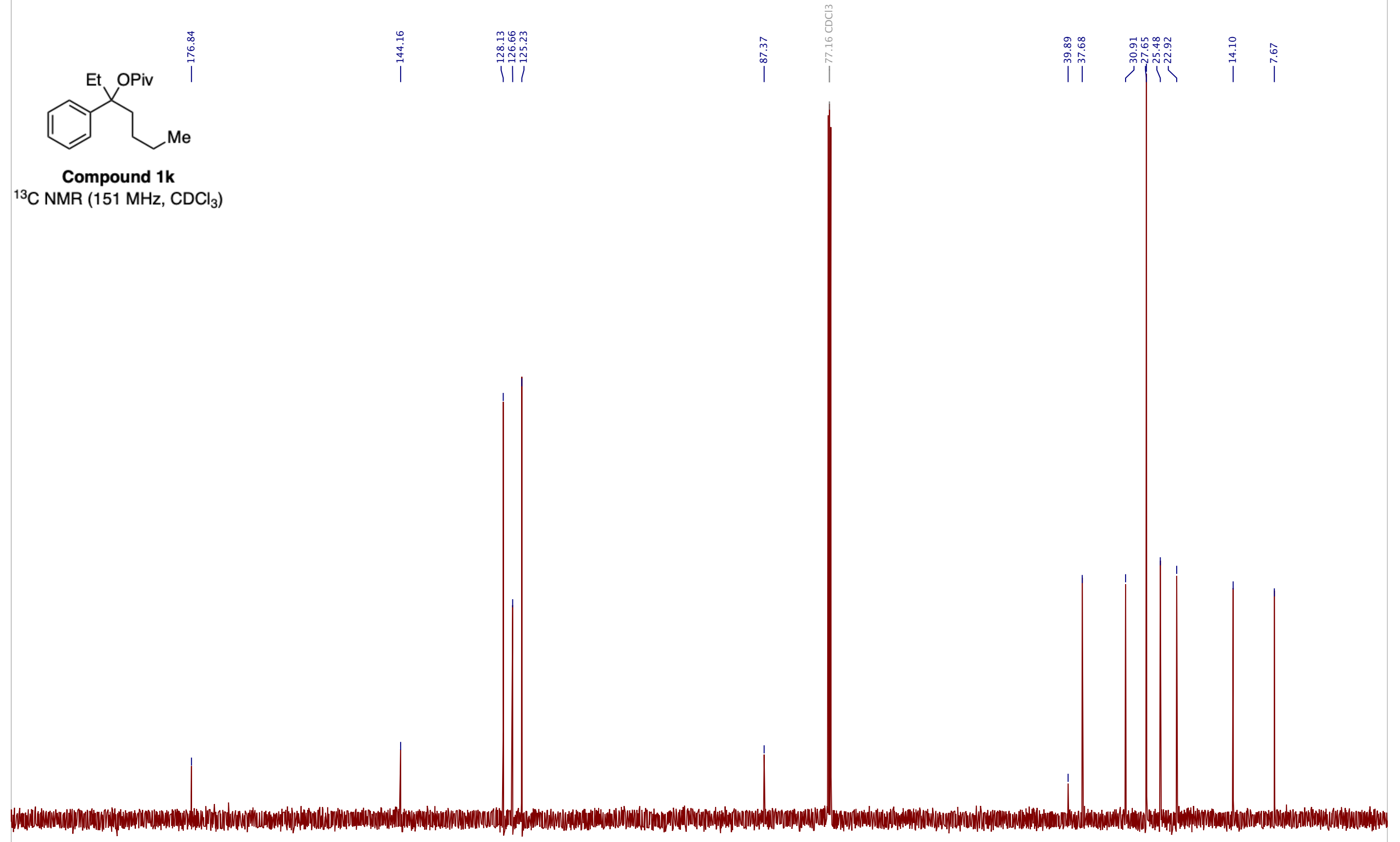


$\mathrm{Me}$ Et

$\mathrm{OH}$

\section{Compound $\mathrm{OH}-4$}

${ }^{1} \mathrm{H} \mathrm{NMR}\left(600 \mathrm{MHz}, \mathrm{CDCl}_{3}\right)$

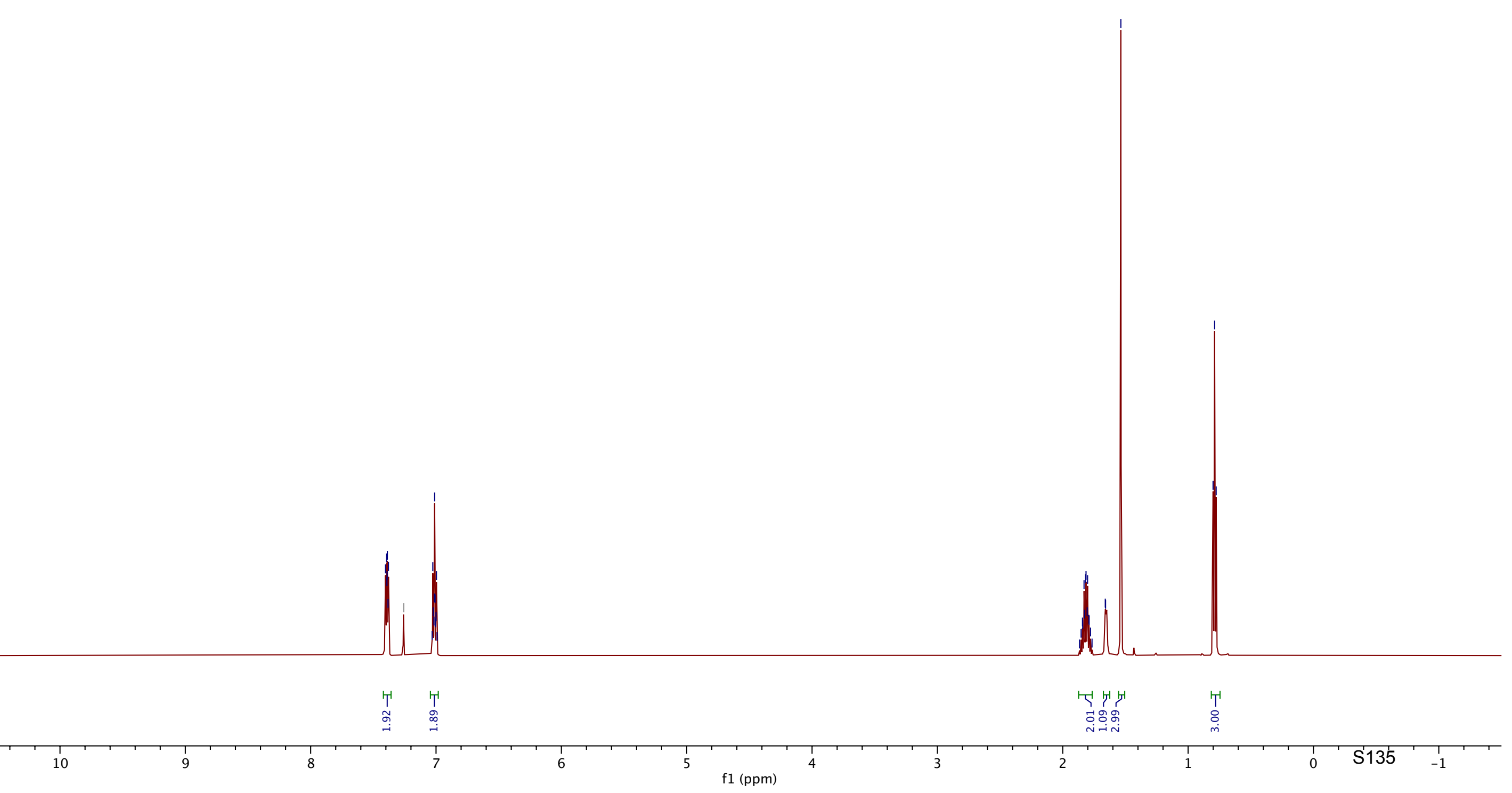


Compound $\mathrm{OH}-4$

${ }^{13} \mathrm{C}$ NMR (151 MHz, $\mathrm{CDCl}_{3}$ )

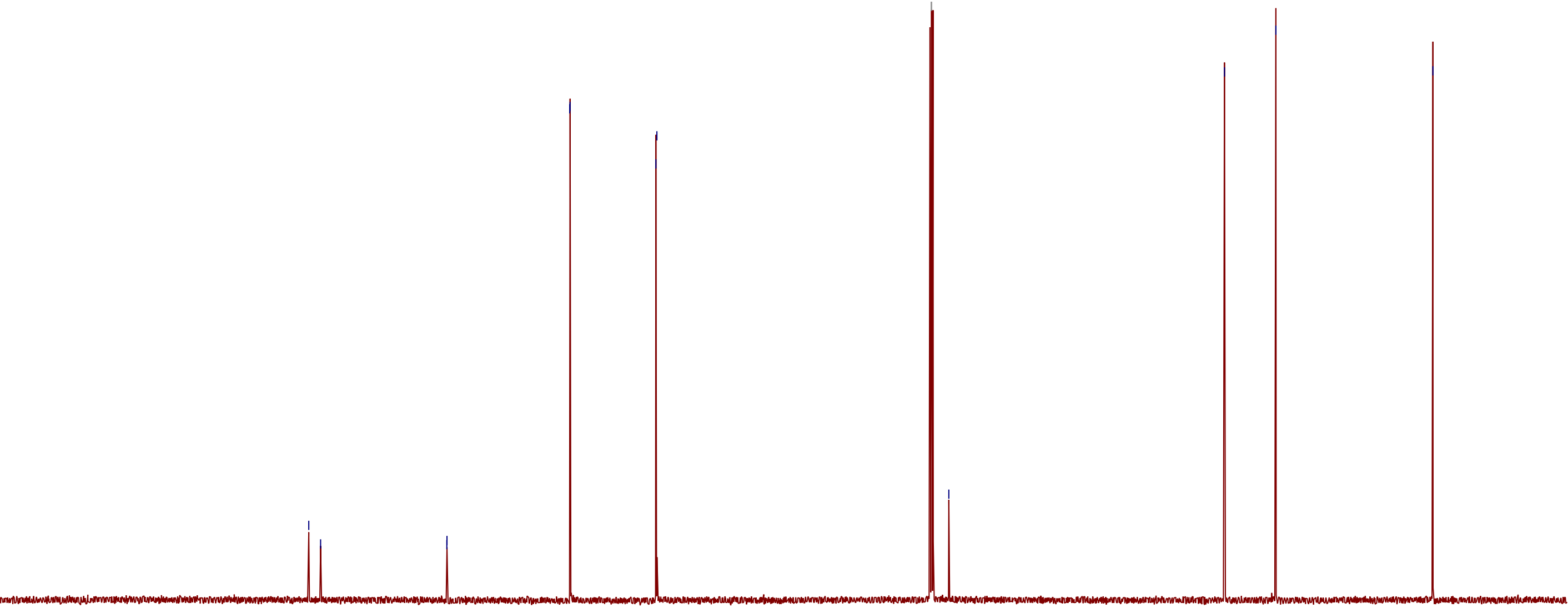




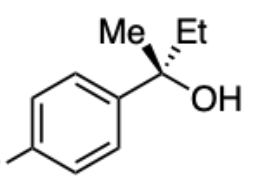

Compound OH-4

${ }^{19} \mathrm{~F}$ NMR $\left(565 \mathrm{MHz}, \mathrm{CDCl}_{3}\right.$ )

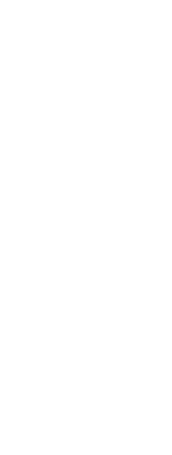




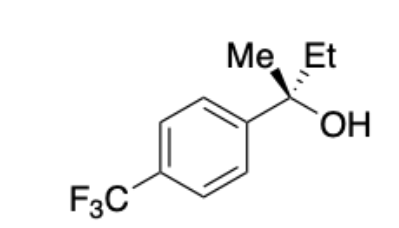

Compound OH-5

${ }^{1} \mathrm{H}$ NMR $\left(600 \mathrm{MHz}, \mathrm{CDCl}_{3}\right.$ )

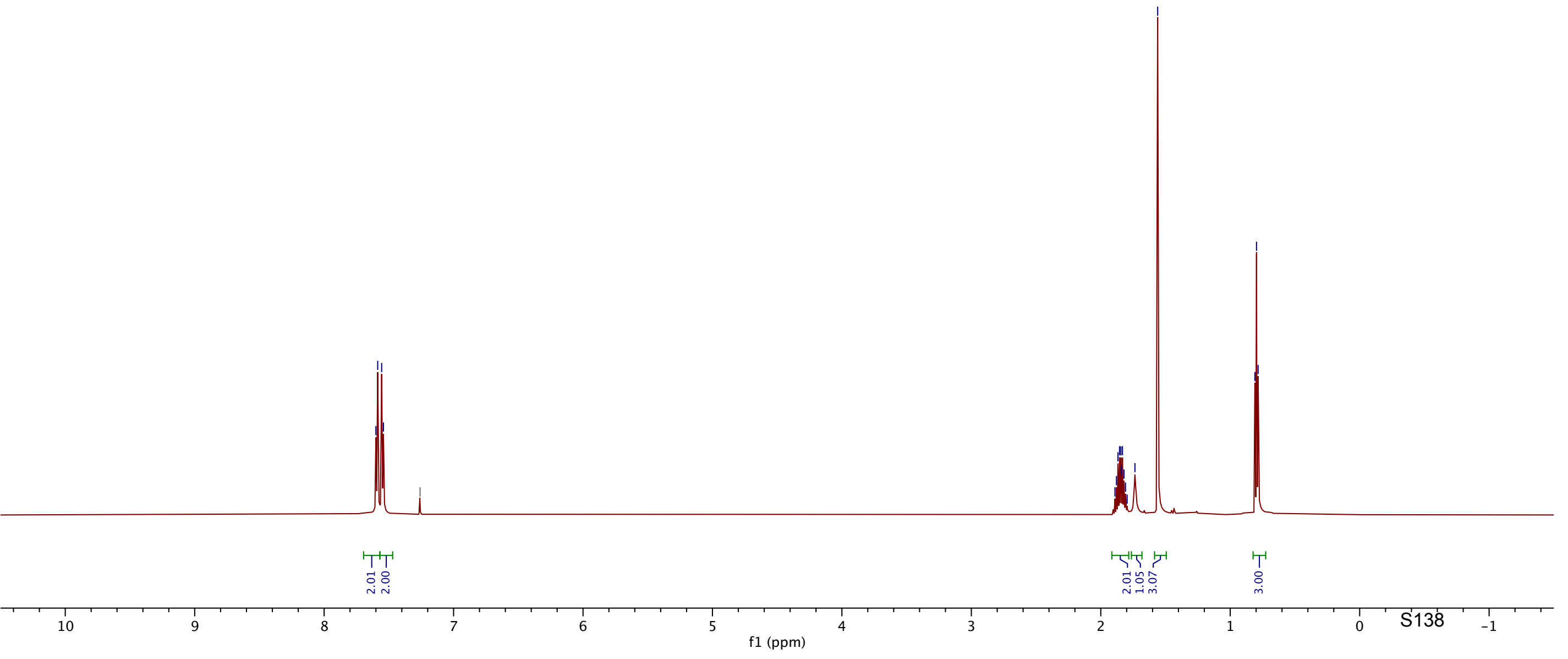




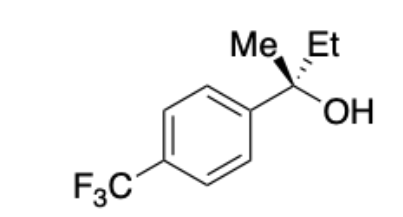

Compound $\mathrm{OH}-5$

${ }^{13} \mathrm{C}$ NMR $\left(151 \mathrm{MHz}, \mathrm{CDCl}_{3}\right)$

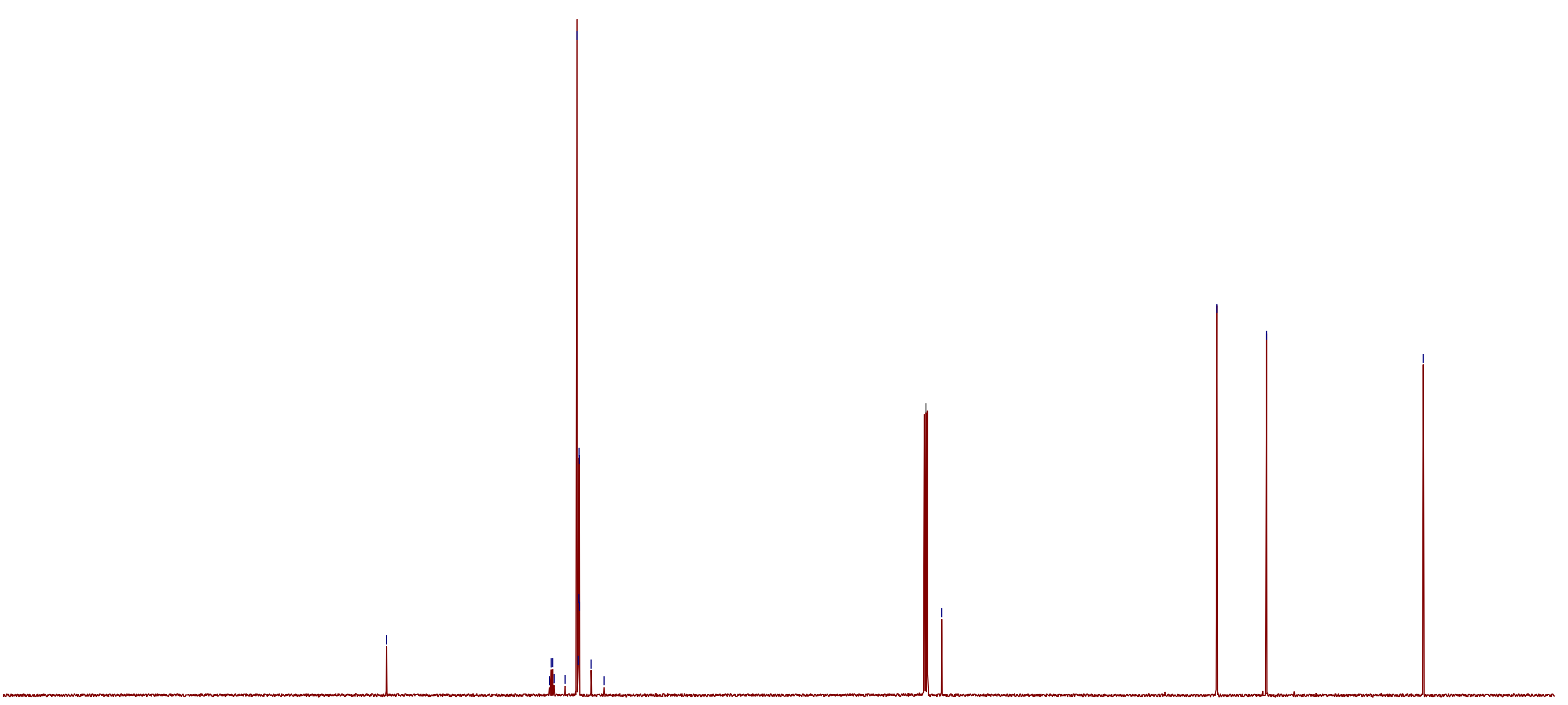

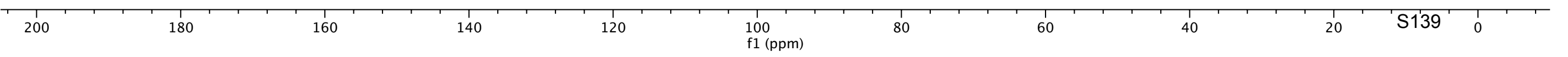




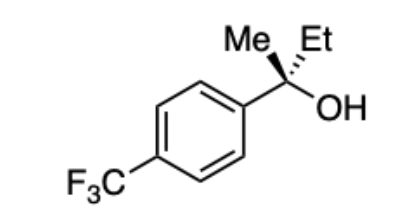

Compound $\mathrm{OH}-5$

${ }^{19} \mathrm{~F}$ NMR $\left(565 \mathrm{MHz}, \mathrm{CDCl}_{3}\right)$

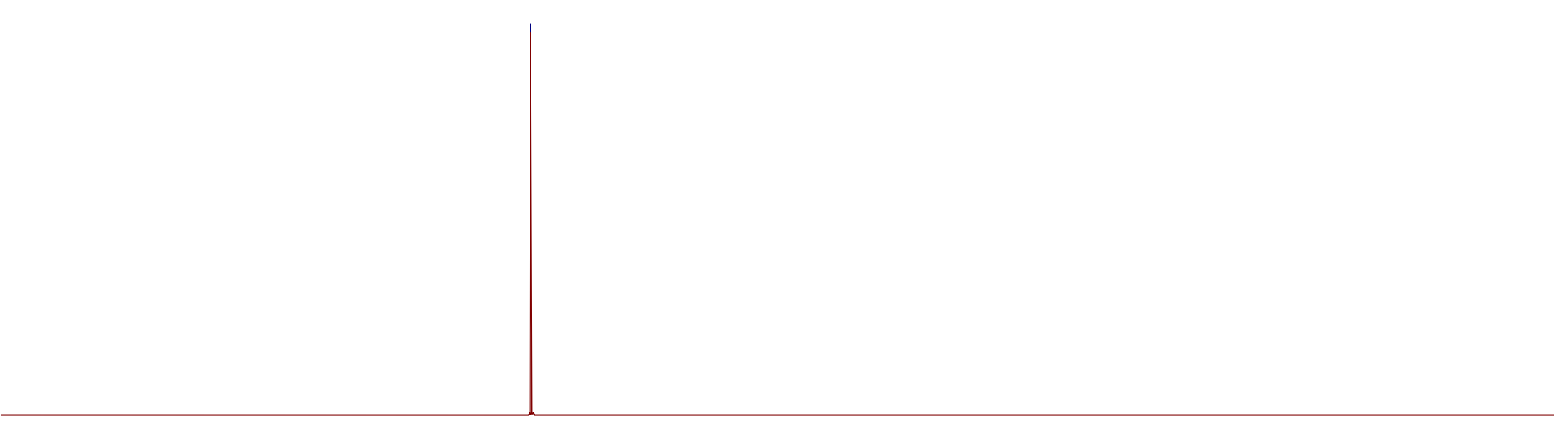




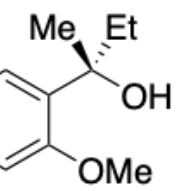

Compound $\mathrm{OH}-6$

${ }^{1} \mathrm{H}$ NMR $\left(600 \mathrm{MHz}, \mathrm{CDCl}_{3}\right.$ )

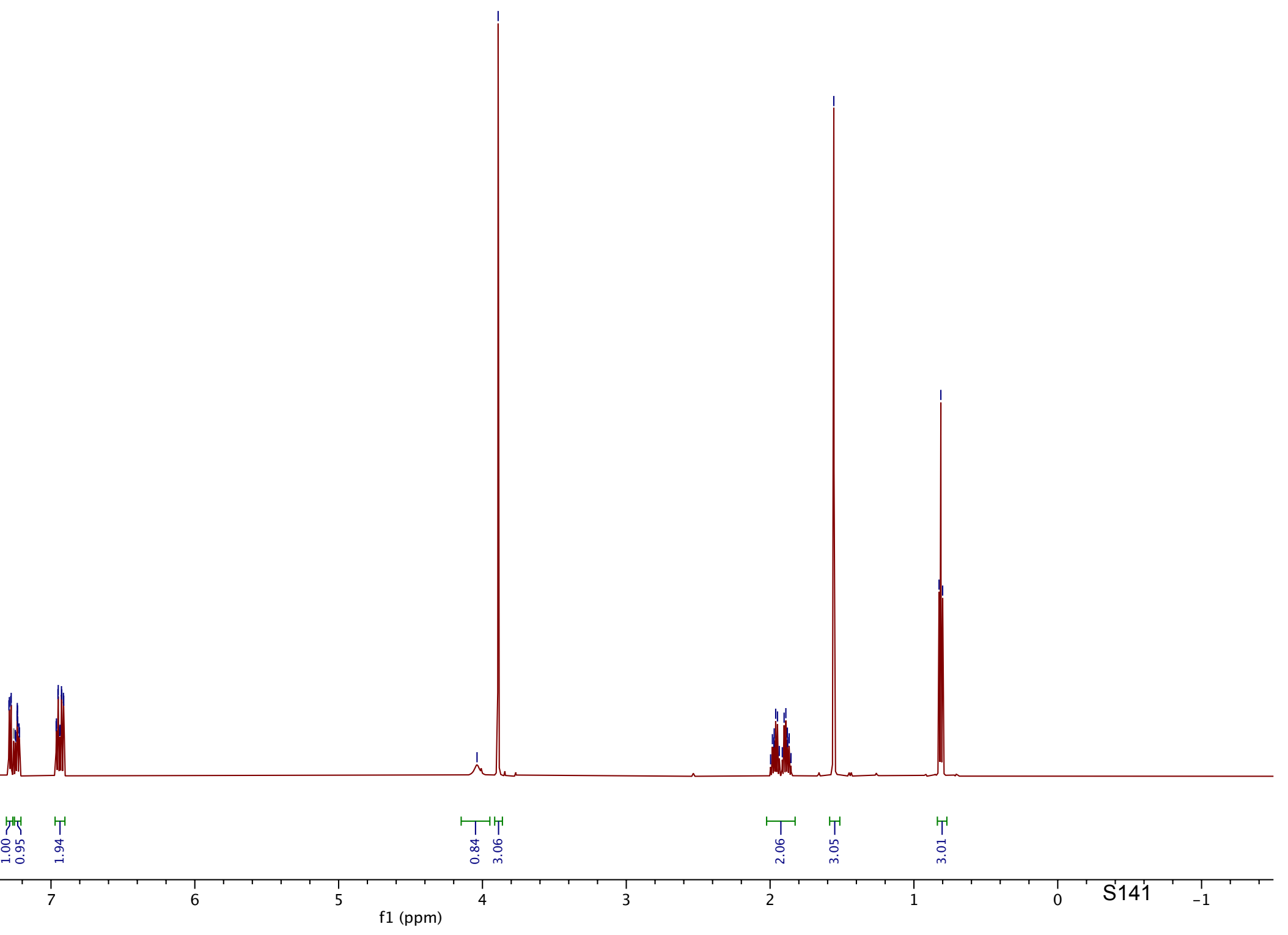




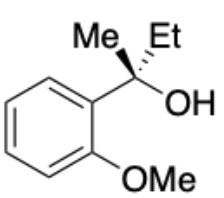

Compound $\mathrm{OH}-6$

${ }^{13} \mathrm{C}$ NMR $\left(151 \mathrm{MHz}, \mathrm{CDCl}_{3}\right)$ 


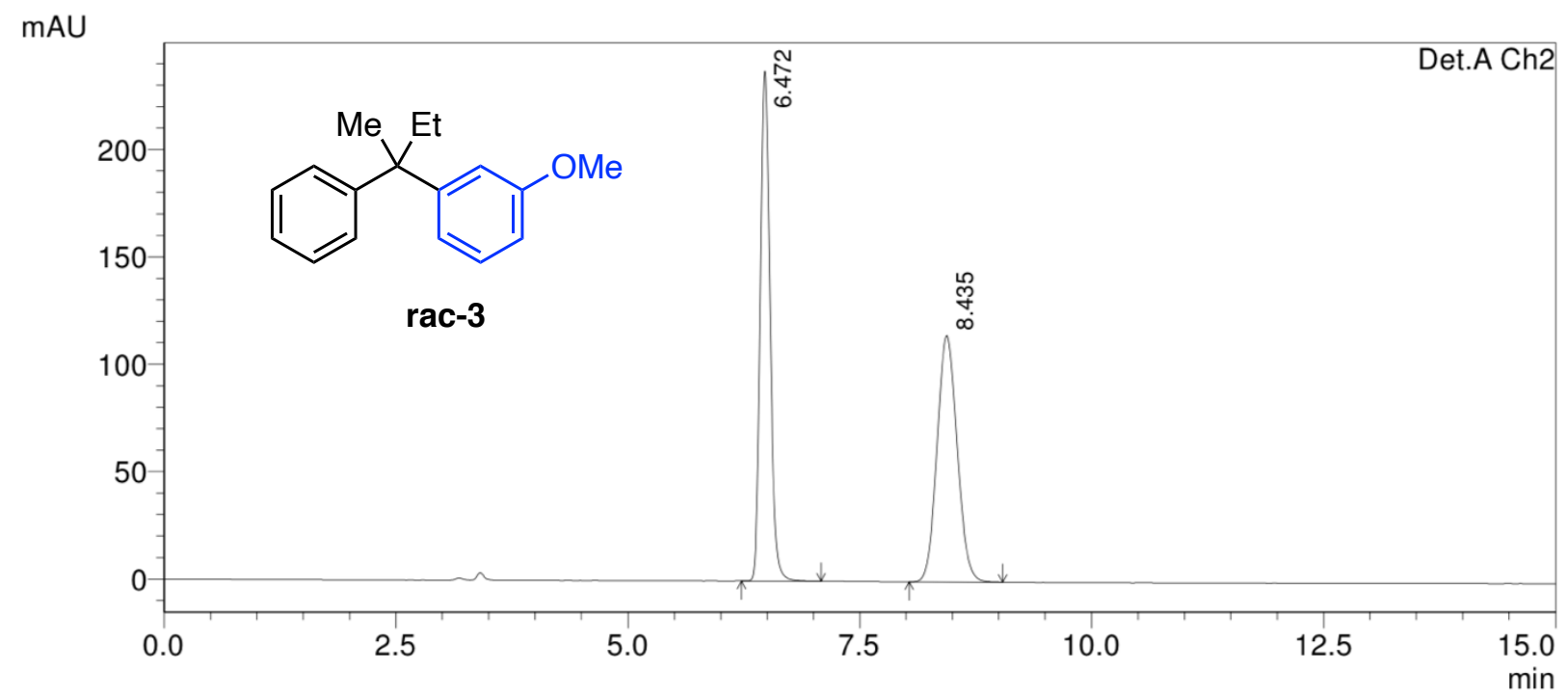

Detector A Ch2 220nm

\begin{tabular}{|r|r|r|r|r|r|}
\hline \multicolumn{1}{|c|}{ Peak\# } & Ret. Time & \multicolumn{1}{|c|}{ Area } & Height & Area \% & Height \% \\
\hline 1 & 6.472 & 1731374 & 237313 & 50.011 & 67.391 \\
\hline 2 & 8.435 & 1730607 & 114828 & 49.989 & 32.609 \\
\hline Total & & 3461980 & 352141 & 100.000 & 100.000 \\
\hline
\end{tabular}

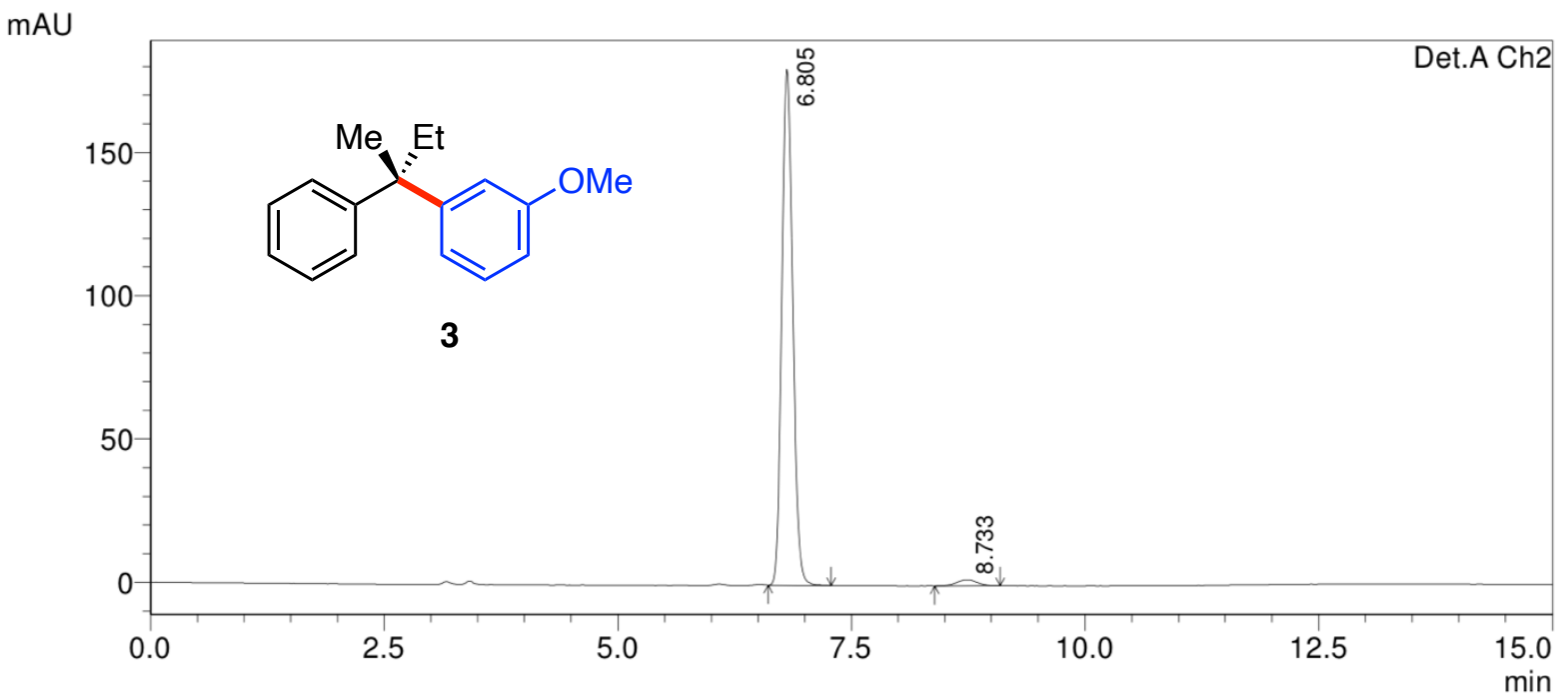

Detector A Ch2 220nm

\begin{tabular}{|r|r|r|r|r|r|}
\hline \multicolumn{1}{|c|}{ Peak\# } & \multicolumn{1}{|c|}{ Ret. Time } & \multicolumn{1}{c|}{ Area } & \multicolumn{1}{c|}{ Height } & Area \% & \multicolumn{1}{|c|}{ Height \% } \\
\hline 1 & 6.805 & 1459500 & 179928 & 97.868 & 98.852 \\
\hline 2 & 8.733 & 31794 & 2090 & 2.132 & 1.148 \\
\hline Total & & 1491295 & 182018 & 100.000 & 100.000 \\
\hline
\end{tabular}




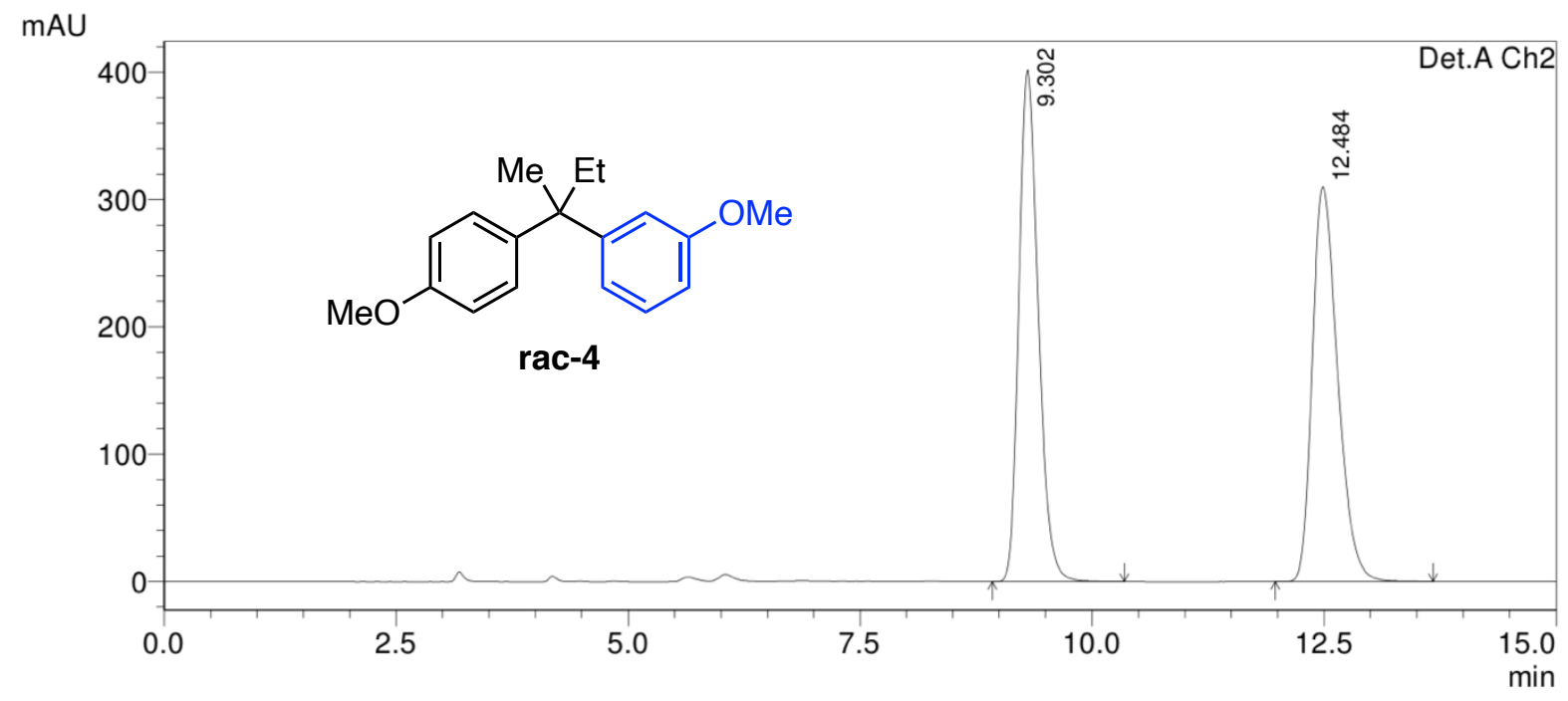

Detector A Ch2 220nm

\begin{tabular}{|r|r|r|r|r|r|}
\hline \multicolumn{1}{|c|}{ Peak\# } & Ret. Time & \multicolumn{1}{c|}{ Area } & Height & \multicolumn{1}{|c|}{ Area \% } & \multicolumn{1}{c|}{ Height \% } \\
\hline 1 & 9.302 & 5858073 & 401975 & 49.830 & 56.458 \\
\hline 2 & 12.484 & 5898152 & 310012 & 50.170 & 43.542 \\
\hline Total & & 11756226 & 711987 & 100.000 & 100.000 \\
\hline
\end{tabular}

mAU

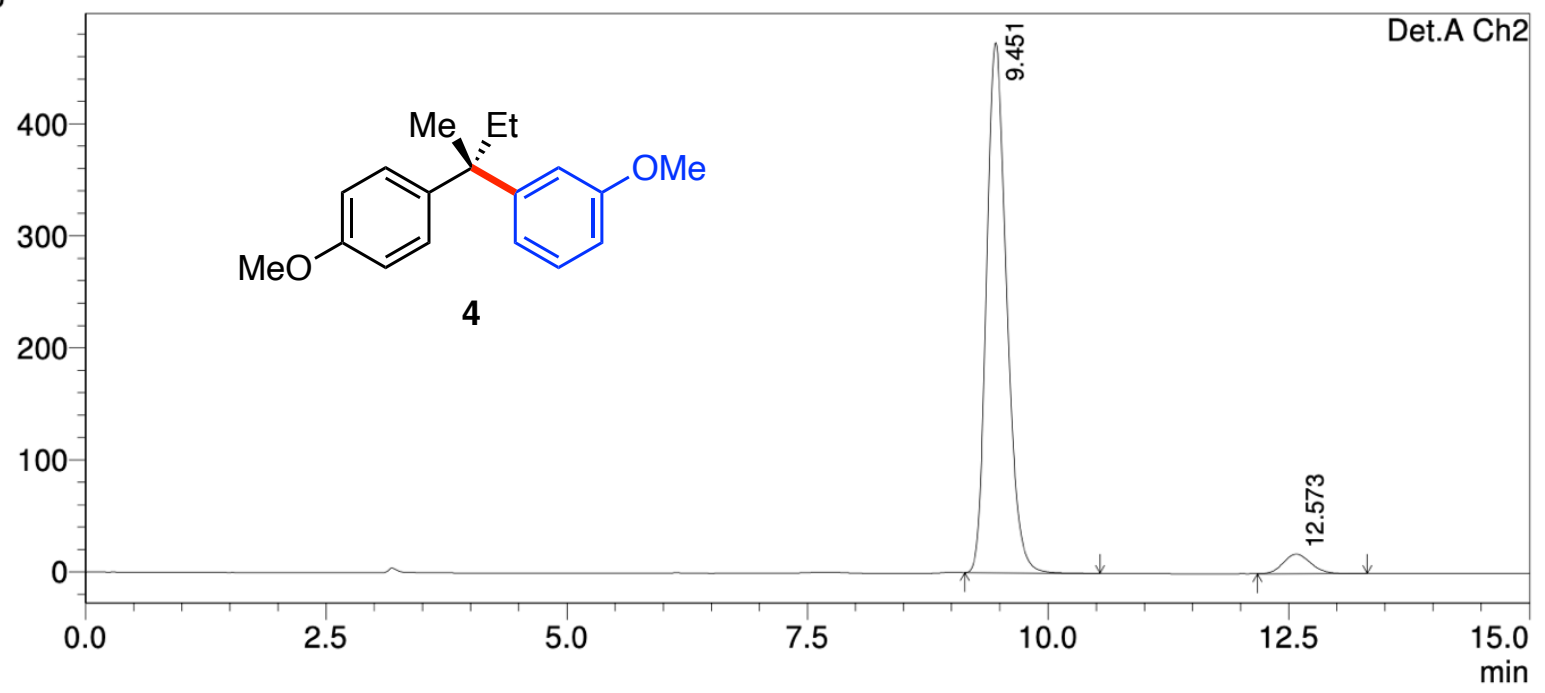

Detector A Ch2 220nm

\begin{tabular}{|r|r|r|r|r|r|}
\hline \multicolumn{1}{|c|}{ Peak\# } & Ret. Time & \multicolumn{1}{|c|}{ Area } & Height & Area \% & \multicolumn{1}{c|}{ Height \% } \\
\hline 1 & 9.451 & 6883291 & 472837 & 95.196 & 96.448 \\
\hline 2 & 12.573 & 347353 & 17414 & 4.804 & 3.552 \\
\hline Total & & 7230643 & 490251 & 100.000 & 100.000 \\
\hline
\end{tabular}




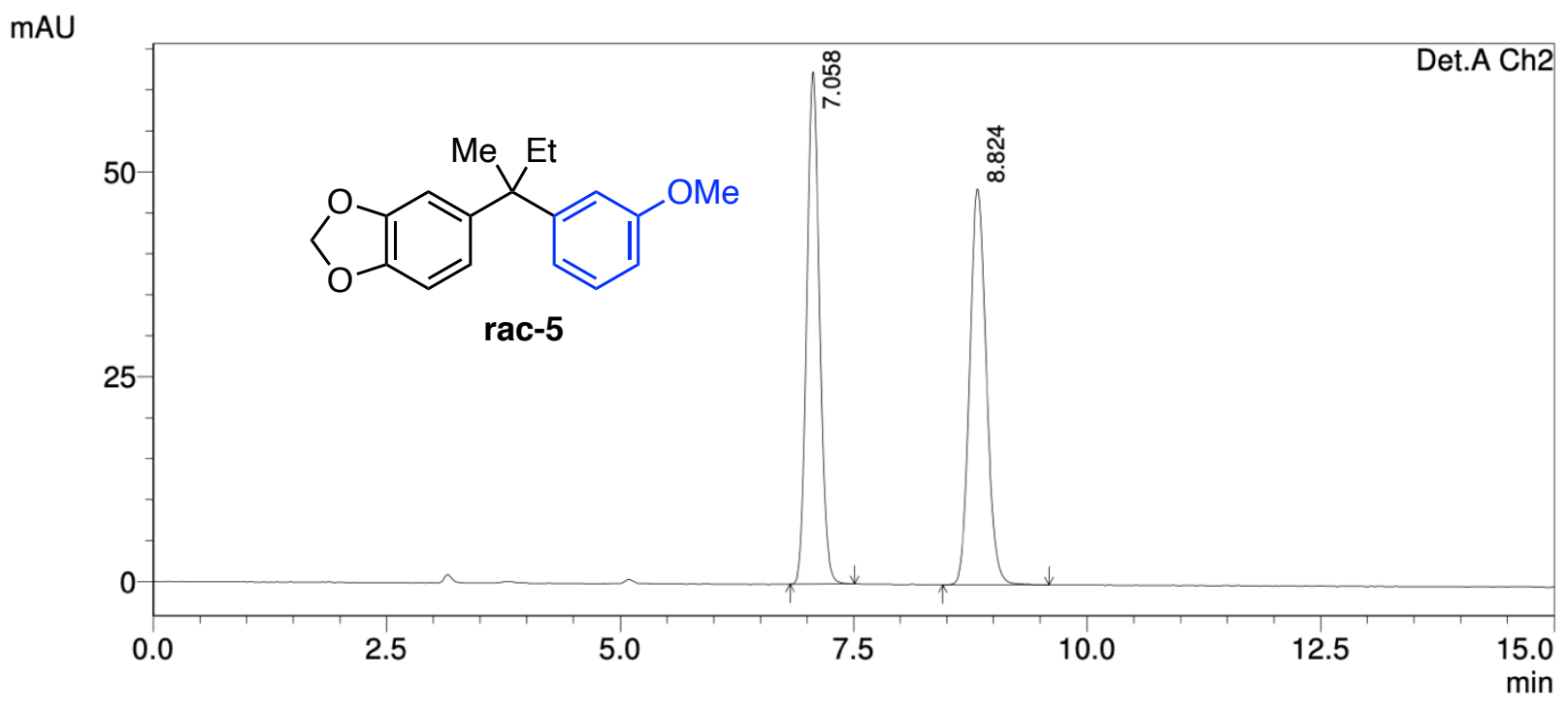

Detector A Ch2 220nm

\begin{tabular}{|r|r|r|r|r|r|}
\hline \multicolumn{1}{|c|}{ Peak\# } & Ret. Time & \multicolumn{1}{c|}{ Area } & Height & Area \% & \multicolumn{1}{|c|}{ Height \% } \\
\hline 1 & 7.058 & 597234 & 62439 & 49.883 & 56.377 \\
\hline 2 & 8.824 & 600034 & 48315 & 50.117 & 43.623 \\
\hline Total & & 1197268 & 110754 & 100.000 & 100.000 \\
\hline
\end{tabular}

mAU

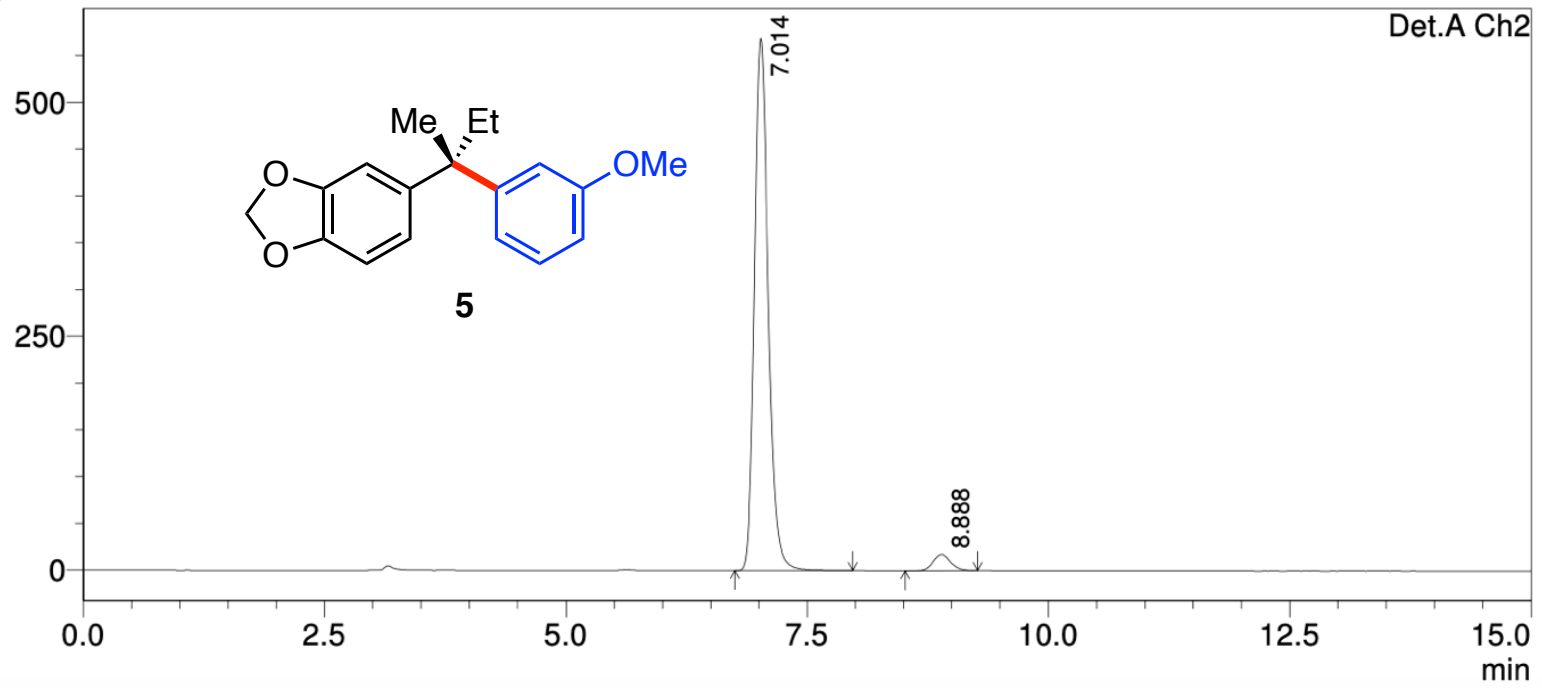

Detector A Ch2 220nm

\begin{tabular}{|r|r|r|r|r|r|}
\hline \multicolumn{1}{|c|}{ Peak\# } & Ret. Time & \multicolumn{1}{|c|}{ Area } & Height & Area \% & Height \% \\
\hline 1 & 7.014 & 5653872 & 568644 & 96.331 & 97.082 \\
\hline 2 & 8.888 & 215365 & 17089 & 3.669 & 2.918 \\
\hline Total & & 5869236 & 585733 & 100.000 & 100.000 \\
\hline
\end{tabular}




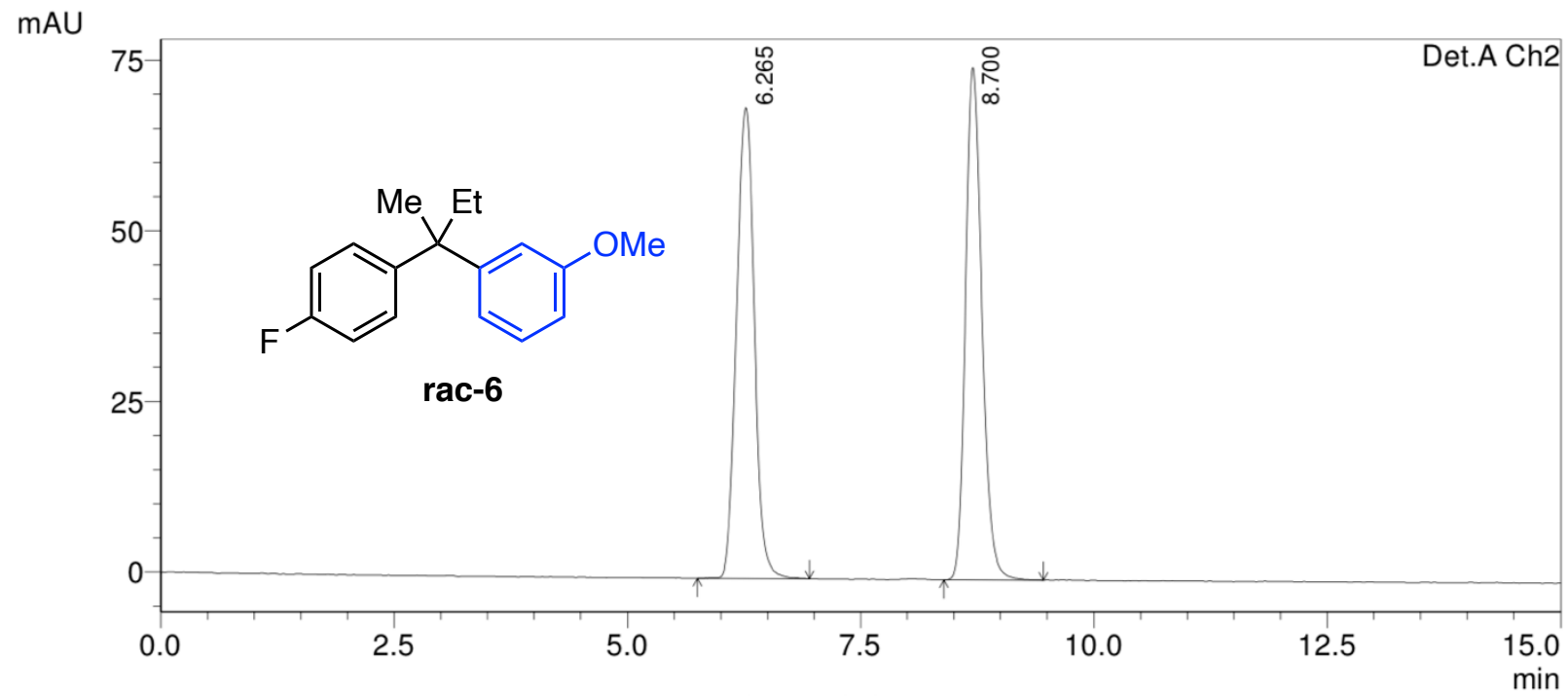

Detector A Ch2 280nm

\begin{tabular}{|r|r|r|r|r|r|}
\hline \multicolumn{1}{|c|}{ Peak\# } & Ret. Time & \multicolumn{1}{c|}{ Area } & Height & Area \% & \multicolumn{1}{|c|}{ Height \% } \\
\hline 1 & 6.265 & 922539 & 68993 & 50.074 & 47.902 \\
\hline 2 & 8.700 & 919822 & 75036 & 49.926 & 52.098 \\
\hline Total & & 1842361 & 144029 & 100.000 & 100.000 \\
\hline
\end{tabular}

mAU

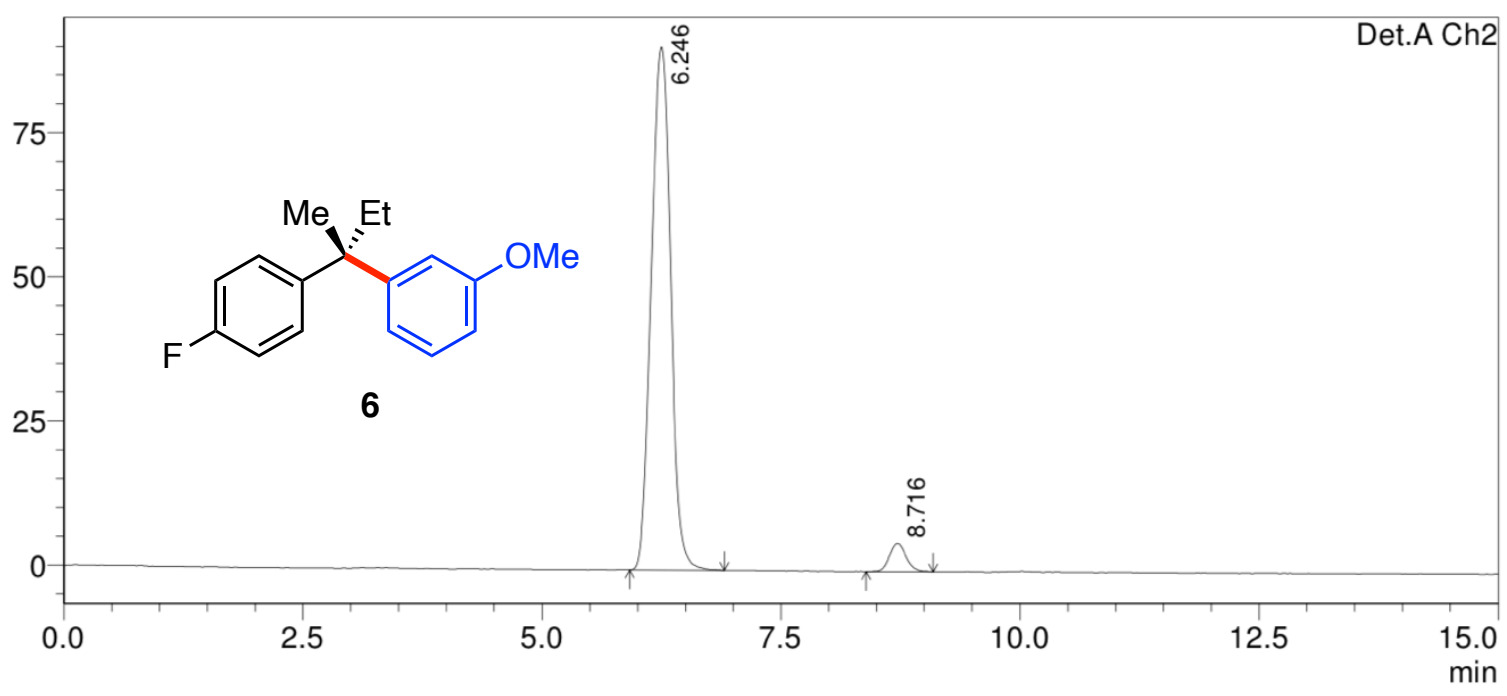

Detector A Ch2 280nm

\begin{tabular}{|r|r|r|r|r|r|}
\hline \multicolumn{1}{|c|}{ Peak\# } & Ret. Time & \multicolumn{1}{|c|}{ Area } & Height & Area \% & \multicolumn{1}{c|}{ Height \% } \\
\hline 1 & 6.246 & 1284082 & 90792 & 95.502 & 94.835 \\
\hline 2 & 8.716 & 60478 & 4945 & 4.498 & 5.165 \\
\hline Total & & 1344559 & 95736 & 100.000 & 100.000 \\
\hline
\end{tabular}




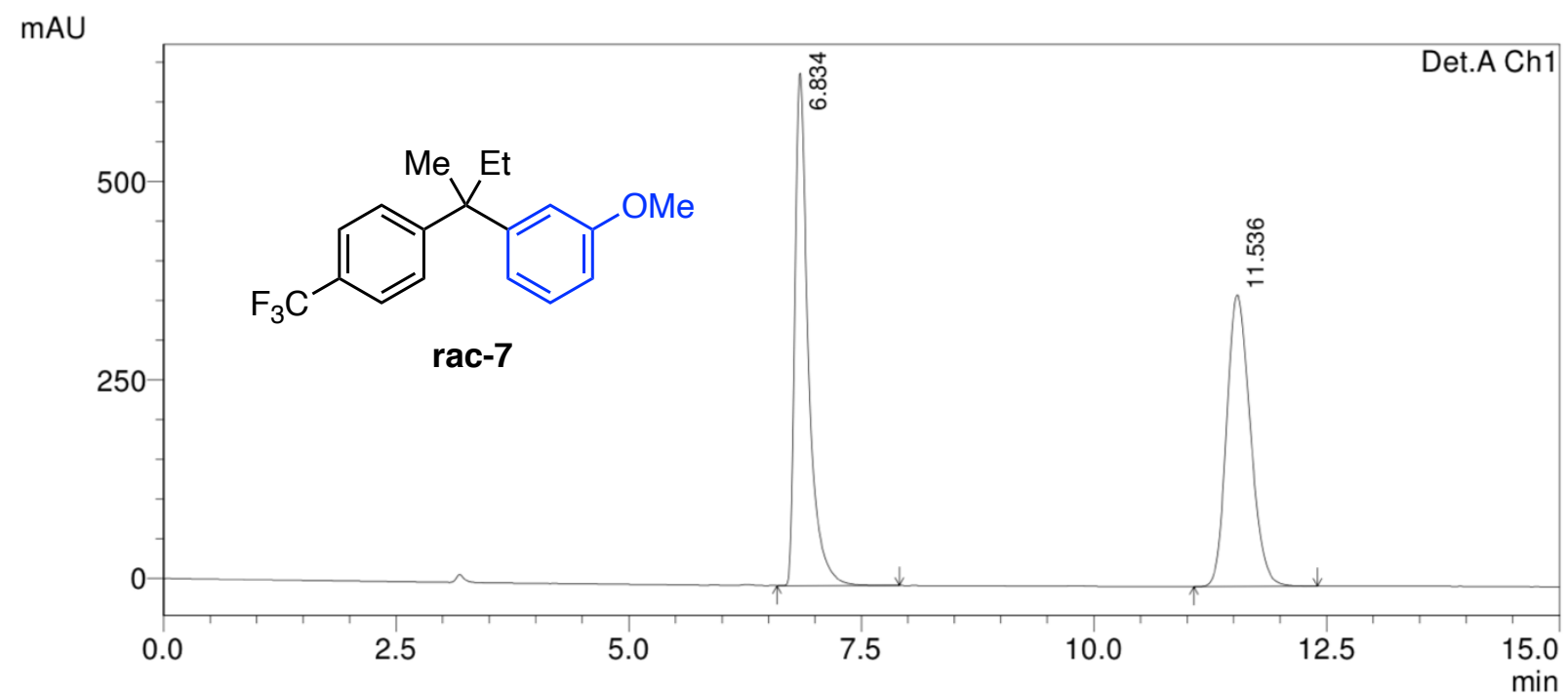

Detector A Ch1 210nm

\begin{tabular}{|r|r|r|r|r|r|}
\hline \multicolumn{1}{|c|}{ Peak\# } & Ret. Time & \multicolumn{1}{c|}{ Area } & Height & \multicolumn{1}{c|}{ Area \% } & Height \% \\
\hline 1 & 6.834 & 6455550 & 645489 & 49.788 & 63.733 \\
\hline 2 & 11.536 & 6510607 & 367316 & 50.212 & 36.267 \\
\hline Total & & 12966156 & 1012805 & 100.000 & 100.000 \\
\hline
\end{tabular}

mAU

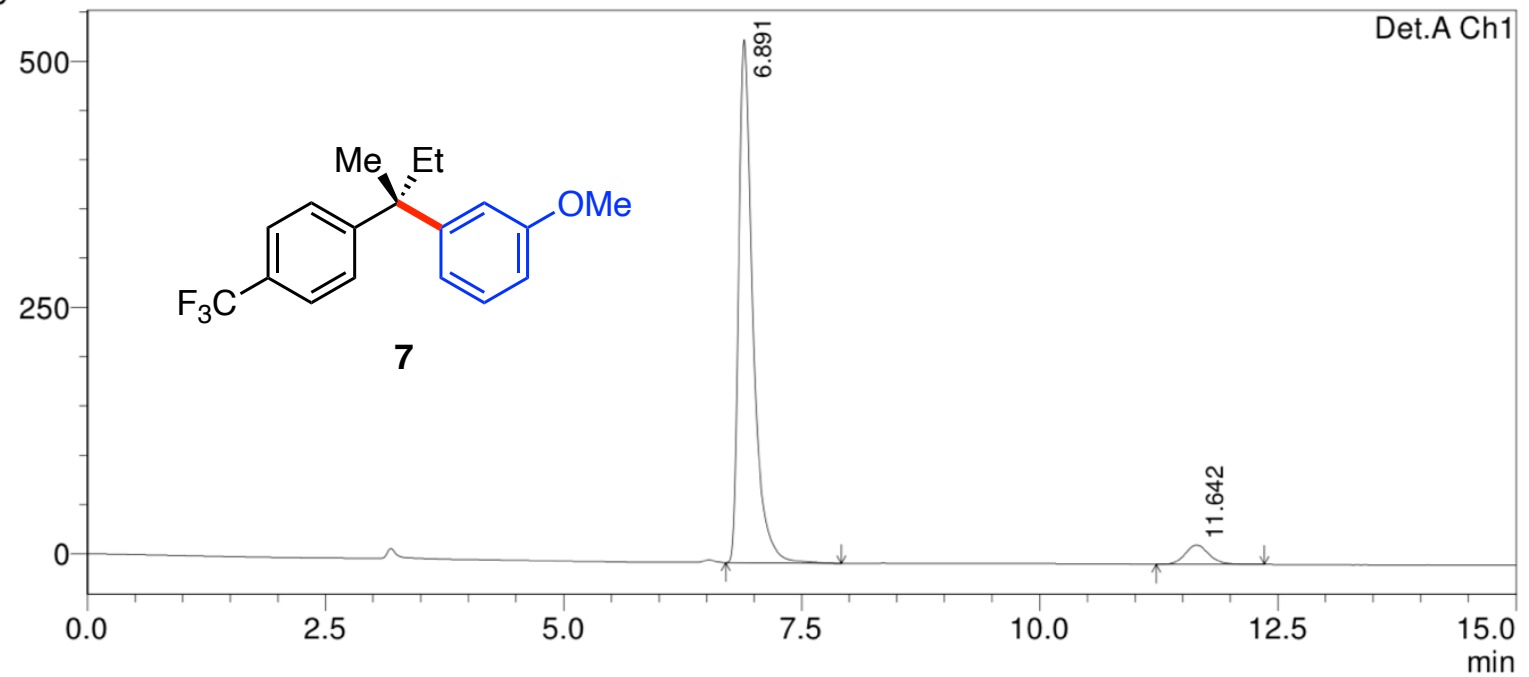

Detector A Ch1 210nm

\begin{tabular}{|r|r|r|r|r|r|}
\hline \multicolumn{1}{|c|}{ Peak\# } & Ret. Time & \multicolumn{1}{|c|}{ Area } & Height & Area \% & Height \% \\
\hline 1 & 6.891 & 5531668 & 531058 & 94.156 & 96.461 \\
\hline 2 & 11.642 & 343352 & 19486 & 5.844 & 3.539 \\
\hline Total & & 5875020 & 550544 & 100.000 & 100.000 \\
\hline
\end{tabular}




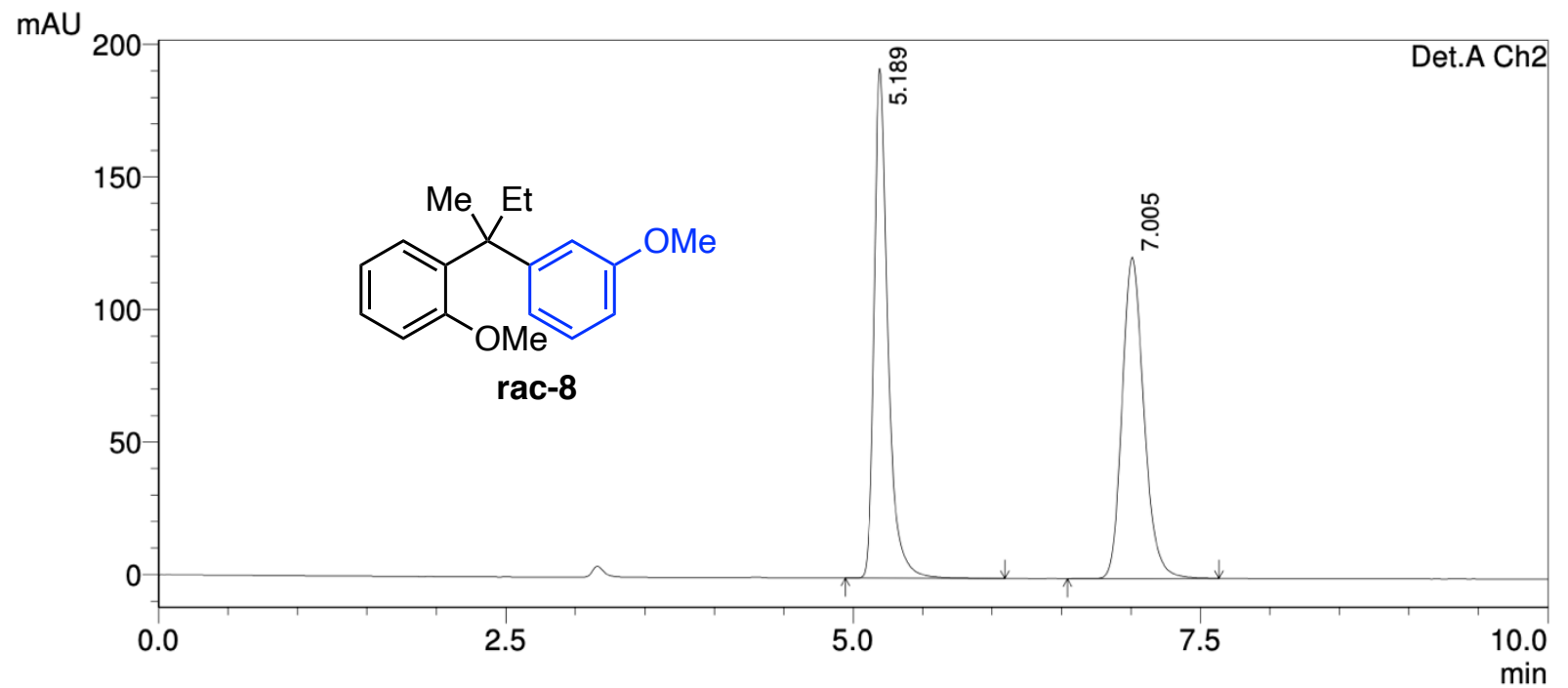

Detector A Ch2 220nm

\begin{tabular}{|r|r|r|r|r|r|}
\hline \multicolumn{1}{|c|}{ Peak\# } & Ret. Time & \multicolumn{1}{|c|}{ Area } & Height & Area \% & Height \% \\
\hline 1 & 5.189 & 1334380 & 192136 & 50.179 & 61.321 \\
\hline 2 & 7.005 & 1324871 & 121192 & 49.821 & 38.679 \\
\hline Total & & 2659251 & 313329 & 100.000 & 100.000 \\
\hline
\end{tabular}

mAU

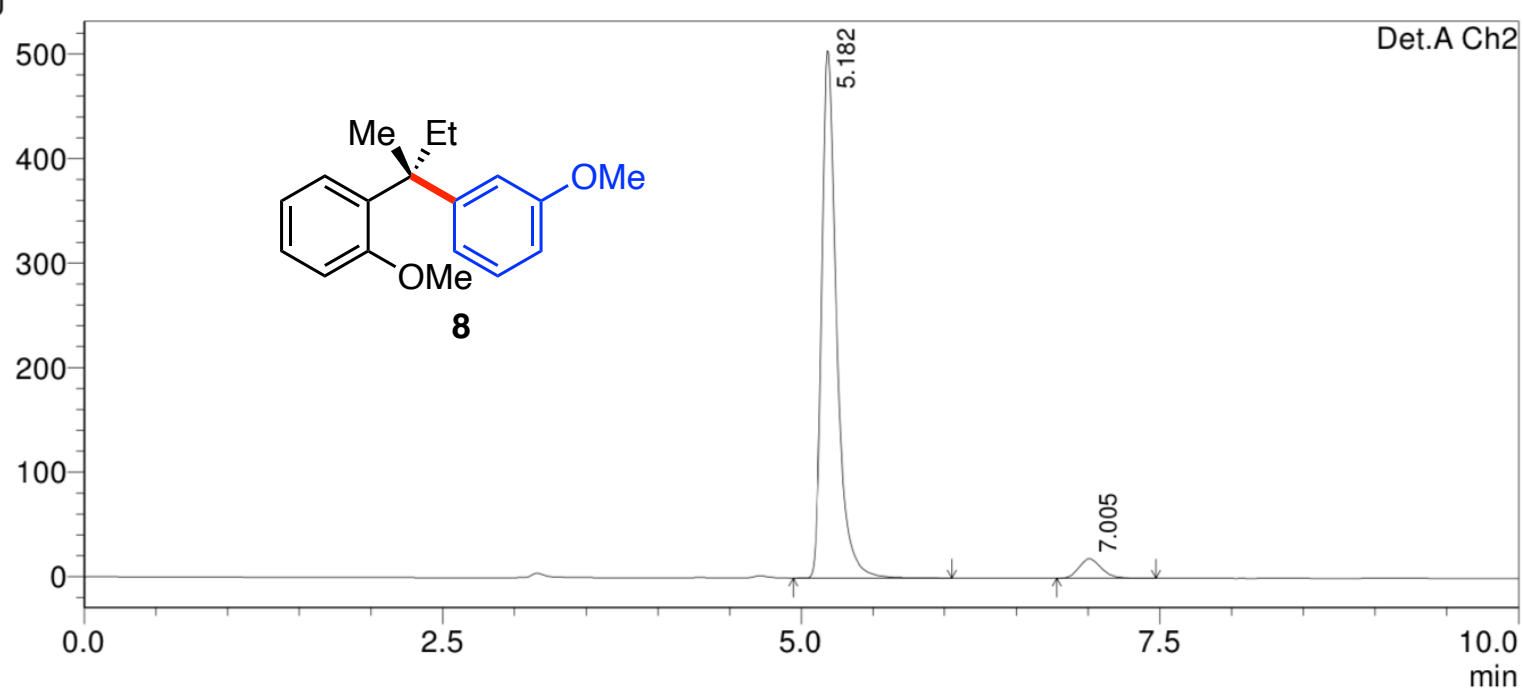

Detector A Ch2 220nm

\begin{tabular}{|r|r|r|r|r|r|}
\hline \multicolumn{1}{|c|}{ Peak\# } & Ret. Time & \multicolumn{1}{|c|}{ Area } & Height & Area \% & \multicolumn{1}{c|}{ Height \% } \\
\hline 1 & 5.182 & 3670318 & 504438 & 94.833 & 96.476 \\
\hline 2 & 7.005 & 199970 & 18426 & 5.167 & 3.524 \\
\hline Total & & 3870288 & 522864 & 100.000 & 100.000 \\
\hline
\end{tabular}




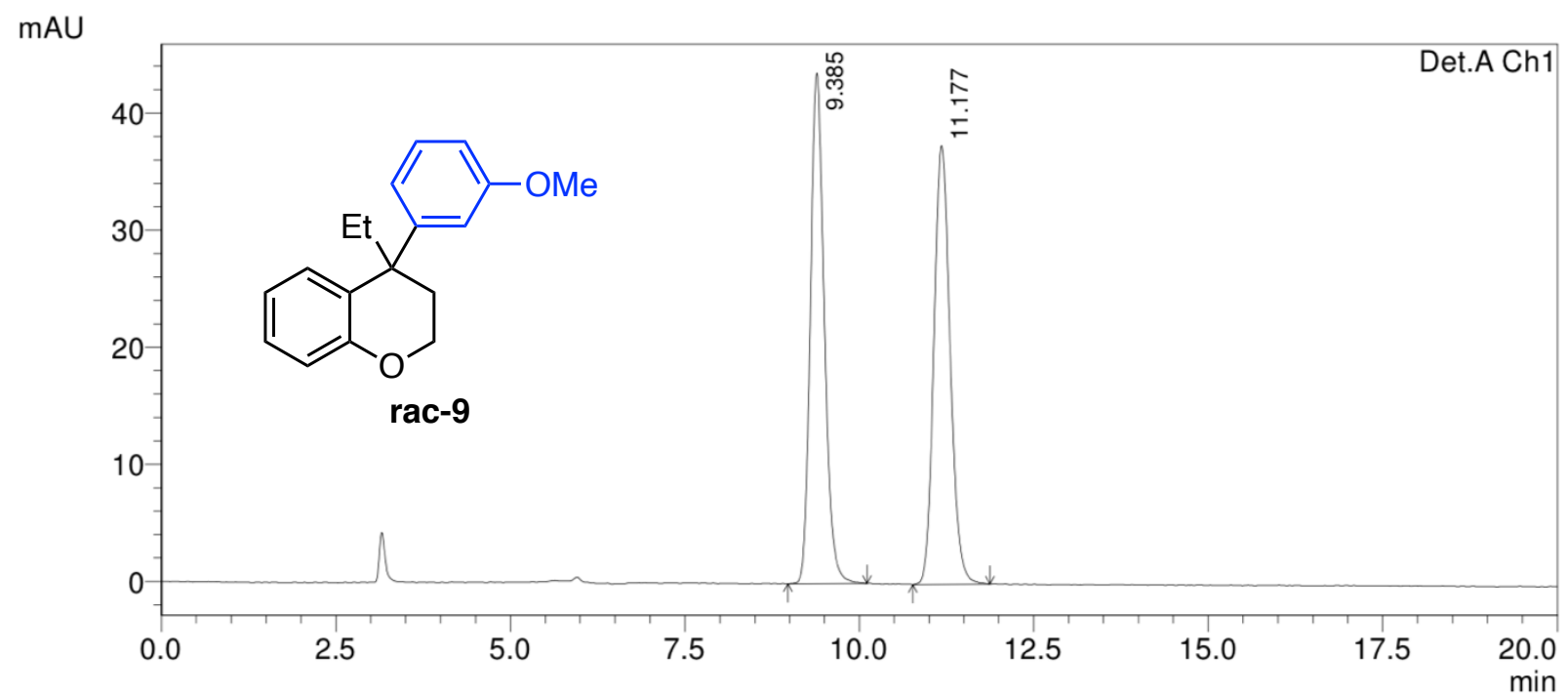

Detector A Ch1 220nm

\begin{tabular}{|r|r|r|r|r|r|}
\hline \multicolumn{1}{|c|}{ Peak\# } & Ret. Time & \multicolumn{1}{|c|}{ Area } & Height & Area \% & Height \% \\
\hline 1 & 9.385 & 607272 & 43625 & 49.947 & 53.786 \\
\hline 2 & 11.177 & 608560 & 37483 & 50.053 & 46.214 \\
\hline Total & & 1215833 & 81108 & 100.000 & 100.000 \\
\hline
\end{tabular}

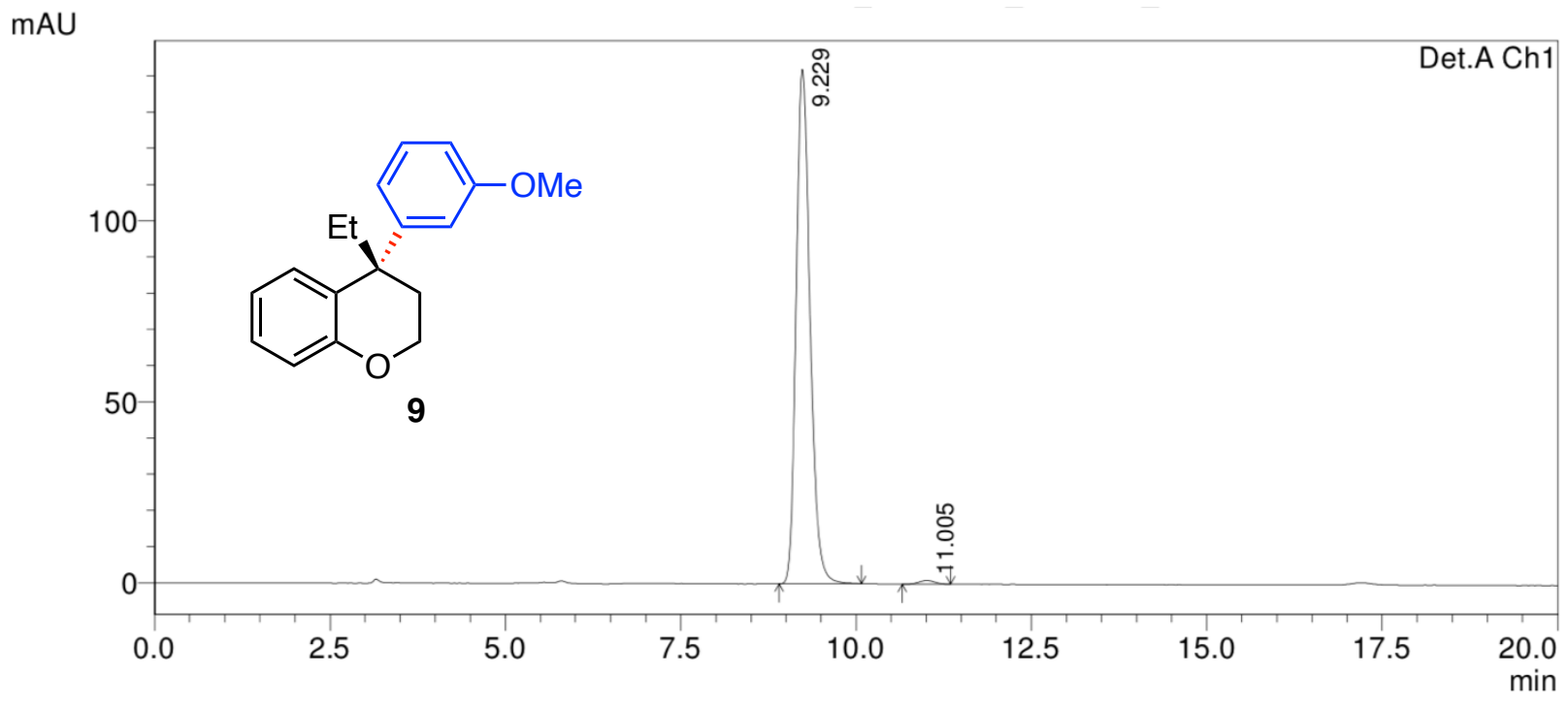

Detector A Ch1 220nm

\begin{tabular}{|r|r|r|r|r|r|}
\hline \multicolumn{1}{|c|}{ Peak\# } & \multicolumn{1}{|c|}{ Ret. Time } & \multicolumn{1}{|c|}{ Area } & Height & Area \% & Height \% \\
\hline 1 & 9.229 & 1951130 & 142036 & 99.173 & 99.282 \\
\hline 2 & 11.005 & 16271 & 1027 & 0.827 & 0.718 \\
\hline Total & & 1967402 & 143063 & 100.000 & 100.000 \\
\hline
\end{tabular}




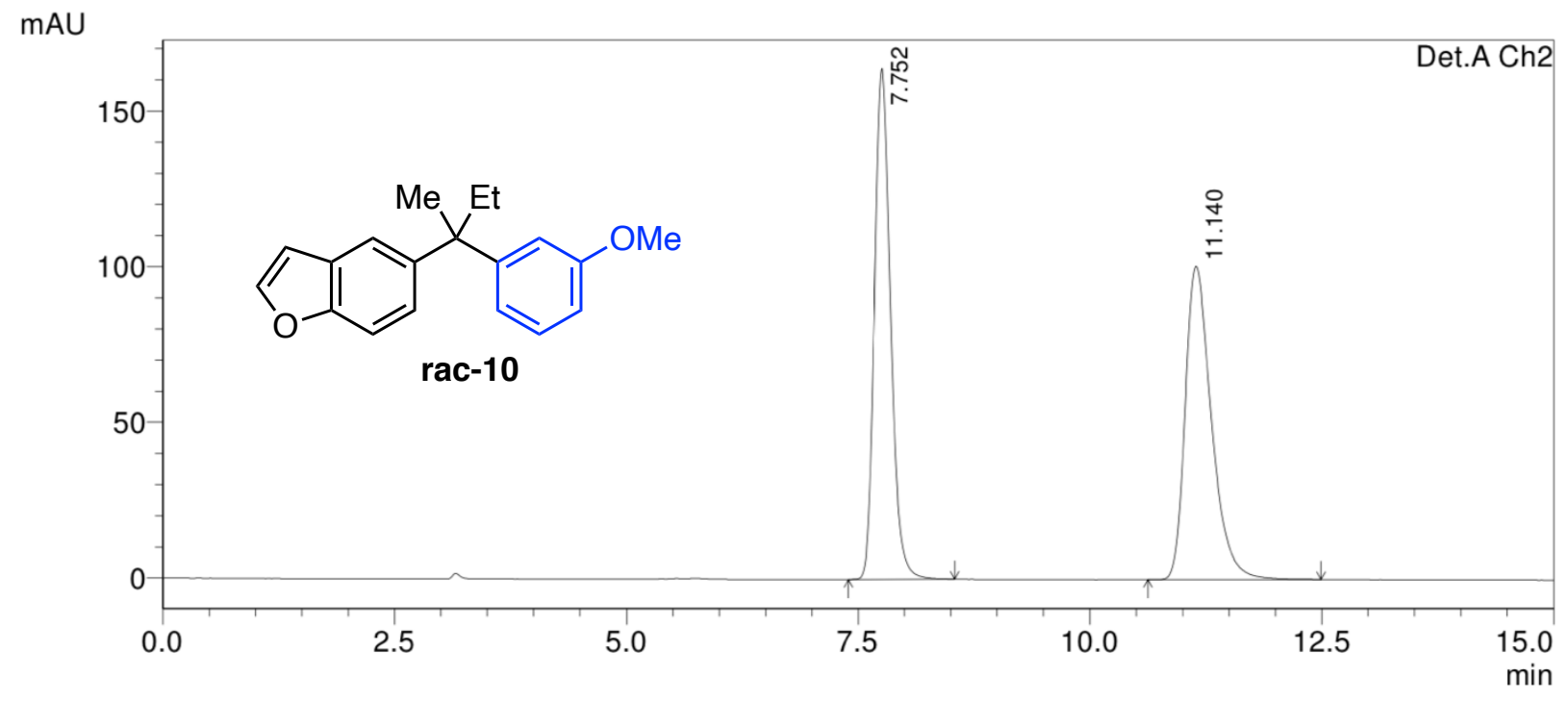

Detector A Ch2 220nm

\begin{tabular}{|r|r|r|r|r|r|}
\hline \multicolumn{1}{|c|}{ Peak\# } & Ret. Time & \multicolumn{1}{|c|}{ Area } & Height & Area \% & Height \% \\
\hline 1 & 7.752 & 1989174 & 164076 & 50.052 & 61.981 \\
\hline 2 & 11.140 & 1985025 & 100642 & 49.948 & 38.019 \\
\hline Total & & 3974199 & 264719 & 100.000 & 100.000 \\
\hline
\end{tabular}

mAU

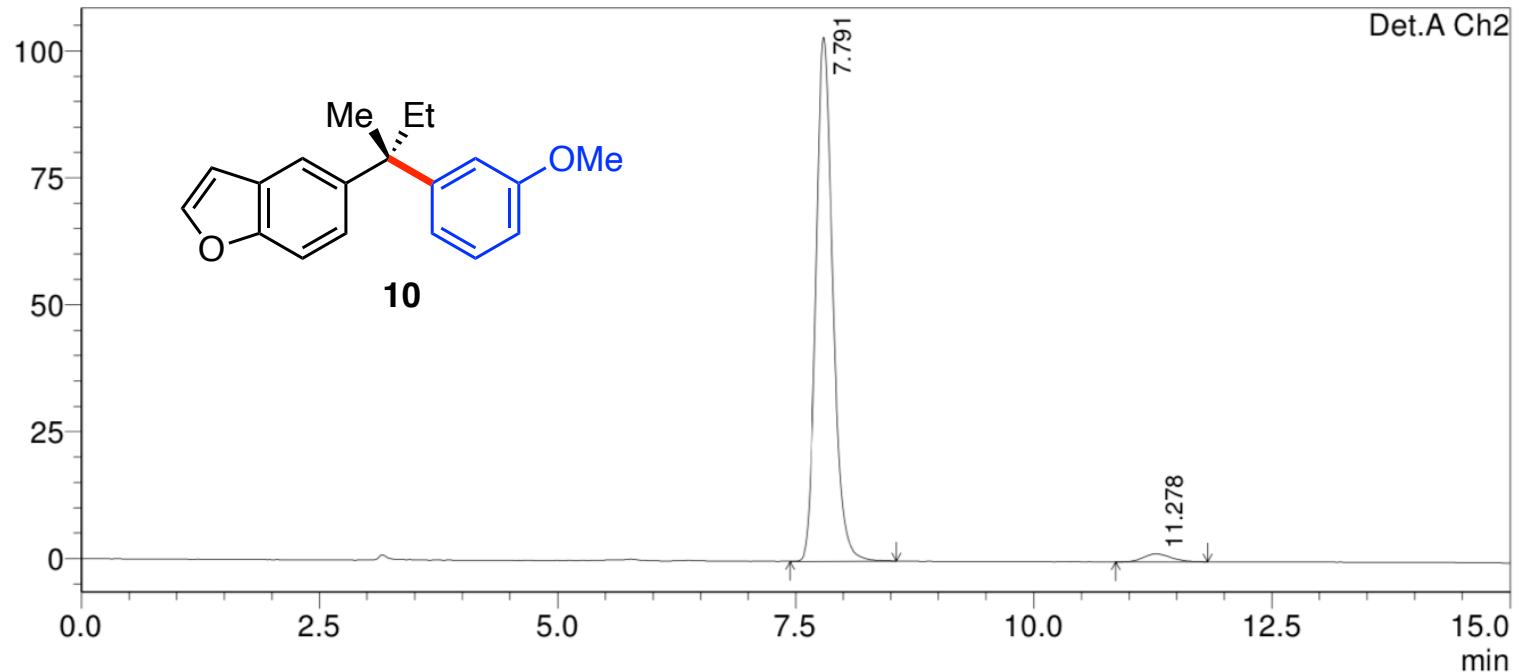

Detector A Ch2 220nm

\begin{tabular}{|r|r|r|r|r|r|}
\hline \multicolumn{1}{|c|}{ Peak\# } & Ret. Time & \multicolumn{1}{c|}{ Area } & Height & Area \% & Height \% \\
\hline 1 & 7.791 & 1277822 & 103198 & 97.540 & 98.493 \\
\hline 2 & 11.278 & 32222 & 1579 & 2.460 & 1.507 \\
\hline Total & & 1310044 & 104777 & 100.000 & 100.000 \\
\hline
\end{tabular}




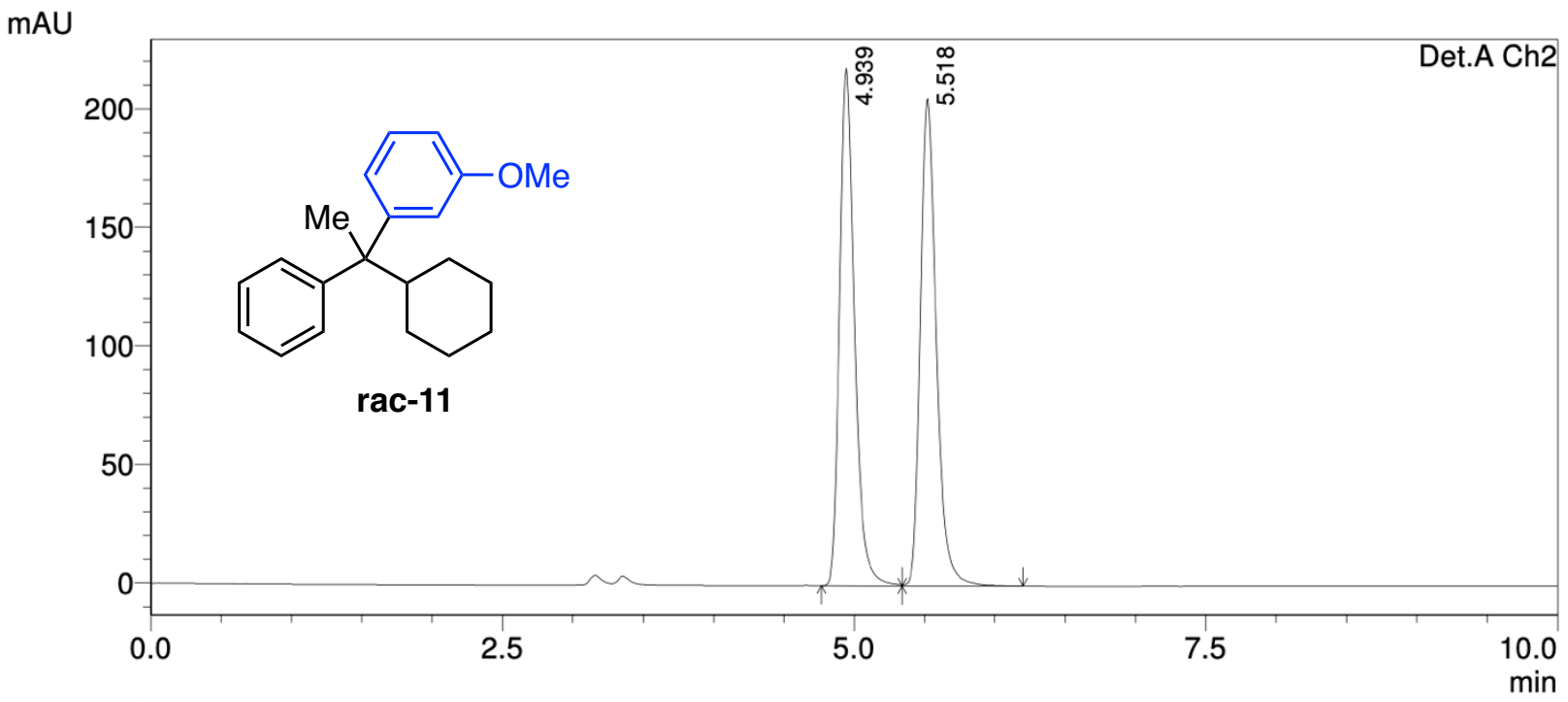

Detector A Ch2 220nm

\begin{tabular}{|r|r|r|r|r|r|}
\hline \multicolumn{1}{|c|}{ Peak\# } & Ret. Time & Area & Height & \multicolumn{1}{|c|}{ Area \% } & \multicolumn{1}{|c|}{ Height \% } \\
\hline 1 & 4.939 & 1615570 & 218170 & 49.843 & 51.511 \\
\hline 2 & 5.518 & 1625771 & 205372 & 50.157 & 48.489 \\
\hline Total & & 3241341 & 423542 & 100.000 & 100.000 \\
\hline
\end{tabular}

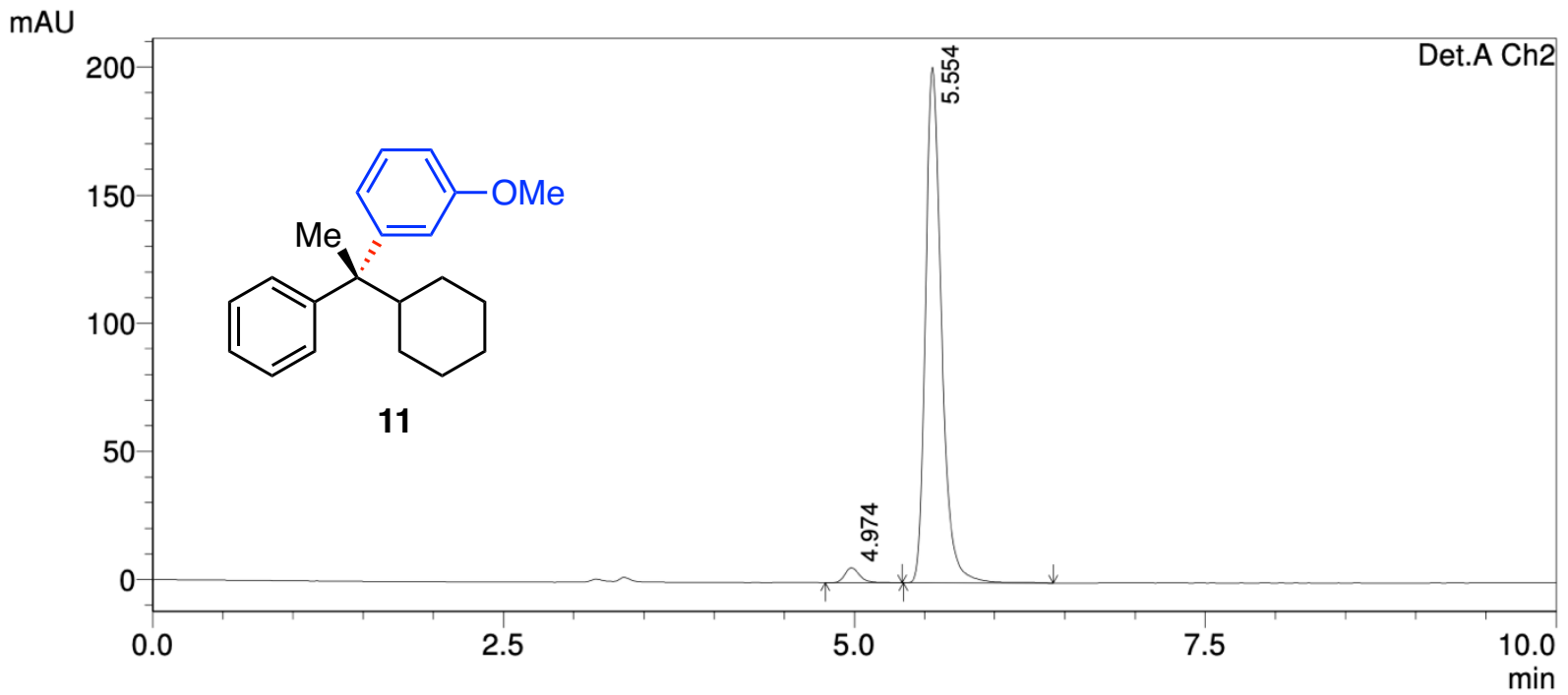

Detector A Ch2 220nm

\begin{tabular}{|r|r|r|r|r|r|}
\hline \multicolumn{1}{|c|}{ Peak\# } & Ret. Time & \multicolumn{1}{c|}{ Area } & Height & Area \% & \multicolumn{1}{|c|}{ Height \% } \\
\hline 1 & 4.974 & 42735 & 5813 & 2.609 & 2.808 \\
\hline 2 & 5.554 & 1595090 & 201218 & 97.391 & 97.192 \\
\hline Total & & 1637824 & 207030 & 100.000 & 100.000 \\
\hline
\end{tabular}




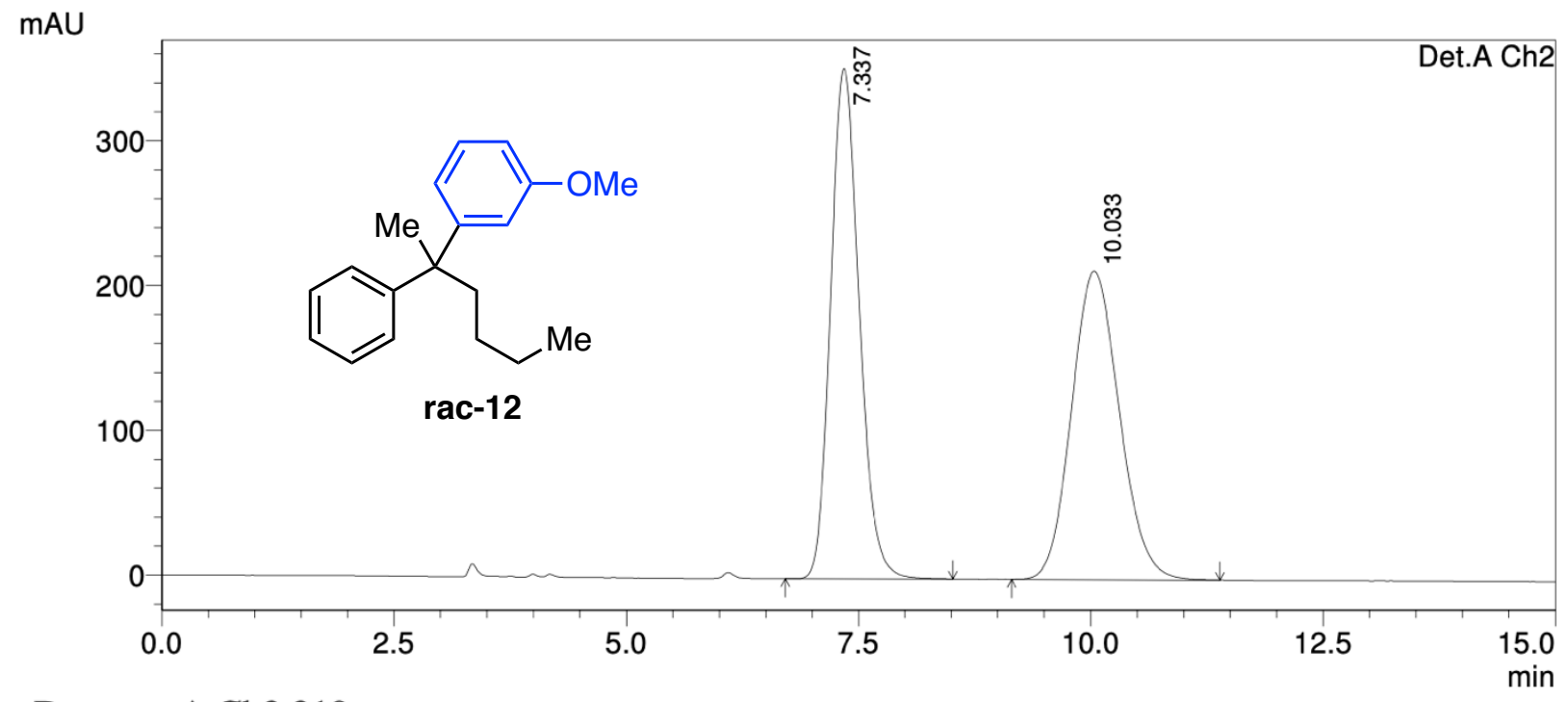

Detector A Ch2 210nm

\begin{tabular}{|r|r|r|r|r|r|}
\hline \multicolumn{1}{|c|}{ Peak\# } & Ret. Time & \multicolumn{1}{|c|}{ Area } & Height & Area \% & \multicolumn{1}{|c|}{ Height \% } \\
\hline 1 & 7.337 & 7555214 & 352426 & 49.775 & 62.334 \\
\hline 2 & 10.033 & 7623569 & 212957 & 50.225 & 37.666 \\
\hline Total & & 15178783 & 565383 & 100.000 & 100.000 \\
\hline
\end{tabular}

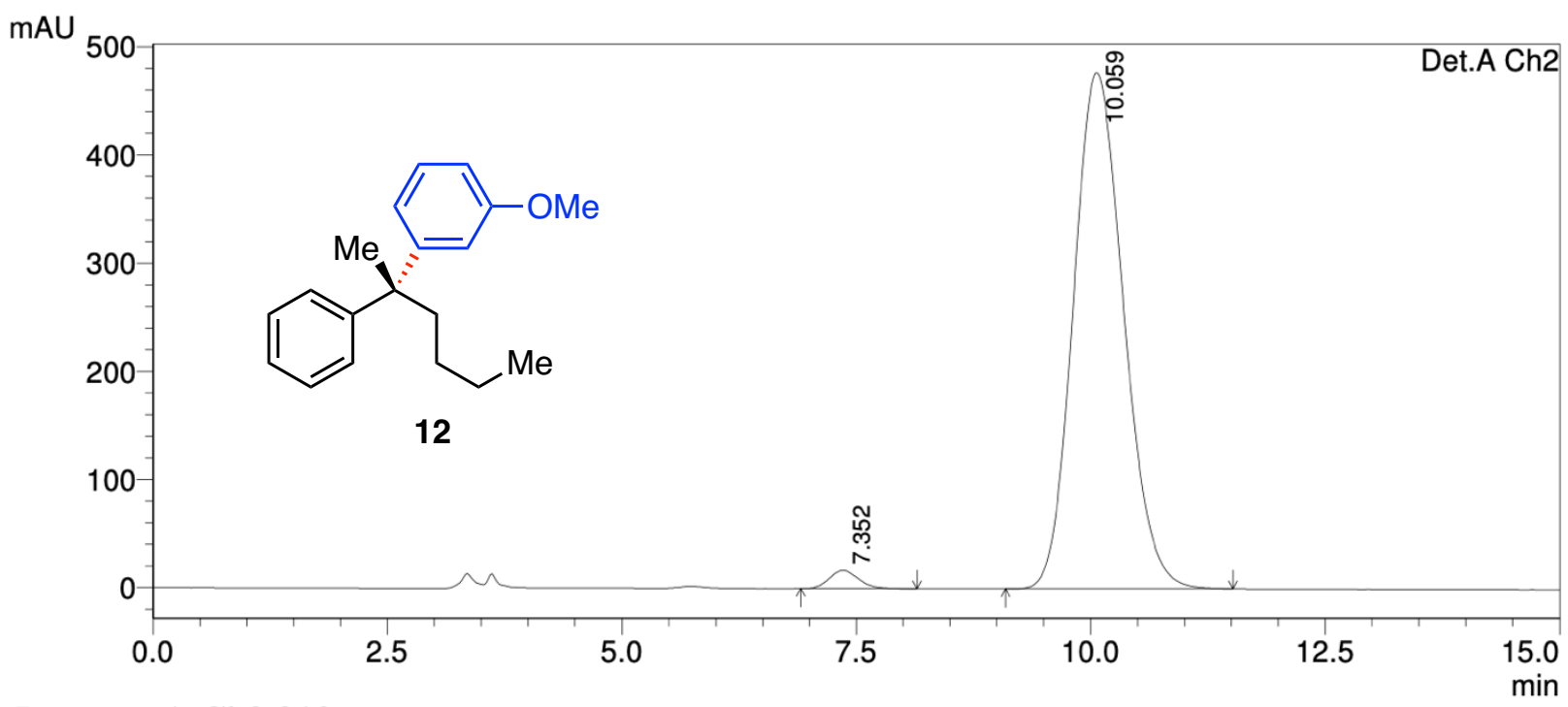

Detector A Ch2 210nm

\begin{tabular}{|r|r|r|r|r|r|}
\hline \multicolumn{1}{|c|}{ Peak\# } & Ret. Time & \multicolumn{1}{|c|}{ Area } & Height & Area \% & \multicolumn{1}{|c|}{ Height \% } \\
\hline 1 & 7.352 & 365948 & 16878 & 2.020 & 3.417 \\
\hline 2 & 10.059 & 17752610 & 477011 & 97.980 & 96.583 \\
\hline Total & & 18118559 & 493890 & 100.000 & 100.000 \\
\hline
\end{tabular}




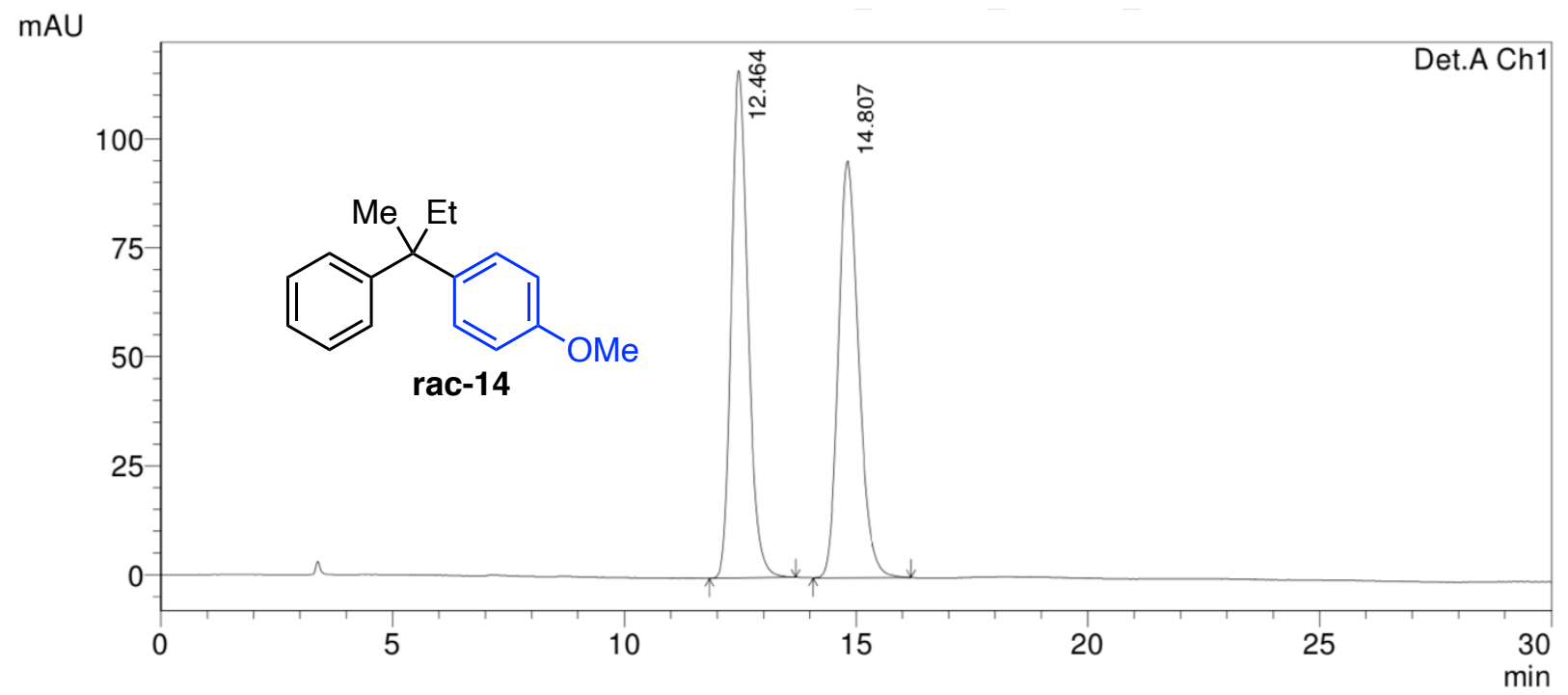

Detector A Ch1 220nm

\begin{tabular}{|r|r|r|r|r|r|}
\hline \multicolumn{1}{|c|}{ Peak\# } & Ret. Time & \multicolumn{1}{c|}{ Area } & \multicolumn{1}{c|}{ Height } & Area \% & \multicolumn{1}{c|}{ Height \% } \\
\hline 1 & 12.464 & 2912438 & 116346 & 49.945 & 54.893 \\
\hline 2 & 14.807 & 2918845 & 95604 & 50.055 & 45.107 \\
\hline Total & & 5831283 & 211949 & 100.000 & 100.000 \\
\hline
\end{tabular}

mAU

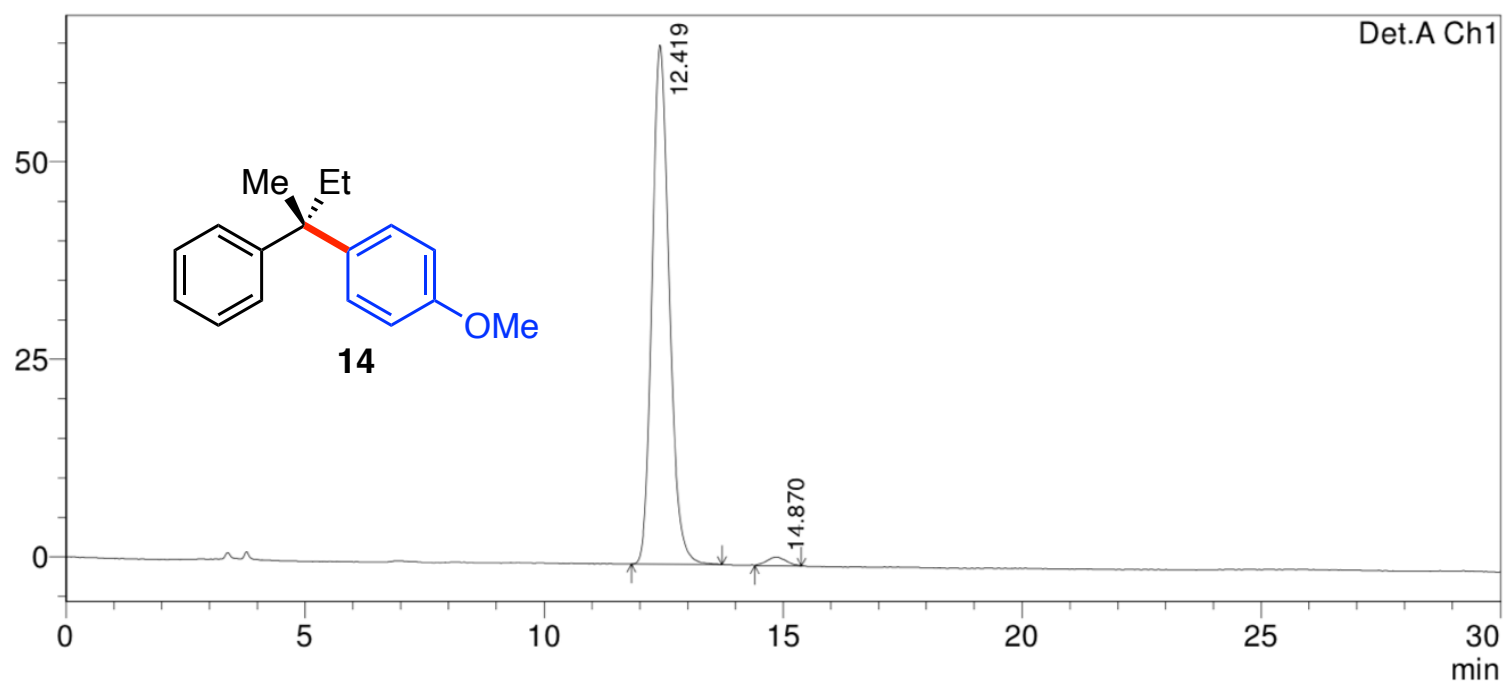

\begin{tabular}{|r|r|r|r|r|r|}
\hline \multicolumn{1}{|c|}{ Peak\# } & Ret. Time & \multicolumn{1}{c|}{ Area } & Height & Area \% & \multicolumn{1}{|c|}{ Height \% } \\
\hline 1 & 12.419 & 1649310 & 65709 & 98.152 & 98.350 \\
\hline 2 & 14.870 & 31050 & 1103 & 1.848 & 1.650 \\
\hline Total & & 1680360 & 66811 & 100.000 & 100.000 \\
\hline
\end{tabular}




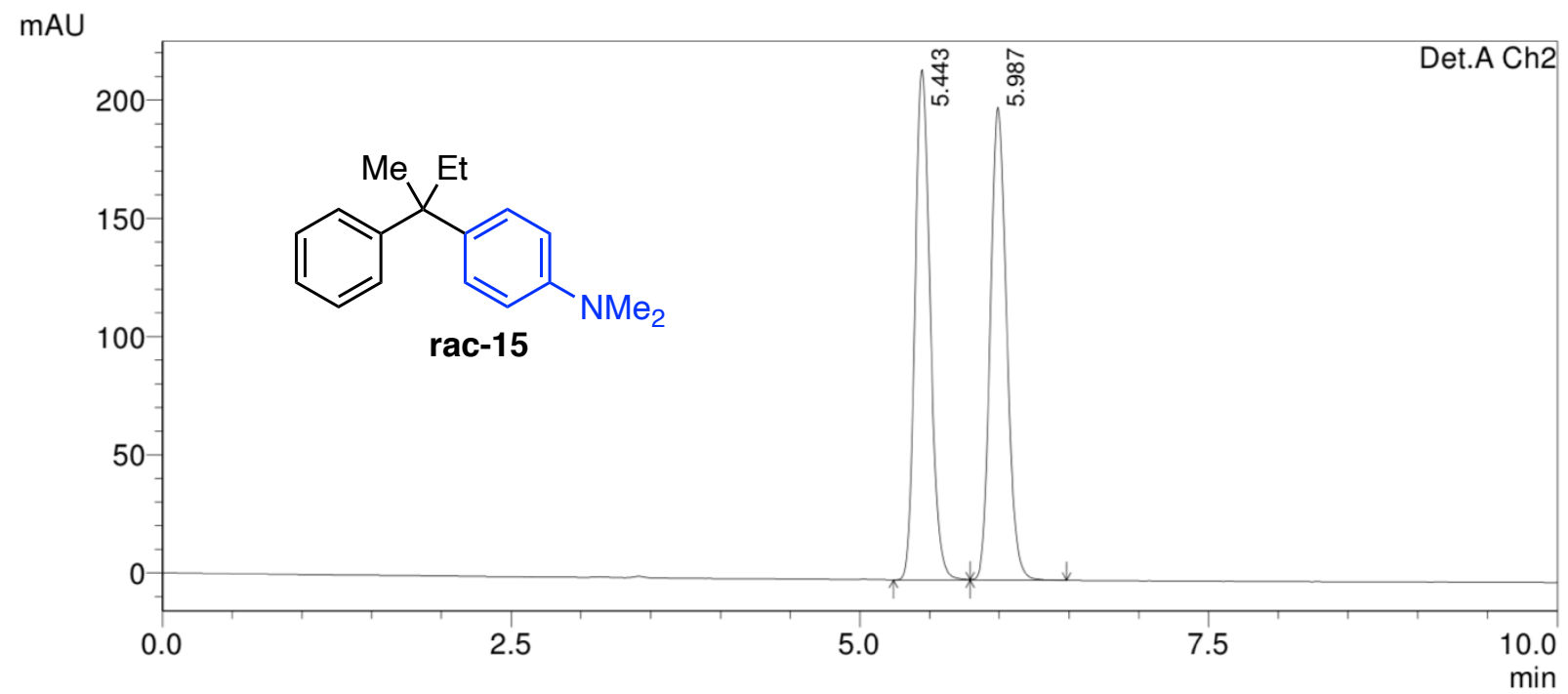

Detector A Ch2 254nm

\begin{tabular}{|r|r|r|r|r|r|}
\hline \multicolumn{1}{|c|}{ Peak\# } & Ret. Time & \multicolumn{1}{|c|}{ Area } & Height & Area \% & \multicolumn{1}{|c|}{ Height \% } \\
\hline 1 & 5.443 & 1633079 & 215729 & 49.985 & 51.882 \\
\hline 2 & 5.987 & 1634077 & 200075 & 50.015 & 48.118 \\
\hline Total & & 3267157 & 415804 & 100.000 & 100.000 \\
\hline
\end{tabular}

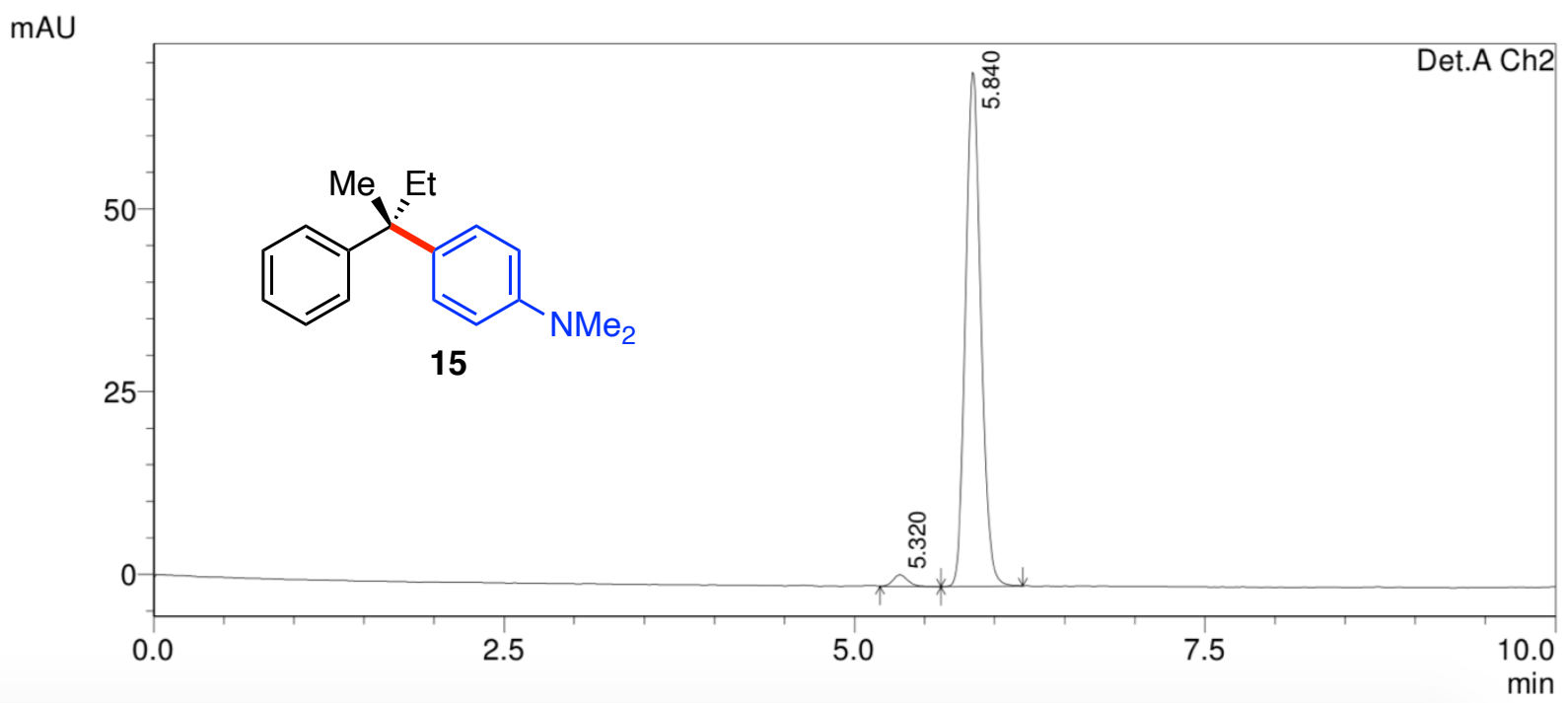

Detector A Ch2 254nm

\begin{tabular}{|r|r|r|r|r|r|}
\hline \multicolumn{1}{|c|}{ Peak\# } & Ret. Time & \multicolumn{1}{c|}{ Area } & Height & Area \% & \multicolumn{1}{|c|}{ Height \% } \\
\hline 1 & 5.320 & 11785 & 1570 & 2.148 & 2.185 \\
\hline 2 & 5.840 & 536861 & 70288 & 97.852 & 97.815 \\
\hline Total & & 548645 & 71857 & 100.000 & 100.000 \\
\hline
\end{tabular}




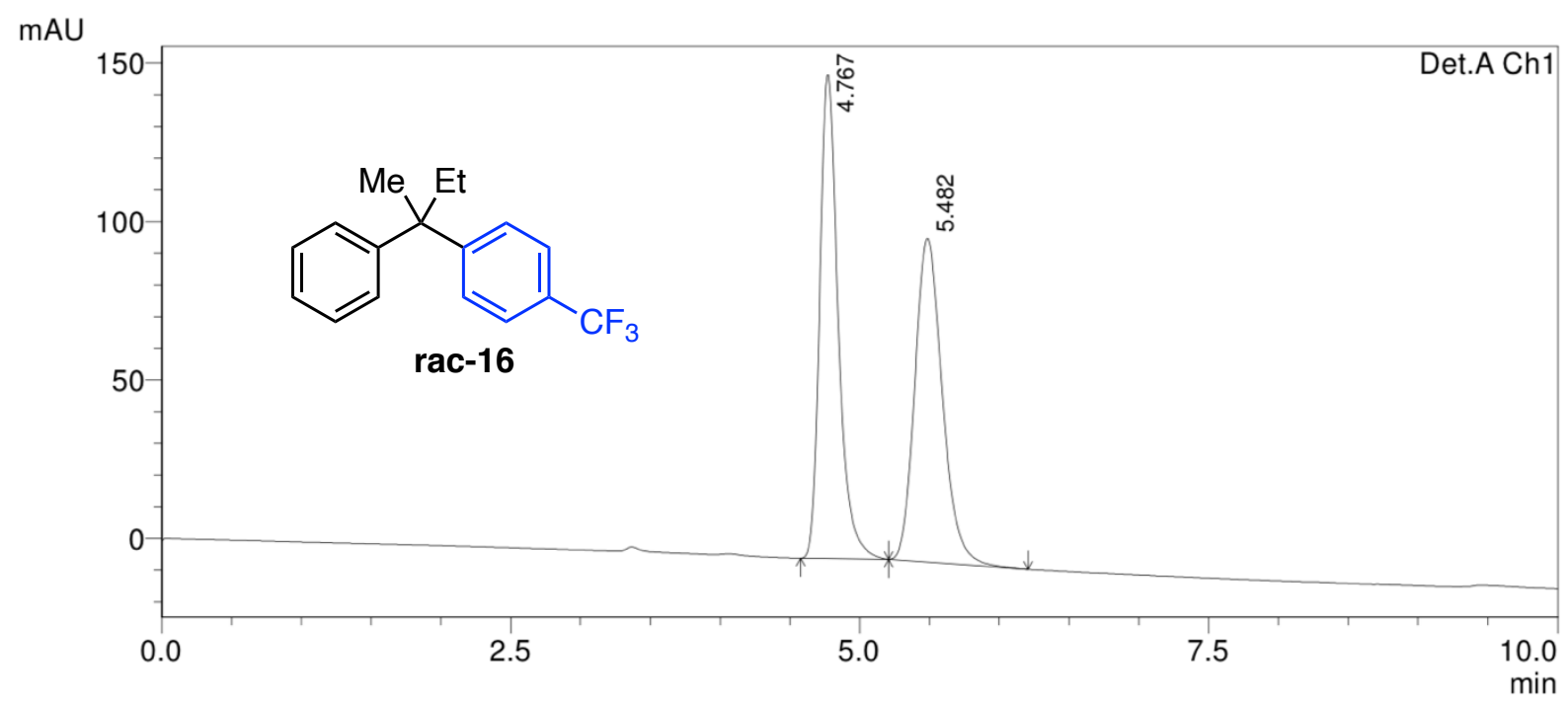

Detector A Ch1 220nm

\begin{tabular}{|r|r|r|r|r|r|}
\hline \multicolumn{1}{|c|}{ Peak\# } & Ret. Time & Area & Height & Area \% & \multicolumn{1}{|c|}{ Height \% } \\
\hline 1 & 4.767 & 1402214 & 152583 & 50.054 & 59.908 \\
\hline 2 & 5.482 & 1399182 & 102111 & 49.946 & 40.092 \\
\hline Total & & 2801396 & 254693 & 100.000 & 100.000 \\
\hline
\end{tabular}

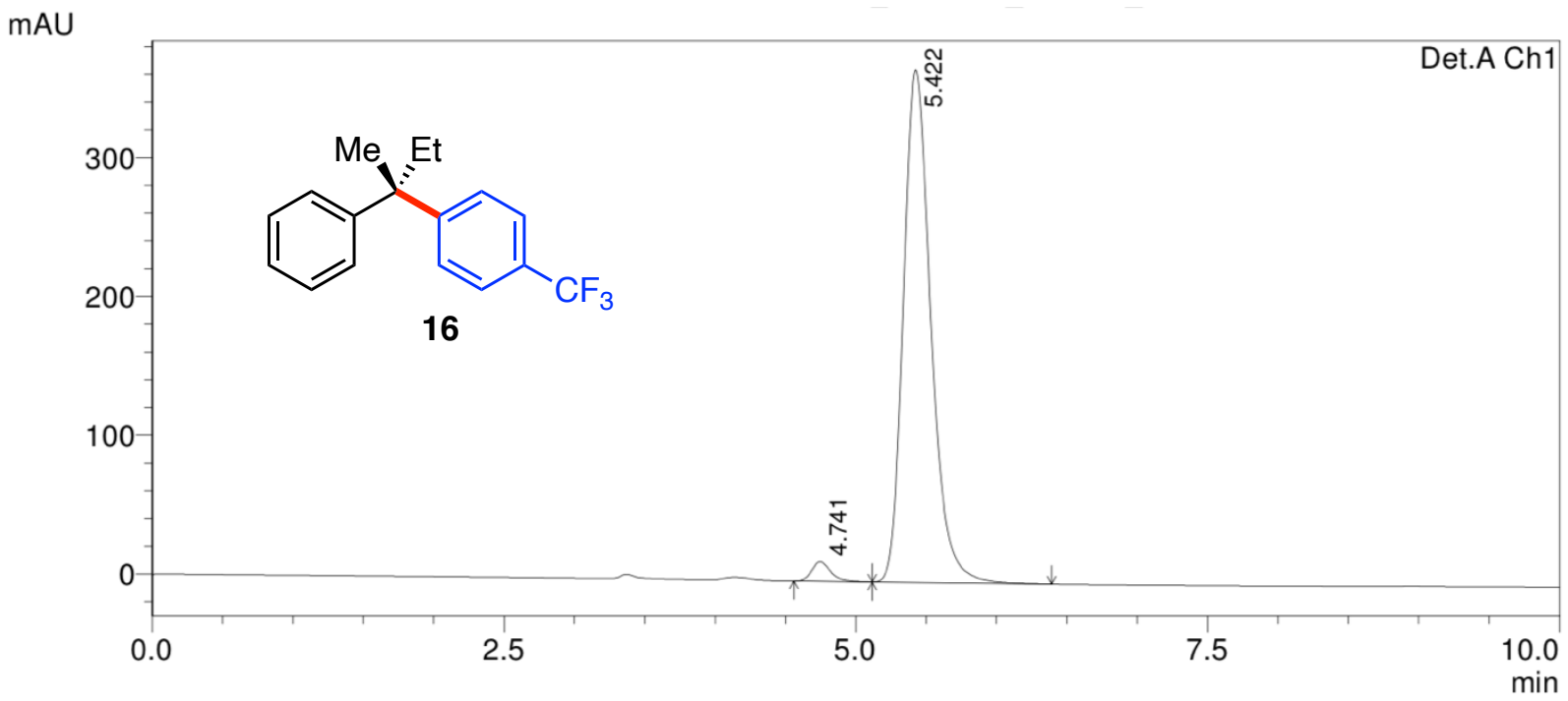

\begin{tabular}{|r|r|r|r|r|r|}
\hline \multicolumn{1}{|c|}{ Peak\# } & Ret. Time & \multicolumn{1}{c|}{ Area } & Height & Area \% & \multicolumn{1}{c|}{ Height \% } \\
\hline 1 & 4.741 & 131902 & 14075 & 2.566 & 3.670 \\
\hline 2 & 5.422 & 5009433 & 369443 & 97.434 & 96.330 \\
\hline Total & & 5141335 & 383518 & 100.000 & 100.000 \\
\hline
\end{tabular}




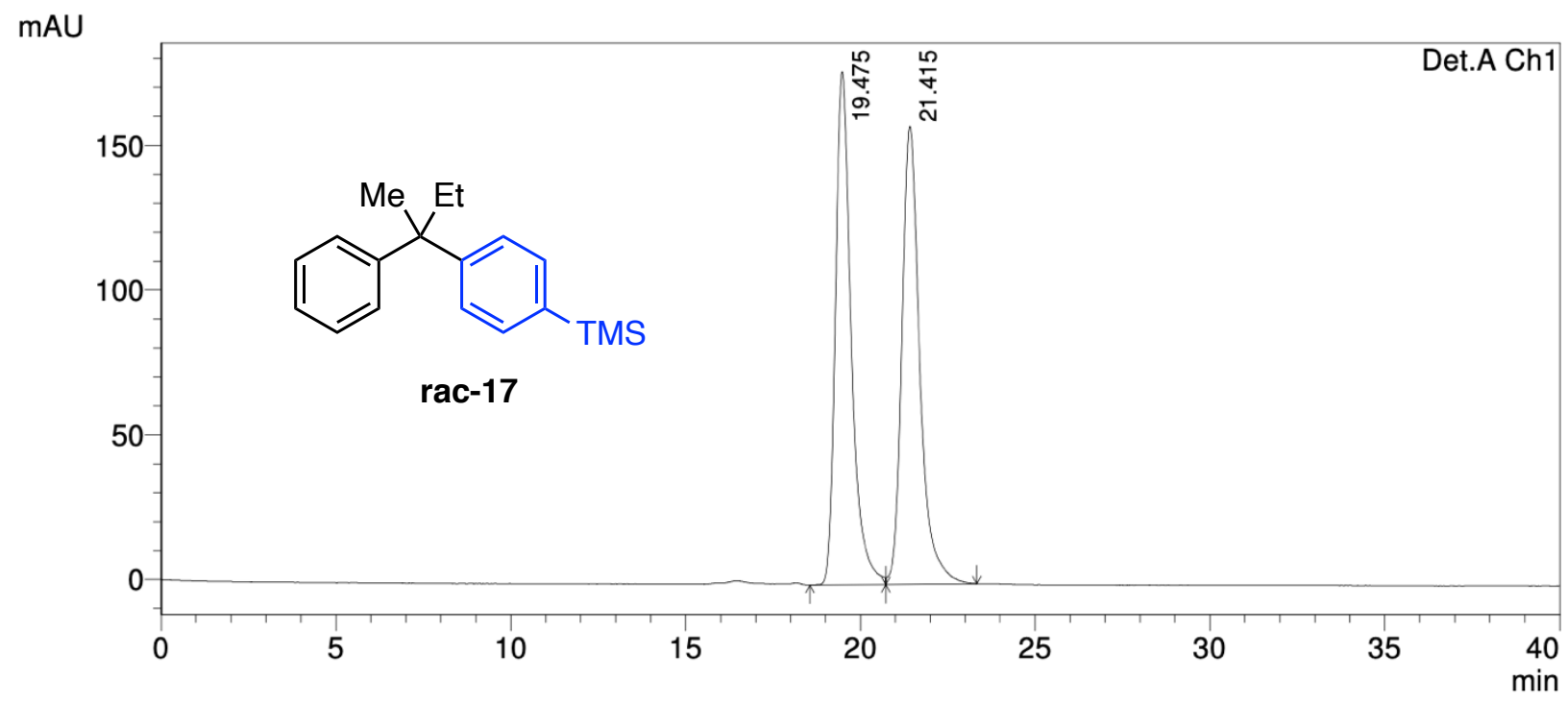

Detector A Ch1 220nm

\begin{tabular}{|r|r|r|r|r|r|}
\hline Peak\# & Ret. Time & \multicolumn{1}{|c|}{ Area } & Height & Area \% & \multicolumn{1}{|c|}{ Height \% } \\
\hline 1 & 19.475 & 5570283 & 177204 & 49.719 & 52.834 \\
\hline 2 & 21.415 & 5633230 & 158193 & 50.281 & 47.166 \\
\hline Total & & 11203513 & 335397 & 100.000 & 100.000 \\
\hline
\end{tabular}

mAU

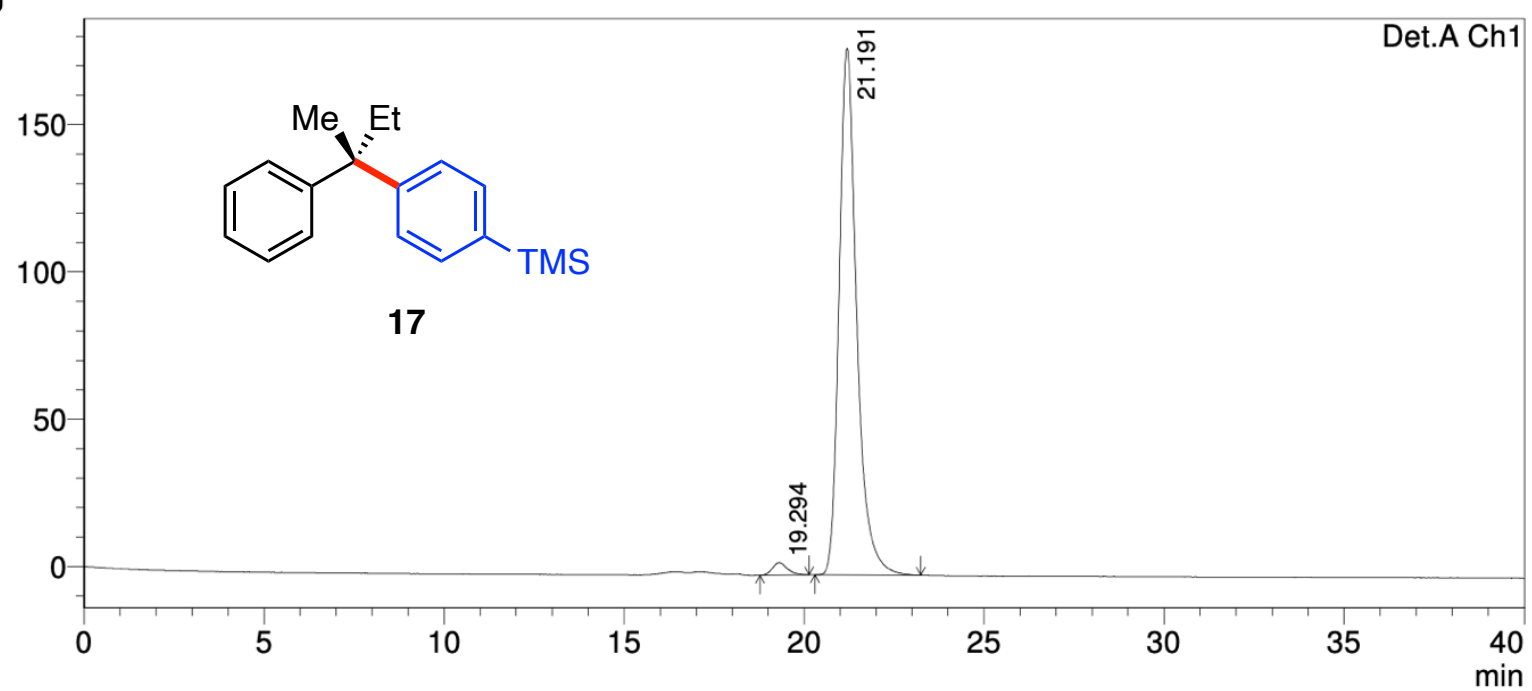

Detector A Ch1 220nm

\begin{tabular}{|r|r|r|r|r|r|}
\hline Peak\# & Ret. Time & \multicolumn{1}{|c|}{ Area } & \multicolumn{1}{|c|}{ Height } & Area \% & \multicolumn{1}{c|}{ Height \% } \\
\hline 1 & 19.294 & 124032 & 4268 & 1.991 & 2.331 \\
\hline 2 & 21.191 & 6106556 & 178849 & 98.009 & 97.669 \\
\hline Total & & 6230588 & 183117 & 100.000 & 100.000 \\
\hline
\end{tabular}




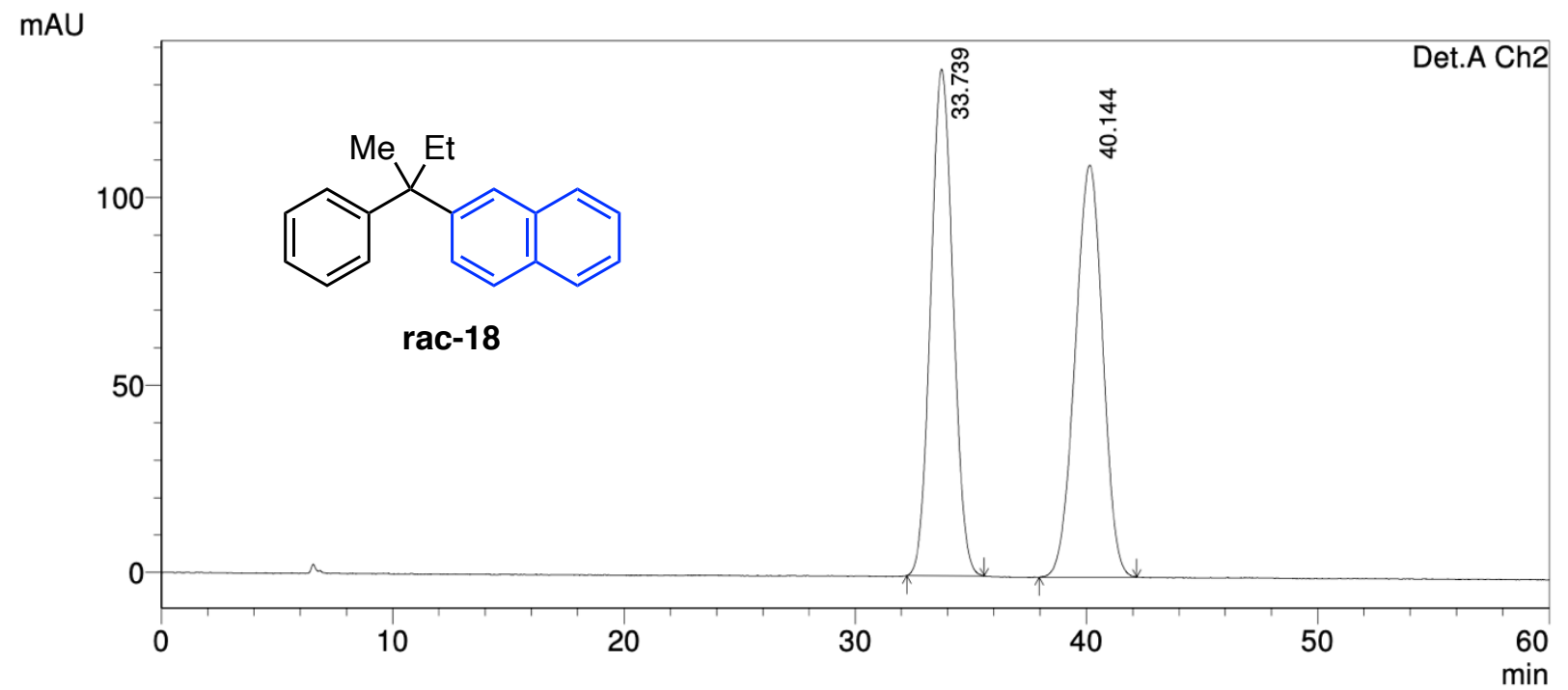

Detector A Ch2 210nm

\begin{tabular}{|r|r|r|r|r|r|}
\hline Peak\# & Ret. Time & \multicolumn{1}{c|}{ Area } & Height & Area \% & \multicolumn{1}{|c|}{ Height \% } \\
\hline 1 & 33.739 & 9094304 & 135109 & 49.927 & 55.155 \\
\hline 2 & 40.144 & 9121017 & 109855 & 50.073 & 44.845 \\
\hline Total & & 18215321 & 244964 & 100.000 & 100.000 \\
\hline
\end{tabular}

mAU

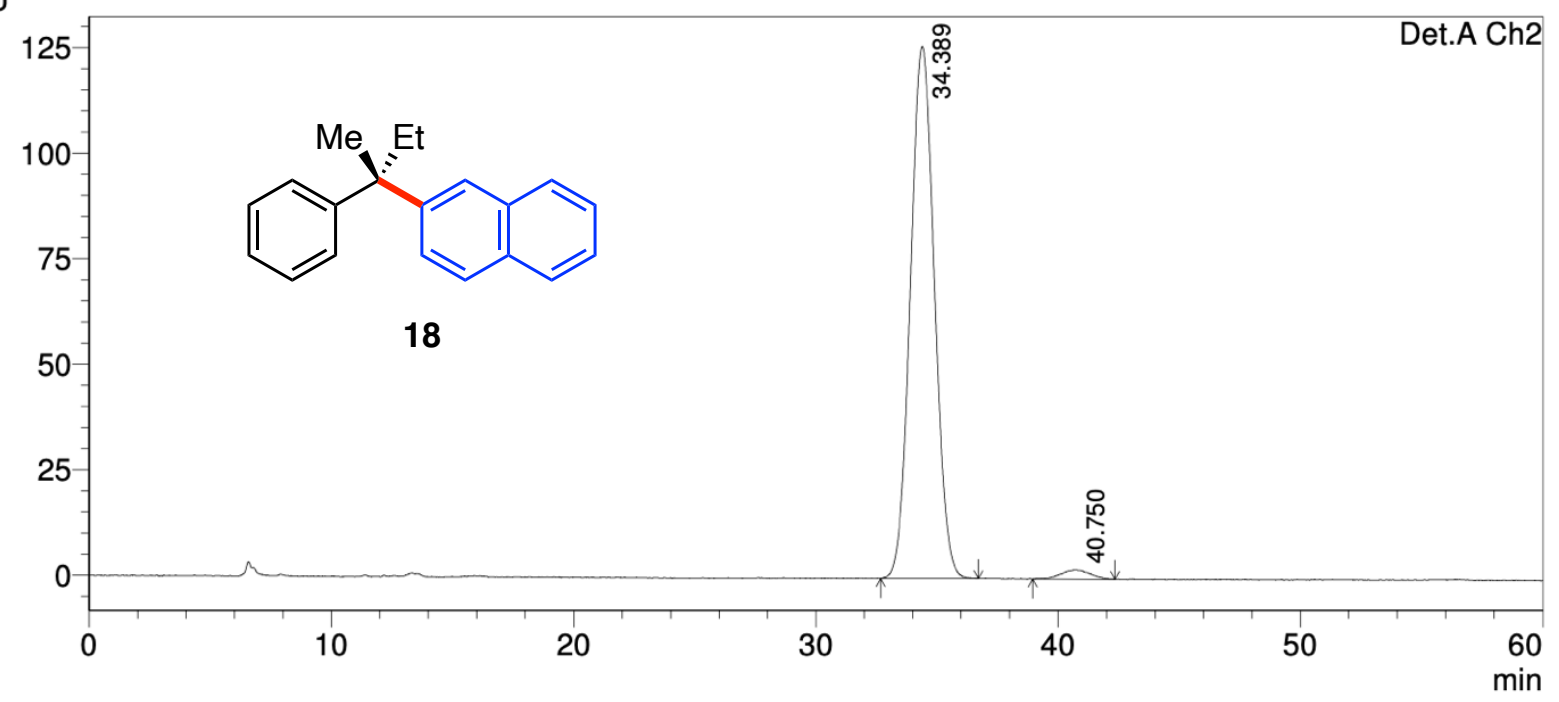

Detector A Ch2 210nm

\begin{tabular}{|r|r|r|r|r|r|}
\hline \multicolumn{1}{|c|}{ Peak\# } & Ret. Time & \multicolumn{1}{|c|}{ Area } & Height & Area \% & Height \% \\
\hline 1 & 34.389 & 8676167 & 125961 & 97.933 & 98.272 \\
\hline 2 & 40.750 & 183164 & 2215 & 2.067 & 1.728 \\
\hline Total & & 8859331 & 128175 & 100.000 & 100.000 \\
\hline
\end{tabular}




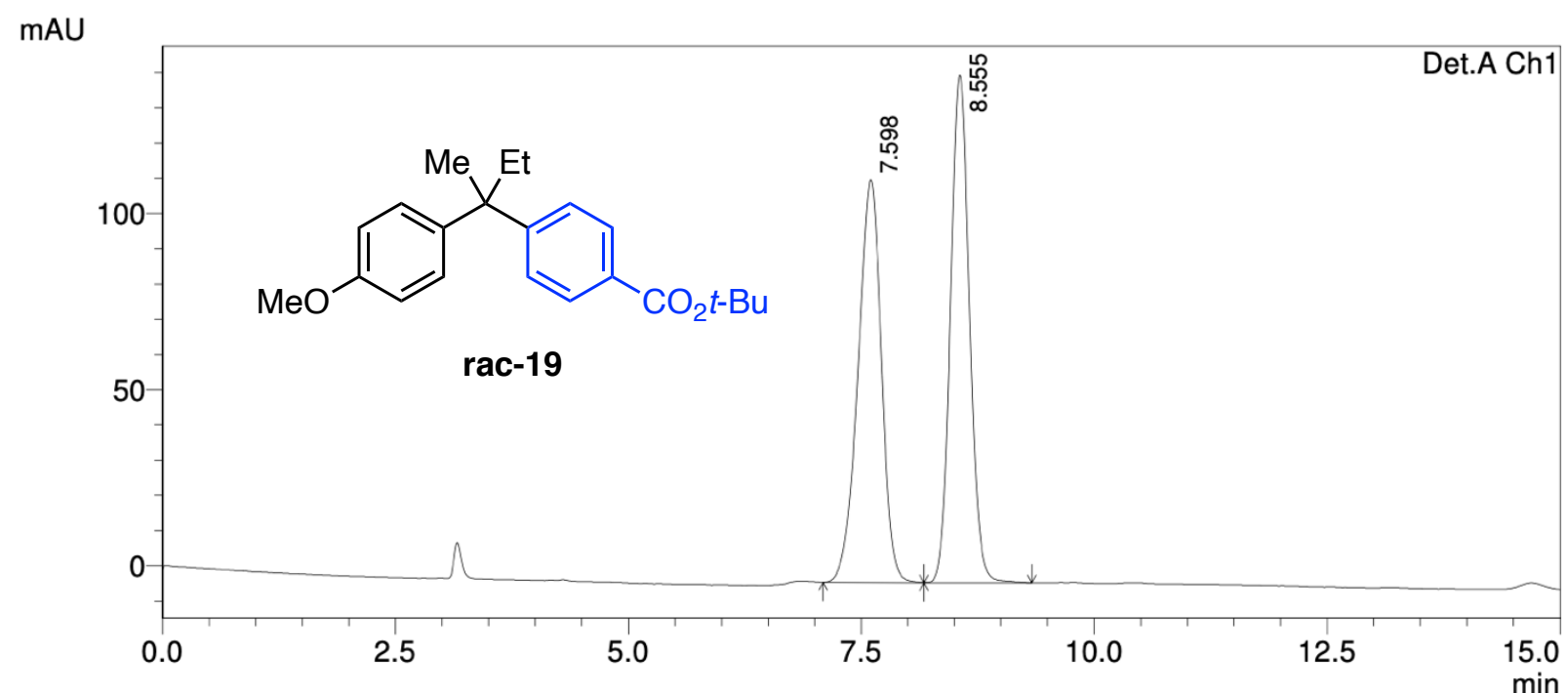

\begin{tabular}{|r|r|r|r|r|r|}
\hline Peak\# & Ret. Time & Area & Height & Area \% & \multicolumn{1}{|c|}{ Height \% } \\
\hline 1 & 7.598 & 2015789 & 114362 & 49.693 & 44.241 \\
\hline 2 & 8.555 & 2040705 & 144137 & 50.307 & 55.759 \\
\hline Total & & 4056494 & 258498 & 100.000 & 100.000 \\
\hline
\end{tabular}

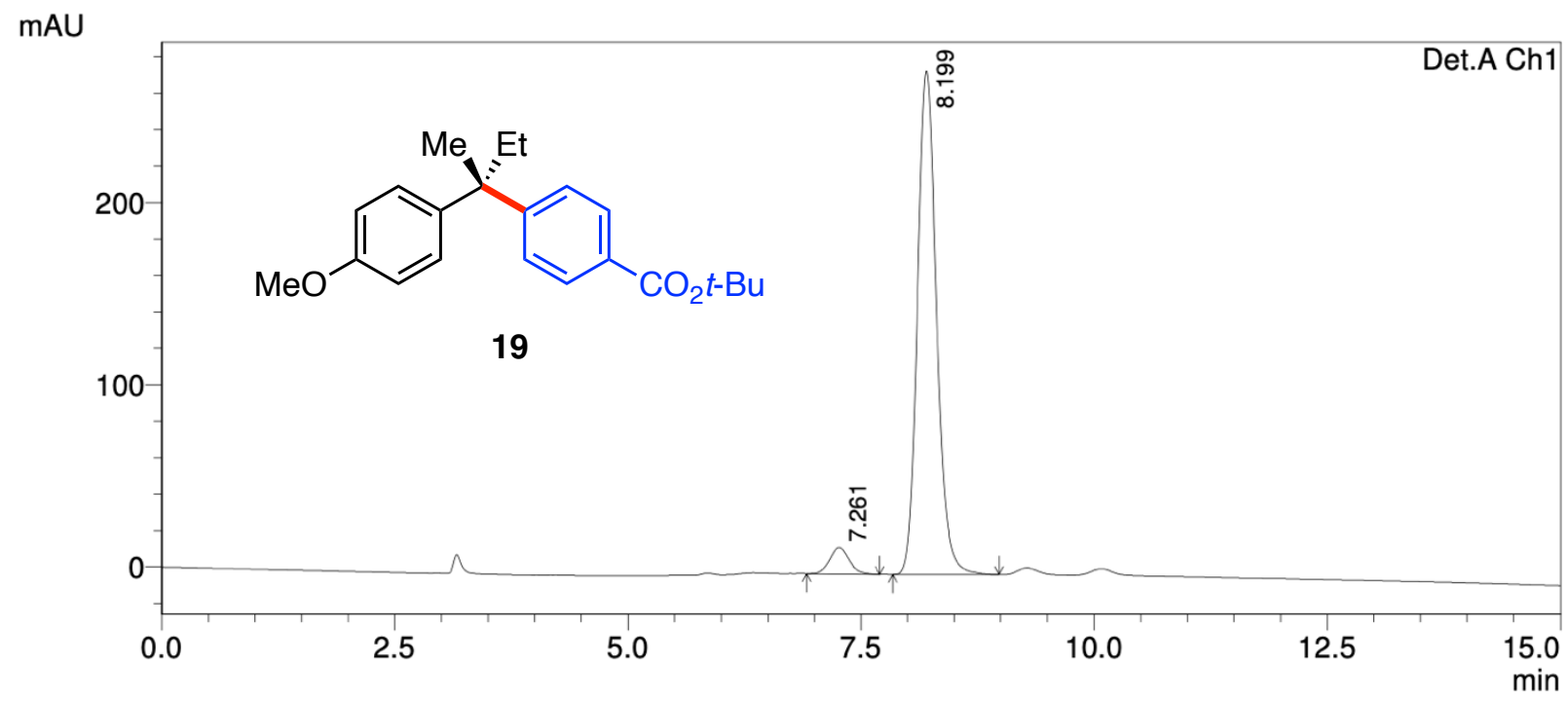

Detector A Ch1 210nm

\begin{tabular}{|r|r|r|r|r|r|}
\hline Peak\# & Ret. Time & \multicolumn{1}{|c|}{ Area } & Height & Area \% & Height \% \\
\hline 1 & 7.261 & 209156 & 14542 & 5.059 & 5.002 \\
\hline 2 & 8.199 & 3925137 & 276158 & 94.941 & 94.998 \\
\hline Total & & 4134293 & 290700 & 100.000 & 100.000 \\
\hline
\end{tabular}




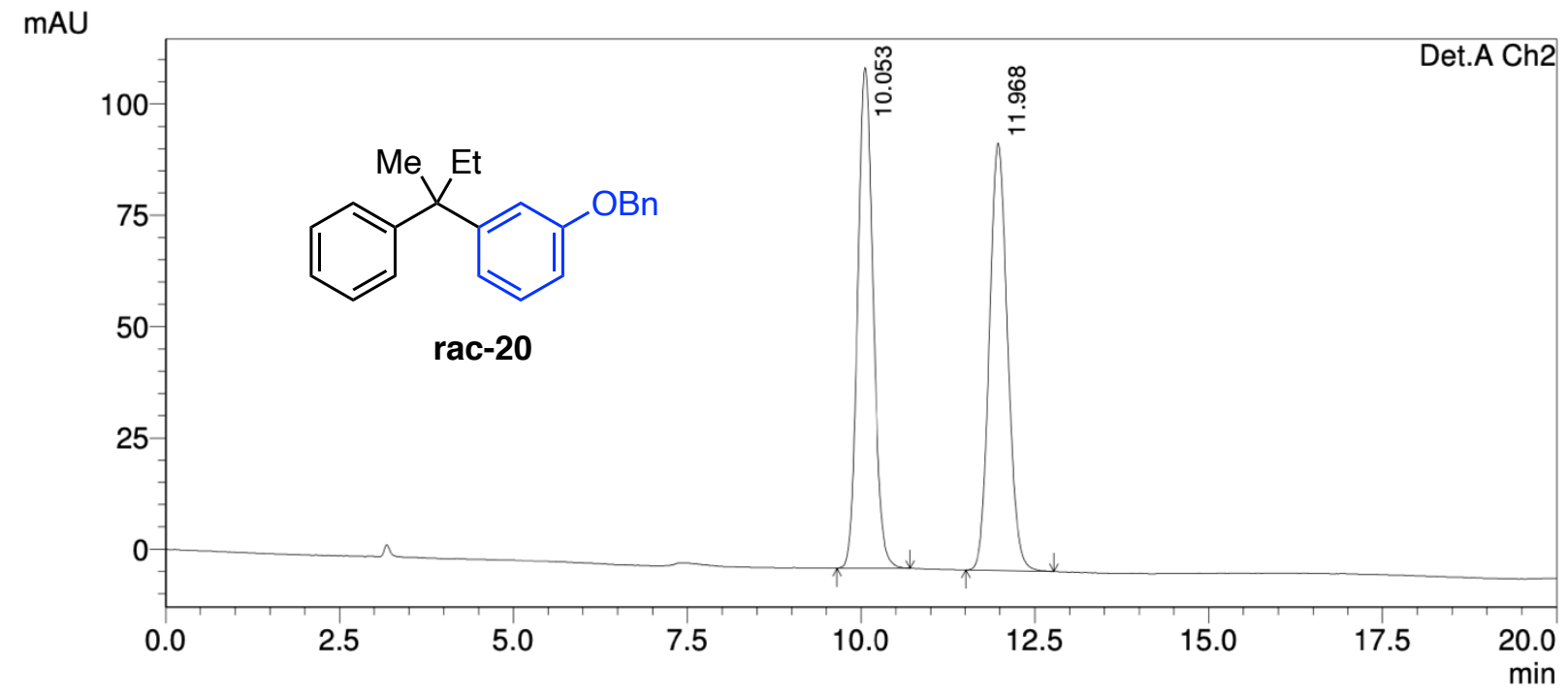

Detector A Ch2 210nm

\begin{tabular}{|r|r|r|r|r|r|}
\hline \multicolumn{1}{|c|}{ Peak\# } & Ret. Time & Area & Height & Area \% & Height \% \\
\hline 1 & 10.053 & 1747731 & 112468 & 49.922 & 53.935 \\
\hline 2 & 11.968 & 1753161 & 96056 & 50.078 & 46.065 \\
\hline Total & & 3500892 & 208524 & 100.000 & 100.000 \\
\hline
\end{tabular}

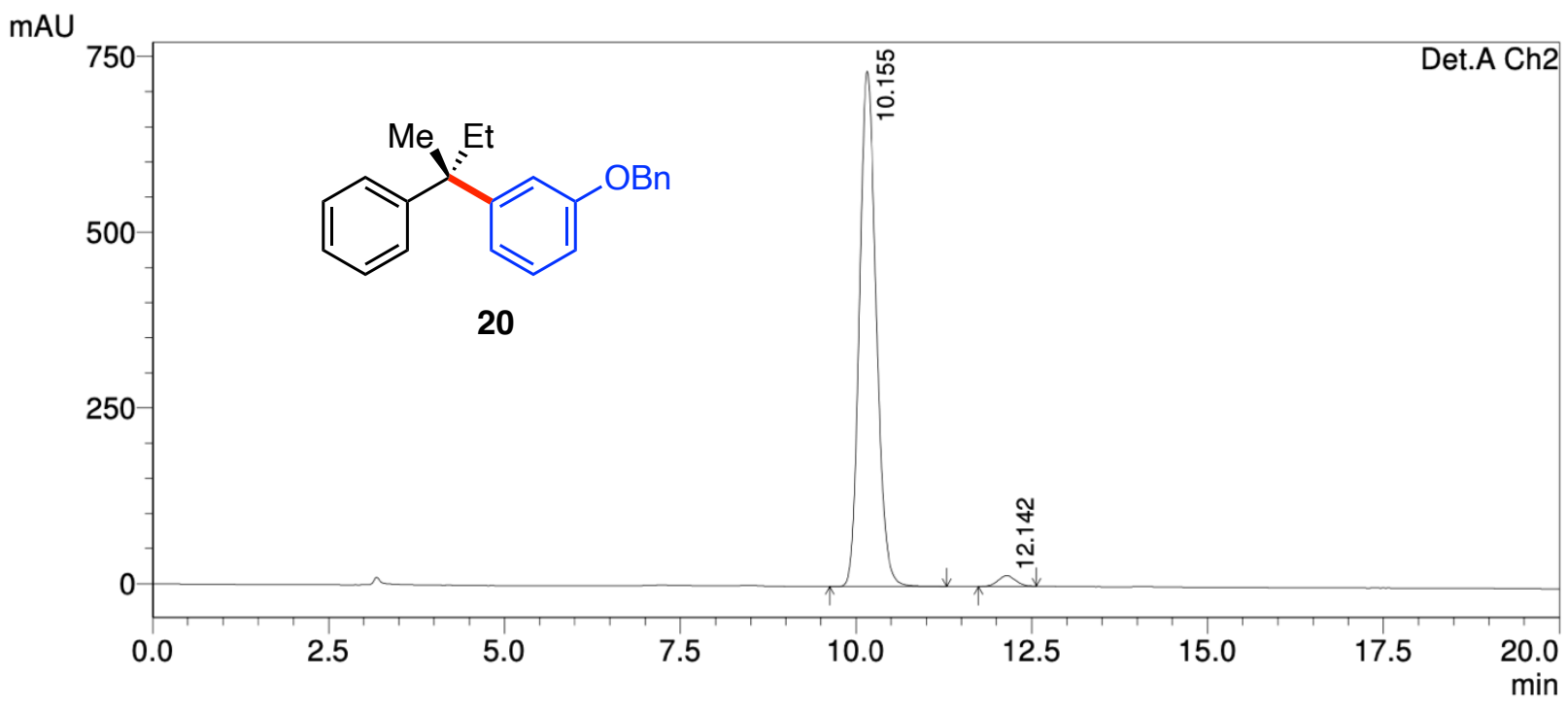

Detector A Ch2 210nm

\begin{tabular}{|r|r|r|r|r|r|}
\hline \multicolumn{1}{|c|}{ Peak\# } & Ret. Time & \multicolumn{1}{|c|}{ Area } & Height & Area \% & Height \% \\
\hline 1 & 10.155 & 12327552 & 732973 & 97.929 & 98.005 \\
\hline 2 & 12.142 & 260709 & 14918 & 2.071 & 1.995 \\
\hline Total & & 12588261 & 747892 & 100.000 & 100.000 \\
\hline
\end{tabular}




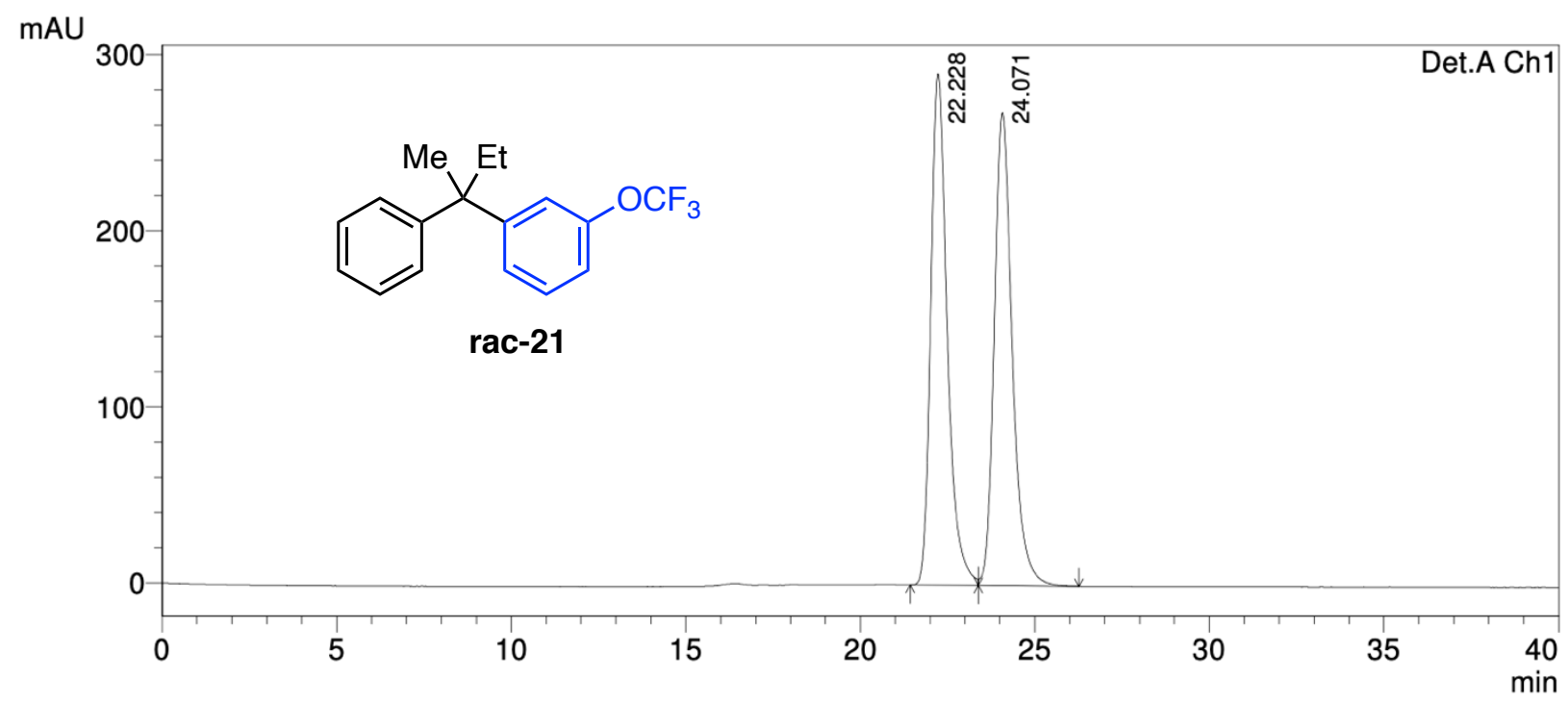

Detector A Ch1 220nm

\begin{tabular}{|r|r|r|r|r|r|}
\hline \multicolumn{1}{|c|}{ Peak\# } & Ret. Time & \multicolumn{1}{|c|}{ Area } & Height & Area \% & \multicolumn{1}{|c|}{ Height \% } \\
\hline 1 & 22.228 & 9529988 & 290436 & 49.718 & 51.953 \\
\hline 2 & 24.071 & 9638060 & 268600 & 50.282 & 48.047 \\
\hline Total & & 19168048 & 559036 & 100.000 & 100.000 \\
\hline
\end{tabular}

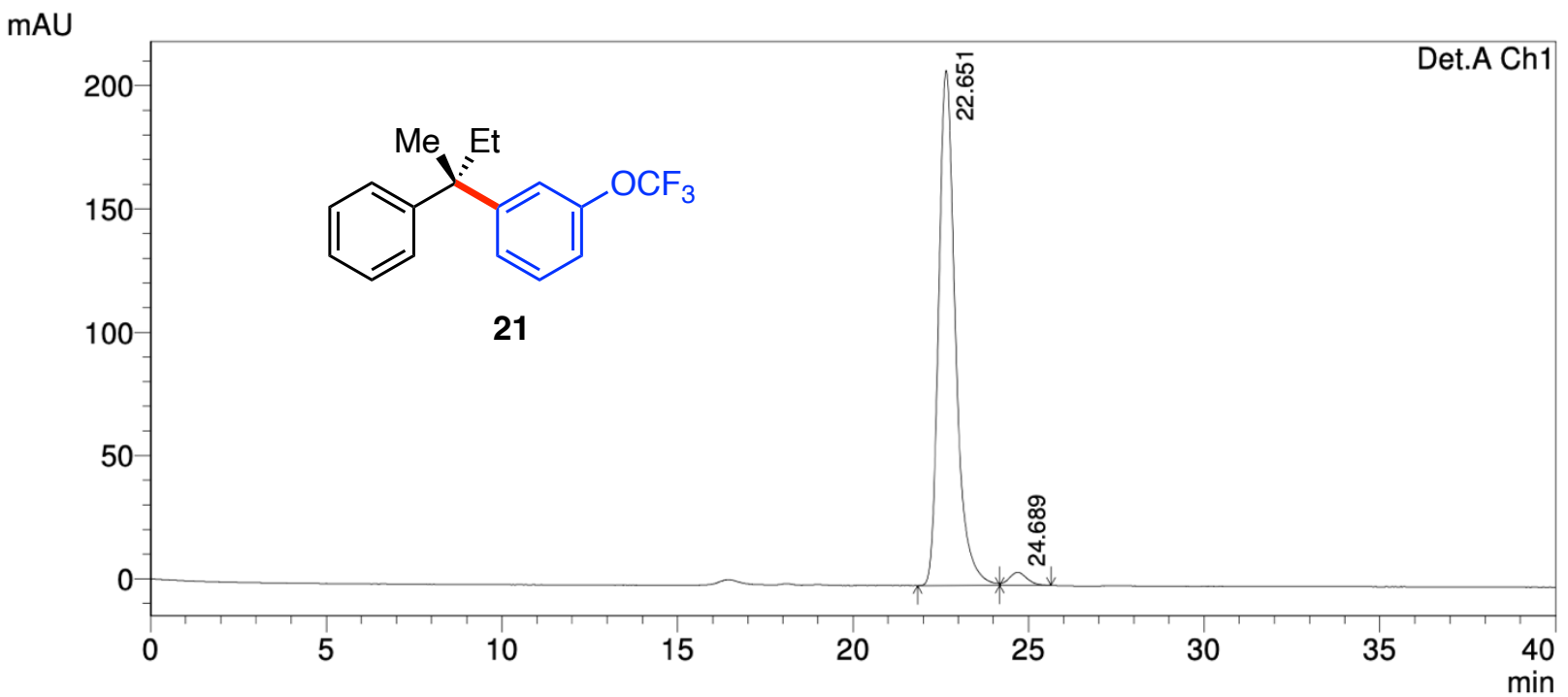

Detector A Ch1 220nm

\begin{tabular}{|r|r|r|r|r|r|}
\hline \multicolumn{1}{|c|}{ Peak\# } & Ret. Time & \multicolumn{1}{|c|}{ Area } & Height & Area \% & \multicolumn{1}{c|}{ Height \% } \\
\hline 1 & 22.651 & 6887925 & 208885 & 97.318 & 97.519 \\
\hline 2 & 24.689 & 189805 & 5314 & 2.682 & 2.481 \\
\hline Total & & 7077730 & 214199 & 100.000 & 100.000 \\
\hline
\end{tabular}




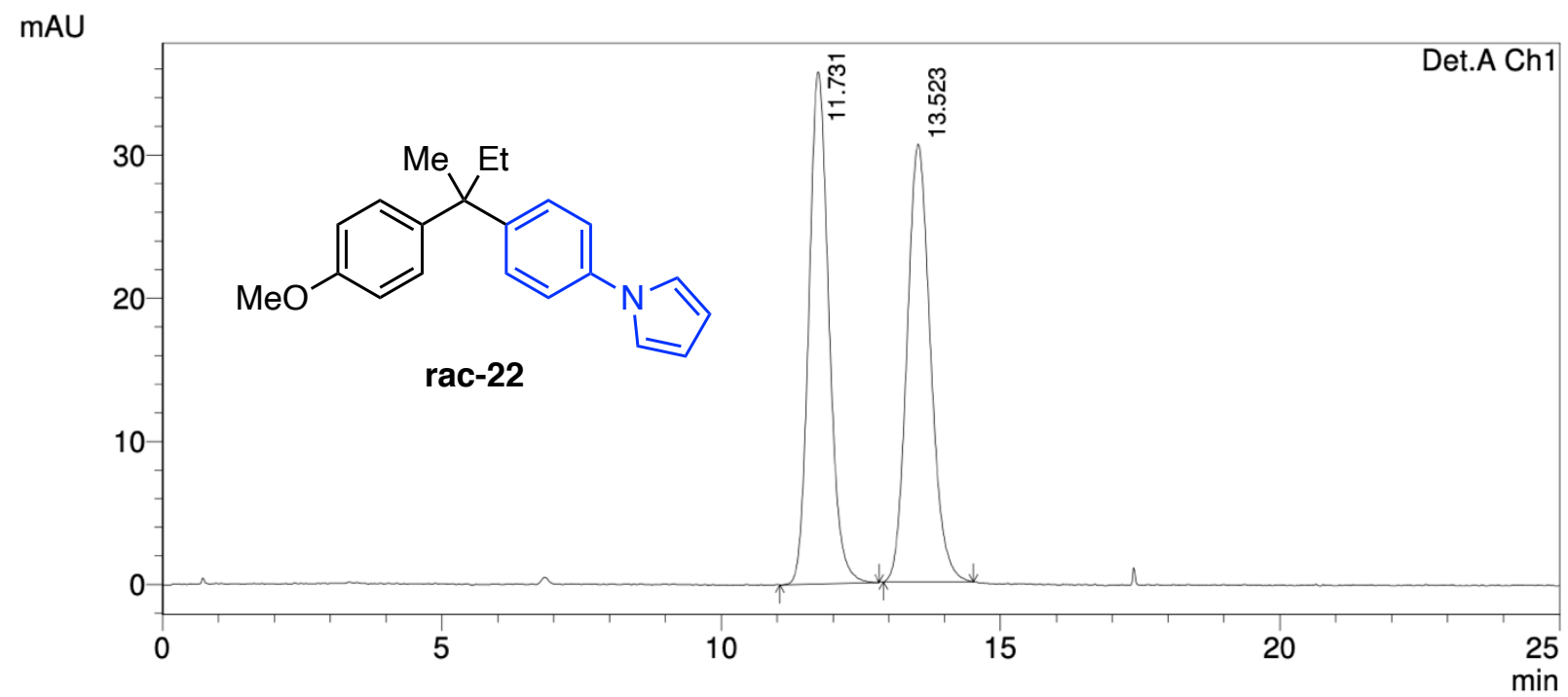

\begin{tabular}{|r|r|r|r|r|r|}
\hline \multicolumn{1}{|c|}{ Peak\# } & Ret. Time & \multicolumn{1}{c|}{ Area } & Height & \multicolumn{1}{|c|}{ Area \% } & \multicolumn{1}{|c|}{ Height \% } \\
\hline 1 & 11.731 & 888643 & 35764 & 49.913 & 53.920 \\
\hline 2 & 13.523 & 891739 & 30563 & 50.087 & 46.080 \\
\hline Total & & 1780381 & 66327 & 100.000 & 100.000 \\
\hline
\end{tabular}

mAU

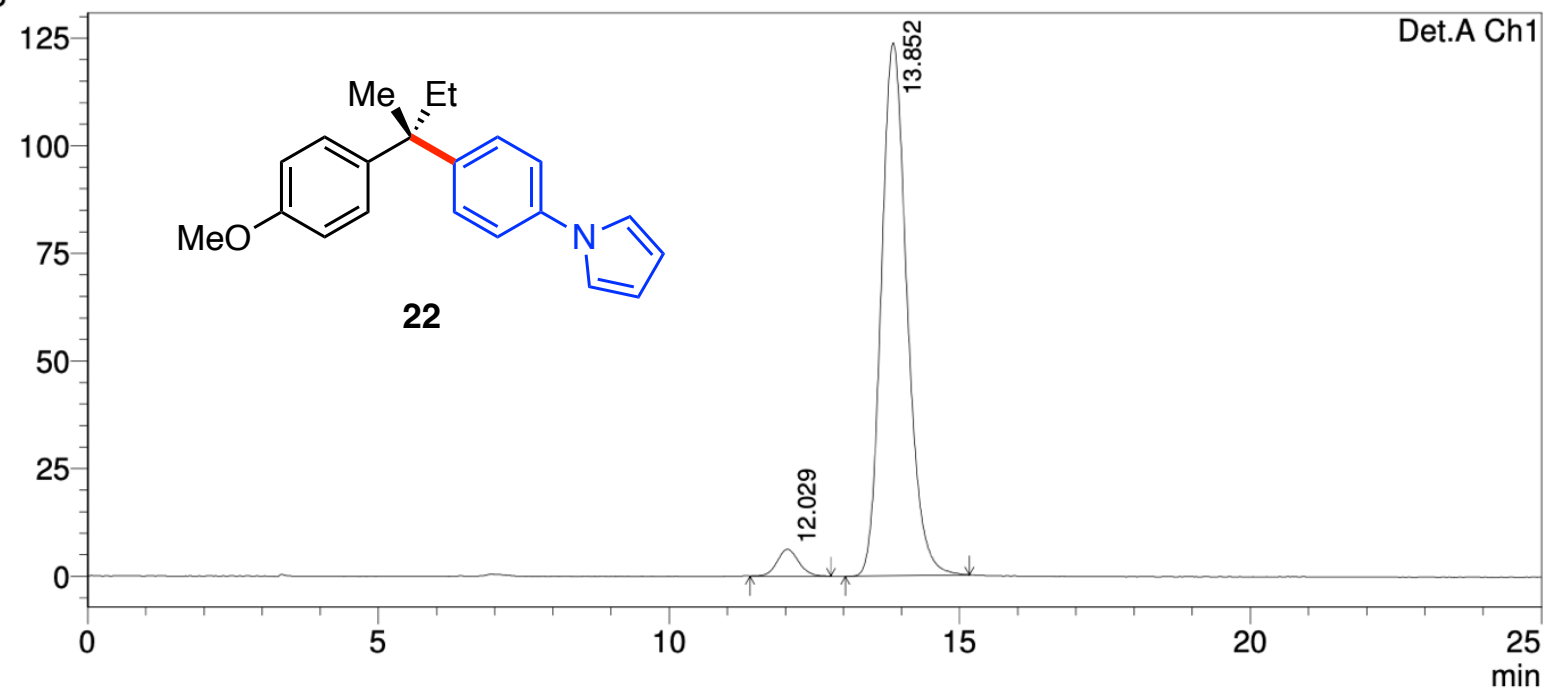

\begin{tabular}{|r|r|r|r|r|r|}
\hline \multicolumn{1}{|c|}{ Peak\# } & Ret. Time & \multicolumn{1}{c|}{ Area } & Height & Area \% & Height \% \\
\hline 1 & 12.029 & 162426 & 6243 & 4.097 & 4.798 \\
\hline 2 & 13.852 & 3802455 & 123879 & 95.903 & 95.202 \\
\hline Total & & 3964882 & 130122 & 100.000 & 100.000 \\
\hline
\end{tabular}




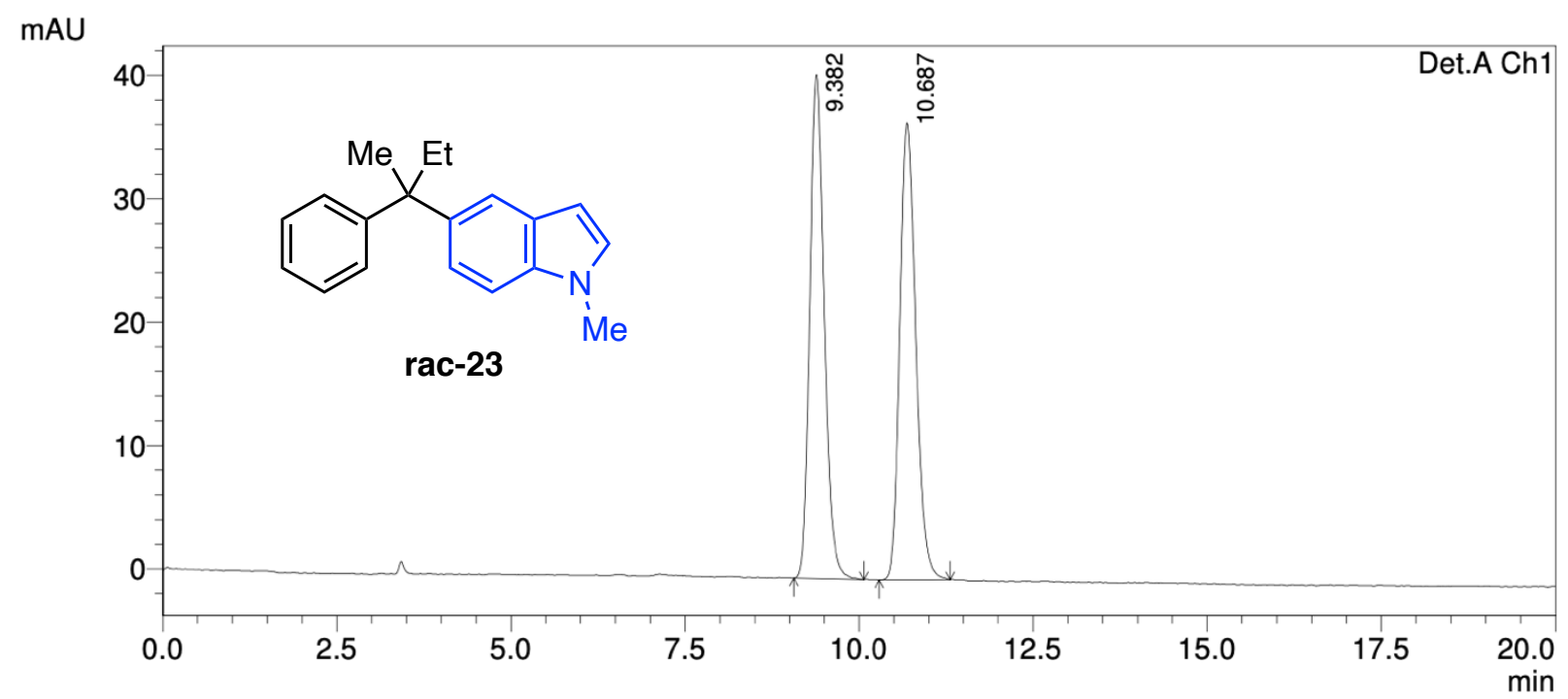

Detector A Ch1 254nm

\begin{tabular}{|r|r|r|r|r|r|}
\hline \multicolumn{1}{|c|}{ Peak\# } & Ret. Time & \multicolumn{1}{|c|}{ Area } & \multicolumn{1}{|c|}{ Height } & Area \% & \multicolumn{1}{|c|}{ Height \% } \\
\hline 1 & 9.382 & 590904 & 40832 & 50.022 & 52.435 \\
\hline 2 & 10.687 & 590393 & 37040 & 49.978 & 47.565 \\
\hline Total & & 1181297 & 77872 & 100.000 & 100.000 \\
\hline
\end{tabular}

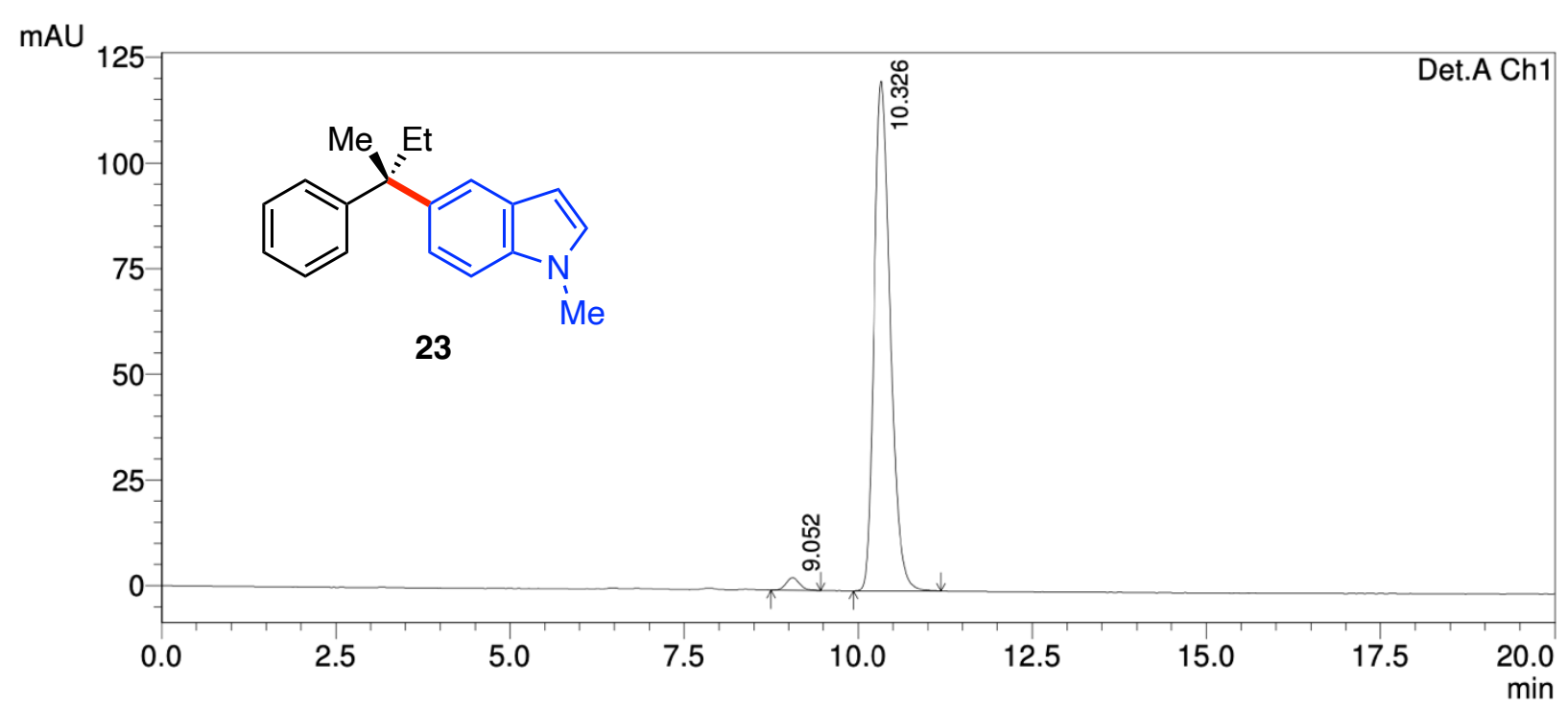

\begin{tabular}{|r|r|r|r|r|r|}
\hline \multicolumn{1}{|c|}{ Peak\# } & Ret. Time & \multicolumn{1}{c|}{ Area } & Height & Area \% & \multicolumn{1}{c|}{ Height \% } \\
\hline 1 & 9.052 & 41142 & 2984 & 2.058 & 2.415 \\
\hline 2 & 10.326 & 1957525 & 120550 & 97.942 & 97.585 \\
\hline Total & & 1998667 & 123533 & 100.000 & 100.000 \\
\hline
\end{tabular}




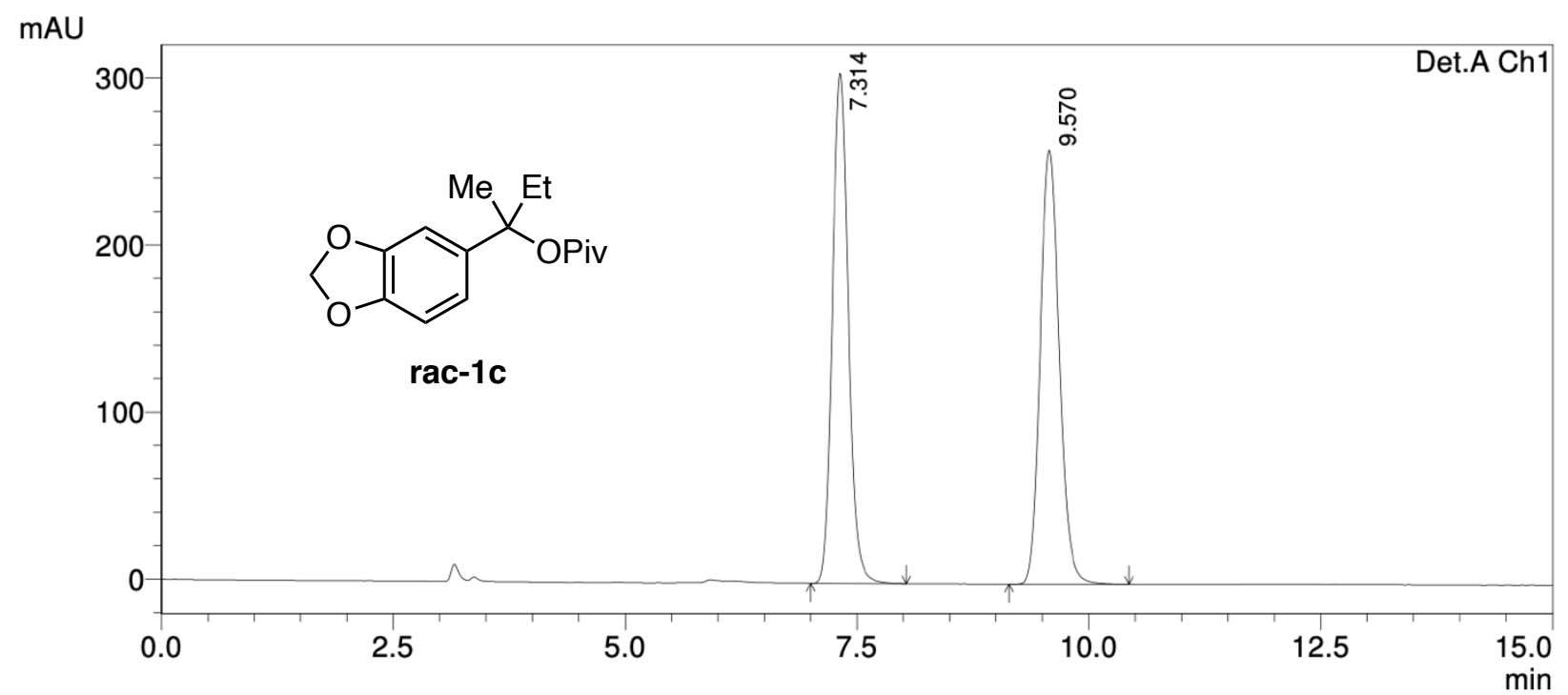

\begin{tabular}{|r|r|r|r|r|r|}
\hline \multicolumn{1}{|c|}{ Peak\# } & Ret. Time & \multicolumn{1}{c|}{ Area } & Height & \multicolumn{1}{c|}{ Area \% } & \multicolumn{1}{c|}{ Height \% } \\
\hline 1 & 7.314 & 3665347 & 305207 & 49.558 & 54.018 \\
\hline 2 & 9.570 & 3730761 & 259804 & 50.442 & 45.982 \\
\hline Total & & 7396108 & 565011 & 100.000 & 100.000 \\
\hline
\end{tabular}

mAU

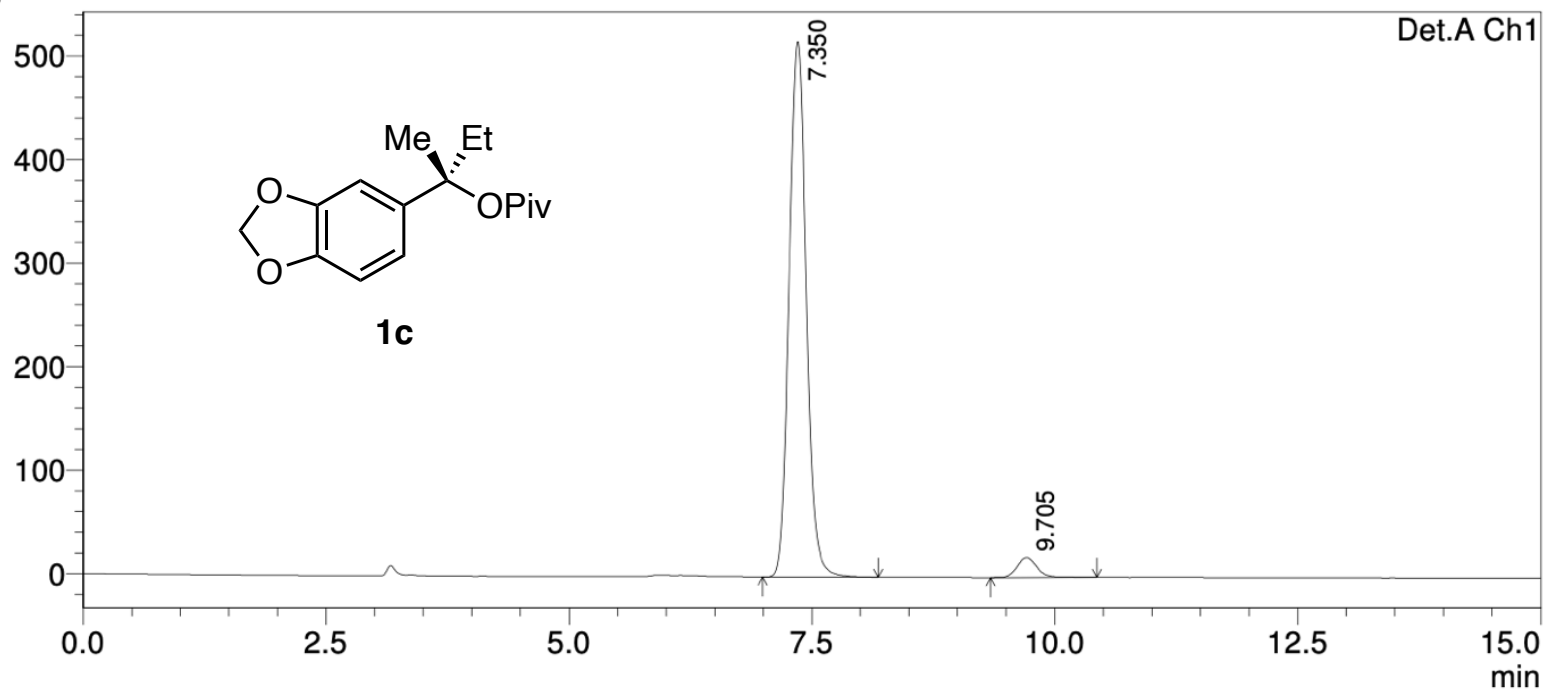

Detector A Ch1 210nm

\begin{tabular}{|r|r|r|r|r|r|}
\hline Peak\# & \multicolumn{1}{|c|}{ Ret. Time } & \multicolumn{1}{|c|}{ Area } & Height & Area \% & \multicolumn{1}{c|}{ Height \% } \\
\hline 1 & 7.350 & 6356991 & 516966 & 95.843 & 96.415 \\
\hline 2 & 9.705 & 275717 & 19222 & 4.157 & 3.585 \\
\hline Total & & 6632708 & 536188 & 100.000 & 100.000 \\
\hline
\end{tabular}




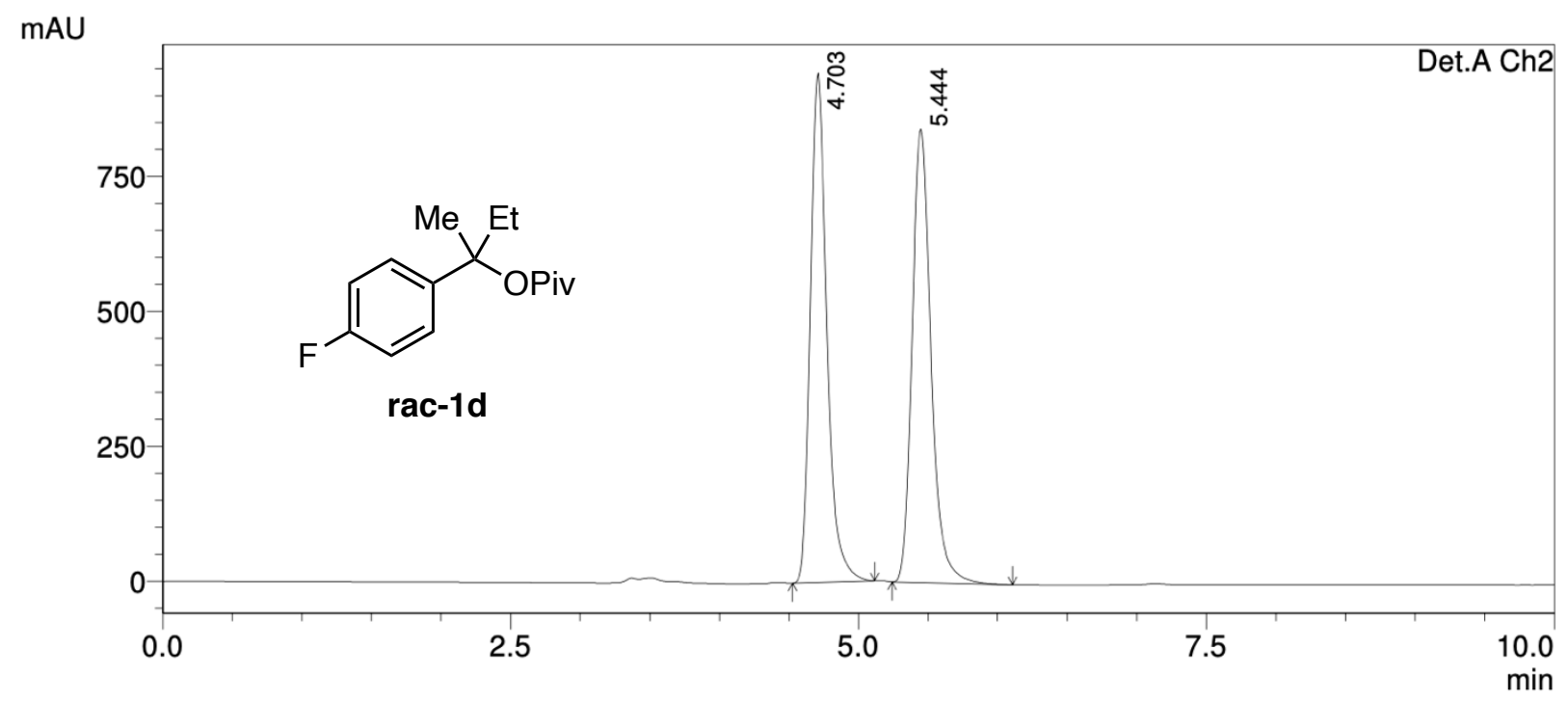

Detector A Ch2 210nm

\begin{tabular}{|r|r|r|r|r|r|}
\hline \multicolumn{1}{|c|}{ Peak\# } & Ret. Time & \multicolumn{1}{|c|}{ Area } & Height & Area \% & \multicolumn{1}{|c|}{ Height \% } \\
\hline 1 & 4.703 & 7787961 & 944461 & 49.526 & 52.893 \\
\hline 2 & 5.444 & 7937063 & 841146 & 50.474 & 47.107 \\
\hline Total & & 15725024 & 1785607 & 100.000 & 100.000 \\
\hline
\end{tabular}

mAU

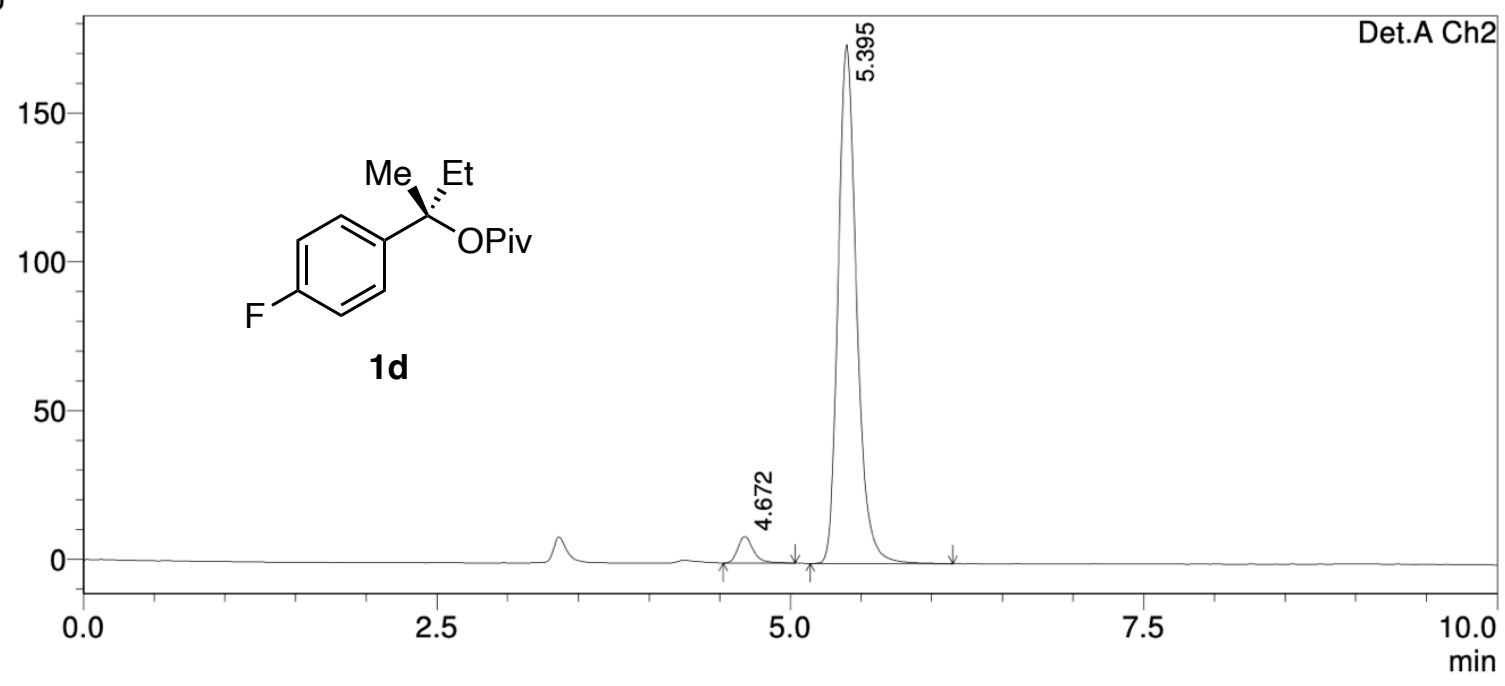

Detector A Ch2 210nm

\begin{tabular}{|r|r|r|r|r|r|}
\hline \multicolumn{1}{|c|}{ Peak\# } & Ret. Time & \multicolumn{1}{c|}{ Area } & Height & Area \% & Height \% \\
\hline 1 & 4.672 & 68298 & 8910 & 4.131 & 4.866 \\
\hline 2 & 5.395 & 1584915 & 174222 & 95.869 & 95.134 \\
\hline Total & & 1653213 & 183132 & 100.000 & 100.000 \\
\hline
\end{tabular}




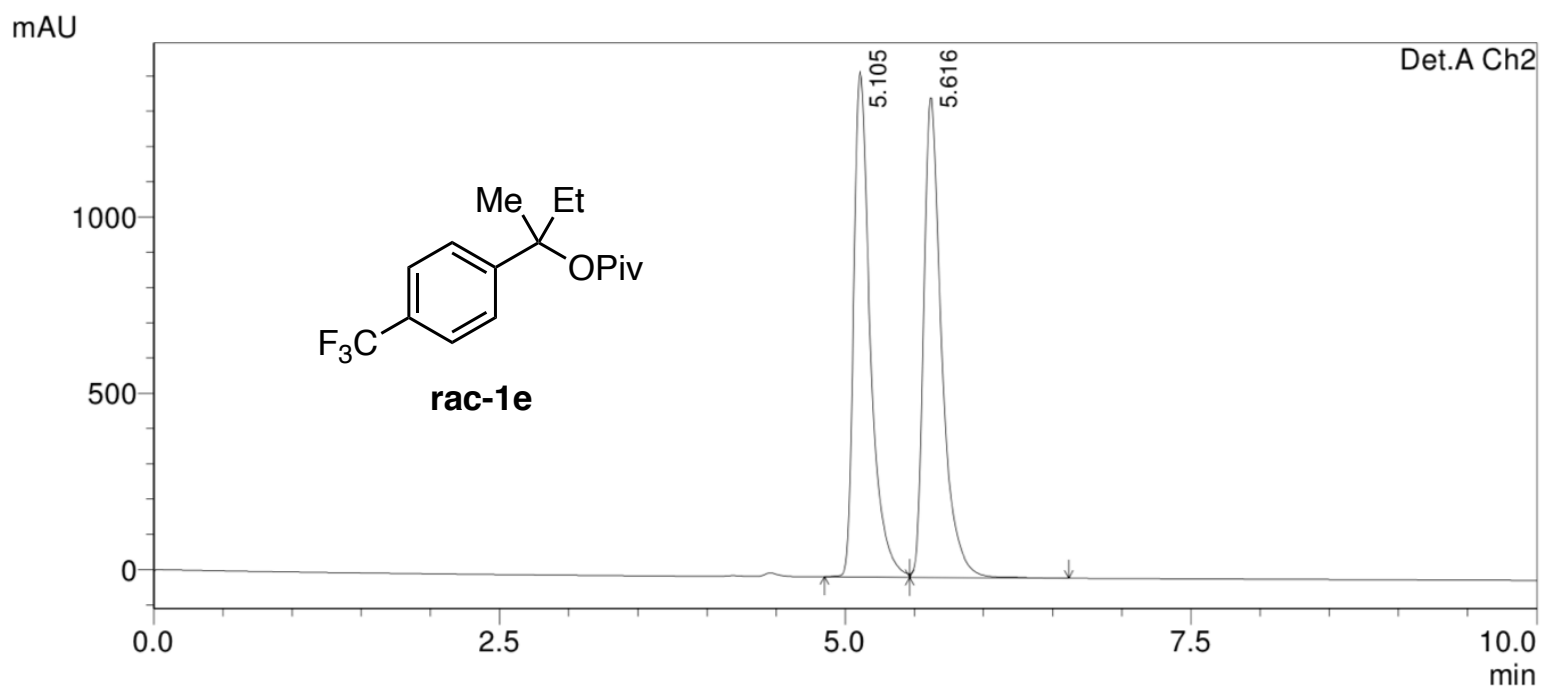

Detector A Ch2 210nm

\begin{tabular}{|r|r|r|r|r|r|}
\hline Peak\# & Ret. Time & Area & \multicolumn{1}{|c|}{ Height } & Area \% & \multicolumn{1}{|c|}{ Height \% } \\
\hline 1 & 5.105 & 12105348 & 1433893 & 49.514 & 51.305 \\
\hline 2 & 5.616 & 12342813 & 1360927 & 50.486 & 48.695 \\
\hline Total & & 24448160 & 2794819 & 100.000 & 100.000 \\
\hline
\end{tabular}

mAU

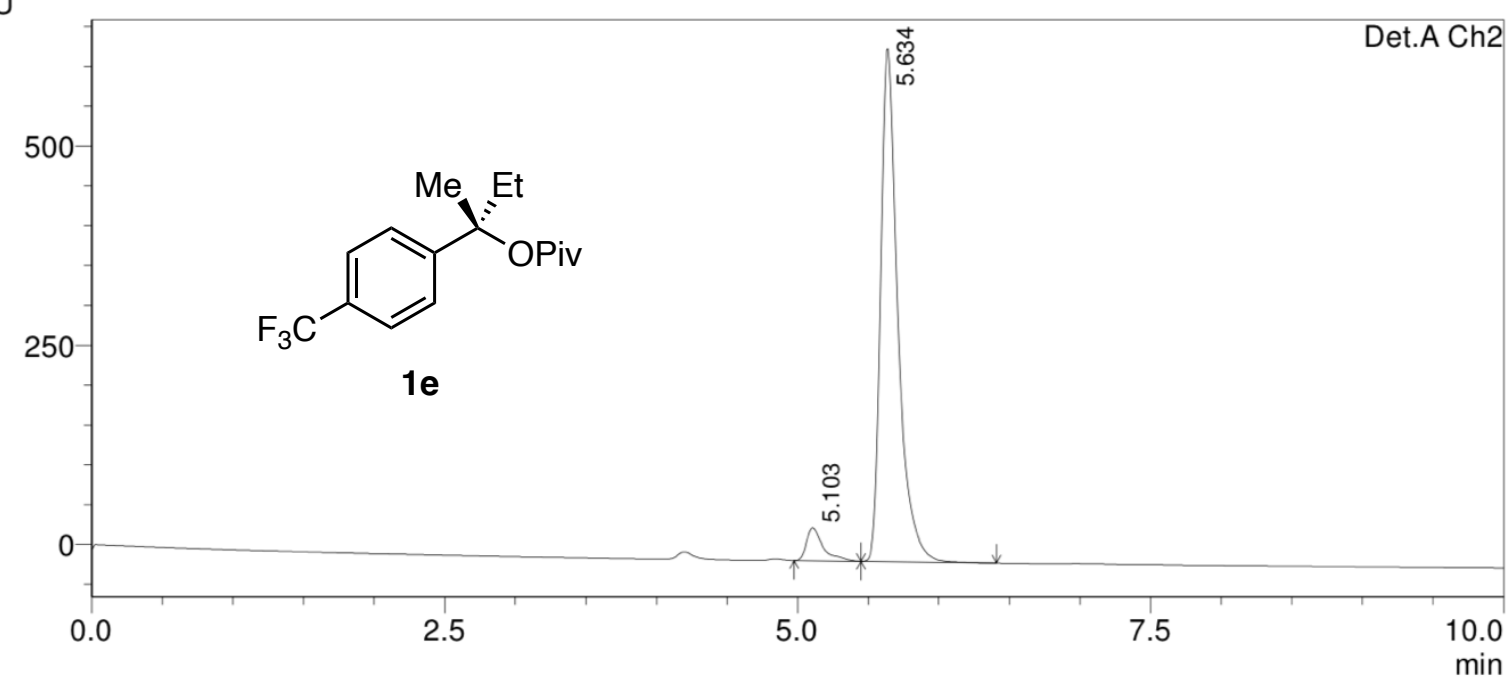

Detector A Ch2 210nm

\begin{tabular}{|r|r|r|r|r|r|}
\hline \multicolumn{1}{|c|}{ Peak\# } & Ret. Time & \multicolumn{1}{|c|}{ Area } & Height & Area \% & \multicolumn{1}{c|}{ Height \% } \\
\hline 1 & 5.103 & 341946 & 41570 & 5.882 & 6.068 \\
\hline 2 & 5.634 & 5471812 & 643454 & 94.118 & 93.932 \\
\hline Total & & 5813758 & 685024 & 100.000 & 100.000 \\
\hline
\end{tabular}




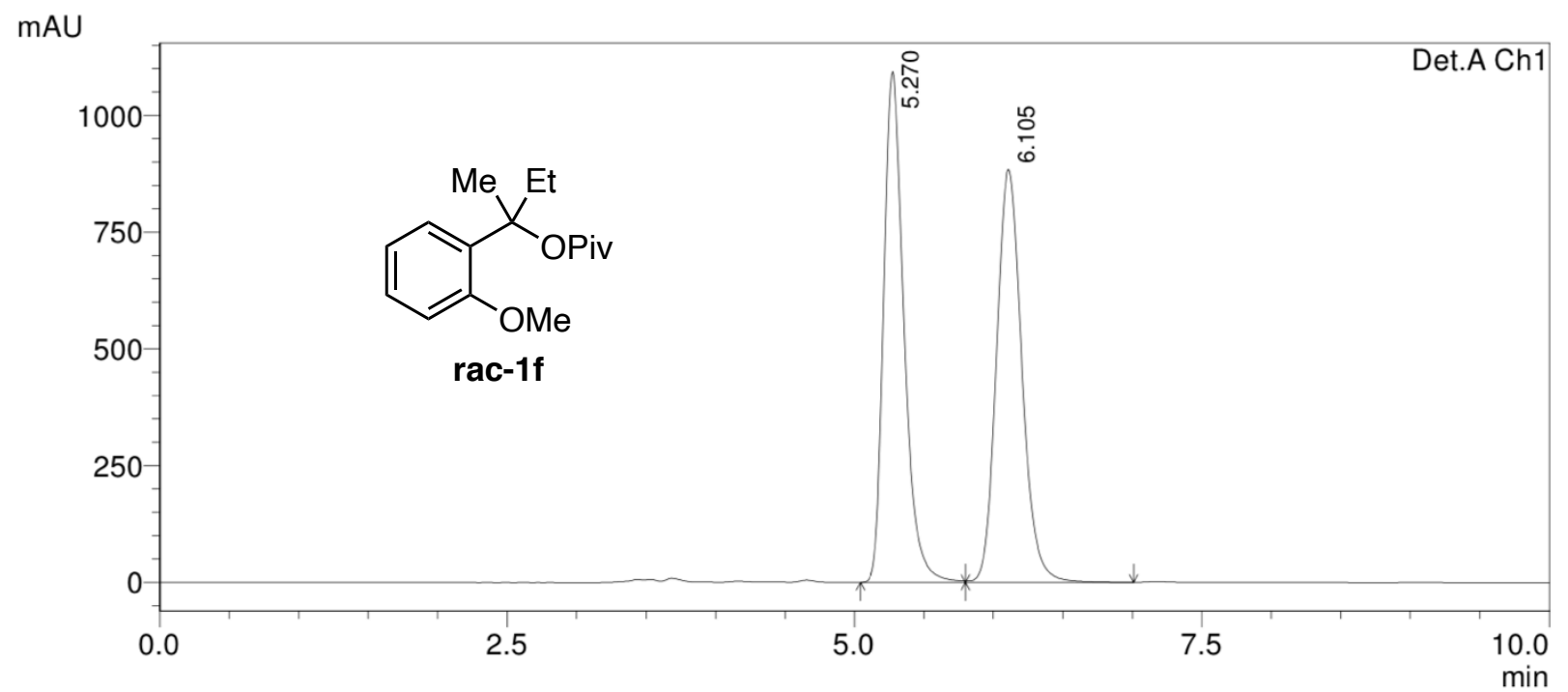

\begin{tabular}{|r|r|r|r|r|r|}
\hline Peak\# & Ret. Time & Area & Height & Area \% & \multicolumn{1}{|c|}{ Height \% } \\
\hline 1 & 5.270 & 11107555 & 1093736 & 49.712 & 55.284 \\
\hline 2 & 6.105 & 11236340 & 884661 & 50.288 & 44.716 \\
\hline Total & & 22343896 & 1978397 & 100.000 & 100.000 \\
\hline
\end{tabular}

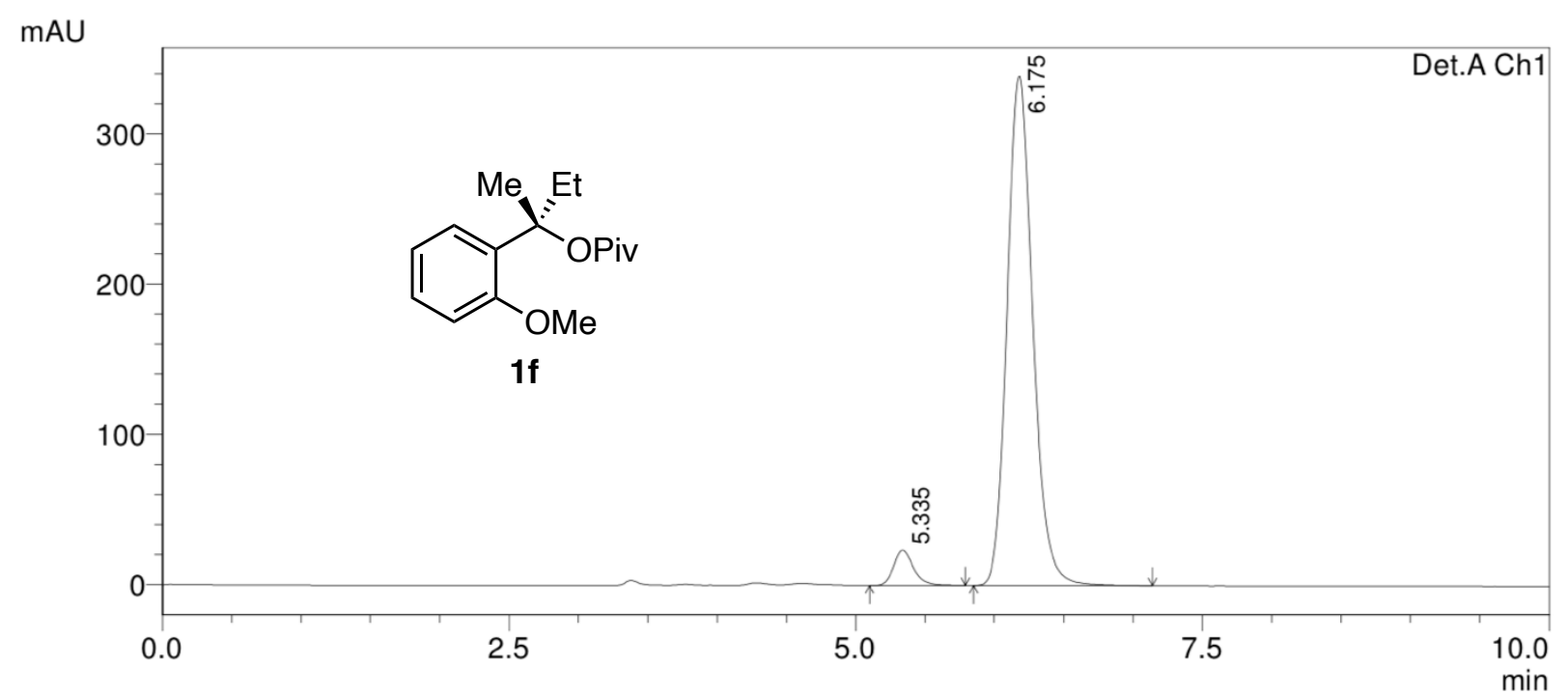

\begin{tabular}{|r|r|r|r|r|r|}
\hline \multicolumn{1}{|c|}{ Peak\# } & Ret. Time & \multicolumn{1}{c|}{ Area } & Height & Area \% & Height \% \\
\hline 1 & 5.335 & 233884 & 23405 & 5.072 & 6.458 \\
\hline 2 & 6.175 & 4377521 & 339010 & 94.928 & 93.542 \\
\hline Total & & 4611405 & 362416 & 100.000 & 100.000 \\
\hline
\end{tabular}




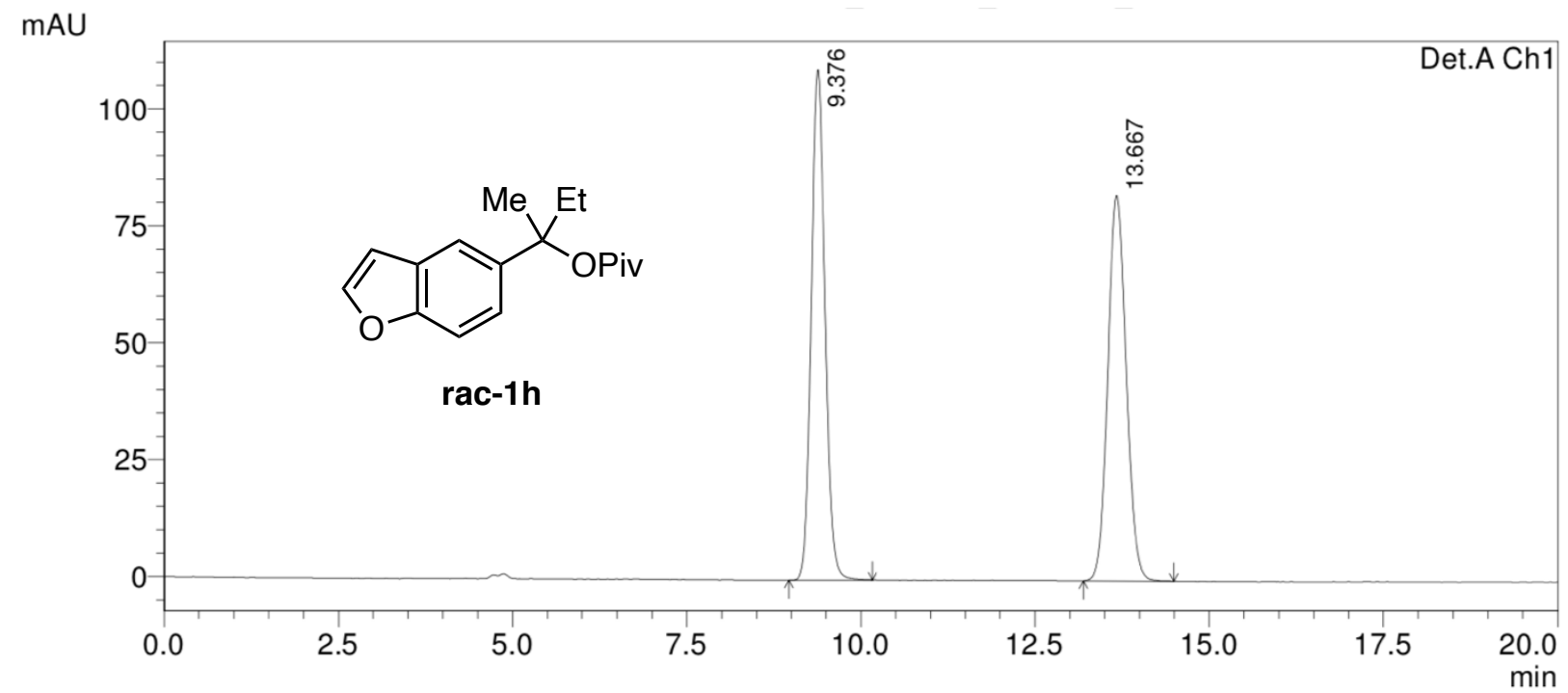

\begin{tabular}{|r|r|r|r|r|r|}
\hline \multicolumn{1}{|c|}{ Peak\# } & Ret. Time & \multicolumn{1}{c|}{ Area } & Height & Area \% & \multicolumn{1}{c|}{ Height \% } \\
\hline 1 & 9.376 & 1500342 & 109097 & 49.778 & 56.966 \\
\hline 2 & 13.667 & 1513701 & 82415 & 50.222 & 43.034 \\
\hline Total & & 3014043 & 191512 & 100.000 & 100.000 \\
\hline
\end{tabular}

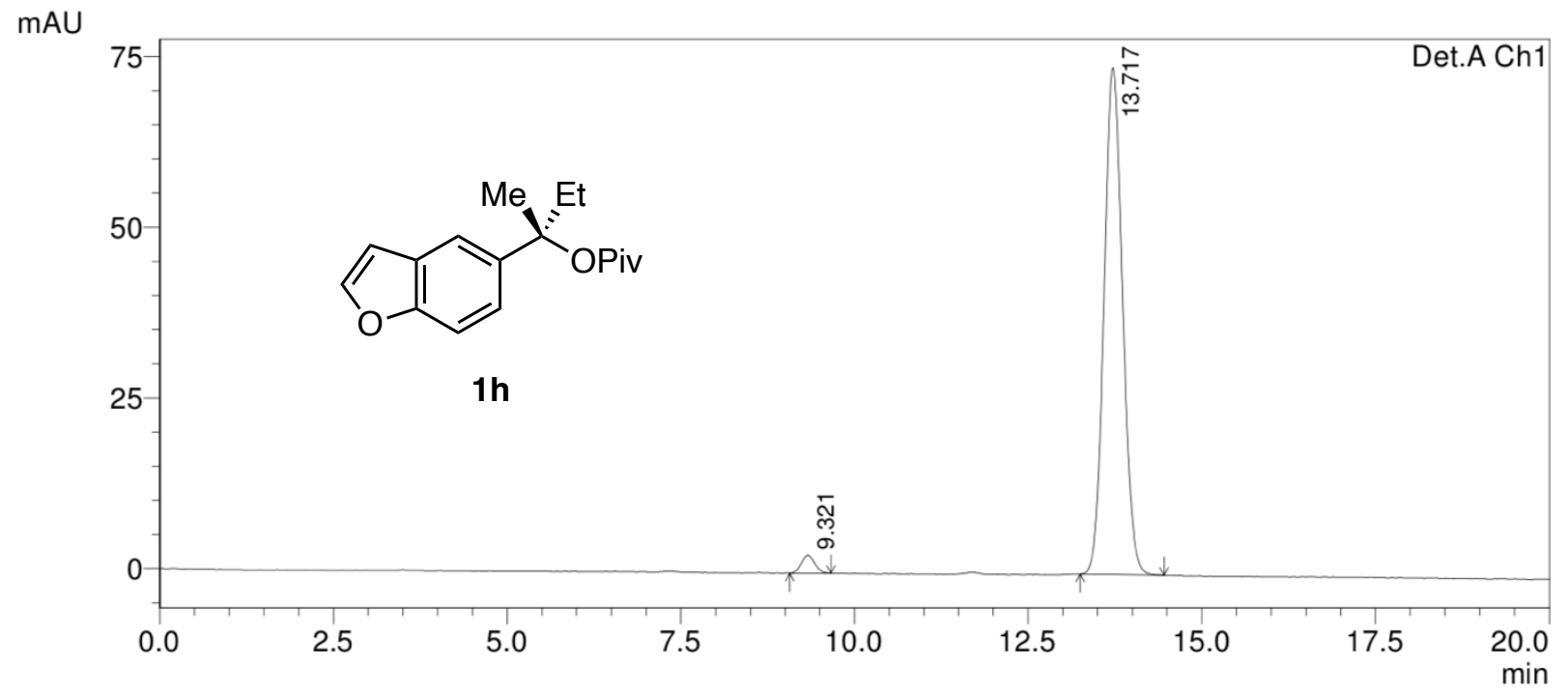

\begin{tabular}{|r|r|r|r|r|r|}
\hline \multicolumn{1}{|c|}{ Peak\# } & Ret. Time & \multicolumn{1}{c|}{ Area } & Height & Area \% & Height \% \\
\hline 1 & 9.321 & 35730 & 2644 & 2.528 & 3.441 \\
\hline 2 & 13.717 & 1377874 & 74197 & 97.472 & 96.559 \\
\hline Total & & 1413604 & 76840 & 100.000 & 100.000 \\
\hline
\end{tabular}




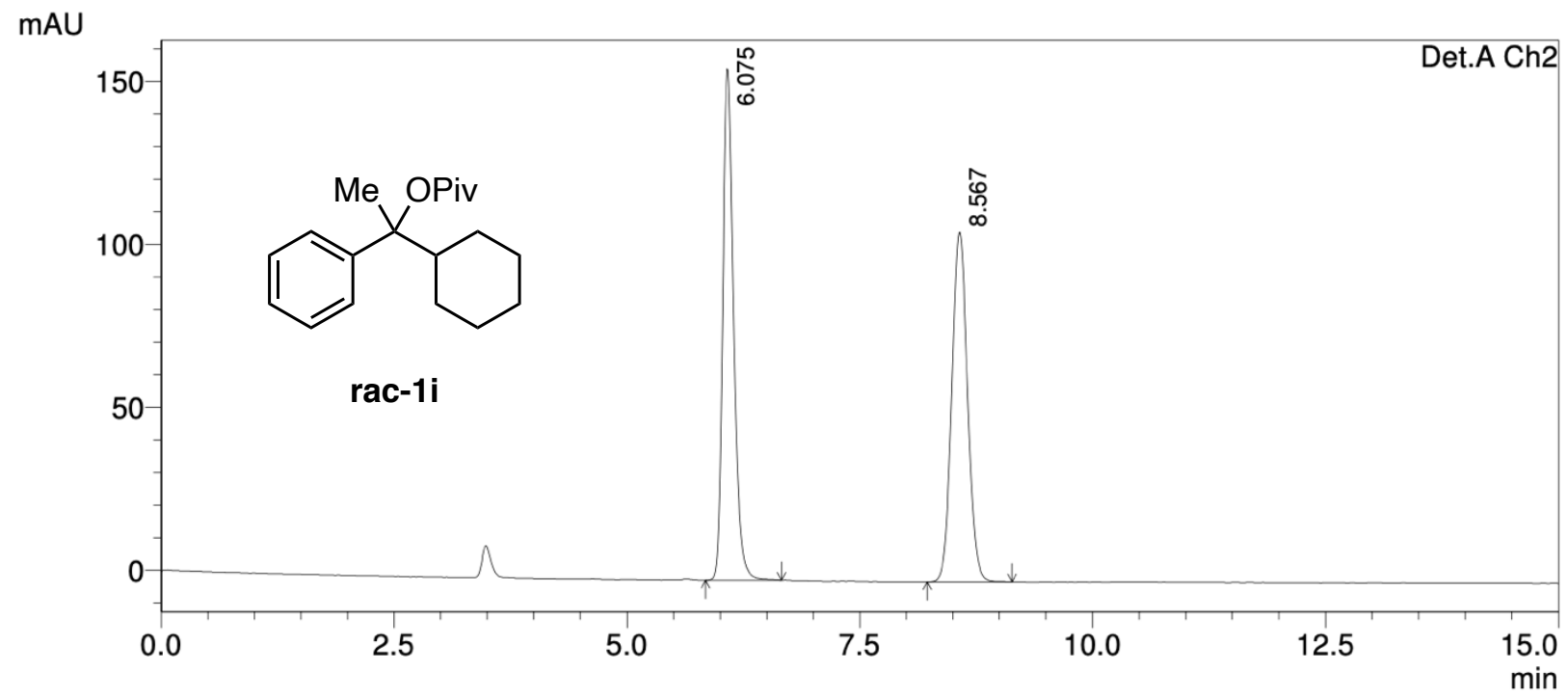

Detector A Ch2 210nm

\begin{tabular}{|r|r|r|r|r|r|}
\hline \multicolumn{1}{|c|}{ Peak\# } & Ret. Time & \multicolumn{1}{|c|}{ Area } & Height & Area \% & \multicolumn{1}{c|}{ Height \% } \\
\hline 1 & 6.075 & 1278597 & 156883 & 50.291 & 59.378 \\
\hline 2 & 8.567 & 1263815 & 107326 & 49.709 & 40.622 \\
\hline Total & & 2542412 & 264210 & 100.000 & 100.000 \\
\hline
\end{tabular}

mAU

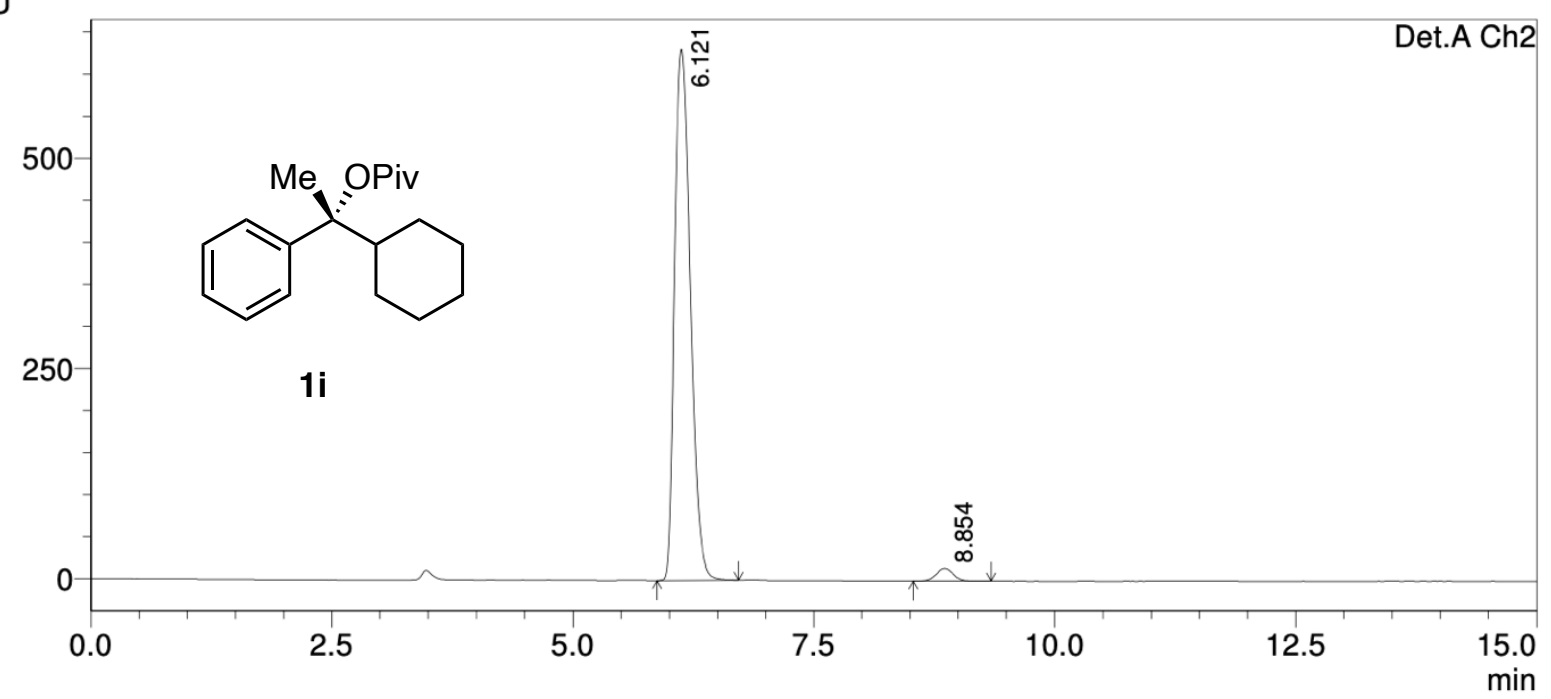

Detector A Ch2 210nm

\begin{tabular}{|r|r|r|r|r|r|}
\hline Peak\# & Ret. Time & \multicolumn{1}{c|}{ Area } & Height & Area \% & \multicolumn{1}{c|}{ Height \% } \\
\hline 1 & 6.121 & 7231946 & 631871 & 97.506 & 97.669 \\
\hline 2 & 8.854 & 184968 & 15083 & 2.494 & 2.331 \\
\hline Total & & 7416913 & 646954 & 100.000 & 100.000 \\
\hline
\end{tabular}




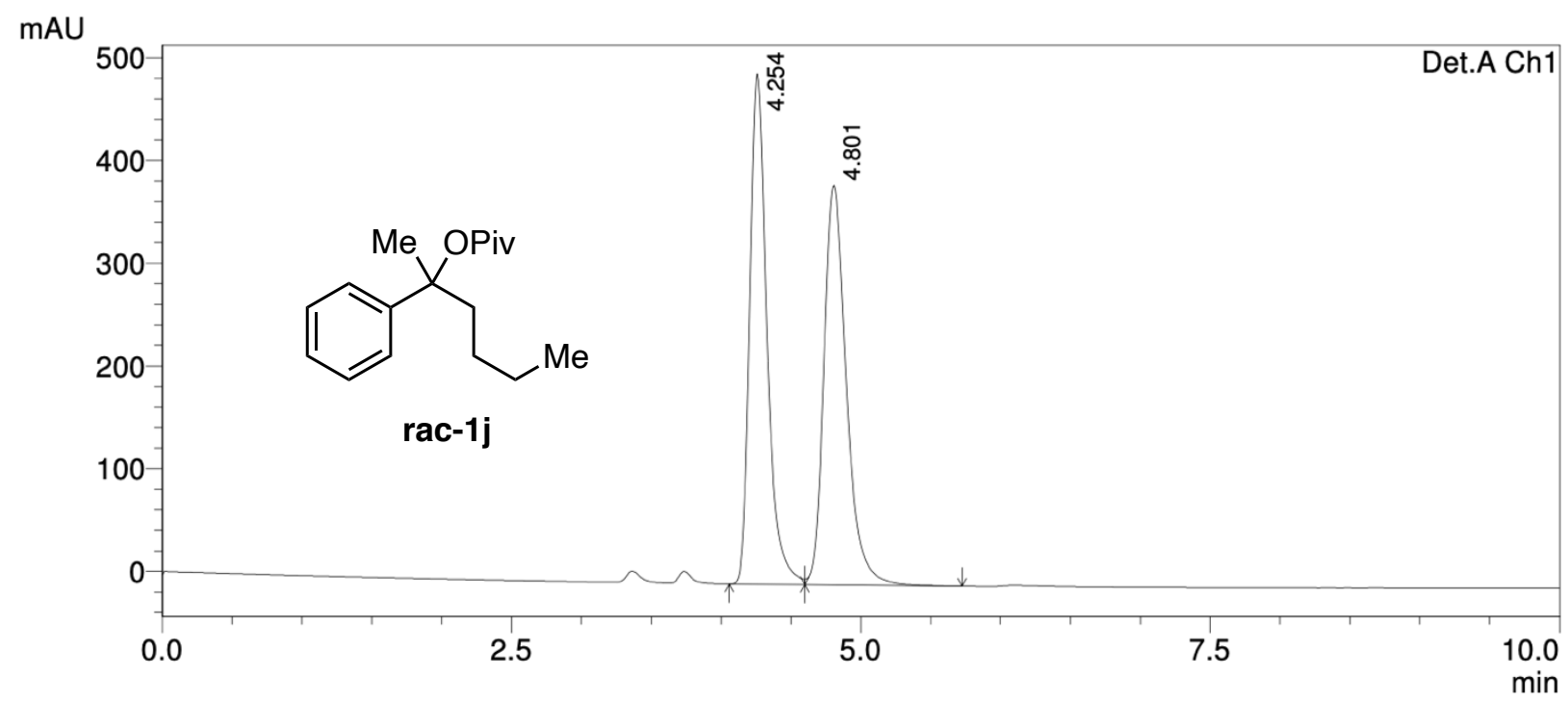

Detector A Ch1 210nm

\begin{tabular}{|r|r|r|r|r|r|}
\hline Peak\# & Ret. Time & Area & Height & Area \% & \multicolumn{1}{|c|}{ Height \% } \\
\hline 1 & 4.254 & 4263843 & 496736 & 49.502 & 56.096 \\
\hline 2 & 4.801 & 4349654 & 388778 & 50.498 & 43.904 \\
\hline Total & & 8613497 & 885514 & 100.000 & 100.000 \\
\hline
\end{tabular}

mAU

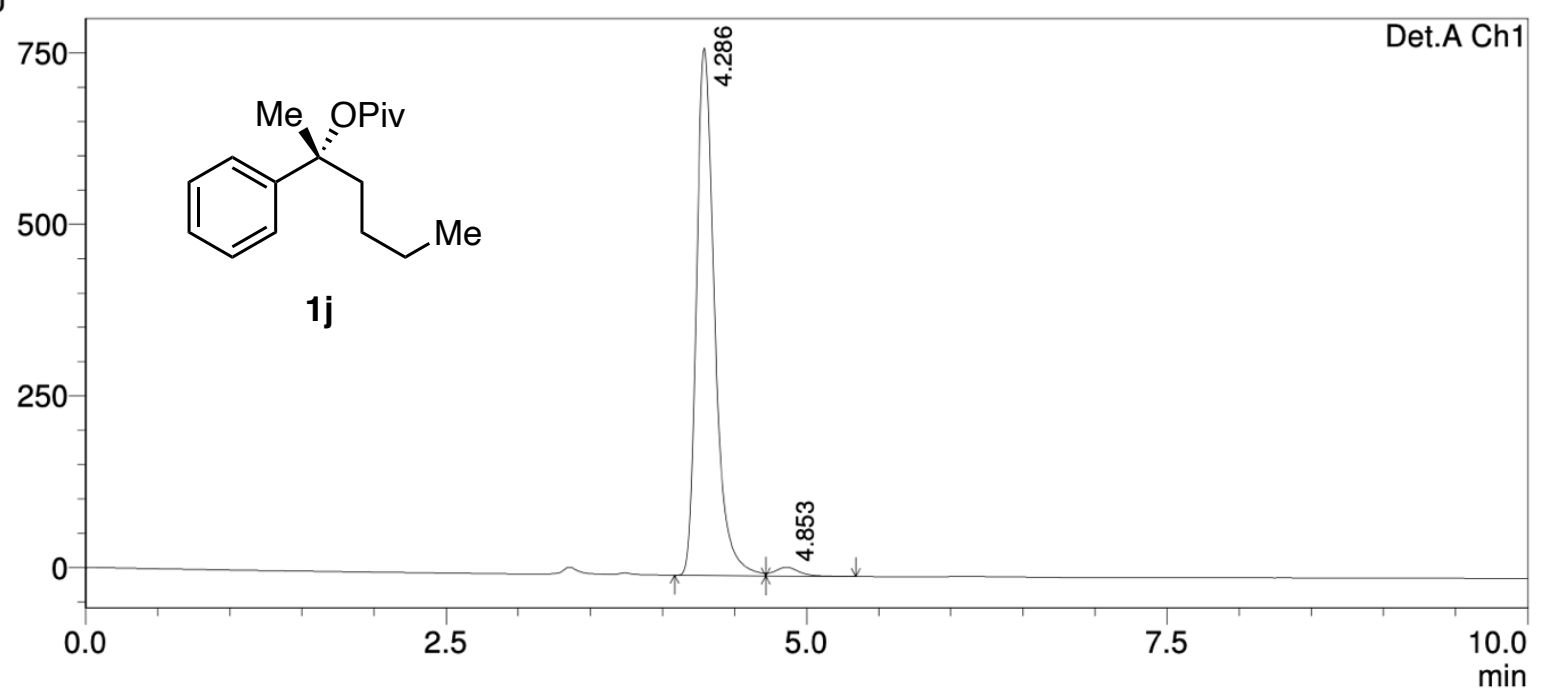

\begin{tabular}{|r|r|r|r|r|r|}
\hline \multicolumn{1}{|c|}{ Peak\# } & Ret. Time & \multicolumn{1}{c|}{ Area } & Height & Area \% & Height \% \\
\hline 1 & 4.286 & 6731255 & 768399 & 97.863 & 98.382 \\
\hline 2 & 4.853 & 146958 & 12635 & 2.137 & 1.618 \\
\hline Total & & 6878213 & 781034 & 100.000 & 100.000 \\
\hline
\end{tabular}




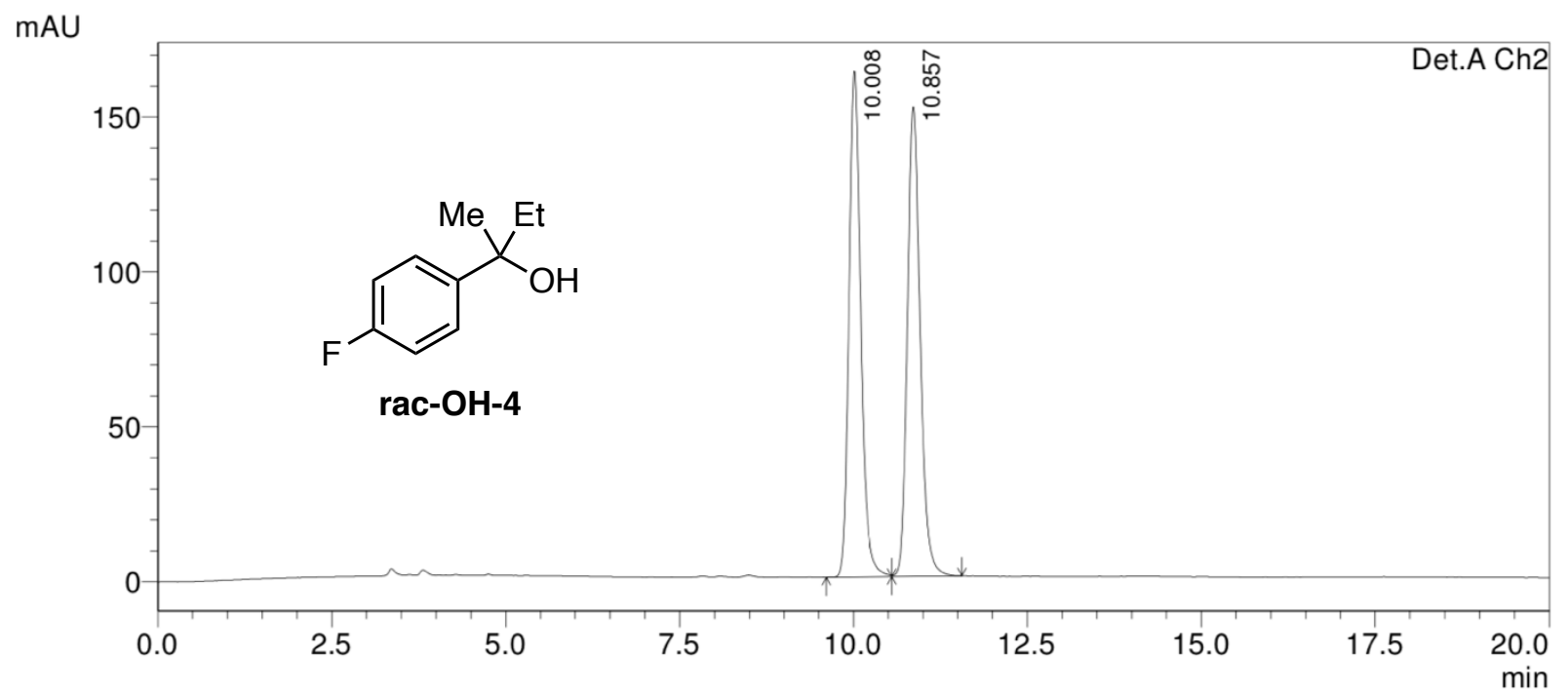

Detector A Ch2 210nm

\begin{tabular}{|r|r|r|r|r|r|}
\hline \multicolumn{1}{|c|}{ Peak\# } & Ret. Time & Area & Height & Area \% & \multicolumn{1}{|c|}{ Height \% } \\
\hline 1 & 10.008 & 1939776 & 163289 & 49.982 & 51.856 \\
\hline 2 & 10.857 & 1941164 & 151601 & 50.018 & 48.144 \\
\hline Total & & 3880939 & 314890 & 100.000 & 100.000 \\
\hline
\end{tabular}

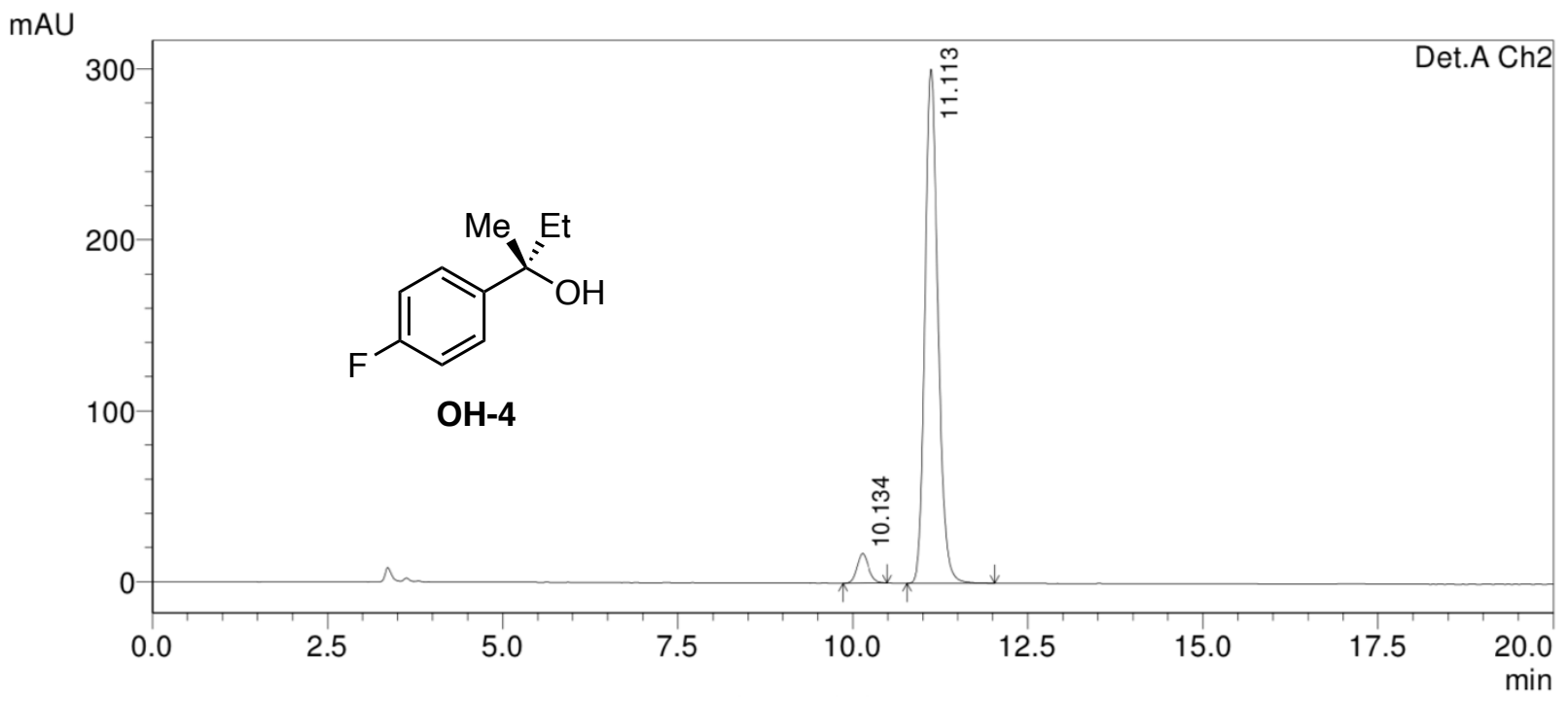

Detector A Ch2 210nm

\begin{tabular}{|r|r|r|r|r|r|}
\hline \multicolumn{1}{|c|}{ Peak\# } & Ret. Time & \multicolumn{1}{c|}{ Area } & Height & Area \% & \multicolumn{1}{|c|}{ Height \% } \\
\hline 1 & 10.134 & 201296 & 17310 & 4.886 & 5.441 \\
\hline 2 & 11.113 & 3918254 & 300844 & 95.114 & 94.559 \\
\hline Total & & 4119550 & 318154 & 100.000 & 100.000 \\
\hline
\end{tabular}




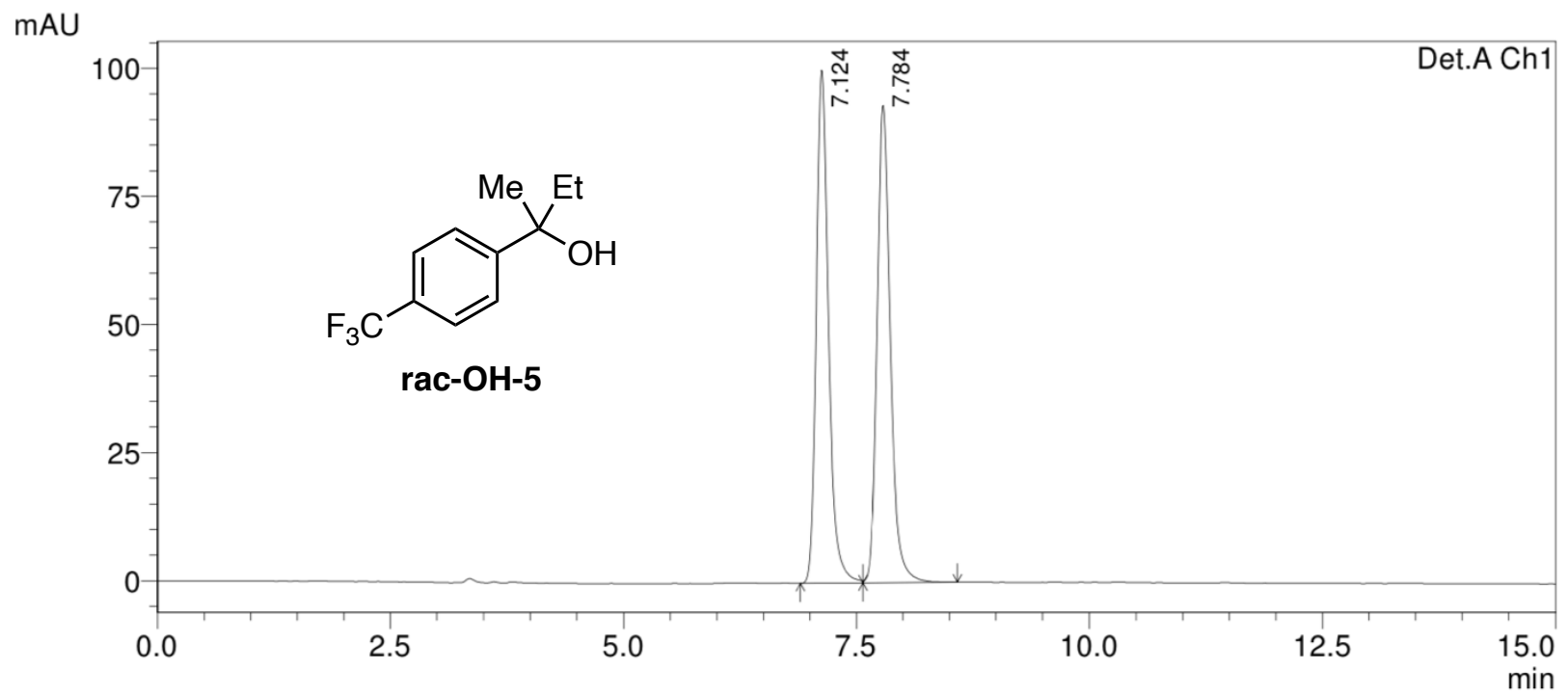

Detector A Ch1 220nm

\begin{tabular}{|r|r|r|r|r|r|}
\hline \multicolumn{1}{|c|}{ Peak\# } & Ret. Time & \multicolumn{1}{c|}{ Area } & Height & \multicolumn{1}{c|}{ Area \% } & \multicolumn{1}{c|}{ Height \% } \\
\hline 1 & 7.124 & 919433 & 100125 & 49.798 & 51.804 \\
\hline 2 & 7.784 & 926894 & 93151 & 50.202 & 48.196 \\
\hline Total & & 1846327 & 193277 & 100.000 & 100.000 \\
\hline
\end{tabular}

mAU

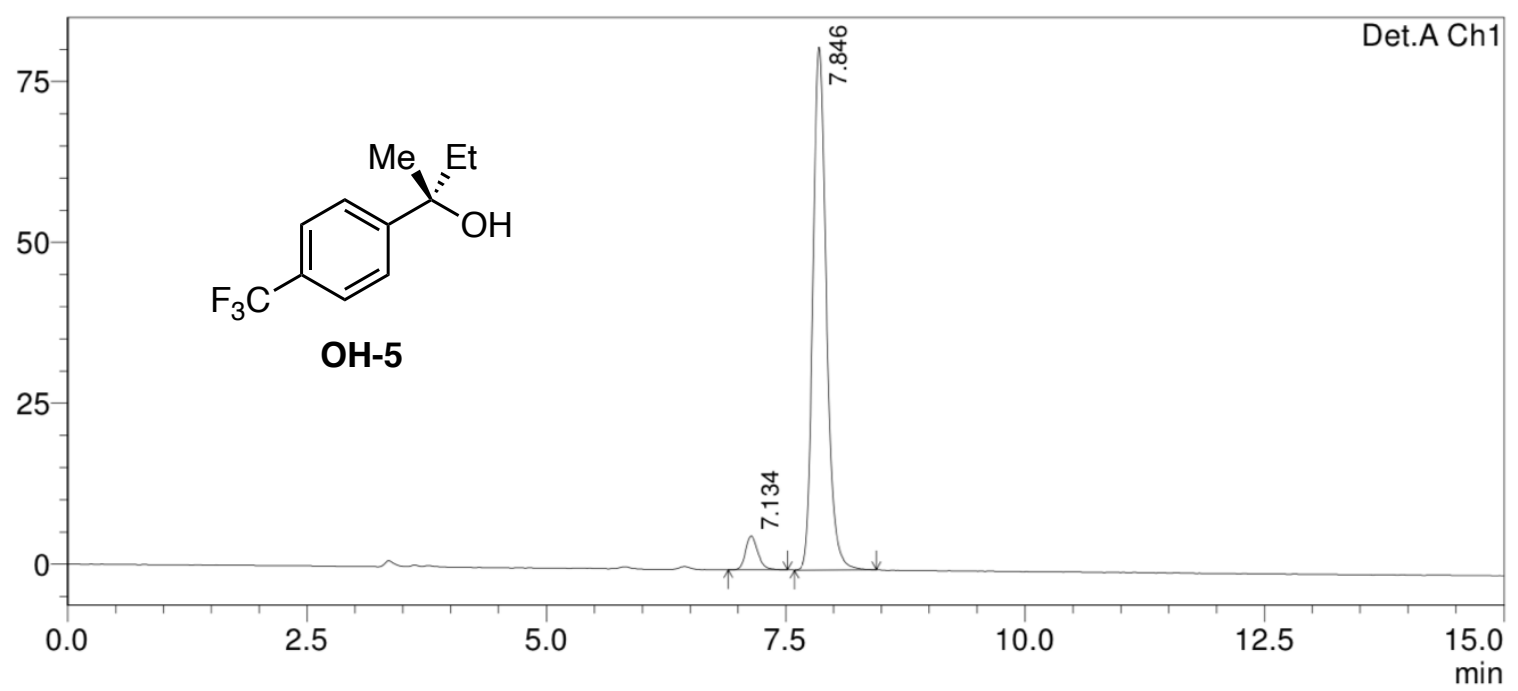

Detector A Ch1 220nm

\begin{tabular}{|r|r|r|r|r|r|}
\hline \multicolumn{1}{|c|}{ Peak\# } & Ret. Time & \multicolumn{1}{|c|}{ Area } & Height & Area \% & Height \% \\
\hline 1 & 7.134 & 47541 & 5256 & 5.619 & 6.076 \\
\hline 2 & 7.846 & 798550 & 81253 & 94.381 & 93.924 \\
\hline Total & & 846091 & 86509 & 100.000 & 100.000 \\
\hline
\end{tabular}




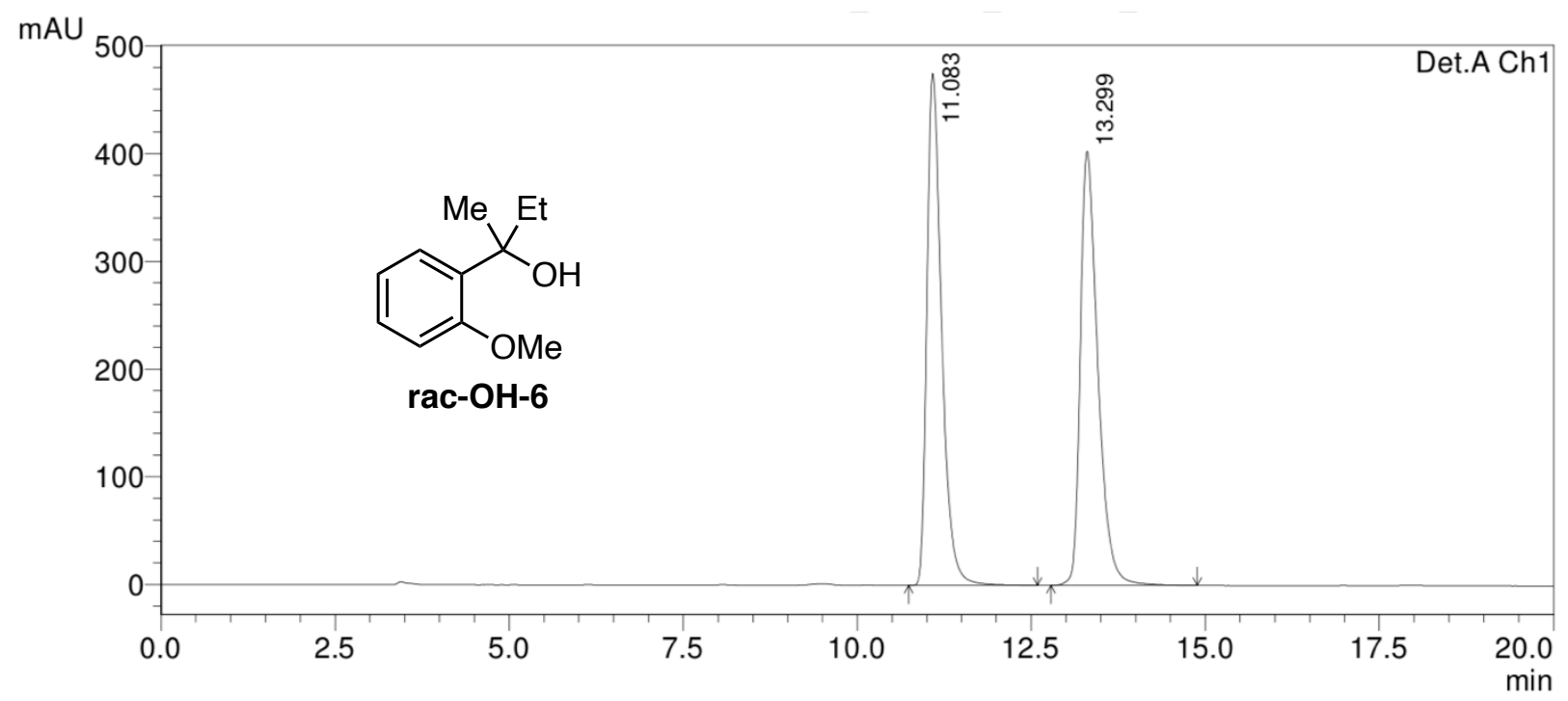

Detector A Ch1 220nm

\begin{tabular}{|r|r|r|r|r|r|}
\hline \multicolumn{1}{|c|}{ Peak\# } & Ret. Time & \multicolumn{1}{c|}{ Area } & Height & Area \% & Height \% \\
\hline 1 & 11.083 & 6994641 & 475042 & 49.737 & 54.073 \\
\hline 2 & 13.299 & 7068513 & 403477 & 50.263 & 45.927 \\
\hline Total & & 14063154 & 878520 & 100.000 & 100.000 \\
\hline
\end{tabular}

mAU

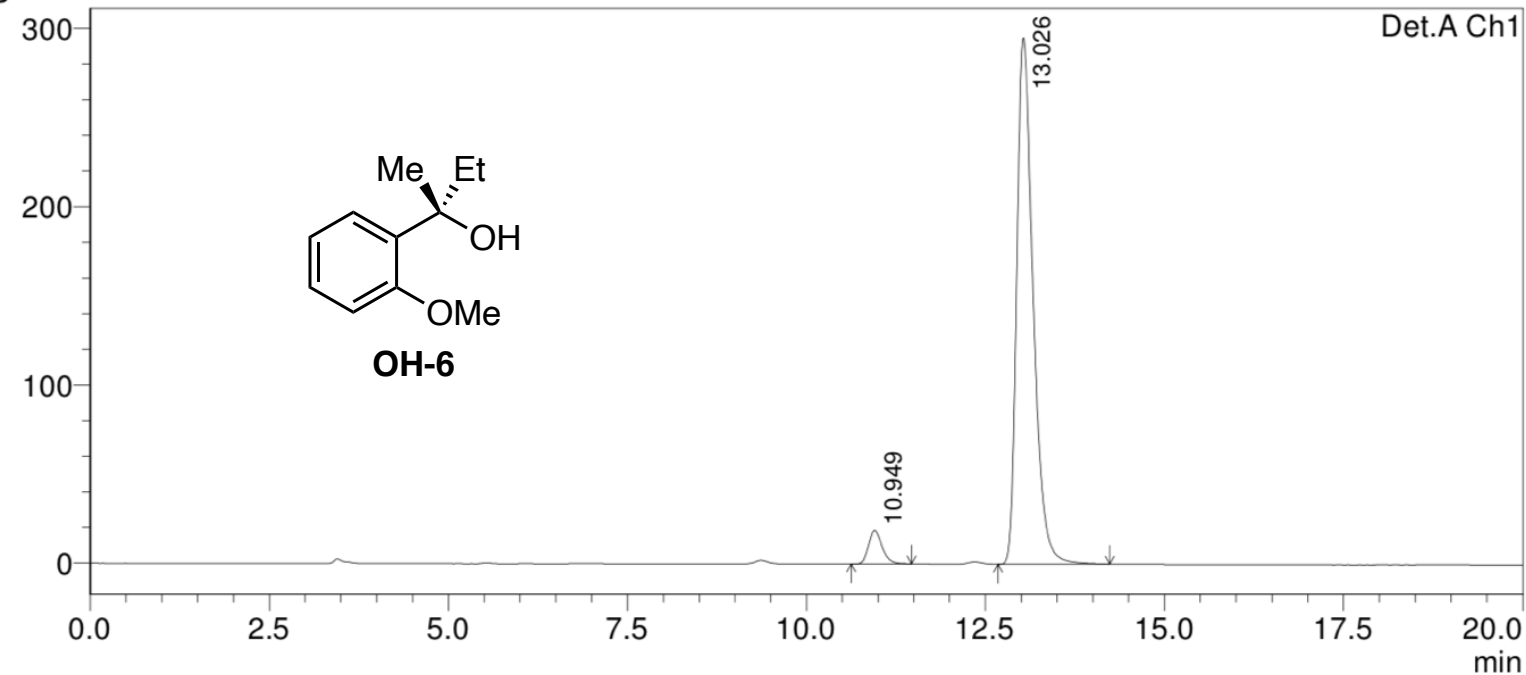

Detector A Ch1 220nm

\begin{tabular}{|r|r|r|r|r|r|}
\hline \multicolumn{1}{|c|}{ Peak\# } & Ret. Time & \multicolumn{1}{c|}{ Area } & Height & Area \% & Height \% \\
\hline 1 & 10.949 & 249710 & 18747 & 5.057 & 5.970 \\
\hline 2 & 13.026 & 4688564 & 295258 & 94.943 & 94.030 \\
\hline Total & & 4938273 & 314005 & 100.000 & 100.000 \\
\hline
\end{tabular}

